\title{
The Wall Pressure Spectrum of High Reynolds Number Rough-Wall Turbulent Boundary Layers
}

\author{
Jonathan Bradley Forest
}

Thesis submitted to the faculty of the Virginia Polytechnic Institute and State University in partial fulfillment of the requirements for the degree of

Master of Science
in
Aerospace Engineering

\author{
William J. Devenport \\ Kevin T. Lowe \\ Joseph A. Schetz \\ Jason M. Anderson
}

$01 / 19 / 2012$

Blacksburg, Virginia

Keywords: High Reynolds number, rough wall, turbulent boundary layer, surface pressure 


\title{
The Wall Pressure Spectrum of High Reynolds Number Rough- Wall Turbulent Boundary Layers
}

\author{
Jonathan Bradley Forest
}

\begin{abstract}
The presence of roughness on a surface subject to high Reynolds number flows promotes the formation of a turbulent boundary layer and the generation of a fluctuating pressure field imposed on the surface. While numerous studies have investigated the wall pressure fluctuations over zero-pressure gradient smooth walls, few studies have examined the effects of surface roughness on the wall pressure field. Additionally, due to the difficulties in obtaining high Reynolds number flows over fully rough surfaces in laboratory settings, an even fewer number of studies have investigated this phenomenon under flow conditions predicted to be fully free of transitional effects that would ensure similarity laws could be observed. This study presents the efforts to scale and describe the wall pressure spectrum of a rough wall, high Reynolds number turbulent boundary layer free of transitional effects. Measurements were taken in the Virginia Tech Stability Wind Tunnel for both smooth and rough walls. A deterministic roughness fetch composed of 3$\mathrm{mm}$ hemispheres arranged in a $16.5-\mathrm{mm}$ square array was used for the rough surface. Smooth and rough wall flows were examined achieving Reynolds numbers up to $R_{\theta}=$ 68700 and $R e_{\theta}=80200$ respectively, with the rough wall flows reaching roughness based Reynolds numbers up to $k_{g}{ }^{+}=507$ with a simultaneous blockage ratio of $\delta / k_{g}=76$. A new roughness based inner variable scaling is proposed that provides a much more complete collapse of the rough wall pressure spectra than previous scales had provided over a large range of Reynolds numbers and roughness configurations. This scaling implies the presence of two separate time scales associated with the near wall turbulence structure generation. A clearly defined overlap region was observed for the rough wall surface pressure spectra displaying a frequency dependence of $\omega^{-1.33}$, believed to be a function of the surface roughness configuration and its associated transport of turbulent energy. The rough wall pressure spectra were shown to decay more rapidly, but based on the same function as what defined the smooth wall decay.
\end{abstract}




\section{Acknowledgements}

I would like to start off by thanking my family. Without their support, I would not have had the opportunities that I have had. Their support and encouragement helped guide me through some of the more stressful periods of my education.

I owe a great deal of gratitude to my advisor Dr. William Devenport. His knowledge and guidance have helped direct me throughout my graduate and research career, while his enthusiasm has helped propel me to a larger depth of knowledge with a greater appreciation for the subject. His leadership and management have helped create an exceptional environment that has encouraged me to become both a better student and researcher. I would attribute a significant amount of my educational and professional development to his direction.

I would like to thank my committee members Dr. Todd Lowe, Dr. Joseph Schetz and Dr. Jason Anderson for their insight and assistance in my research.

I would also like to thank all of my coworkers in Lab 7 for their assistance throughout my research career. Specifically I would like to thank Dr. Aurelien Borgoltz, Matt Rasnick, Mike Morton, Ryan Catlett, Manuj Awasthi and Dr. Nathan Alexander for all of their help in construction, installation and testing involved in this project. I could not have spent all of those long days in the wind tunnel or gotten as much done without their help. Their continued friendship outside of work has also helped keep me in good spirits throughout even the most stressful portions of my research.

I would like to thank Jacques $\mathrm{Li}$ and Tim Meyers in their extensive support in the development of the false wall panels and huge contributions to the roughness fabrication while undergraduate researchers. Tim's additional proof reading skills have helped me catch several sentences in this paper I was obviously writing while up late at night.

I would like to thank Bill Oetjens for his assistance in the tunnel and patience with the issues that arose during testing. A big thanks also goes out to everyone in the AOE machine shop. I would like to especially thank James Lambert whose ability to machine and manufacture large portions of the wall on a short notice kept many of the tunnel entries on time, and Scott Patrick whose design and CAD knowledge was indispensable.

Lastly I would like to thank the National Science Foundation for their financial support under grant CBET-0853674 that allowed this research to be conducted.

Thank you again to everyone for all your help and support,

-Jonathan Forest 


\section{Figure and Photographic Acknowledgements}

All figures and photographs presented in this paper were created or taken by the author unless otherwise noted. I would like to extend a special thanks for the individuals listed in the exceptions below.

Figure 2.1 - Schematic of Virginia Tech Stability Wind Tunnel. Adapted figure, original courtesy of AOE department. Used with permission.

Figure 2.2 - Schematic of Virginia Tech Stability Wind Tunnel, anechoic test section. Adapted figure, original from Devenport et al (2010). Used with permission.

Figure 2.9 - Stability Wind Tunnel Traverse System.

Photographer: Mike Morton (2011), used with permission.

Figure 2.18 - Microphone sleeves embedded in smooth test plate. Photographer: Manuj Awasthi (2011), used with permission. 
CHAPTER 1. INTRODUCTION ….................................................................................... 1

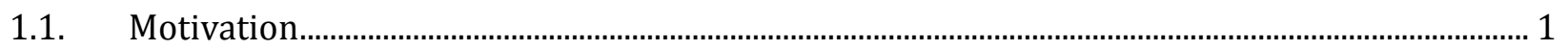

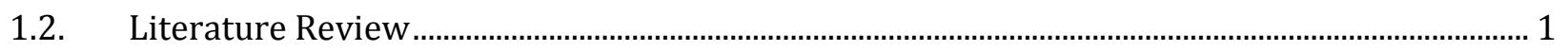

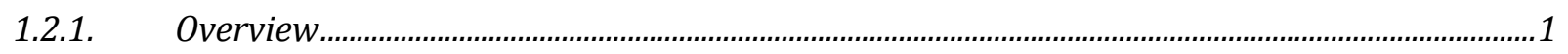

1.2.2. Smooth Wall Surface Pressure Fluctuations ...................................................................................1

1.2.3. Surface Roughness Description ….......................................................................................

1.2.4. Rough Wall Surface Pressure Fluctuations...............................................................................5

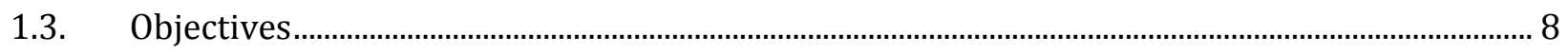

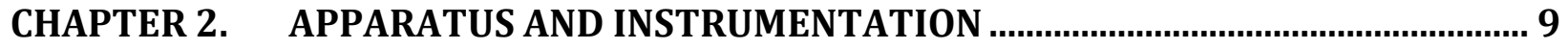

2.1. Virginia Tech Stability Wind Tunnel................................................................................................ 9

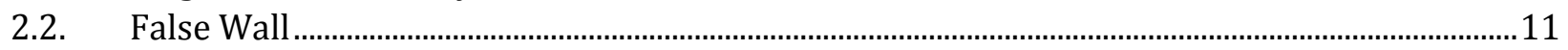

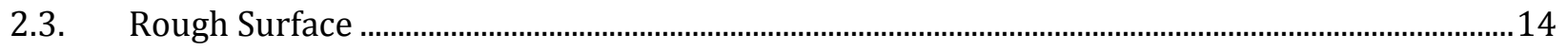

2.4. Boundary Layer Velocity Instrumentation ………......................................................................15

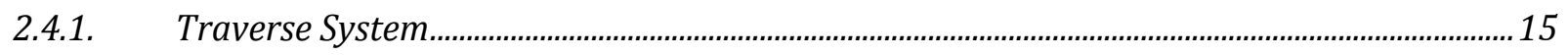

2.4.2. Flat Head Pitot Instrumentation …................................................................................................17

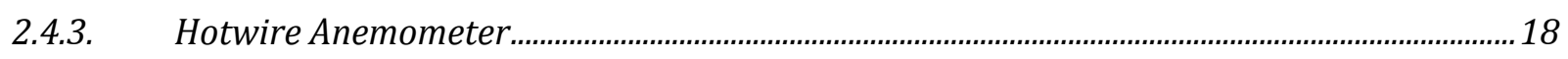

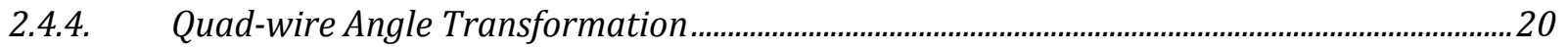

2.5. Surface Pressure Instrumentation....................................................................................................2 24

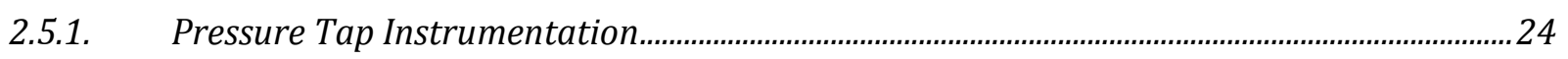

2.5.2. Wall Pressure Microphone Instrumentation .........................................................................24

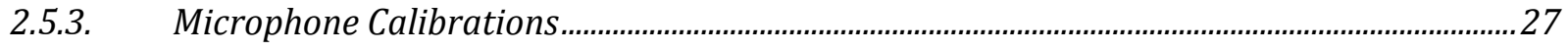

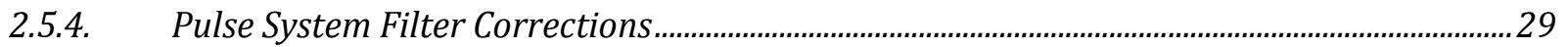

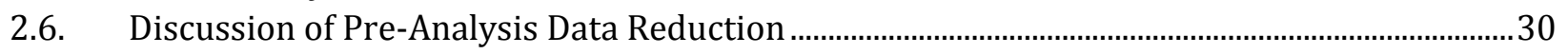

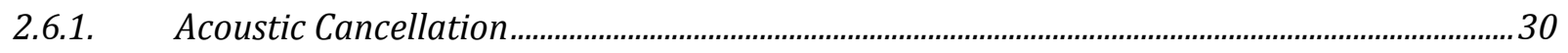

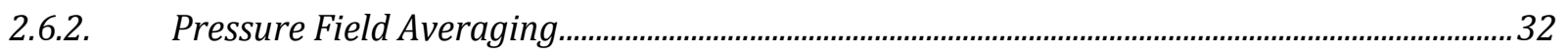

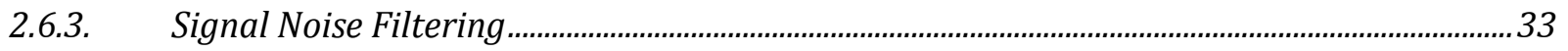

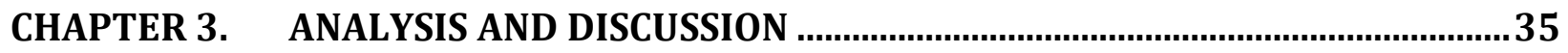

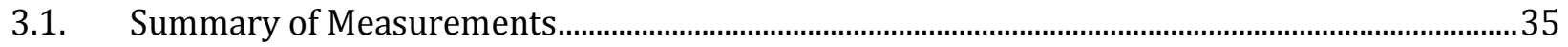

3.2. Mean Wall Pressure Gradient......................................................................................................... 35

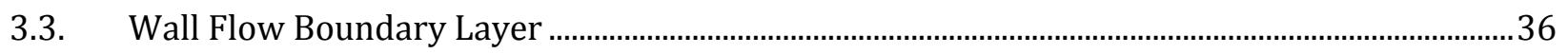

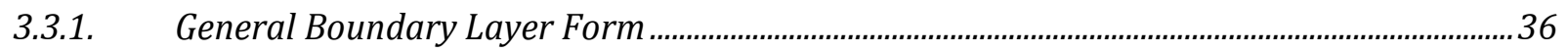

3.3.2. Boundary Layer Turbulence …………............................................................................. 45

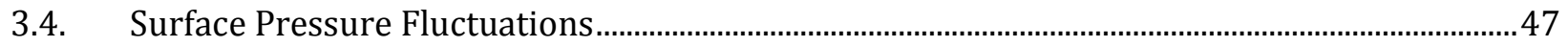

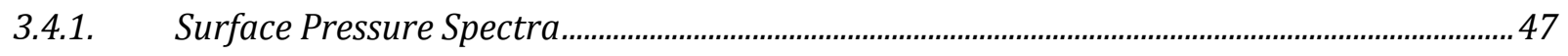

3.4.2. $\quad$ Surface Pressure Spectral Scaling and Features over Smooth Walls....................................49

3.4.3. Surface Pressure Spectral Scaling and Features over Rough Walls.........................................55

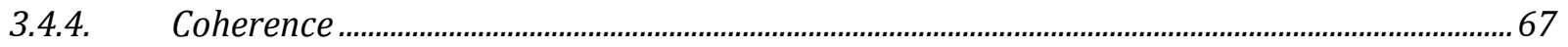

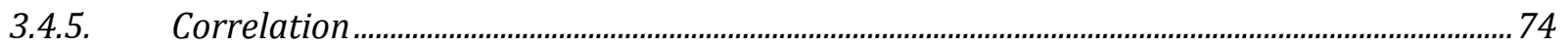

3.4.6. Phase, Phase Convection Velocity and Convection Velocity.......................................................82

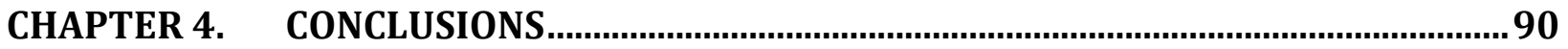

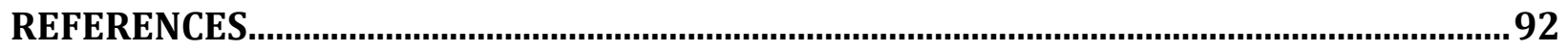




\section{Nomenclature}

\section{Roman}

\begin{tabular}{|c|c|}
\hline$A_{p}$ & Projected roughness surface area onto a plane normal to the flow direction \\
\hline$A_{s}$ & Wetted surface area of roughness as seen by the flow \\
\hline$c$ & Speed of sound \\
\hline$C_{f}$ & Skin friction coefficient \\
\hline$d$ & Pinhole diameter \\
\hline$d^{+}$ & Dimensionless pinhole diameter $\left(d^{+}=d u_{t} / v\right)$ \\
\hline$d_{e}$ & Mean distance between roughness elements \\
\hline$f$ & Frequency (Hz) \\
\hline$H$ & Boundary layer shape factor $\left(H=\delta^{*} / \theta\right)$ \\
\hline$k$ & Wavenumber in the streamwise direction, $\left(k=\omega / U_{c}\right)$ \\
\hline$k_{g}$ & Roughness height \\
\hline$k_{g}^{+}$ & Roughness height Reynolds number $\left(k_{g}{ }^{+}=k_{g} u_{t} / v\right)$ \\
\hline$k_{s}$ & Equivalent sand grain roughness height \\
\hline$k_{S}^{+}$ & Equivalent sand grain roughness height Reynolds number $\left(k_{s}{ }^{+}=k_{s} u_{t} / v\right)$ \\
\hline$p$ & Wall pressure \\
\hline$q$ & Dynamic pressure $\left(q=\frac{1}{2} \rho U_{e}^{2}\right)$ \\
\hline$R_{p p}$ & Space-time correlation \\
\hline$s$ & Separation distance \\
\hline$T K E$ & Turbulent kinetic energy $\left(T K E=\frac{1}{2} u^{\prime 2}+\frac{1}{2} v^{\prime 2}+\frac{1}{2} w^{\prime 2}\right)$ \\
\hline$T_{L}$ & Lagrangian timescale of space-time correlation decay \\
\hline$u$ & Local mean velocity, $x$-direction \\
\hline$u^{\prime}$ & Local RMS fluctuating velocity, $x$-direction \\
\hline$u^{+}$ & Normalized local mean velocity for the law of the wall, $x$-direction \\
\hline$U_{c p}$ & Phase convection velocity \\
\hline$U_{c}$ & Convection velocity \\
\hline$u_{t}$ & Friction velocity \\
\hline$U_{e}$ & Edge velocity \\
\hline$v$ & Local mean velocity, $y$-direction \\
\hline$v^{\prime}$ & Local RMS fluctuating velocity, $y$-direction \\
\hline$w$ & Local mean velocity, $z$-direction \\
\hline$w^{\prime}$ & Local RMS fluctuating velocity, $z$-direction \\
\hline$x$ & Streamwise position, measured downstream of primary trip \\
\hline$y$ & Horizontal position, measured away from false wall \\
\hline$y^{+}$ & Normalized distance away from the wall for the law of the wall \\
\hline$Z$ & Vertical position, measured down from tunnel centerline \\
\hline
\end{tabular}

\section{Greek}

$\begin{array}{ll}\alpha & \text { Phase angle } \\ \delta & \text { Boundary layer thickness } \\ \delta^{*} & \text { Boundary layer displacement thickness }\end{array}$




$\begin{array}{ll}\eta & \text { Spanwise separation } \\ \Gamma & \text { Coherence } \\ \kappa & \text { Von Kármán constant }(\kappa=0.40) \\ \nu & \text { Kinematic viscosity } \\ \omega & \text { Angular frequency (radians) } \\ \Phi & \text { Wall pressure spectral density } \\ \phi_{p p} & \text { Wall pressure spectrum } \\ \rho & \text { Density } \\ \tau & \text { Time delay } \\ \tau_{w} & \text { Wall shear } \\ \theta & \text { Boundary layer momentum thickness } \\ \xi & \text { Streamwise separation }\end{array}$




\section{List of Tables}

Table 2.1. Flow length scales and dimensionless pinhole size for $0.5-\mathrm{mm}$ pinholes under testing conditions. .............................................................................................................. 25

Table 2.2. Repeated microphone spacing locations. ................................................................. 32

Table 3.1. Boundary layer parameters for flow over a zero-pressure gradient wall.............. 40

Table 3.2. Interpolated boundary layer parameters used for wall pressure scaling in section 3.4 . 40

Table 3.3. Roughness parameters for flow over a zero-pressure gradient rough wall. Data from previous studies is also listed for comparison....................................................... 44

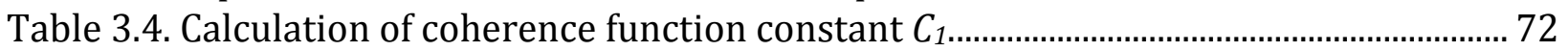

\section{List of Figures}

Figure 2.1. Schematic of the Virginia Tech Stability Wind Tunnel (adapted figure, courtesy of VT AOE department).

Figure 2.2. Schematic of Virginia Tech Stability Wind Tunnel anechoic test section with adjoining anechoic chambers. (Left) Typical anechoic test section setup. (Right) Modifications made to accommodate false wall. Figure adapted from Devenport et al (2010)... 10

Figure 2.3. Rail system installed in anechoic chamber to mount false wall panels................. 11

Figure 2.4. Typical false wall panel and frame schematic, non-flow side.................................. 12

Figure 2.5. Modified contraction and false wall installed in the anechoic test section, smooth

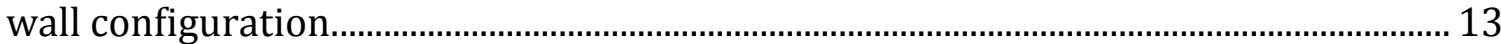

Figure 2.6. 19-mm and 9.5-mm trips........................................................................................ 13

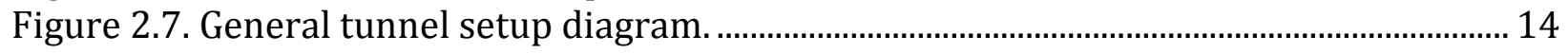

Figure 2.8. Installed 3-mm hemisphere deterministic rough surface. (Left inset) Hemisphere alignment at rough patch seams.............................................................. 15

Figure 2.9. Stability Wind Tunnel Traverse system. Photograph taken by Mike Morton (2011), used with permission................................................................................................. 16

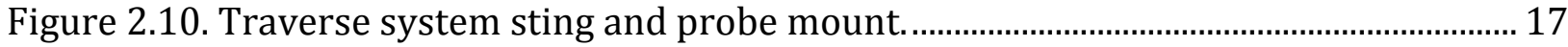

Figure 2.11. Flat head pitot. .......................................................................................................... 18

Figure 2.12. Four component hotwire anemometer (Quad-wire).............................................. 19

Figure 2.13. Single hotwire anemometer. …………………………………………………...... 19

Figure 2.14. Boundary layer turbulence data for high and low-angled probes, untransformed, measured at same conditions................................................................ 21

Figure 2.15. Boundary layer turbulence data for high and low-angled probes, transformed, measured at same conditions. ............................................................................................ 22

Figure 2.16. Quad-wire high angle probe correction transformation factor linear estimations................................................................................................................ 23

Figure 2.17. B\&K Type 4138-A-15 microphones with $1 / 2-\mathrm{mm}$ pinhole cap (left) and standard

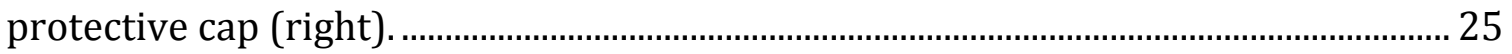

Figure 2.18. Two microphone sleeve types used, embedded in the clean surface test plate. Photograph taken by Manuj Awasthi (2011), used with permission............................. 26

Figure 2.19. Streamwise microphone placement diagram. Relative positioning of the microphones on the spanwise array is equivalent with a $90^{\circ}$ rotation......................... 26 
Figure 2.20. Microphone positions on the false wall for both smooth and 3-mm hemisphere

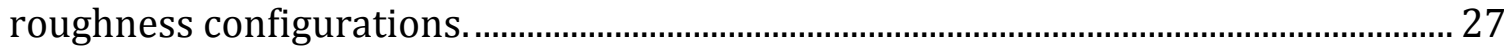

Figure 2.21. Microphone amplitude and phase calibration setup in anechoic chamber....... 28

Figure 2.22. Comparison between actual and fitted microphone calibrations for amplitude

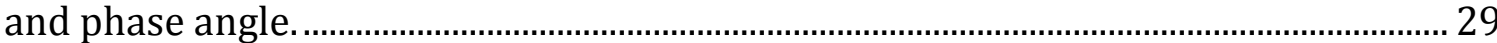

Figure 2.23. Pulse system filter correction factor.................................................................. 30

Figure 2.24. Background acoustic cancellation for select auto- and cross spectra................... 31

Figure 2.25. Auto- and cross spectral averaging for a select condition and microphone

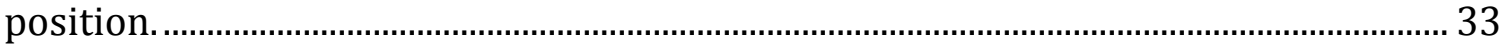

Figure 2.26. Surface pressure data with large signal noise subtracted....................................... 34

Figure 3.1. Mean surface pressure across hard wall testing surface.......................................... 36

Figure 3.2. Law of the wall for smooth and rough walls.............................................................. 39

Figure 3.3. Skin friction coefficient comparison to other zero-pressure gradient smooth

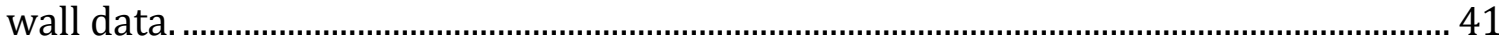

Figure 3.4. Boundary layer shape factor comparisons for (a) smooth and (b) rough wall

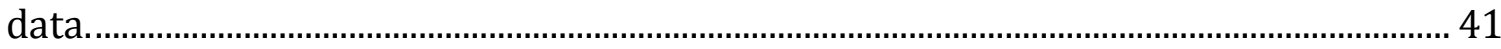

Figure 3.5. 3-mm roughness effects on boundary layer profile shape....................................... 42

Figure 3.6. Boundary layer two-dimensionality....................................................................... 43

Figure 3.7. Boundary layer turbulence intensity profiles for (a) smooth and (b) rough walls.

Figure 3.8. Boundary layer turbulent kinetic energy (TKE) comparison for rough and

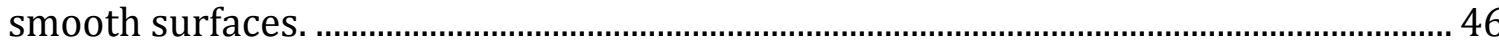

Figure 3.9. Boundary layer Reynolds shear stress profiles for (a) smooth and (b) rough

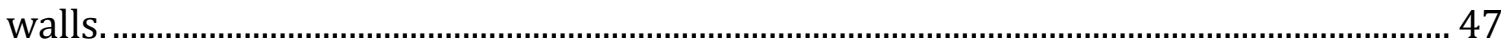

Figure 3.10. Wall pressure spectra for smooth walls using (a) streamwise and (b) spanwise microphone arrays....................................................................................................... 49

Figure 3.11. Wall pressure spectra for rough walls using (a) streamwise and (b) spanwise microphone arrays........................................................................................................ 49

Figure 3.12. Wall pressure spectra for smooth walls normalized on inner variable scaling.

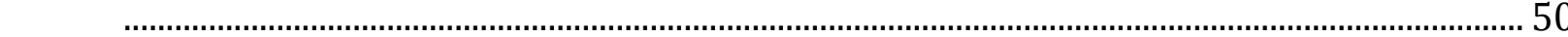

Figure 3.13. Wall pressure spectra for smooth walls normalized on (a) classical outer variable scaling and (b) mixed outer variable scaling.................................................... 54

Figure 3.14. Wall pressure spectra for rough walls normalized on Blake's (1970) roughness based inner variable scaling............................................................................................. 56

Figure 3.15. Wall pressure spectra for rough walls normalized on Varano's (2010) fully rough flow inner variable scaling............................................................................... 57

Figure 3.16. Wall pressure spectra for rough walls normalized on (a) smooth wall inner variables and (b) a modified Blake rough wall inner variable scaling..........................57

Figure 3.17. Wall pressure inner scaling variable location diagram. Objects are not to scale.

Figure 3.18. Wall pressure spectra for rough walls normalized on proposed roughness based inner variable scaling for (a) the present study only and (b) multiple studies

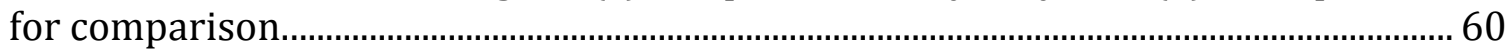

Figure 3.19. Wall pressure spectra from Smith (2008) for a range of roughness flows normalized on proposed roughness based inner variable scaling.................................. 61 
Figure 3.20. Wall pressure spectra for rough walls normalized on alternate roughness

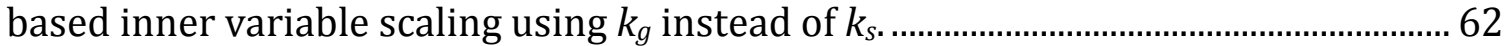

Figure 3.21. Wall pressure spectra for rough walls normalized on (a) classical outer variable scaling and (b) mixed outer variable scaling 65

Figure 3.22. Wall pressure spectra for smooth and rough walls overlaid, normalized (a) classical outer variable scaling and (b) mixed outer variable scaling.............................66

Figure 3.23. Smooth wall pressure spectra coherence for streamwise separations................ 68

Figure 3.24. Rough wall pressure spectra coherence for streamwise separations....................69

Figure 3.25. Smooth wall pressure spectra coherence for spanwise separations..................... 70

Figure 3.26. Rough wall pressure spectra coherence for spanwise separations...................... 71

Figure 3.27. Smooth wall pressure spectra coherence for fixed frequencies by phase angle.

Figure 3.28. Rough wall pressure spectra coherence for fixed frequencies by phase angle.74

Figure 3.29. Smooth wall pressure spectra correlation for streamwise separations. .............76

Figure 3.30. Rough wall pressure spectra correlation for streamwise separations................ 76

Figure 3.31. Smooth wall pressure spectra correlation and convective ridge for streamwise separations at 33.6-m/s................................................................................................ 77

Figure 3.32. Rough wall pressure spectra correlation and convective ridge for streamwise separations at 30-m/s.................................................................................................. 78

Figure 3.33. Smooth wall pressure spectra correlation for spanwise separations...................79

Figure 3.34. Rough wall pressure spectra correlation for spanwise separations.................... 79

Figure 3.35. Smooth wall pressure spectra correlation decay for streamwise separations at $22.4,33.6,44.8,56.0$ and $67.2-\mathrm{m} / \mathrm{s}$. 81

Figure 3.36. Rough wall pressure spectra correlation decay for streamwise separations at

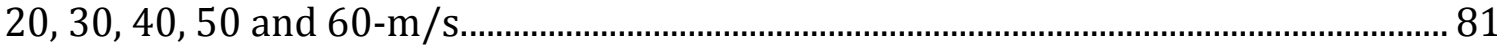

Figure 3.37. Smooth wall pressure spectra phase angle for streamwise separations............. 83

Figure 3.38. Rough wall pressure spectra phase angle for streamwise separations................ 83

Figure 3.39. Smooth wall pressure spectra phase angle for spanwise separations. ................ 84

Figure 3.40. Rough wall pressure spectra phase angle for spanwise separations.................... 84

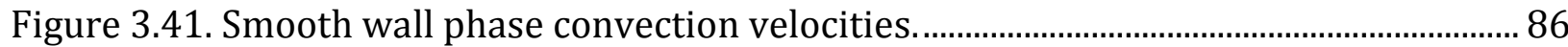

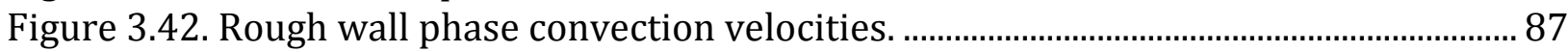

Figure 3.43. Convection velocities for (a) smooth and (b) rough walls..................................... 89 


\section{CHAPTER 1. INTRODUCTION}

\subsection{Motivation}

Many large ships, aircraft and structures operate under or encounter high Reynolds number flows on a regular basis. Surface roughness can often be found on these vehicles and structures being due to a manufacturing or environmental process, and in some cases being the result of an exposed design feature. Regardless of the reason, the surface roughness will promote the formation of a turbulent boundary layer and a fluctuating pressure field. It has long been known that the interaction between the surface roughness and the turbulent boundary layer alters the scaling and character of the wall pressure field, though the exact nature of this interaction is still somewhat unknown. Only a limited number of experimental studies have been conducted investigating the effects of surface roughness on the wall pressure field. Due to the difficulties in obtaining high Reynolds number flows over fully rough surfaces in laboratory settings, a majority of these studies were unable to investigate this phenomenon under flow conditions predicted to be fully free of transitional effects that would ensure that similarity laws would be observed. This thesis thus presents the efforts to scale and describe the wall pressure spectrum of a rough wall, high Reynolds number, turbulent boundary layer flow free of transitional effects using the Virginia Tech Stability Wind Tunnel facility.

\subsection{Literature Review}

\subsubsection{Overview}

The wall pressure spectra developed over zero-pressure gradient smooth surfaces located beneath turbulent boundary layer flows have been the subject of a number of experimental studies. While this work has uncovered the major characteristics and behaviors of these pressure fields, the underlying mechanisms controlling these features are still somewhat debated. Furthermore, as few studies have focused on the effects of roughness on the wall pressure field, a review of what is known about the smooth wall pressure field is beneficial. This review is thus intended to cover the major findings associated with the smooth wall pressure field, examine the basic principles associated with surface roughness, and survey relevant rough wall pressure fluctuation studies. Note that only rough wall pressure fluctuation studies were considered where at least one flow condition was found to be fully rough while maintaining that the roughness elements did not constitute a major portion of the boundary layer thickness.

\subsubsection{Smooth Wall Surface Pressure Fluctuations}

The wall pressure fluctuations beneath turbulent boundary layers have been studied since the 1950's in an effort to determine their characteristics. A review is provided by Bull (1996) which provides insight into the general findings and theories regarding the fluctuating pressure field. In general for turbulent wall bounded flows, the fluctuation pressure is influenced by the integrated velocity field, inclusive of the entire range of the boundary layer. The turbulence structures contributing to the fluctuating pressure are largely convected by the local mean velocity, which varies throughout the boundary layer. 
Thus similar to what is seen for the mean flow boundary layer, the different regions of the wall pressure frequency spectra also collapse on distinct scaling factors.

Bull (1967) was one of the early experimental studies investigating the wall pressure fluctuation field beneath turbulent boundary layers for smooth surfaces in which a sufficiently small pressure transducer was used to capture the high frequency pressure spectra. In this study, Bull found that the turbulence eddies could be assumed to be 'frozen', meaning that all turbulent structures associated with a particular wavenumber could be treated as having a single convection velocity. As pointed out by Bull (1996), this assumption was commonly used throughout the early literature. More recent studies including Morrison et al. (1992) and briefly mentioned in Bull (1996) however have shown that the turbulent eddies are not completely frozen, especially in the outer portions of the boundary layer. Thus while this is one of the dominant behaviors of the turbulent eddies, a mild amount of variation from this pattern can be observed. Still, each convection velocity can generally be assumed to be related to a particular boundary layer location. This implies the turbulence structures tend towards a constant boundary layer location. The high wavenumber (and thus high frequency) pressure spectra are linked with the inner boundary layer regions, while the low wavenumber and low frequency spectra are identified with the larger turbulence eddies found in the outer regions of the boundary layer. As reviewed by Bull (1996), several studies have revealed that for zero-pressure gradient turbulent boundary layer flows over smooth surfaces, the wall pressure frequency spectra can be divided into four distinct regions at high, medium, low and very low frequencies.

At the highest frequencies of the pressure spectra, numerous studies including Farabee and Casarella (1991), Goody (2004) and Gravante et al. (1998) show a sharp roll off. This region is attributed to turbulent eddies located in the innermost portions of the boundary layer. As such, this region is typically shown to collapse when scaled on inner boundary layer variables kinematic viscosity $v$, friction velocity $u_{t}$, and wall shear $\tau_{w}$. Studies have shown that this region normally exhibits a frequency power law dependence of $\omega^{-5}$ at the highest frequencies. It has, however, been pointed out by sources including Bull (1996), Gravante et al. (1998) and Schewe (1983), that if the transducer sensing diameter is too large, spectral attenuation can occur, specifically affecting this region. Additionally, Schewe was also able to show that a small frequency band at the beginning of the roll off region should exhibit a $\omega^{-2.3}$ dependence, consistent with the locally isotropic turbulence found in portions of the boundary layer buffer region for smooth walls.

In the mid frequency range of the wall pressure spectrum, an overlap or 'universal' region can be found which is largely attributable to the turbulent structures located in the logarithmic region of the boundary layer. Bradshaw (1967) was an early proponent showing this region experiences influences from both the inner and outer portions of the boundary layer. It was argued that since both inner and outer boundary layer variables (discussed below) could collapse the pressure spectrum in this region, dimensional analysis should require an inverse wavenumber $\left(k^{-1}\right)$ dependence. A later study by Panton and Linebarger (1974) also came to this conclusion while creating a theoretical wavenumber pressure spectrum model based on a variation of Lighthill's equation and theoretical boundary layer scaling properties. While these two studies theorized as to what equated to the existence of an overlap region with a $\omega^{-1}$ dependence, experimental results have not always supported such a claim. Blake 1970, Farabee and Casarella 1991, and 
McGrath and Simpson 1987 are among many of the studies who find the overlap region to have exhibit a lower frequency dependence, usually between $\omega^{-0.7}$ and $\omega^{-0.83}$. Goody (2004) showed that this region's length is Reynolds number dependent, with larger Reynolds number flows exhibiting significantly broader overlap regions.

The low frequency pressure spectrum is largely due to turbulent structures in the outer portions of the boundary layer. As pointed out by Bull (1996) and Goody (2004), several different scaling factors have been shown to collapse the low frequency spectra. In this region the most commonly used variables to collapse the data incorporate a classical scaling using boundary layer thickness $\delta$, friction velocity $u_{t}$, and wall shear $\tau_{w}$; a scaling based on dynamic pressure $q$, boundary layer displacement thickness $\delta^{*}$, and freestream or edge velocity $U_{e}$; and a mixed scaling implementing wall shear $\tau_{w}$, boundary layer displacement thickness $\delta^{*}$, and edge velocity $U_{e}$. Bull notes that several other combinations of these outer scaling variables have been used to successfully collapse the low frequency spectra, though asserts that the classical variable scaling seems to be the most reliable. This is in agreement with Goody (1999), who found a low collapse for several scaling factors as long as $\tau_{w}$ was used to scale the pressure amplitude.

At very low frequency region, the experimental study by Farabee and Casarella (1991) found the pressure spectra to collapse on the outer boundary layer scaling based on dynamic pressure $q$, boundary layer displacement thickness $\delta^{*}$, and edge velocity $U_{e}$. While this is the only experimental study found that reached low enough frequencies to observe this region and show a $\omega^{2}$ dependence, Bull (1996) pointed out that the Kraichnan-Phillips theory for finding the wall pressure spectra from the Navier-Stokes equation had previously suggested a $\omega^{2}$ dependence at low frequencies for smooth wall zero-pressure gradient flows. Following Bull, it has also been theorized that there may be another region at even lower frequencies wherein the pressure spectrum should become asymptotic with some constant. However, as explained by Bull, the high, medium and low frequency regions combine to account for the majority of the mean-square pressure, with the lowest frequency region or regions contributing very little.

The progression of the pressure field can be studied by analyzing the space-time correlation and pressure coherence functions. Many of the major findings in this area were initially covered by Bull (1967). In this study, it was found that the high frequency turbulent structures in the inner portions of the boundary layer were the dominant contributors to the coherence of the pressure field over short distances, while the lower frequency structures came to dominate the pressure field coherence over larger distances. This was shown to occur as the higher frequency eddies rapidly lost coherence for larger separations. It was later confirmed by Farabee and Casarella (1991) and others listed in Bull (1996) that the pressure fluctuations in these regions decayed at approximately equal proportions to their wavelengths. At the lower scaled frequencies associated with turbulence structures located in the outer portions of the boundary layer, the pressure fluctuations were often found to decay more rapidly than a dependence on wavelength would imply. These turbulence structures were found to be losing coherence due to the increased mixing in the outer portions of the boundary layer over the large separations these structures were being convected. Despite this however, the mean convection velocity of the correlated turbulent structures was shown to increase for larger separations since the larger eddies became the predominant sources of coherent pressure convected over these large separations. 


\subsubsection{Surface Roughness Description}

The presence of surface roughness can have a profound effect on the boundary layer. Following Jiménez's (2004) review, the most apparent effects of the surface roughness on the mean flow of the boundary layer is a shift in the velocity profile and an increase in the skin friction coefficient $C_{f}$ when compared to the smooth wall flow. Roughness also has an effect on the turbulence structure of the velocity field, though beyond realizing an increase in the streamwise velocity fluctuations in the logarithmic region of the boundary layer, many of the conclusions are still not definitive for a wide range of cases. It should be noted that there are two main classifications of roughness categories, namely $k$-type and $d$-type. This study will only briefly review $k$-type roughness. The $d$-type roughness where the elements are spaced closely enough that portions of the fluid becomes isolated and recirculate in the crevices, reducing the effect of roughness height, will be excluded.

One commonly used term for describing surface roughness is the equivalent sand grain roughness $k_{s}$. As described by Schlichting (1979), this variable can be used as a comparative roughness measurement based on the experiments done by J. Nikuradse in 1933 for sand roughened pipe flows. Equivalent sand roughness can be defined for a wide range of roughness types and configurations as it exhibits more of the characteristics of an aerodynamic measurement rather than a geometric height. Specifically, it is defined by how the roughness shifts the inner scaled mean velocity away from the law of the wall for smooth surfaces. When scaled on friction velocity $u_{t}$ and kinematic viscosity $v$, the equivalent sand grain roughness Reynolds number $k_{s}{ }^{+}=k_{s} u_{t} / v$ can be used as a good indication of the amount of interference the roughness has on a particular flow.

For small values $\left(k_{s}{ }^{+}<5\right)$, the roughness effects are generally considered to be contained to within the laminar sublayer of the boundary layer, and the surface can be said to be hydraulically smooth. Jiménez (2004) points out, however, that while this value is generally the accepted limit, it is somewhat dependent on the type roughness being used. Following Schlichting (1979), for larger values of $k_{s}{ }^{+}$, the roughness begins to interfere with the flow beyond the laminar sublayer, with the equivalent roughness height protruding into the buffer and initial portions of the logarithmic region. This flow regime is said to be transitionally rough. An important distinction here is that while the equivalent roughness height protrudes into the mean flow beyond the extent of the laminar sublayer, the laminar sublayer itself is not viewed as being fully destroyed in this regime. When the roughness is large enough such that $k_{s}{ }^{+}>70$, however, the laminar sublayer is destroyed and the flow is said to be fully rough. For fully rough flows, the form drag on the roughness elements becomes a dominant feature and unlike smooth and transitionally rough flows, the inner regions of the boundary layer become independent of viscosity.

It is important here to note the difference between the calculated equivalent sand grain roughness height $k_{s}$ and physical roughness height $k_{g}$. While the equivalent sand grain roughness height can be viewed as a comparative aerodynamic measurement and generally takes into account surface sparseness, it is not an accurate measure of how far into the boundary layer the roughness elements may locally affect the flow. From Jiménez (2004), the boundary layer height to roughness height ratio, or blockage ratio $\delta / \mathrm{kg}_{\mathrm{g}}$, needs to be large enough to prevent the roughness from directly affecting large portions of the boundary layer logarithmic region. Since a large portion of the turbulent energy produced in a boundary layer occurs in the logarithmic region, even sparsely spaced elements taking 
up a non-insignificant fraction of the boundary layer height can alter the turbulent production of the entire flow. Jiménez suggests that even with conservative estimates for the reach of the roughness's effects in the boundary layer, it would require blockage ratios of at least $\delta / \mathrm{kg}_{\mathrm{g}}>40$ before any sort of similarity laws could be observed. It is contended that larger ratios may be required in practice. Jiménez proposes that boundary layers with lower blockage ratios should instead be treated as flows over obstacles rather than surface roughness, as lower blockage ratios become increasingly dependent on roughness geometry and spacing.

In order to ensure that flows over rough surfaces are completely free of transitional effects thus allowing for similarity laws to be observed, Jiménez's (2004) suggests in his review that the flow should to meet two specific conditions. In order to fully verify that direct roughness effects are not a significant factor in the logarithmic region, a condition of $\delta / k_{g}>50$ is suggested. Additionally, to guarantee the rough flow is fully developed, a roughness height Reynolds number of $\mathrm{kg}^{+}>80$ is proposed. Under these conditions it is thought that any roughness specific results would disappear allowing for broader, more universal conclusions to be drawn regarding surface roughness.

\subsubsection{Rough Wall Surface Pressure Fluctuations}

The study by Aupperle and Lambert (1970) was one of the first investigations focusing on surface pressure fluctuations for fully rough flows. Measurements were taken in a continuous-flow subsonic wind tunnel at $50-\mathrm{m} / \mathrm{s}$ for one smooth and three roughness configurations. Each roughness configuration was comprised of either commercial grade 36, 12 or 4 grit roughness elements uniformly spaced with a constant particle size to particle separation ratio along a removable panel. Under the flow conditions tested, these corresponded to equivalent sand grain roughness Reynolds numbers between of $\mathrm{kg}^{+}=81$ and $k_{g}{ }^{+}=858$ and blockage ratios between $\delta / k_{g}=56$ and $\delta / k_{g}=10$ respectively. An effort was put forth to determine the effect of the placement of the pressure transducers in relation to the roughness elements, though only minor spectral variation was observed as long as the transducer was not located directly behind a roughness element.

From their data, Aupperle and Lambert showed that the wall pressure spectral densities exhibited a strong dependence on roughness size. While they found no direct relationship between smooth and rough wall spectral densities, they did attempt to relate their spectra over the three different rough wall configurations using an empirically determined scaling. They found some measure of collapse when using the scaling factors $k_{s} / u_{t}$ in the frequency domain and $1 /\left(\tau_{w}^{2} C_{f}\right)$ for pressure spectral levels. While an outer variable scaling was not attempted in this study, these variables suggest inner boundary layer scaling. Interestingly, these proposed scaling factors showed the best collapse only along the low and mid frequency range while the expected high frequency roll off showed a less complete collapse. It should be noted however that Aupperle and Lambert did not specifically intend to find inner or outer variable scaling, and rather set out to merely find some generic normalization to relate the wall pressure spectral densities observed over their different roughness configurations.

Aupperle and Lambert only examined the space-time correlations and convection velocities for two roughness configurations, corresponding to the configurations using grades 4 and 12 roughness grit. However, it was found that the space-time correlations varied almost exclusively due to the differences in convection velocities, which showed 
only a minor dependence on roughness configuration. Aupperle and Lambert stated that at low frequencies, the associated turbulent eddy convection velocities were largely unaffected by the roughness configuration. The only difference in correlation found at low frequencies between smooth and rough walls was a more rapid decay in correlation due to roughness. At high frequencies however, it was shown that the associated turbulent eddies were largely destroyed by the roughness elements. They did not find any high frequency correlation whenever a roughness particle was placed between measurement locations.

Blake (1970) also performed an early investigation of the wall pressure fluctuations for both rough and smooth walls. Measurements were taken in a subsonic acoustic wind tunnel at flow speeds between 22 and $50-\mathrm{m} / \mathrm{s}$ for smooth surfaces and between 38 and 50 $\mathrm{m} / \mathrm{s}$ for rough surfaces. Three types of roughness configurations were used by attaching roughness elements to a removable plywood panel with the configurations corresponding to equivalent sand grain roughness Reynolds numbers between of $k_{g}{ }^{+}=172$ and $k_{g}{ }^{+}=412$ and blockage ratios between $\delta / k_{g}=26$ and $\delta / k_{g}=42$.

At low frequencies, Blake found the rough wall pressure spectra scaled on mixed outer variables similar to what is found for smooth walls. It was thus inferred that the pressure sources associated low frequency fluctuations were similar to the smooth wall pressure sources, though no conclusions were drawn on the exact nature of this turbulence production. In order to obtain a high frequency collapse, Blake attempted to determine a roughness based inner variable pressure scaling. Blake argued that when scaled on outer variables, the rough wall pressure spectra differed from the smooth wall pressure spectra based on the Strouhal number $\omega k_{g} / u_{t}$. This led to the determination of a roughness based inner scaling using $k_{g} / u_{t}$ in the frequency domain and $1 /\left(\tau_{w}^{2}\right)$ for pressure spectral levels. Blake's results showed an improved mid and high frequency spectral collapse, though at high frequencies there appeared to be some variation between some of the roughness configurations. At the time, this was attributed to differences in roughness element spacing ratios. Blake thus concluded that the high frequency pressure spectrum was primarily determined by wake shedding off the top of roughness elements, with a slight dependence on element spacing.

In Blake's paper, the effects of surface roughness on the two point pressure fluctuation measurements were also investigated. Blake found that the surface roughness had the effect of lowering the convection velocity, asserting that it was proportional to the mean velocity defect due to roughness. In addition it was found that the coherence loss was similar over rough and smooth walls for outer boundary layer flow structures. The coherence loss for the inner boundary layer regions, however, was found to be much higher over rough surfaces. This was attributed to the difference in turbulence production near the wall, which was concluded to be dependent on roughness height over rough walls rather than the viscous lengths associated with smooth wall flows. In general Blake concluded that for rough walls, roughness had the largest influence on medium and small scale turbulence structures, while unlike the findings of Aupperle and Lambert (1970), roughness spacing was speculated to have mild influence on the larger turbulence structures.

Blake's data from 1970 was revisited in Blake's 1971 paper. During this symposium, Blake reviewed the data from both Blake (1970) and Aupperle and Lambert (1970). It was found that both data sets showed a measure of collapse when using the earlier scaling 
proposed in Blake (1970). Additionally it was argued that this scaling was less empirically determined and could be more universally applied. While this was an important finding, some minor spectral dispersion was still apparent especially for the 36 grit roughness from Aupperle and Lambert's study.

Smith (2008) studied the fluctuation pressure field for a wide range of transitionally and fully rough flows, however none of the fully rough flows met Jiménez's (2004) suggested criteria as listed at the end of section 1.2.3. These measurements were taken in a wall jet wind tunnel facility such that the medium and low frequency spectra associated with the outer boundary layer pressure field would not be representative of a conventional turbulent boundary layer flow over rough or smooth surfaces. However as described in this paper, these results should generally be applicable to more conventional studies in regards to the findings derived from the inner regions of the boundary layer.

The relevant results determined in Smith largely revolve around the collapse of the wall pressure spectra over rough walls. A moderate collapse of Smith's data was observed while using the scaling variables proposed by Aupperle and Lambert's (1970) and Blake (1970) for equivalent roughness configurations. When using different roughness configurations, these scaling variables were shown to exhibit a poor collapse for the entire range of roughness data. It was identified, however, that as flows reached larger values of roughness Reynolds numbers near $k_{s}{ }^{+}>80$, the spectra did begin to come closer to reaching an agreeable collapse. Smith speculated that an inner region spectral collapse could occur at larger roughness Reynolds numbers while using these scales, though it should be noted that very few roughness cases resided in this range. While a wide range of roughness grits were tested between 20 grit and 220 grit, only the roughness configurations using 20,36 and 40 grit showed signs of possible collapse.

In an attempt to fully collapse his data, Smith proposed an empirical roughness based inner scaling using $k_{s} /\left[u_{t}\left(k^{+}\right)^{0.825}\right]$ in the frequency domain and $1 /\left[\tau_{w}^{2} C_{f} R e_{\delta}^{0.5}\left(k^{+}\right)^{2.15}\right]$ for pressure spectral levels. This scaling was found to perform well in aligning the frequency domains for the roughness configurations and flow conditions tested, though it had difficultly normalizing the spectral levels. While not discussed in Smith's paper there was some evidence that the quality of this scaling progressively deteriorated as the roughness based Reynolds number was increased. Such a behavior would suggest that these variables were either scaling on a dominant geometric feature specific to the sandpaper roughness configurations tested, or that this scaling is more suited to flows in the transitionally rough regime. In either case, Smith's results suggested that a universal inner variable roughness scaling may not have yet been found for the pressure spectra in either his paper or those he reviewed.

Varano (2010) examined the turbulent boundary layer flow over rough surfaces, including an investigation of the rough wall pressure field. In this experiment Varano tested 1-mm radius hemispheres spaced at either 4, 5.5, 8 or 11-mm separations for a square array pattern. These surfaces were tested in the Virginia Tech Small Boundary Layer Wind Tunnel with measurements taken for each configuration at $20-\mathrm{m} / \mathrm{s}$ and $27-\mathrm{m} / \mathrm{s}$ flow speeds resulting in fully rough flows for each configuration. For wall pressure fluctuations, only single point measurements were taken with a second pressure transducer used only for acoustic cancellation. 
Varano's results show a high frequency collapse for his data sets when using Blake's (1970) roughness scaling. Varano showed that for flows in the fully rough regime, the friction velocity $u_{t}$ should be proportional to the edge velocity $U_{e}$. Blake's scaling was then rewritten and applied by using $k_{g} / U_{e}$ in the frequency domain and $1 /\left(\rho^{2} U_{e}^{4}\right)$ for pressure spectral levels. The assumptions in this scaling were only valid for fully rough flows, though they showed comparable collapse to Blake's proposed scaling. From this collapse, Varano countered Blake's claims and postulated from his results that element spacing was not an important factor for high frequency scaling. However, an overlap region was observed, though a mid-frequency collapse was not observed for either set of scaling parameters. Varano continued and applied both Aupperle and Lambert's (1970) proposed empirical scaling and Smith's (2008) proposed empirical scaling. These results both proved to show a poor collapse of Varano's data. Interestingly however, both sets of scaling appeared to separately collapse the two densely packed roughness configurations and the two sparsely packed roughness configurations. Still, these results suggested that both these scaling parameters were not universally applicable to multiple roughness configurations. Varano also attempted a low frequency collapse using classical outer boundary layer scaling. While no collapse was observed, results were said to be inconclusive due to the possible interaction between the tunnel floor and ceiling boundary layers.

\subsection{Objectives}

The aim of this study is to investigate the effects of surface roughness on the wall pressure fluctuations of high Reynolds number turbulent boundary layer flows. The wall pressure spectra were measured over both smooth and rough walls in the Virginia Tech Stability Wind Tunnel using streamwise and spanwise microphone arrays. The rough wall configuration used in this study was composed of 3-mm hemispherical elements arranged in a $16.5-\mathrm{mm}$ square array laid in a continuous pattern over a $1.76 \times 7.32-\mathrm{m}$ surface. A range of flow conditions were examined, with each of the rough wall flows residing in the fully rough regime while retaining low boundary layer blockage ratios due to roughness height. The objectives of this study are as follows:

- Investigate the wall pressure spectra inner variable scaling for fully rough flows at high Reynolds numbers in order to determine the factors associated with rough wall, high frequency turbulence structures.

- Examine outer variable wall pressure spectra scaling to investigate the effects of surface roughness on the turbulence structures located in the outer regions of the boundary layer.

- Determine the existence of an overlap region for the rough wall pressure spectra at high Reynolds numbers, and investigate its characteristics and implications if present.

- Examine the effect of roughness on the decay of surface pressure fluctuations.

- Investigate how surface roughness affects the convection velocity of the surface pressure fluctuations. 


\section{CHAPTER 2. APPARATUS AND INSTRUMENTATION}

\subsection{Virginia Tech Stability Wind Tunnel}

The data for this study was collected in the Virginia Tech Stability Wind Tunnel, which has an overhead schematic as shown below in Figure 2.1. This subsonic wind tunnel utilizes a continuous, single return design and is capable of achieving speeds in excess of $80-\mathrm{m} / \mathrm{s}$ in the test section. Upgrades to the test section and wind tunnel circuit (Smith, et al. 2005, Crede 2008, Remillieux, et al. 2008) allow this facility to run in an anechoic configuration that has been shown to be acoustically quiet while still maintaining favorable aerodynamics (Remillieux, et al. 2008, Devenport, et al. 2010). The flow entering the test section has been found to have turbulence levels of $0.021 \%$ at $21-\mathrm{m} / \mathrm{s}$ and $0.031 \%$ at 57 $\mathrm{m} / \mathrm{s}$.

The tunnel is powered by a $0.45-\mathrm{MW}$ variable speed DC motor capable of drawing up to 900 Amps. The motor is controlled by a custom designed Emerson VIP ES-6600 SCR Drive, which is regulated to limit any cyclic unsteadiness produced by the drive system. The motor is directly connected to the fan, which measures $4.27-\mathrm{m}$ in diameter. The fan consists of 8 Clark-Y airfoil section blades and can be driven at over 600-RPM. A foam fan liner has been applied to reduce the tip gap and tip noise by almost 6- $\mathrm{dB}$ across the entire frequency range (Remillieux, et al. 2008).

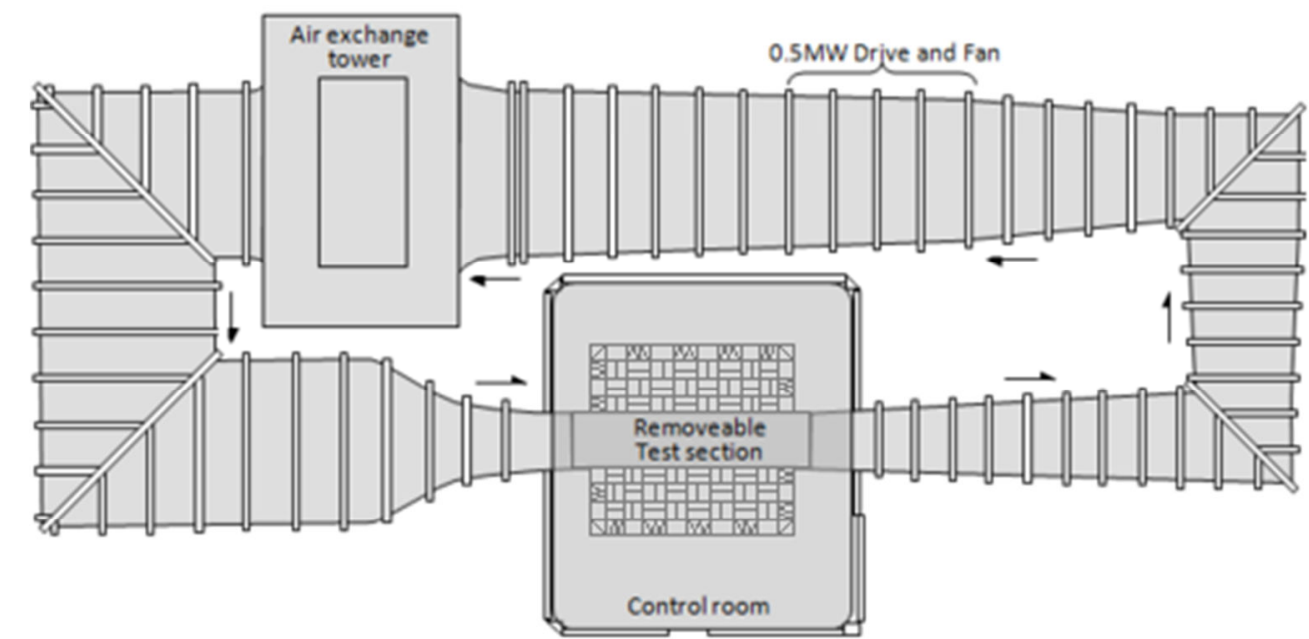

Figure 2.1. Schematic of the Virginia Tech Stability Wind Tunnel (adapted figure, courtesy of VT AOE department).

Downstream of the fan, the flow enters an air exchange tower which is partially open to the atmosphere. This design allows for the stabilization of the flow with the ambient atmospheric temperature. After the exchange tower, the flow is turned 180 degrees by two sets of turning vanes. The upstream set of turning vanes nearest the exchange tower has been lined with melamine foam on the pressure side of the vanes to act as an acoustic baffle facing the fan and motor. After both sets of turning vanes, the flow is directed into a $5.5 \times 5.5-\mathrm{m}$ square settling chamber. The floor and walls of the settling 
chamber have been coated with a 50-mm thick urethane foam liner used as an acoustic treatment. Additionally, the settling chamber contains 7 turbulence reduction screens with a $60 \%$ open air ratio. After passing through the turbulence reduction screens, the flow is accelerated by a 9:1 ratio contraction nozzle into the test section.
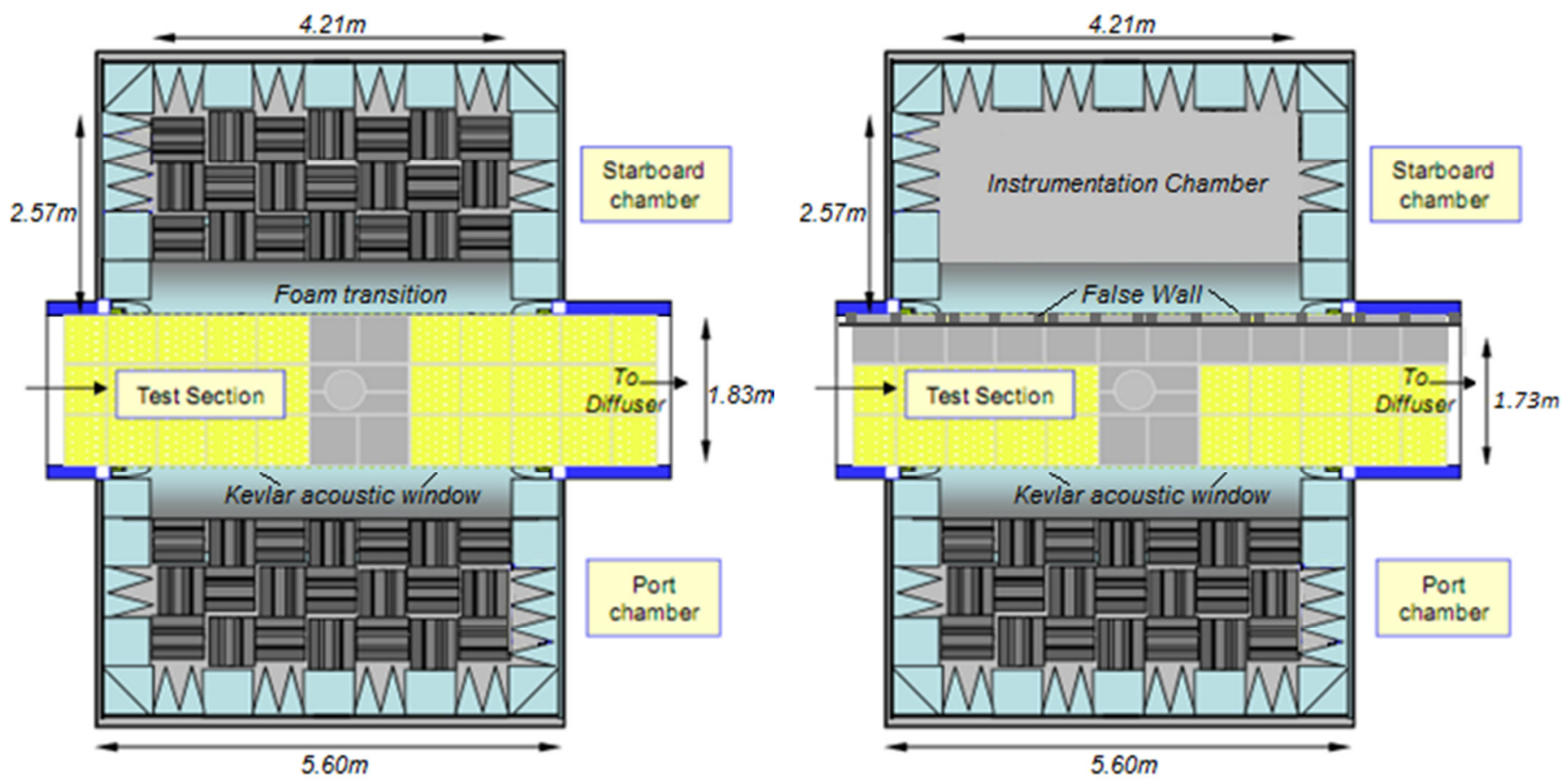

Figure 2.2. Schematic of Virginia Tech Stability Wind Tunnel anechoic test section with adjoining anechoic chambers. (Left) Typical anechoic test section setup. (Right) Modifications made to accommodate false wall.

Figure adapted from Devenport et al (2010).

The Virginia Tech Stability tunnel is capable of using either an aerodynamic, hardwall test section or an anechoic test section. Initial tests for this study were made using the aerodynamic test section, though subsequent runs, including all data presently presented, was collected while using the anechoic test section. The anechoic test section, shown in Figure 2.2, has a square $1.83-\mathrm{m}$ cross section and is $7.3-\mathrm{m}$ long. The floor and ceiling of the test section are acoustically treated with foam wedges, and covered by perforated aluminum panels overlaid with stretched Kevlar. The stretched Kevlar screens act as semipermeable surfaces that guide the flow, while remaining acoustically transparent (Devenport, et al. 2010). Acoustically treated $2.57 \times 4.21-\mathrm{m}$ anechoic chambers are typically adjoined to both sides of the anechoic test section, separated only by the stretched Kevlar windows. For this study, the starboard Kevlar window was replaced with a false wall test surface, which is described below in section 2.2. The addition of the false wall reduced the test section's nominal width to $1.73-\mathrm{m}$ as seen at the right in Figure 2.2.

After exiting the test section, the flow encounters 16 vortex generators evenly spaced around the tunnel's perimeter. These lead into the 3 degree tunnel diffuser which has been lined on the side walls and ceiling with $50-\mathrm{mm}$ thick melamine foam designed to reduce high frequency noise generated from the fan and motor from traveling upstream into the test section. After the diffuser, the flow encounters two more sets of turning vanes, and is recirculated back to the fan. 


\subsection{False Wall}

The anechoic test section was modified to accommodate a false wall test surface on which to grow large, high Reynolds number turbulent boundary layer flows. As seen at the right in Figure 2.2, the starboard Kevlar wall was removed from the anechoic test section and replaced by six modular framed Lexan panels.

In order to install the false wall panels, the 24 perforated aluminum-Kevlar panels nearest the starboard wall on the floor and ceiling were replaced with equivalent sized solid aluminum plates. Railing mounts were then secured along the floor and ceiling of the anechoic test section. The floor rails used to attach the false wall panels are shown in Figure 2.3. These railings attached to the wall panels through the use of right angle brackets, allowing each wall panel to be adjusted to obtain a zero-pressure gradient flow and slide in the lateral (into the flow) direction by up to $0.1-\mathrm{m}$.

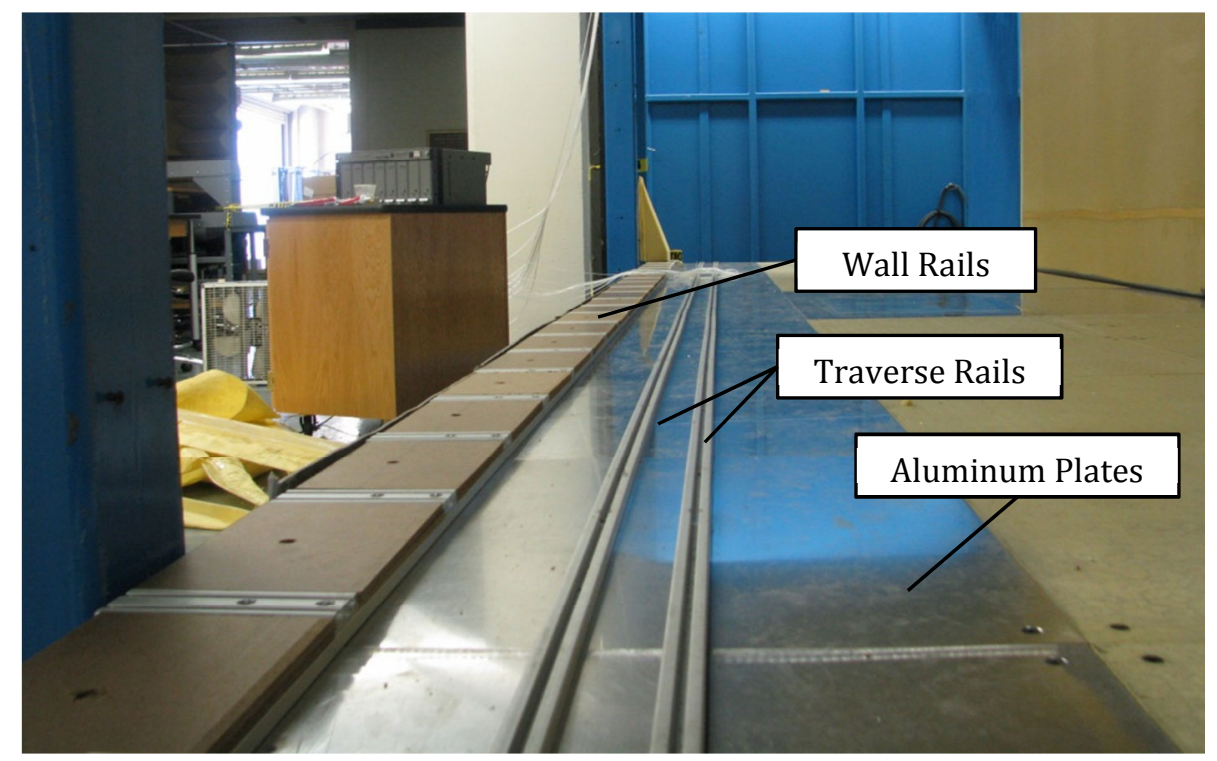

Figure 2.3. Rail system installed in anechoic chamber to mount false wall panels.

Each of the false wall panels was initially constructed out of a single sheet of $1.22 \mathrm{x}$ 1.78-m Lexan 13-mm thick. A slotted aluminum frame was then bonded to the back of the Lexan panels to provide additional structural stiffness and connection options. Eight right angle mounting brackets were then used to secure the false wall panels to the wall railings while still allowing the panels to be individually adjusted. Instrumentation slots were carefully milled into the Lexan frame in such a way as to ensure that there would be no protrusions into the flow while still maintaining a clean hydraulically smooth plate for the test conditions. When not in use, small $\left(<1-\mathrm{cm}^{2}\right)$ instrumentation slots or holes were taped over using packing tape less than $0.076-\mathrm{mm}$ thick, while larger slots were filled in with 'plugs' as described section 2.5.2. A typical frame configuration is depicted in Figure 2.4, with slight variations used to accommodate different types of instrumentation and their placements. 


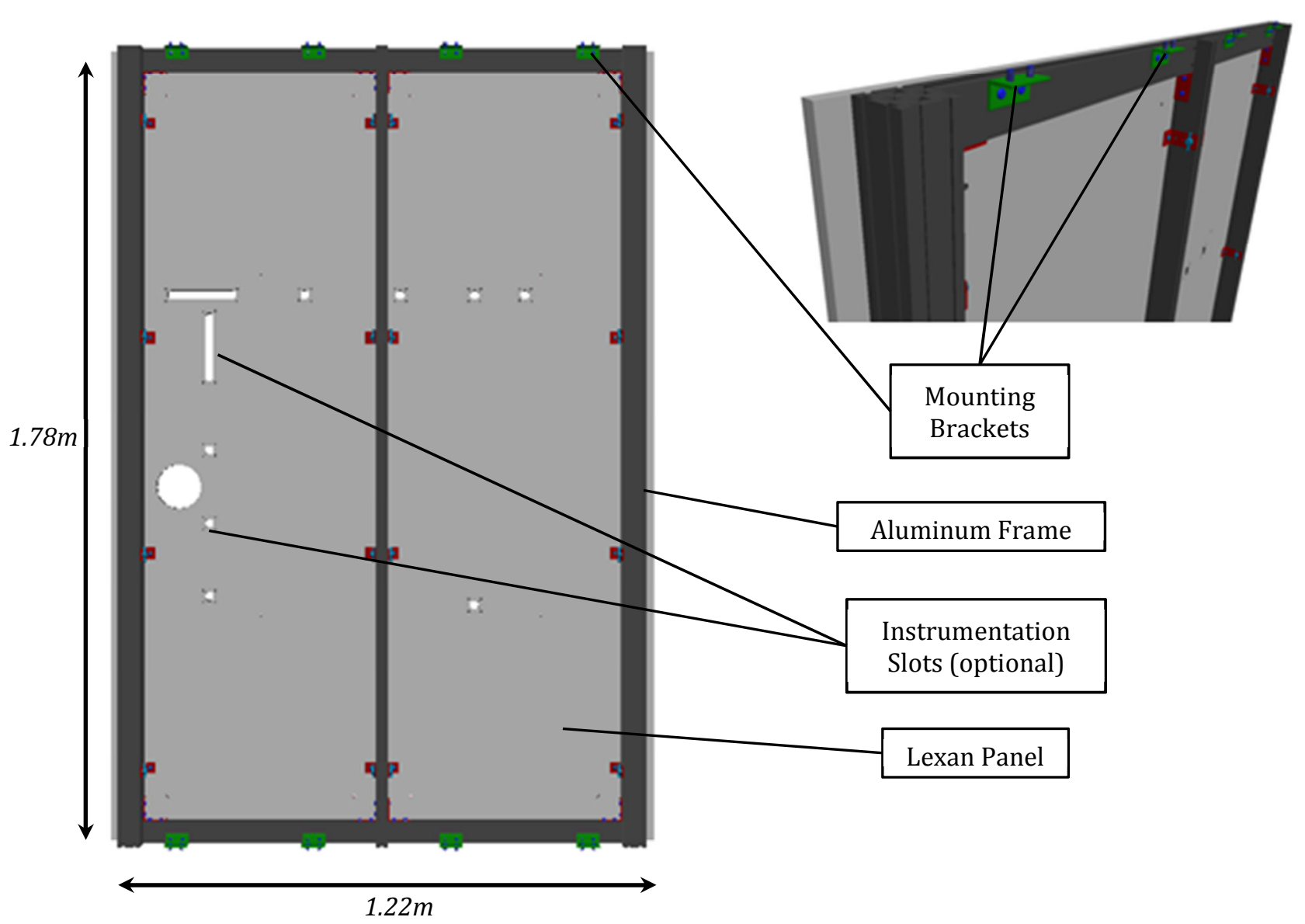

Figure 2.4. Typical false wall panel and frame schematic, non-flow side.

Once mounted along the railings in the anechoic test section, the false wall panels were aligned edge to edge keeping the panels flush with each other to within \pm 0.25 -mm with a streamwise gap of $3 \pm 3-\mathrm{mm}$. The panels were adjusted to obtain a zero-pressure gradient flow along the walls (see section 3.2) while maintaining these constraints. After the final panel adjustments, packing tape less than $0.076-\mathrm{mm}$ thick was used to cover the gaps between the panels and reduce any minor steps created by the panels not being completely flush. This configuration allowed the false wall panels to form an undisrupted $1.78 \times 7.32-m$ long surface on which this study was performed.

When installed, the leading edge of the false wall nominally sat $0.1-\mathrm{m}$ to the inside of the original test section boundary. To account for this, a curved fairing was built to be placed in the tunnel contraction and redirect the flow over the face of the false wall panels. This contraction fairing was constructed out of wooden ribbing overlaid with an ABS plastic skin. The modified contraction fairing reached $2.4-\mathrm{m}$ into the tunnel contraction and extended the contraction curve such that it finished $0.1-\mathrm{m}$ offset from the unmodified tunnel contraction. A panel method solution showed the modified geometry had no ill effects on the contraction's pressure gradient. A view of the modified contraction leading to the false wall testing surface is provided in Figure 2.5. 


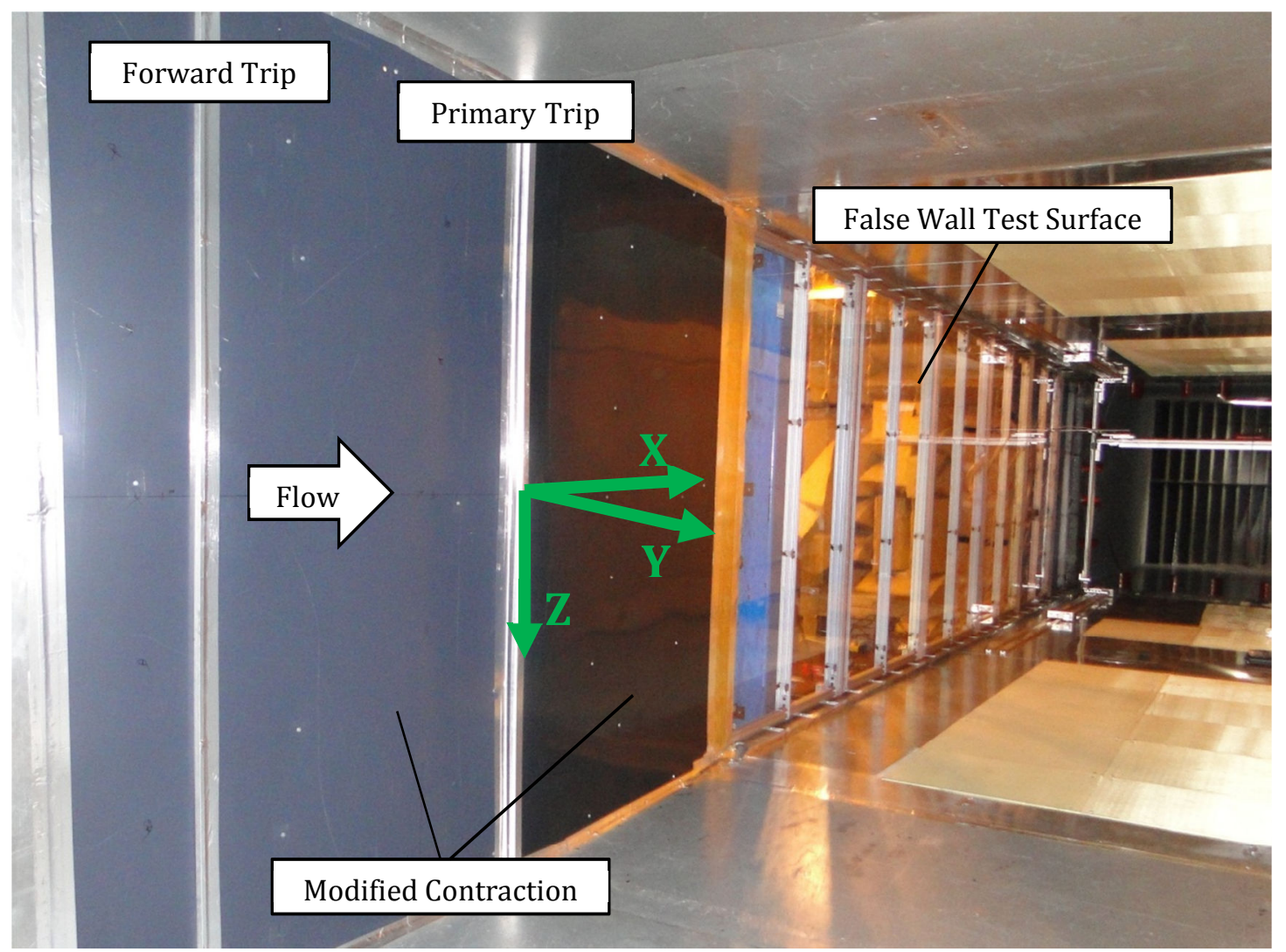

Figure 2.5. Modified contraction and false wall installed in the anechoic test section, smooth wall configuration.

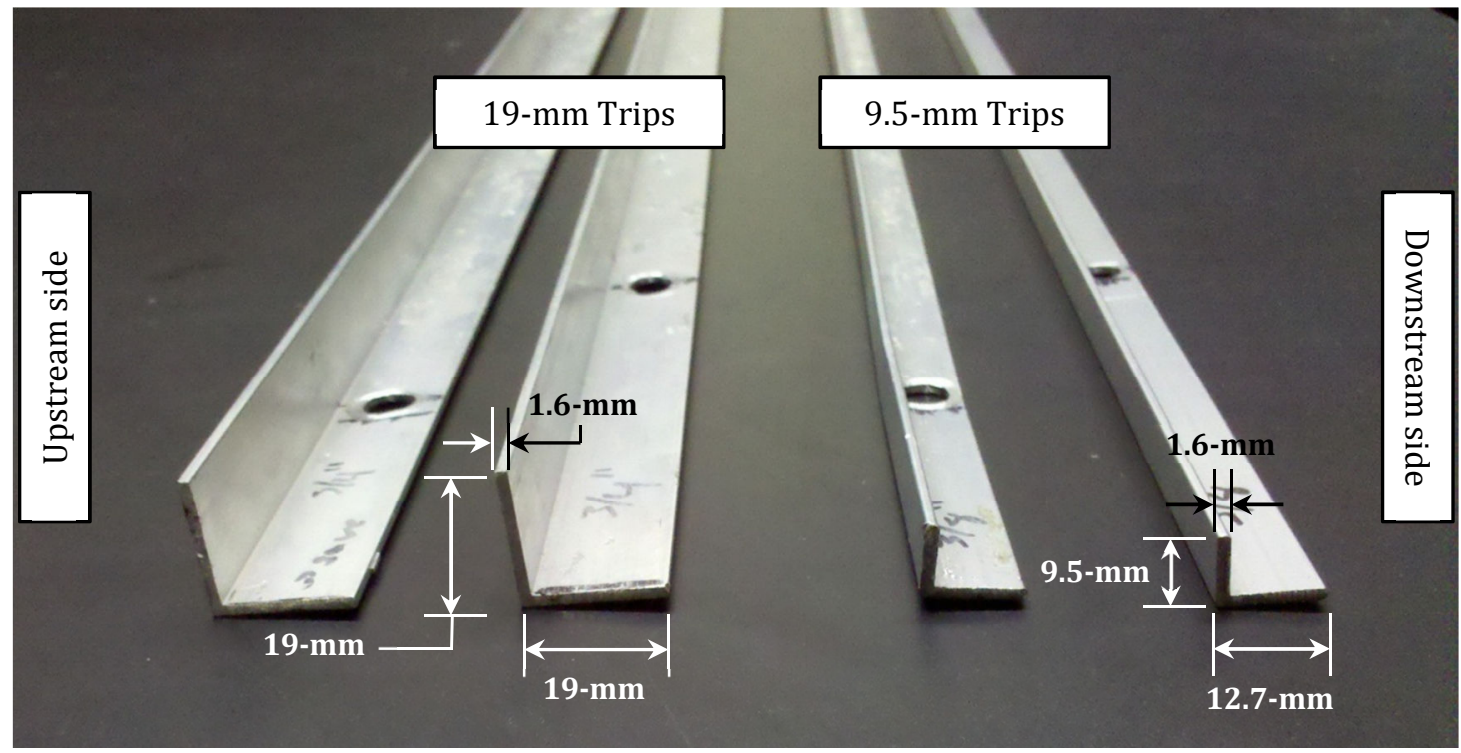

Figure 2.6. 19-mm and 9.5-mm trips.

Large boundary layer growth along the false wall was promoted through the use of 9.5-mm and 19-mm trips. Each trip consisted of a simple two-dimensional fence created from a strip of aluminum angle mounted with the flange on the downstream side as shown in Figure 2.6. The modified contraction fairing was built with two mounting hard points for 
the trips located 1.2-m and 2.1-m upstream of the leading edge of the false wall. This allowed for single or paired sets of trips to be installed and tested, with single trip configurations always being placed at the primary downstream trip mounting location. The configuration decided upon for wall pressure fluctuation measurements consisted of two of the 19-mm trips located both at the primary and forward trip locations. It was found that this trip configuration was capable of producing a boundary layer thickness in excess of $0.22-\mathrm{m}$ at $60-\mathrm{m} / \mathrm{s}, 7.0-\mathrm{m}$ downstream of the primary trip in the primary measurement location as seen in Figure 2.7. The primary measurement locations were located between 330 to 368 trip heights downstream of the primary trip. Previous theoretical studies (Gloerfelt 2010) had found flow irregularities due to a trip to have completely dissipated after 300 trip heights.

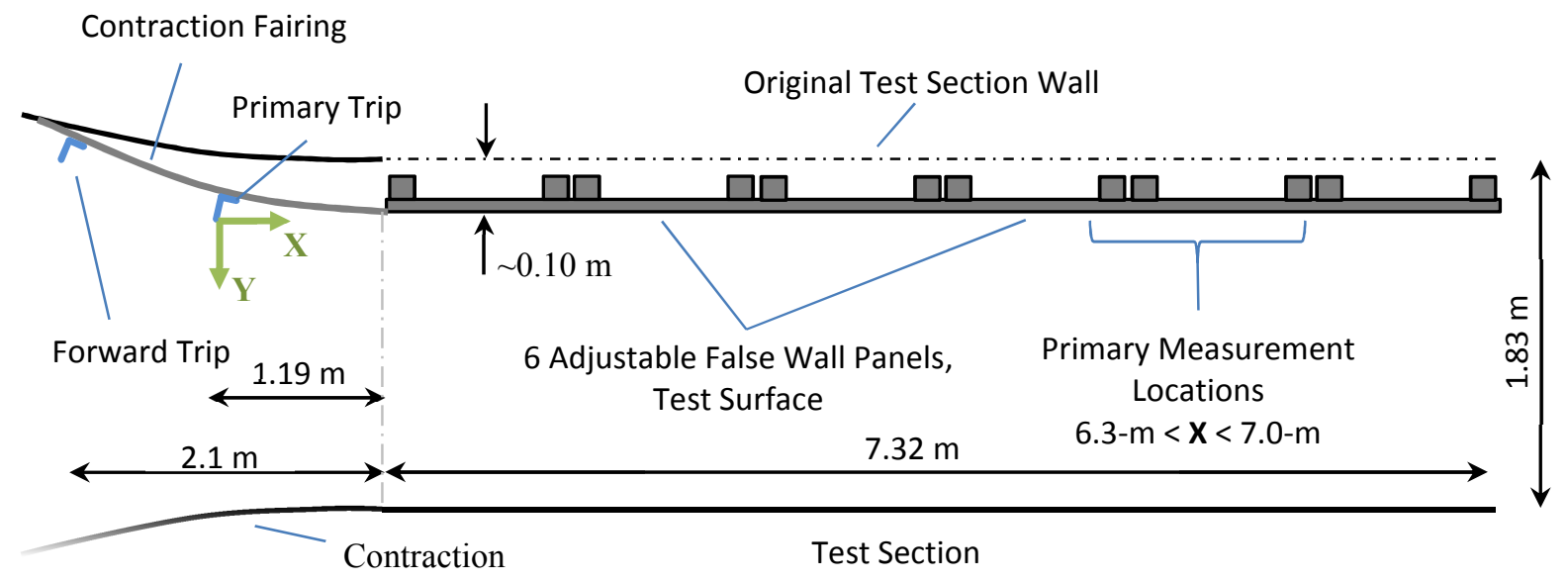

Figure 2.7. General tunnel setup diagram.

During testing the origin was defined in the $x$-direction relative to the primary trip location, with positive $x$ oriented downstream. The plane of the false wall was used as the $y$-axis origin, measured positive away from the wall. The $z$-axis was defined relative to the tunnel centerline, oriented positive downward, in order to obtain a right-handed coordinate system as seen in Figure 2.5 and Figure 2.7.

\subsection{Rough Surface}

Large discrete roughness patches consisting of 3-mm radius hemispheres spaced in a 16.5-mm square array were fabricated for use on the tunnel test surface. To form the roughness patches, a 3:1 ratio epoxy resin was evenly poured into a 1.83 x 1.24-m HDPE mold to form the roughness elements. Sheets of $0.46 \times 0.61-\mathrm{m}$ medium weight sketch paper were overlaid on top of the epoxy mold to form a smooth backing, with another layer of epoxy resin applied evenly on top of the paper. A layer of 30-weight Kevlar was then laid over the paper. All excess epoxy was removed and the fetch was evenly pressured while drying for 24-hours to ensure a uniform mold. After drying, the roughness fetch was removed from the molds and cut into $1.76 \times 1.22-\mathrm{m}$ patches to allow for instrumentation positioning while still maintaining continuity between fetches. Any missing or damaged 
roughness elements were repaired or replaced. If missing large amounts of roughness elements, the molds were discarded.

The Kevlar-paper roughness patches were then attached to 3 -mm thick $1.76 \times 1.22$ $\mathrm{m}$ aluminum plates using a Silicon sealant. The roughness fetches were centered vertically on the aluminum plates and staggered in the streamwise direction so that a $12.7-\mathrm{mm}$ of the roughness fetch would overhang the trailing edge of the aluminum plates, with an equivalently spaced section of aluminum exposed along the leading edge. The horizontal and leading edges of the roughness fetch were reinforced with superglue to ensure there would be no flow leakage between the roughness patch and the aluminum plating. 12 countersunk bolts were then used to attach each deterministically rough aluminum panel to the testing surface. The roughness patches were carefully aligned to ensure that no gaps, seams or dislocations would be present to the flow, with the trailing edge roughness fetch overhangs being aligned and used to cover the exposed leading edges on the subsequent aluminum panels. The result was a removable, uniformly deterministic rough surface spanning 1.76-m and stretching 7.32-m downstream, as can be seen in Figure 2.8.

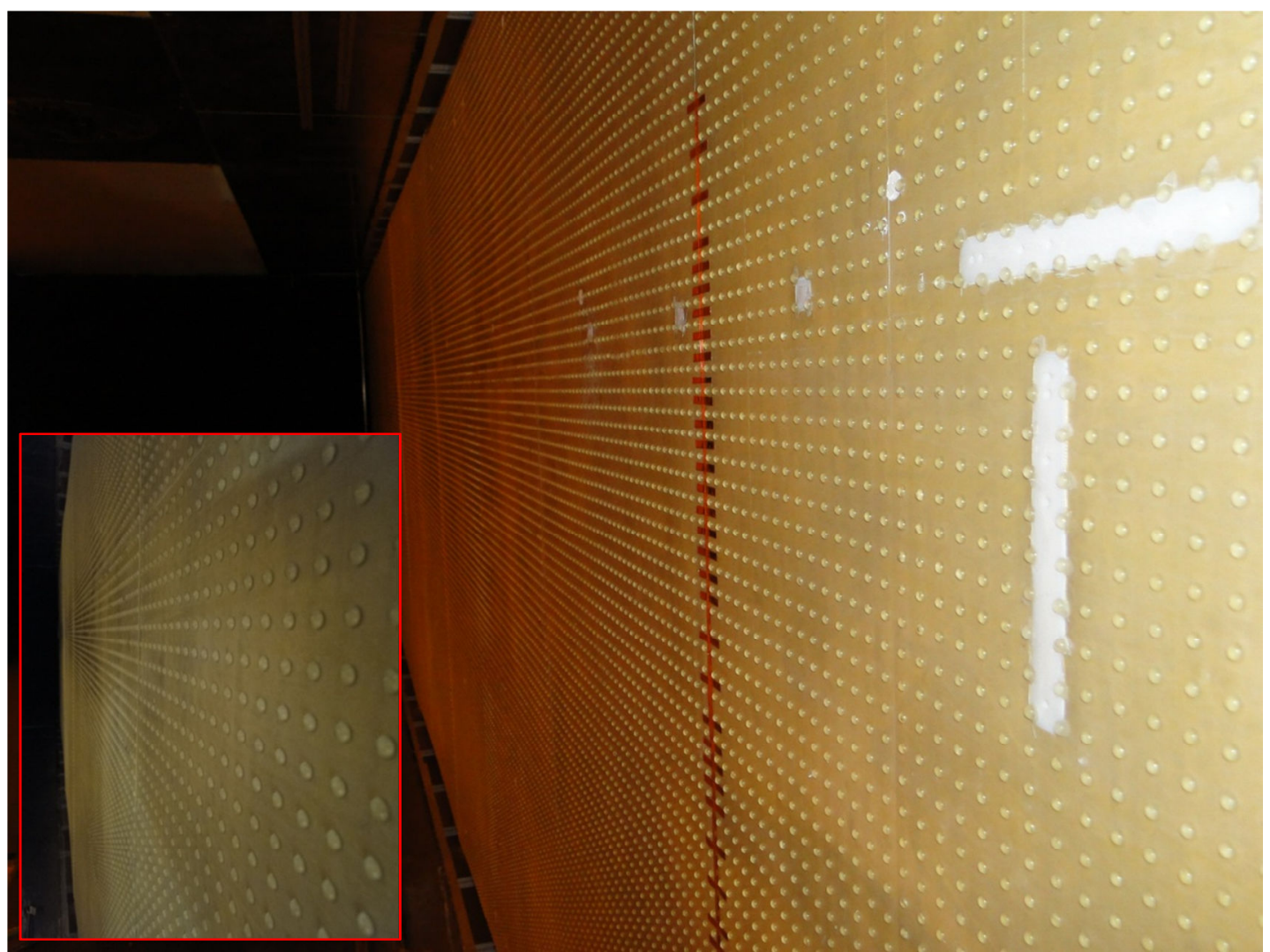

Figure 2.8. Installed 3-mm hemisphere deterministic rough surface. (Left inset) Hemisphere alignment at rough patch seams.

\subsection{Boundary Layer Velocity Instrumentation}

\subsubsection{Traverse System}

All boundary layer velocity instrumentation probes were mounted onto a traverse capable of automated three-axis movements as controlled by a laptop computer. Traverse 
movement was controllable down to a $0.025-\mathrm{mm}$ resolution. When installed, the traverse system consisted of two floor and two ceiling streamwise railings, two vertical rails, and a single horizontal railing as seen in Figure 2.9. A 0.98-m sting used to hold velocity instrumentation probes was mounted on the horizontal railing shown in Figure 2.10. A vibration damping device was utilized to minimize vertical sting vibrations at high flow speeds. Note that the upstream $0.32-\mathrm{m}$ spanwise sting extension and $0.27-\mathrm{m}$ streamwise extension pictured in Figure 2.10 were only used for the single and four sensor hotwire anemometers; the flat head Pitot was directly mounted to the sting with its own $0.27-\mathrm{m}$ spanwise extension towards the wall. When installed, the full traverse system created approximately an $11 \%$ tunnel area blockage resulting in higher fan speeds being run to achieve similar test section flow velocities.

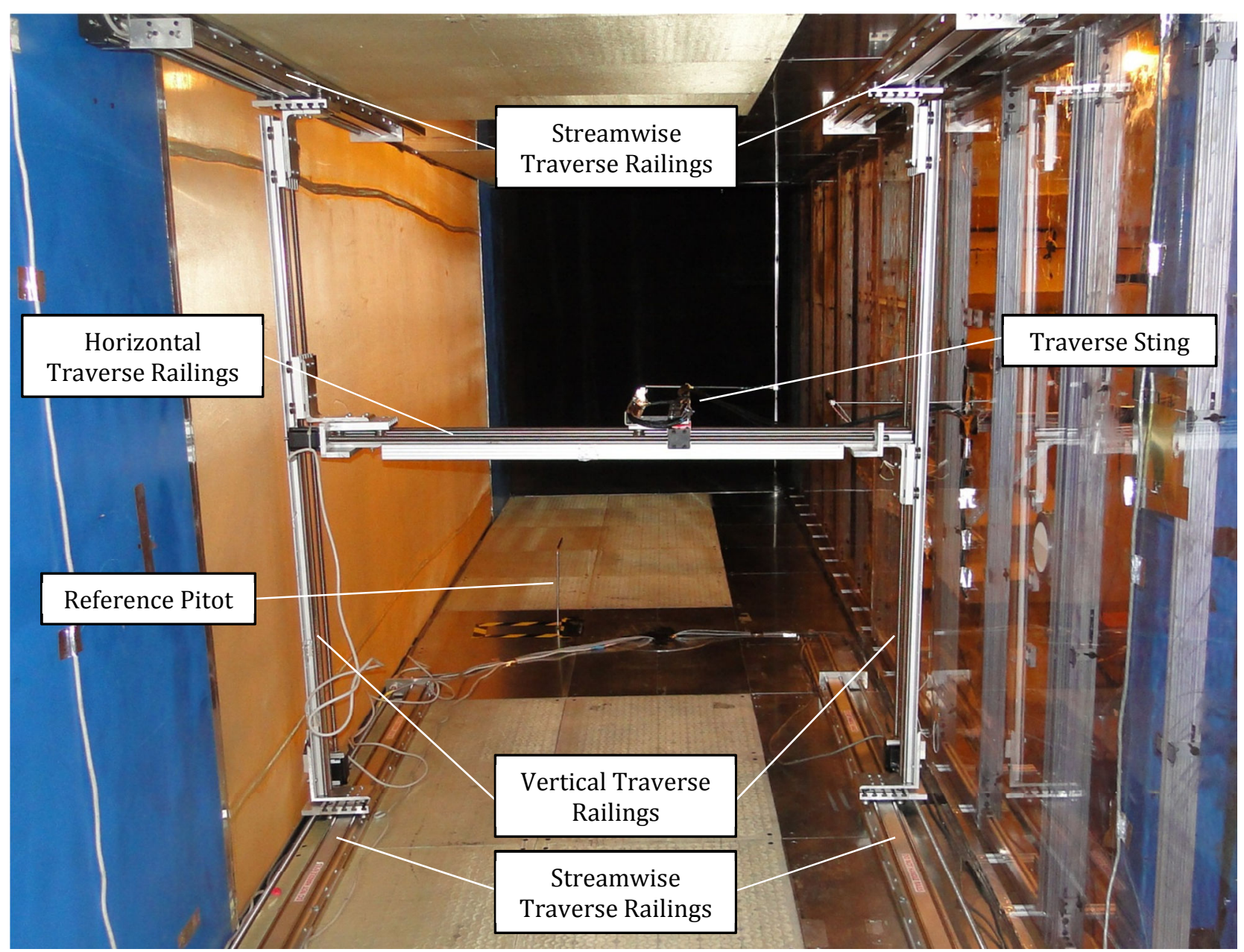

Figure 2.9. Stability Wind Tunnel Traverse system. Photograph taken by Mike Morton (2011), used with permission. 


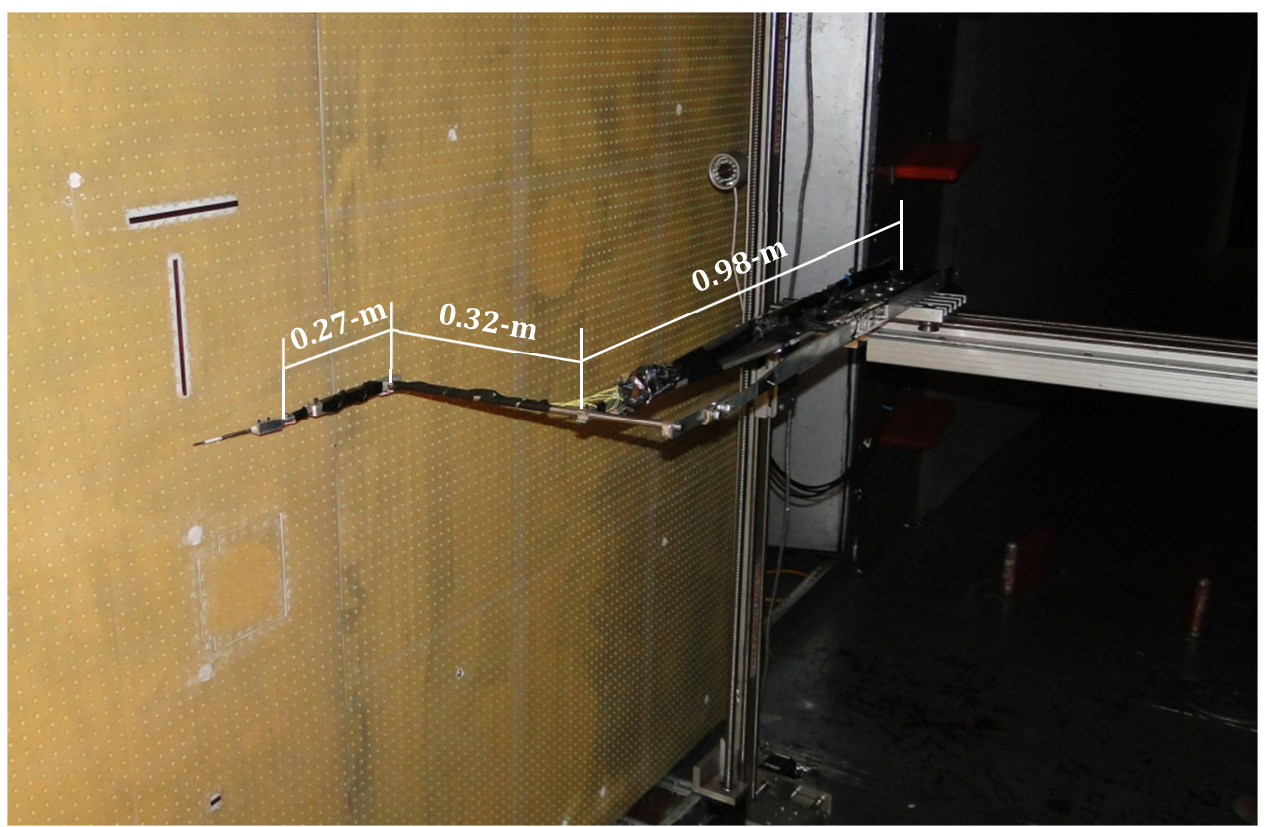

Figure 2.10. Traverse system sting and probe mount.

\subsubsection{Flat Head Pitot Instrumentation}

Mean velocity boundary layer profiles were taken using a flat-head Pitot with measurements spanning from the freestream down to $0.25-\mathrm{mm}$ away from the wall. The mouth of the Pitot had an opening $1 \times 0.3-\mathrm{mm}$ in the directions parallel and perpendicular to the wall, respectively. Static pressure was obtained using the pressure ports located on the floor and ceiling of the tunnel as described in section 2.5.1, with the floor port nearest the measurement location being used as the local freestream static pressure. Data was recorded using a Setra pressure transducer capable of measuring pressures from $\pm 15^{\prime \prime} \mathrm{H}_{2} \mathrm{O}$. The signal output was recorded using a 64 channel 16-bit Agilent E1432 digitizer and signal analyzer. Flat head Pitot measurements were taken $4.7-\mathrm{m}, 5.0-\mathrm{m}, 5.4-\mathrm{m}, 7.0-\mathrm{m}$ and 7.6-m downstream of the trip to measure boundary layer growth along the smooth wall test surface. Additionally, at $4.7-\mathrm{m}$ and $7.0-\mathrm{m}$ downstream of the trip, the twodimensionality of the boundary layer was checked by taking profiles at 0 - $\mathrm{m}$ and $\pm 0.46-\mathrm{m}$ from the tunnel centerline. While taking boundary layer profiles over the rough surface, the flat head Pitot tip was aligned between the roughness elements in the center of the roughness array as shown in Figure 2.11. 


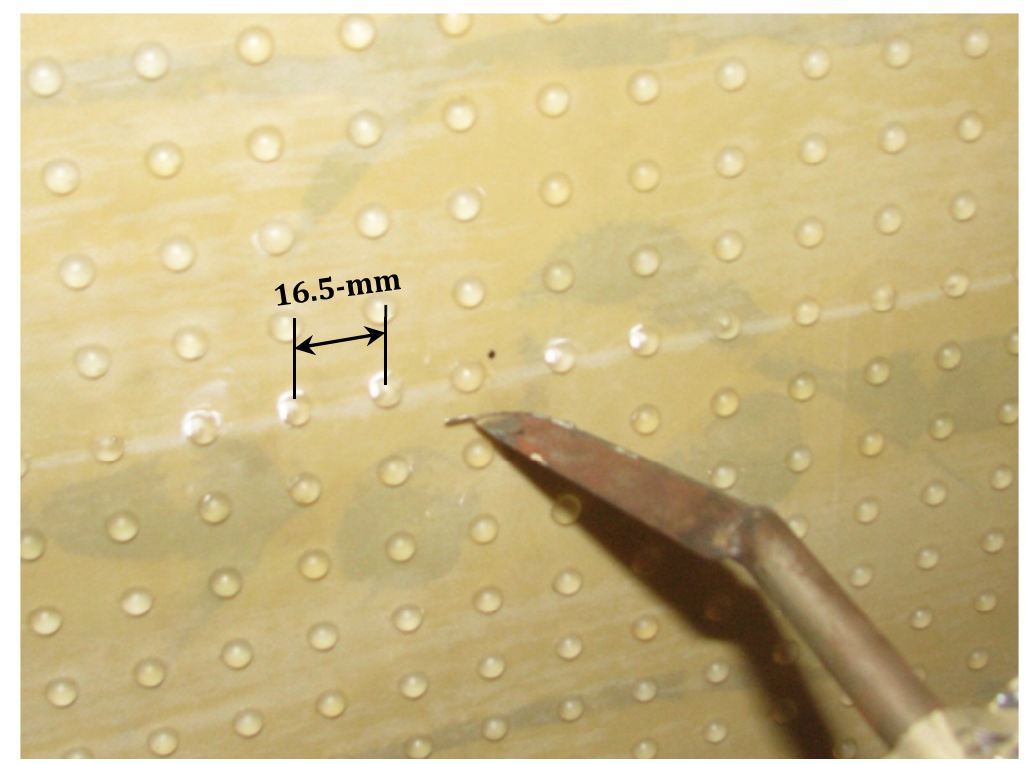

Figure 2.11. Flat head pitot.

\subsubsection{Hotwire Anemometer}

Boundary layer velocity fluctuation measurements were taken using both single and four sensor hotwire anemometer probes. All probes were mounted on the traverse system sting shown in Figure 2.10 facing parallel into the flow to within $\pm 4^{\circ}$, with the exception of the quad-wire mount when taking measurements over the rough wall. While taking measurements over the rough wall, the probe mount was angled at a $12.1^{\circ}$ angle of attack yawed toward the test surface. While small angles could easily be accounted for through a post analysis probe rotation, the larger angles of attack saw reduced Reynolds stress tensors believed to be due to calibration sensitivities that had to be accounted for as described in section 2.4.4. Quad-wire measurements in the smooth wall configuration were not affected.

The four-sensor hotwire, or quad-wire system, shown in Figure 2.12 consisted of two perpendicular pairs of $x$-wires positioned at the probe tip to form a $0.75-\mathrm{mm}^{3}$ measurement volume capable of measuring the three-components of the flow velocity. Each quad-wire system was initially calibrated in an isolated jet stream of known velocity. To start the initial calibrations, the probe was aligned parallel with the flow and the velocity was varied. King's Law was then used to relate probe voltage output to flow velocity measurements. Next the quad-wire probes were pitched and yawed by up to $45^{\circ}$ to obtain a probe angle calibration, which was applied through the use of a look up table method as described in Wittmer et al. (1998). During testing, a probe velocity calibration was taken between each data set, however only the initial angle calibration was performed. 


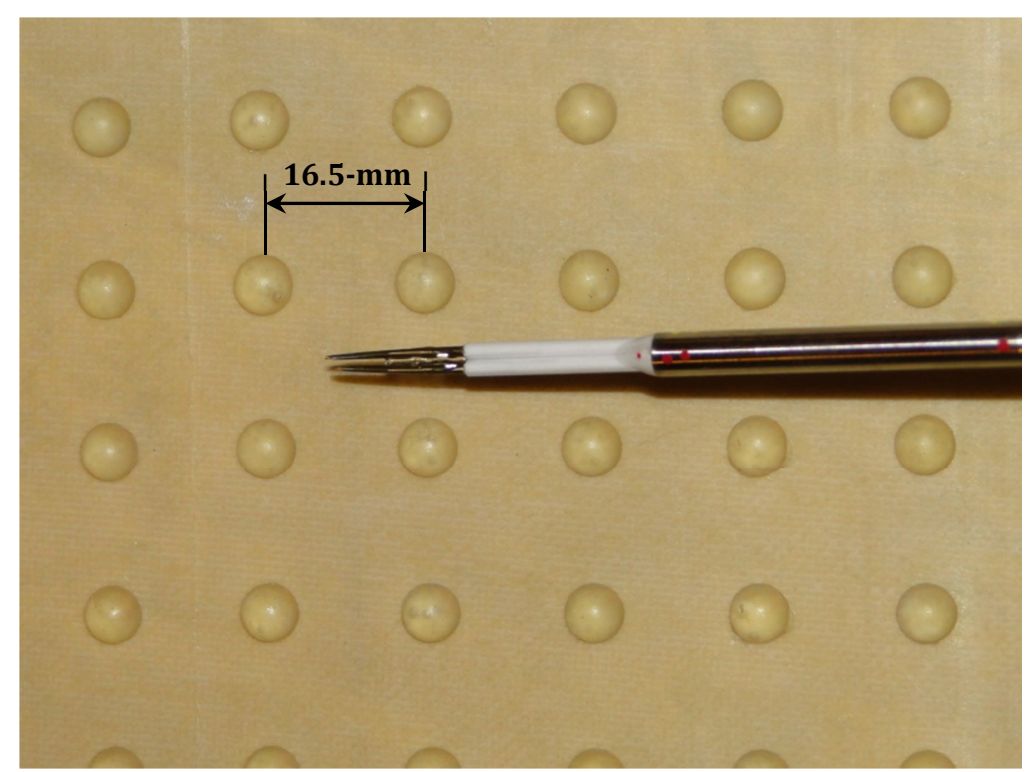

Figure 2.12. Four component hotwire anemometer (Quad-wire).

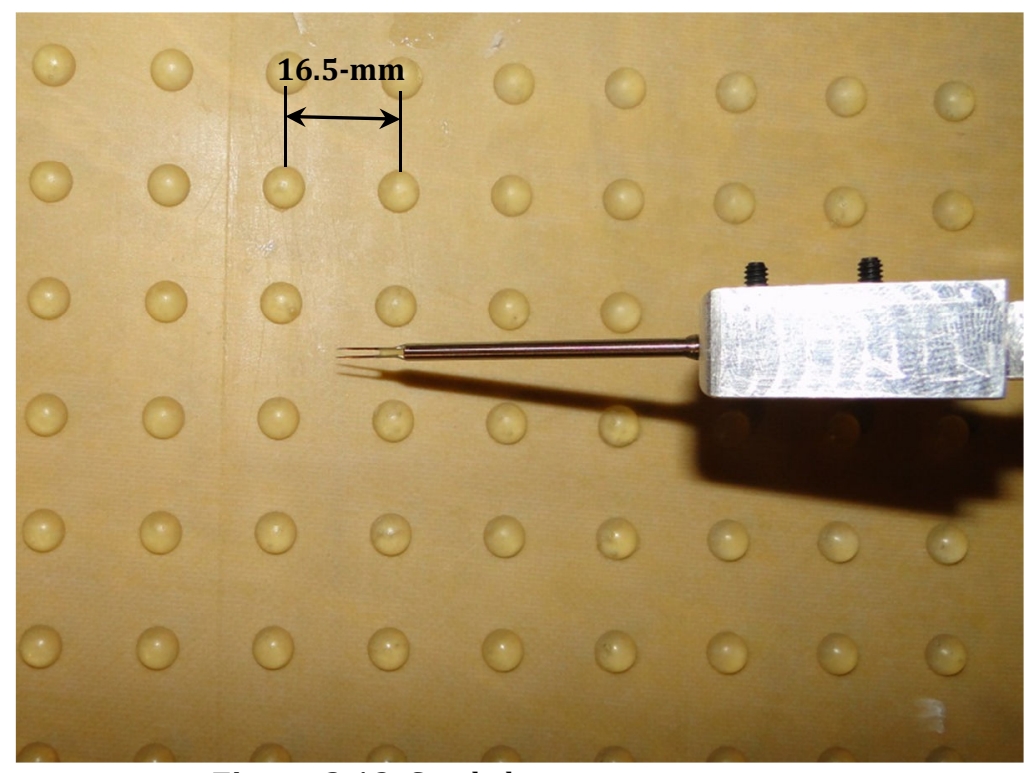

Figure 2.13. Single hotwire anemometer.

The single hotwire system as shown in Figure 2.13 required only a velocity calibration. This calibration was performed in the wind tunnel free stream between every other data set taken during testing. King's Law was again used to relate probe voltage output to flow velocity measurements. Both single and quad-wire systems were operated by a Dantec Dynamics StreamLine Constant Temperature Anemometry (CTA) System which was capable of automatically optimizing the sensor signal during measurements. A flat frequency response below $18-\mathrm{kHz}$ was observed while using this system with either quad-wire or single hotwire probes. The reference transducer measuring the pressure from the reference pitot shown in Figure 2.9, as well as the hotwire anemometer signals were taken and recorded using a 64-channel, 16-bit Agilent E1432 digitizer and signal 
analyzer. The Agilent digitizer was controlled by a laptop computer and adjusted for the ambient temperature of the boundary layer flow.

The three-component boundary layer profiles were taken using the quad-wire system $4.7-\mathrm{m}$, and 7.0-m downstream of the trip. Quad-wire measurements were taken from the freestream down to 4-mm away from the wall. It was shown by Wittmer et al. (1998) that measurements in a similarly scaled turbulent pipe flow were free of gradient errors down to 3-mm from the wall. Single-wire measurements were taken 4.7-m and 7.0$\mathrm{m}$ downstream of the trip between the freestream and a $0.5-\mathrm{mm}$ distance away from the wall. In both cases, in the presence of roughness, the probe ends were aligned to the center of the roughness array, between roughness elements.

\subsubsection{Quad-wire Angle Transformation}

Quad-wire probe measurements taken at yaw angles of greater than $8^{\circ}$ were later found to have calibration issues affecting mainly the Reynolds stress tensors that were not fully corrected when applying a post measurement probe angle rotation. A concurrent study (Morton and Devenport 2011) using the same wind tunnel and smooth false wall configuration had used the same quad-wire setup and moving traverse as found in the present study for rough surfaces, only with the addition of a second quad-wire probe mounted on a single-axis traverse. This second quad-wire probe was used to take simultaneous measurements at a probe yaw angle of less than $4^{\circ}$. During these two-point boundary layer measurements, up to 12 data points were taken at congruent positions and conditions using both the high and low angled probes.

Due to the matching conditions between moving and single-axis probes, boundary layer profile measurements could be expected to match between the two sets. While mean velocity profiles were found to be consistent between the different probes, Reynolds stress profiles were not found to be in complete agreement, similar to the circumstances found by Borgoltz (2007). The differences in Reynolds stress measurements throughout the boundary layer profile can be seen in Figure 2.14. 

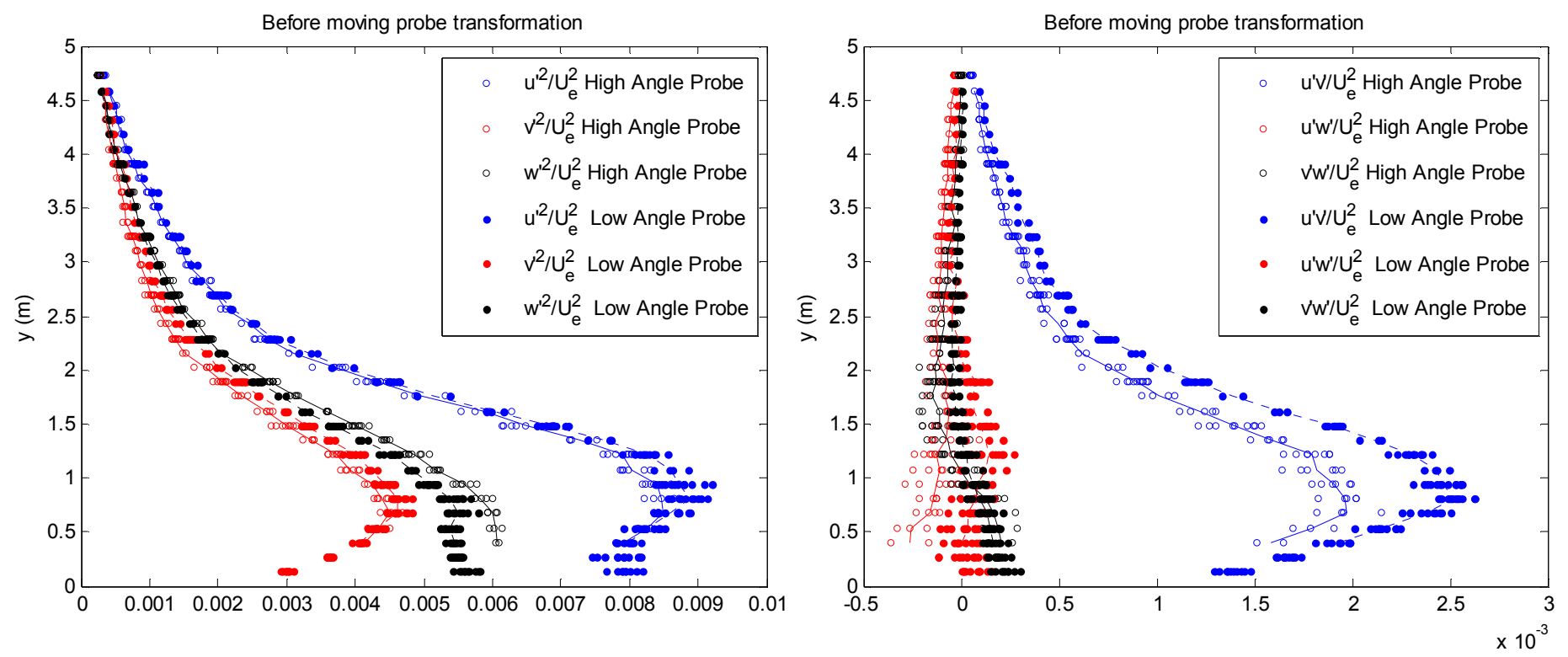

Figure 2.14. Boundary layer turbulence data for high and low-angled probes, untransformed, measured at same conditions.

The single-axis quad-wire probe profiles taken using low yaw angles and positioned nearly parallel to the flow were taken to be correct. It was also assumed that since the flows were largely unidirectional, typically only a single point was used from the probe angle calibration. This assumption thus implied that because the mean velocities were consistent across the different probe angles, errors in the local slope of the notional calibration surface for the high angle probe were the cause of the differences in the Reynolds stress profiles.

$$
\left\{\begin{array}{c}
u_{\text {trans }}^{\prime} \\
v_{\text {trans }}^{\prime} \\
w_{\text {trans }}^{\prime}
\end{array}\right\}=\left[\begin{array}{lll}
T_{11} & T_{12} & T_{13} \\
T_{21} & T_{22} & T_{23} \\
T_{31} & T_{32} & T_{33}
\end{array}\right]\left\{\begin{array}{c}
u^{\prime} \\
v^{\prime} \\
w^{\prime}
\end{array}\right\}
$$

One could therefore perform a transformation on the high angled probe to correct it to match the low angled single-axis probe. Equation 2.1 relates the corrected or 'transformed' velocity fluctuations $u_{\text {trans }}^{\prime}, v_{\text {trans }}^{\prime}$ and $w_{\text {trans }}^{\prime}$ to the initial velocity fluctuation values through the transformation matrix $\boldsymbol{T}$. In the study where both low and high angled probes were present, $\boldsymbol{T}$ could be determined by minimizing the differences between the low and high angled probe measurements as depicted in Borgoltz (2007). The results after a transformation was applied can be seen in Figure 2.15. 

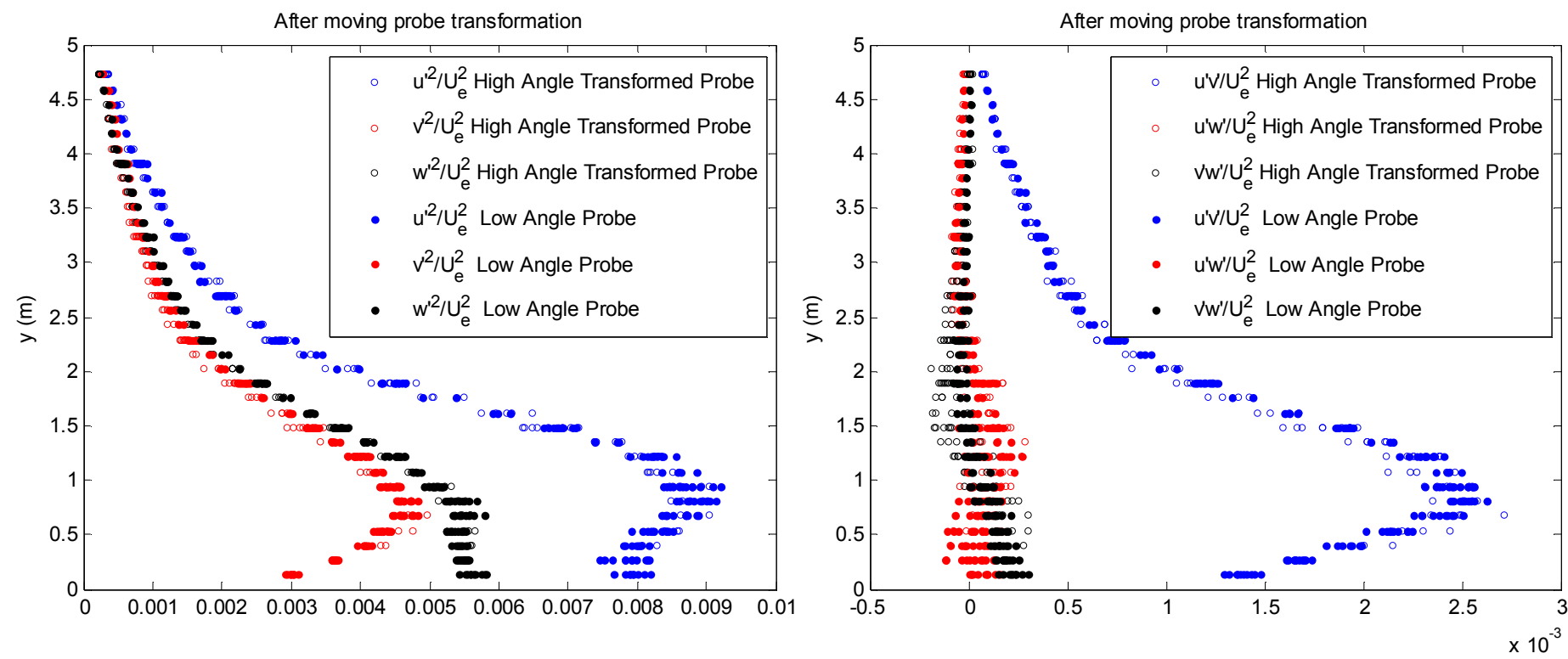

Figure 2.15. Boundary layer turbulence data for high and low-angled probes, transformed, measured at same conditions.

In the present study, a congruent low-angled probe was not present while taking measurements over rough surfaces. However, the same probe and probe calibration used in the previous study at high angles was again being used in a similar manner at a similar probe yaw angle (12.1 instead $11.4-11.5^{\circ}$ from the previous study). Additionally, it was known that for a probe aligned parallel to the flow with a $0^{\circ}$ yaw angle, the transformation matrix would simply be an identity matrix. Thus in order to estimate the required transformation matrix for the quad-wire probe over the rough surface in the present study, a linear fit based on yaw angle from the known data with a low angle probe was determined for each component of the transformation matrix $\boldsymbol{T}$ as seen in Figure 2.16. 

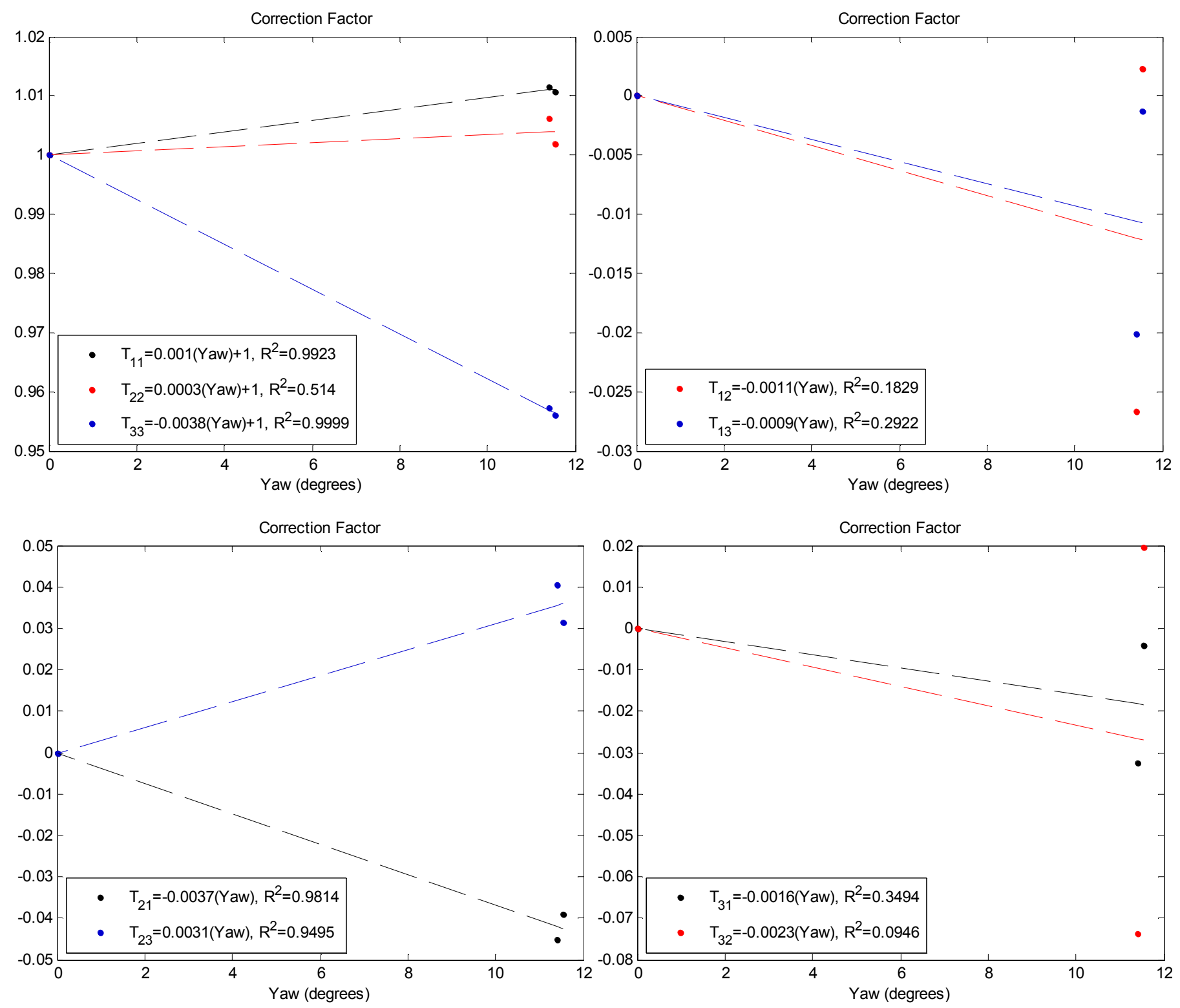

Figure 2.16. Quad-wire high angle probe correction transformation factor linear estimations.

Transformation matrix components where the linear regression coefficient of determination was found as $R^{2}<0.5$ were deemed to be unaffected by the yaw angle of the probe, with variances stemming only from the probe calibration uncertainties. Therefore, for the transformation estimation these elements were estimated as their equivalent identity matrix components. The final transformation matrix for the quad-wire probe yawed at $12.1^{\circ}$ was therefore found as Equation 2.2 with the transformation applied as described in Equation 2.1.

$$
\boldsymbol{T}\left(12.1^{\circ}\right)=\left[\begin{array}{ccc}
1.0117 & 0 & 0 \\
-0.0445 & 1.0042 & 0.0378 \\
0 & 0 & 0.9543
\end{array}\right]
$$




\subsection{Surface Pressure Instrumentation}

\subsubsection{Pressure Tap Instrumentation}

Mean wall pressure was found using twenty four $1 / 2-\mathrm{mm}$ pressure taps located along the wall. These pressure taps were installed on the floor and ceiling of the test section rather than on the test surface itself. This ensured the taps would be unobstructed and usable for both smooth and rough wall configurations. The ports were located between $0.25-\mathrm{m}$ and $0.36-\mathrm{m}$ away from the test surface depending on wall position and used the assumption that the mean static pressure would remain constant inside the boundary layer. Mean pressure data was recorded using an Esterline NetScanner Model 98RK pressure scanner. This data was used to adjust the positioning of the walls and minimize the wall pressure gradient. Additionally, this data was taken throughout the tests in conjunction with other instrumentation to ensure the wall pressure gradient remained minimized.

\subsubsection{Wall Pressure Microphone Instrumentation}

Wall pressure fluctuation measurements were made using six Brüel \& Kjaer (B\&K) type 4138-A-015 1/8-in microphones ${ }^{1}$. The sensing area of five of these microphones was reduced by fitting them with $1 / 2$-mm pinhole caps. Previous measurements under a wall jet boundary layer, by Devenport et al. (2011) showed a $1 / 2-\mathrm{mm}$ pinhole to be sufficiently small enough to resolve pressure fluctuations up to $20 \mathrm{-kHz}$. However, Gravante et al. (1998) had a more conservative estimate on the pinhole size required to ensure sensor attenuation was not found at high frequencies. In this paper, it was suggested that the dimensionless pinhole diameter $d^{+}=d u_{t} / v$, where $v$ is the kinematic viscosity and $u_{t}$ is the friction velocity, should be smaller than 18 to ensure no sensor attenuation occurred for frequencies up to $\omega v / u_{t}^{2}=1$.

In Table 2.1, the boundary layer length scales $u_{t} / v$ and the dimensionless pinhole diameters $d^{+}$are shown for the present study. The non-bolded values represented in this table are interpolated for the pressure fluctuation flow conditions as is explained in section 3.3.1, equivalent to what is done in Table 3.2. Table 2.1 shows that the pinhole diameters used in this study do not quite meet the requirements set by Gravante et al. It should be noted, however, that the instrumentation limitations set the Nyquist frequency in the present study to only $26-\mathrm{kHz}$, corresponding to normalized frequencies of $\omega v / u_{t}^{2}=0.62$ at $67.2-\mathrm{m} / \mathrm{s}$ over smooth walls and only $\omega v / u_{t}^{2}=0.31$ at $60-\mathrm{m} / \mathrm{s}$ over rough walls as indicated by the $\left(\omega v / u_{t}^{2}\right)_{N y q}$ entry. This would suggest that the dimensionless pinhole diameter requirements would not need to be as stringent for the higher flow velocity conditions where $\left(\omega v / u_{t}^{2}\right)_{N y q}<1$. Thus while sensor attenuation is not expected to be as severe as first inspection of the dimensionless diameter values for the higher flow speed conditions would suggest, it could still be expected to affect the microphone measurements along the highest frequencies to some extent.

\footnotetext{
1 Early surface pressure fluctuation work attempted to use 48 Sennheiser KE-4-211-2 electret condenser microphones fitted with $1 / 2-\mathrm{mm}$ brass pinhole caps. The microphones were aligned in 2 parallel streamwise and one spanwise arrays with similar spacing as that used by the B\&K microphones. However, after analyzing early measurements taken using this system, it was found that the Sennheiser microphones may have been going over range at high speeds and did not provide the low frequency fidelity that could be offered by the less available, but more sensitive B\&K type 4138 microphones.
} 
Table 2.1. Flow length scales and dimensionless pinhole size for $0.5-\mathrm{mm}$ pinholes under testing conditions.

\begin{tabular}{ccccc} 
Speed $(\mathbf{m} / \mathbf{s})$ & $\mathbf{d}^{+}$ & $\mathbf{v} / \mathbf{u}_{\mathbf{t}}(\boldsymbol{\mu m})$ & $\left(\boldsymbol{w v} / \mathbf{u}_{\mathbf{t}}\right)_{\text {Nyq }}$ & Configuration \\
\hline 22.4 & 24.1 & 20.7 & 4.65 & Smooth \\
33.6 & 33.9 & 14.7 & 2.13 & Smooth \\
44.8 & 43.3 & 11.5 & 1.25 & Smooth \\
56 & 52.3 & 9.6 & 0.84 & Smooth \\
67.2 & 60.9 & 8.2 & 0.62 & Smooth \\
20 & 33.4 & 15.0 & 2.49 & 3-mm Rough \\
$\mathbf{3 0}$ & $\mathbf{4 7 . 2}$ & $\mathbf{1 0 . 6}$ & $\mathbf{1 . 1 4}$ & 3-mm Rough \\
40 & 60.3 & 8.3 & 0.66 & 3-mm Rough \\
50 & 72.7 & 6.9 & 0.43 & 3-mm Rough \\
$\mathbf{6 0}$ & $\mathbf{8 4 . 5}$ & $\mathbf{5 . 9}$ & $\mathbf{0 . 3 1}$ & 3-mm Rough
\end{tabular}

Prior to testing, the pinhole microphones were calibrated as described in section 2.5.3 below. The sixth microphone was used as a reference and maintained its unaltered form with the standard factory installed protective cap used to sense the low-frequency acoustic background of the wind tunnel. Back ground acoustic noise was later removed from the signals of the measurement microphones, with details of the acoustic cancellation methods being described in section 2.6.1. During testing, data from all microphones was collected though a B\&K type 3050-A-060 Pulse Analyzer sampling at $65536-\mathrm{Hz}$ for 32 seconds. The amplitude gain of the microphones was calibrated daily using a B\&K type 4228 Pistonphone operating at $250-\mathrm{Hz}$. The Pulse system was run using a $22.4-\mathrm{Hz}$ filter, which was accounted for as detailed in section 2.5.4.

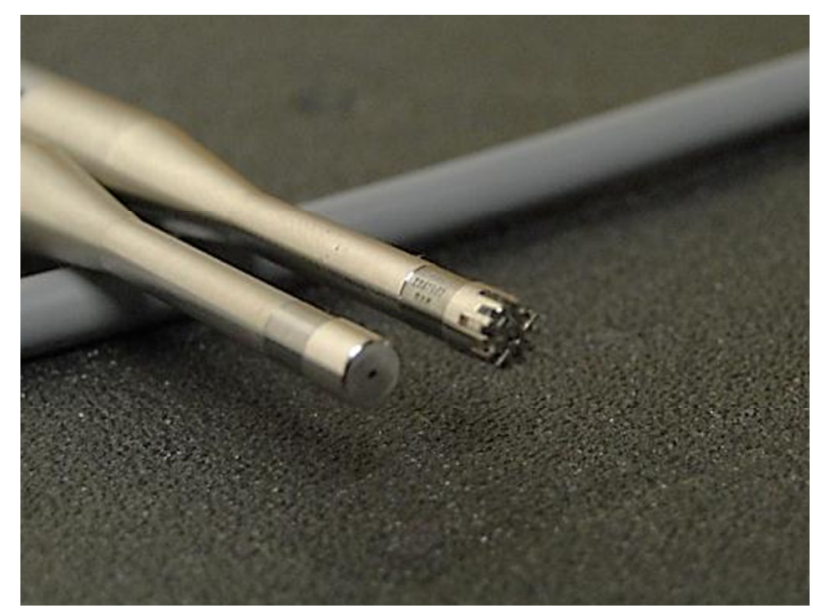

Figure 2.17. B\&K Type 4138-A-15 microphones with $1 / 2-\mathrm{mm}$ pinhole cap (left) and standard protective cap (right).

To place the microphones in the wall, each was fitted into two types of plastic sleeves created by an Objet Alaris 30 3D printer, with each type of sleeve holding either a single microphone or up to five microphones each. The plastic sleeves were designed to keep the microphone heads flush with the front face of the sleeve to within $\pm 0.02-\mathrm{mm}$. Slots 
were cut into the Lexan test surface allowing the sleeves with included microphones to be inserted from the back, leaving the microphone sensor head flush with the flow side of the smooth test surface to within \pm 0.2 -mm as can be seen in Figure 2.18. Unused slots in the false wall were filled with plastic plugs created on the 3D printer.

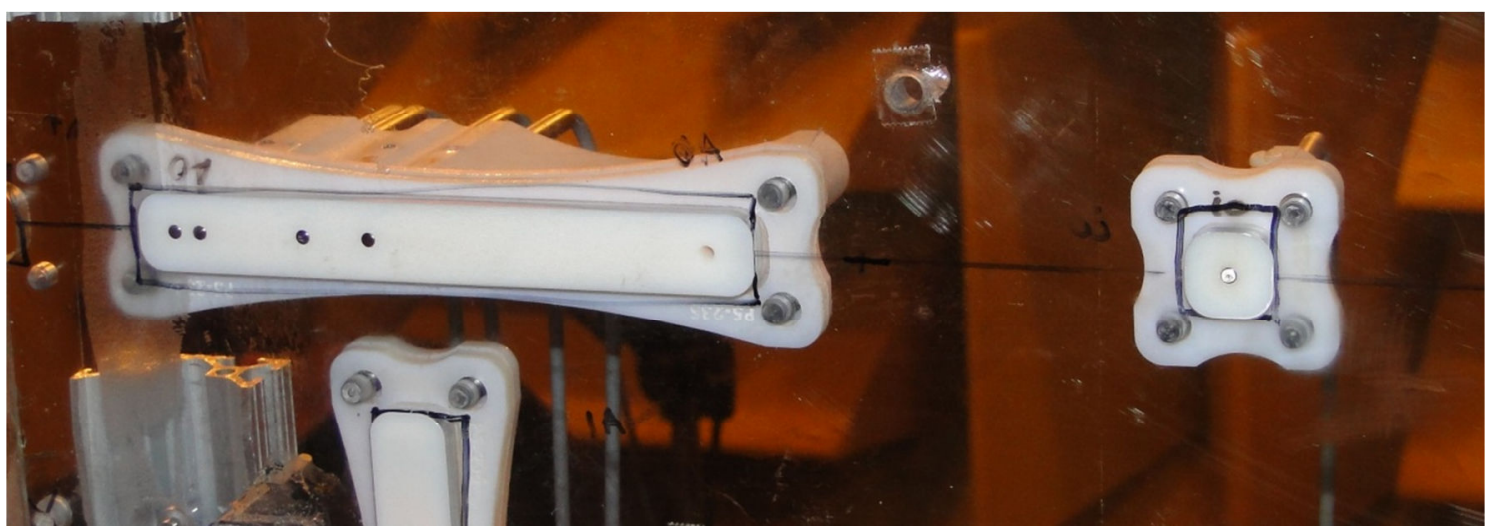

Figure 2.18. Two microphone sleeve types used, embedded in the clean surface test plate. Photograph taken by Manuj Awasthi (2011), used with permission.

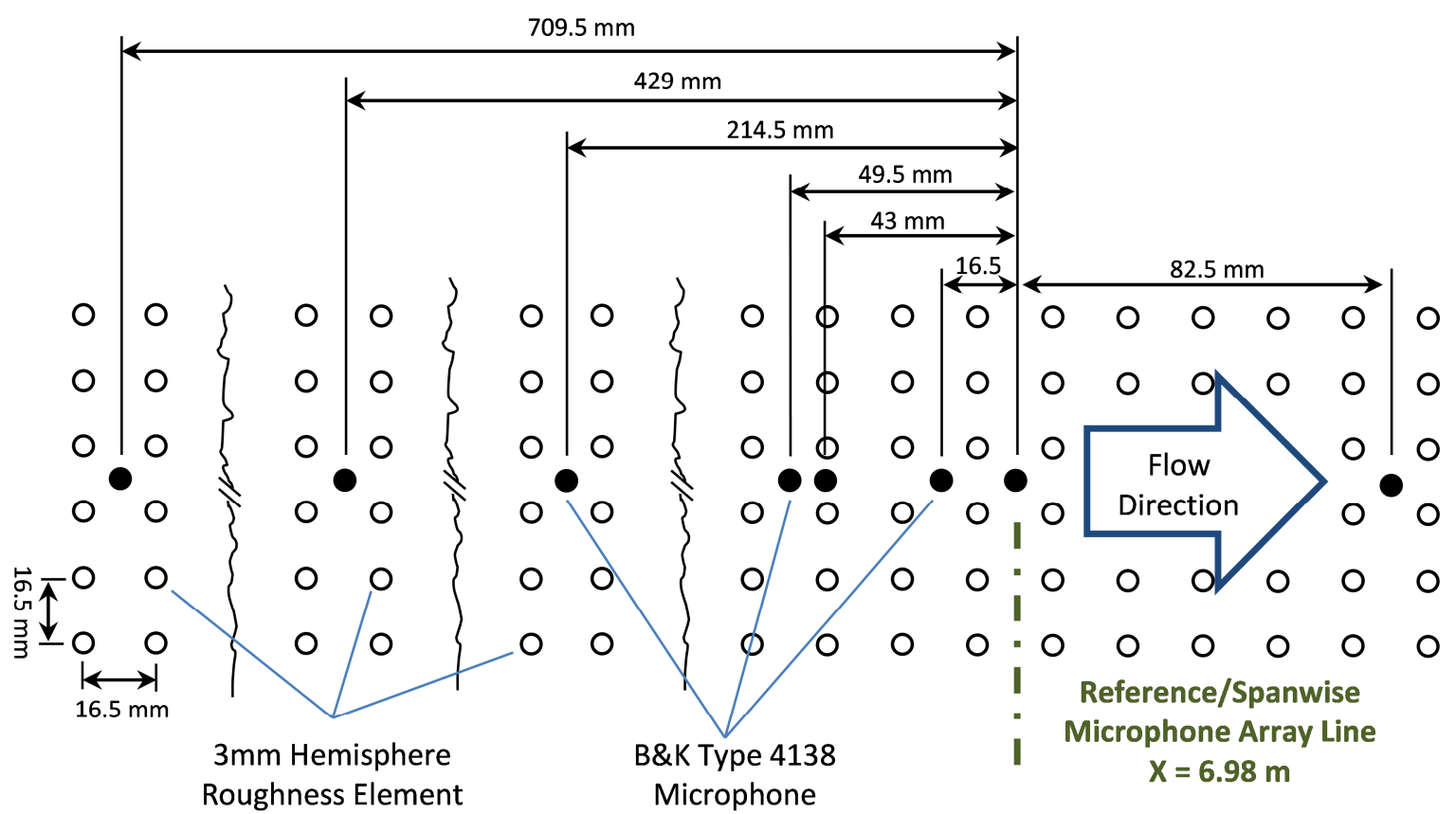

Figure 2.19. Streamwise microphone placement diagram. Relative positioning of the microphones on the spanwise array is equivalent with a $90^{\circ}$ rotation.

When placed in the roughness, the microphones and plastic sleeves were aligned flush with the top surface of the roughness substrate. Single roughness elements were glued to the sleeves where necessary as to ensure an uninterrupted pattern. Microphone spacing was unchanged from the smooth wall configuration. The roughness fetch was positioned such that all but two of the microphones, including one spanwise and one streamwise microphone were located in the center of the square made by the closest four 
roughness elements. A streamwise positioning diagram can be seen in Figure 2.19, with a picture of the plastic sleeves embedded in the rough surface seen in Figure 2.8.

Multiple slot locations were cut into the test surface, allowing the microphone sleeves to be moved and repositioned. This allowed the five pinhole-microphones to be positioned at 8 distinct streamwise locations and seven distinct spanwise locations along the wall, as can be seen in Figure 2.20, which is why more than 5 locations appear in Figure 2.19. Microphone spacing was arranged such that it created 45 distinct streamwise microphone separations and 37 distinct spanwise microphone separations.

The sixth unaltered reference microphone was offset in the spanwise direction as seen in Figure 2.20. This microphone was offset by a spanwise separation of $\eta / \delta=3.13$ from the streamwise microphone array and a separation between $0.74<\eta / \delta<2.81$ for microphone locations in the spanwise array when normalized on the boundary layer thickness of a smooth wall at $60-\mathrm{m} / \mathrm{s}$. The reference microphone was found to be largely uncorrelated with the other microphone positions and could thus be used for acoustic cancellation as described in section 2.6.1.

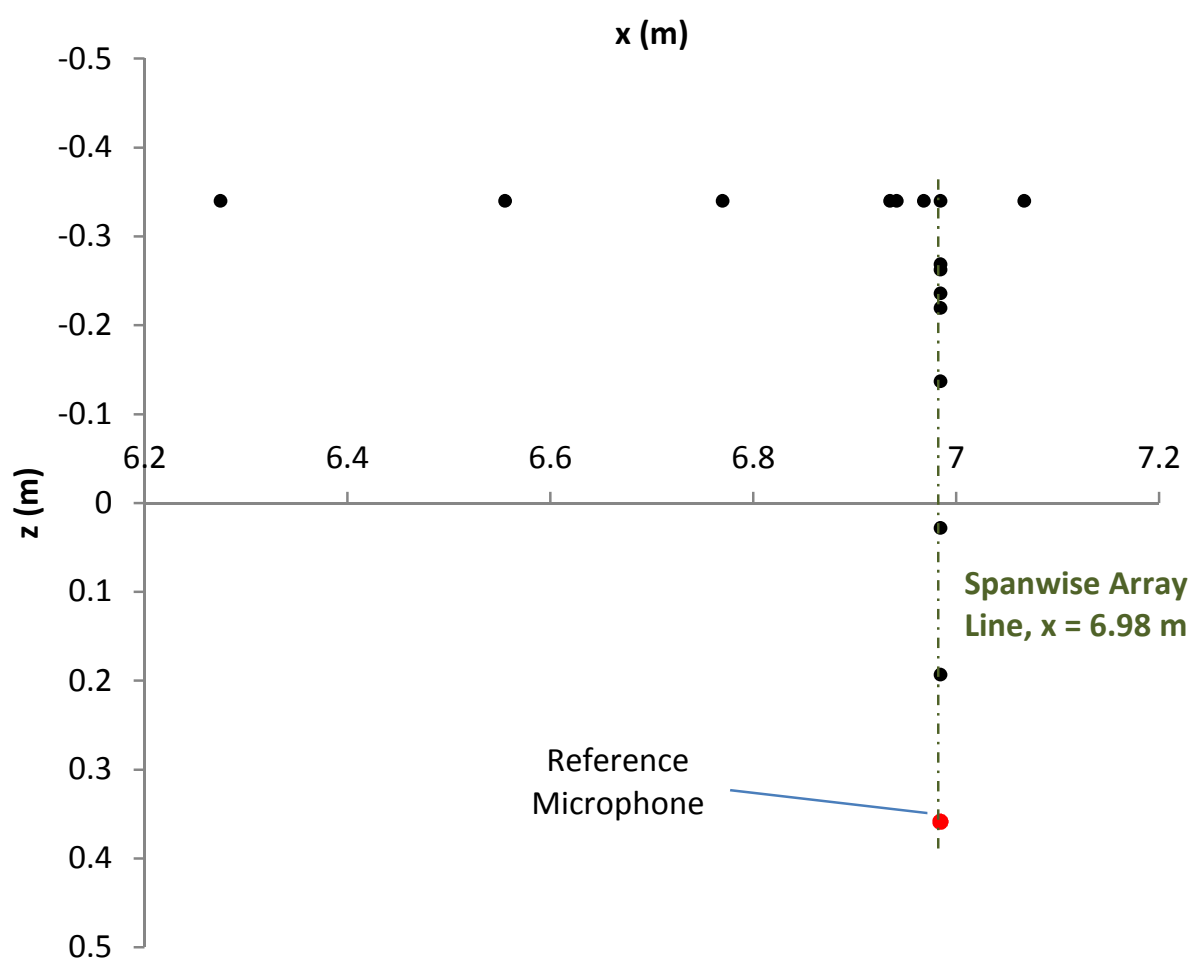

Figure 2.20. Microphone positions on the false wall for both smooth and 3-mm hemisphere roughness configurations.

\subsubsection{Microphone Calibrations}

To calibrate the amplitude and phase response of the B\&K type 4138 microphones fitted with $1 / 2-\mathrm{mm}$ pinhole caps, an anechoic chamber was configured as displayed in Figure 2.21. A University Sound ID60C8 speaker was setup along a foam covered ledge about 0.43$\mathrm{m}$ above the chamber deck and was driven by an Agilent VXI control module to produce a white noise. A narrow microphone stand was placed 2.1-m away from the speaker at 
approximately the same height and oriented using a laser pointer such that an embedded microphone would face the speaker diaphragm. An unaltered model 4138 microphone with a flat $( \pm 1-\mathrm{dB})$ frequency response up to $25.6-\mathrm{kHz}$ was then used as a reference microphone to record the speaker signal using a 16-bit Agilent E1432 data acquisition system. Subsequently, each microphone fitted with a $1 / 2-\mathrm{mm}$ pinhole cap was placed in the same location as used by the reference microphone and the speaker signal was recorded. Due to limitations in the speaker, white noise could not be produced below $250-\mathrm{Hz}$. However, the effect of the pinhole was found to be negligible for all frequencies below $1000 \mathrm{~Hz}$.

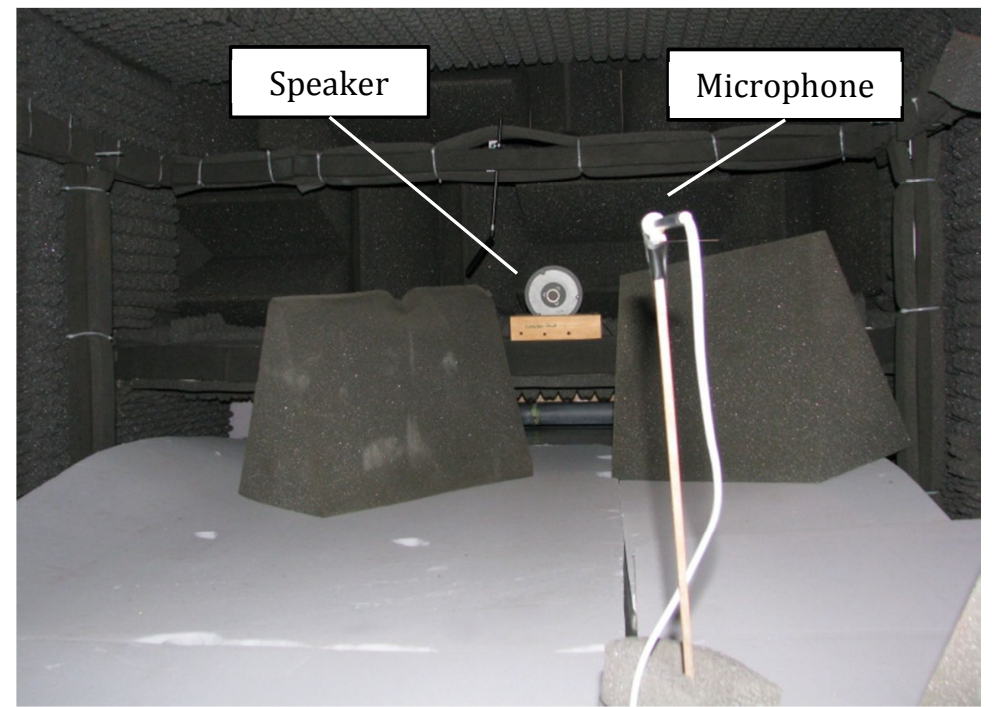

Figure 2.21. Microphone amplitude and phase calibration setup in anechoic chamber.

The initial microphone calibration was obtained using the same technique as described in Alexander (2009). Using this technique, it was found that each microphone calibration could be calculated as the response of the microphone-pinhole system divided by the response of the reference microphone. After each microphone calibration was calculated, the complex microphone frequency response was then fitted and modeled by the ratio of two polynomials, as seen in Equation 2.3.

$$
R(\omega)=\frac{b_{1} \omega^{n}+b_{2} \omega^{n-1}+\cdots+b_{n+1}}{a_{1} \omega^{2}+a_{2} \omega+a_{3}}
$$

A second order polynomial denominator was found to produce an accurate fit for all the microphones used in this study. A sample calibration and polynomial fit is displayed in Figure 2.22.

During testing, the damping effect of the pinhole on the microphone system was found to be dependent on flow speed. When there was no flow over the microphones, as was the case during calibrations, the characteristic Reynolds number of the pinhole would be very low. During testing conditions, when the system was placed under a boundary layer, the characteristic Reynolds number would increase, thus decreasing the relative magnitude of the viscous effects on the system and lowering the effective damping due to the pinhole attachment. As such, during the post process of the data, the microphone 
calibrations were optimized for flow speeds by shifting the system damping $\left(a_{2}\right)$, as well as the resonant frequency $\left(a_{1}\right)$ to account for any reverberation shift due to flow speed. It was found that the resonant frequency typically shifted by less than $4 \%$, while the damping of the pinhole-microphone system would be reduced by up to $40 \%$ at $60 \mathrm{~m} / \mathrm{s}$. Corrections at slower speeds were smaller, and negligible at $20 \mathrm{~m} / \mathrm{s}$.
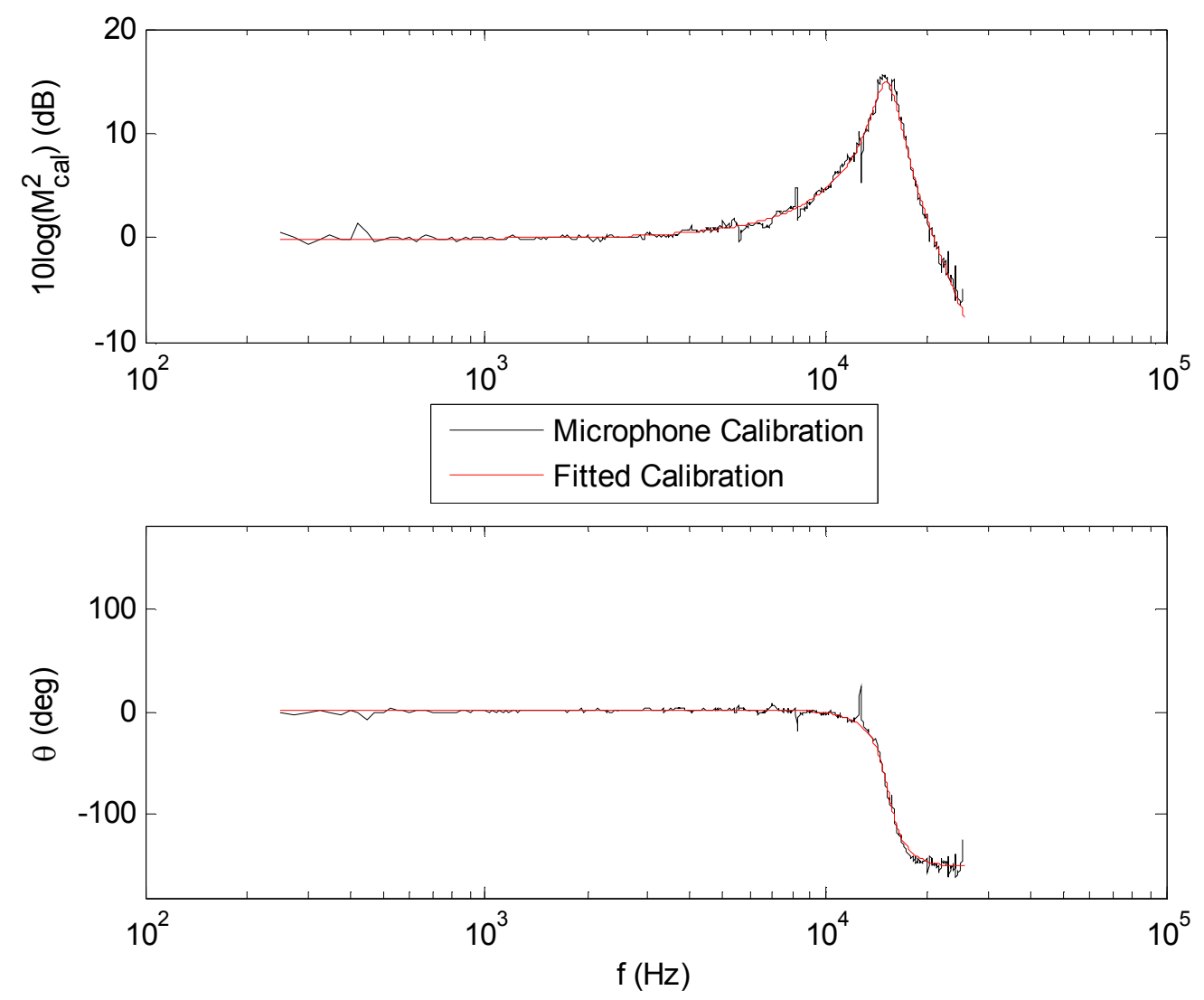

Figure 2.22. Comparison between actual and fitted microphone calibrations for amplitude and phase angle.

\subsubsection{Pulse System Filter Corrections}

The B\&K type 3050-A-060 Pulse Analyzer system used with the type 4138 microphones to collect surface pressure data was operated using a $22.4-\mathrm{Hz}$ filter during testing conditions. This filter was known to be applied by damping the system response using a frequency dependent constant for frequencies below 22.4-Hz. In order to obtain the amplitude correction factor, an Agilent VXI control module was used to produce an electronic white noise signal. The signal was split and simultaneously recorded by two different channels on a type 3050-A-060 Pulse system, with one channel using a 22.4-Hz filter and the second channel having the filter turned off. 4 sets of data sampling at 65536$\mathrm{Hz}$ over 60 seconds were recorded and averaged together. The unfiltered channel amplitude average was then divided by the filtered channel amplitude average to obtain the correction amplitude factor shown in Figure 2.23. This correction factor was found to match the given factory specifications listed for frequencies above $3-\mathrm{Hz}$. The correction factor was applied to the pressure fluctuation data by multiplying the amplitude spectra by the filter correction factor. 


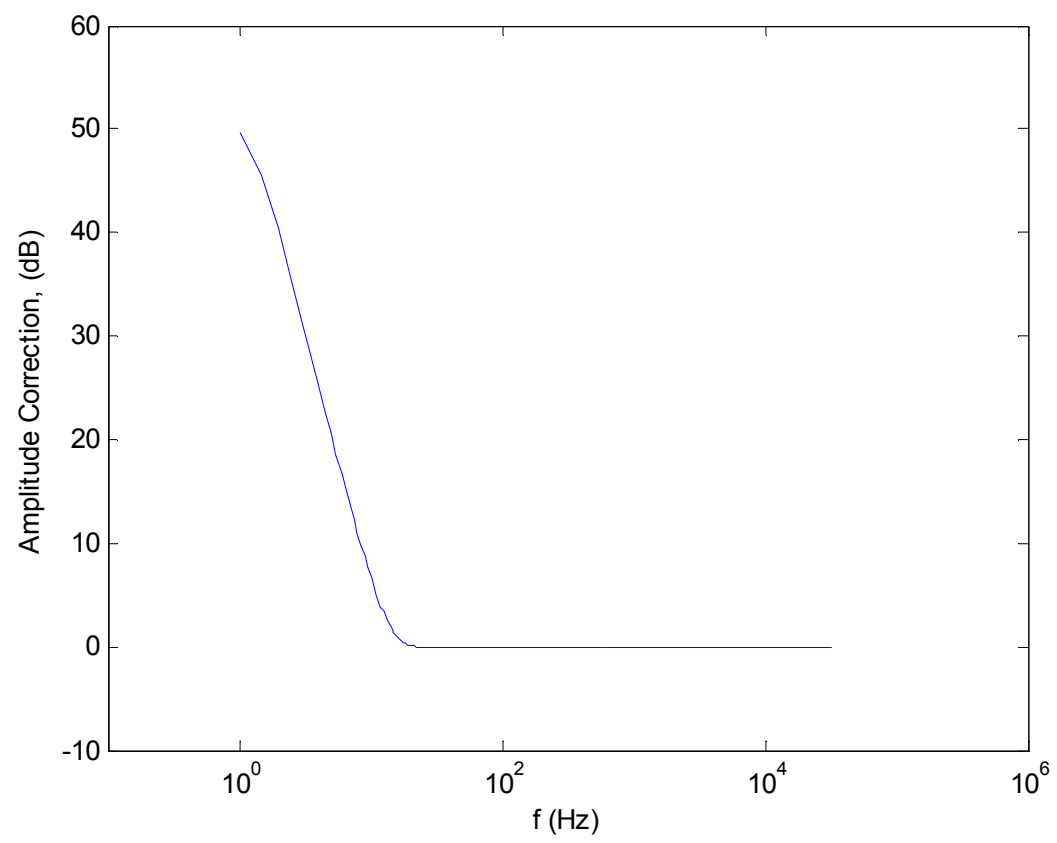

Figure 2.23. Pulse system filter correction factor.

\subsection{Discussion of Pre-Analysis Data Reduction}

\subsubsection{Acoustic Cancellation}

The spurious acoustic contributions produced by background tunnel noise were reduced through the use of noise cancellation techniques. These techniques extend the work previously done by Horne (1990) allowing for improved cross spectral acoustic subtraction.

Following Horne, one can say that the measured wall pressure spectra with acoustic noise included $\left(\phi_{p p}^{(a)}\right)$, can be expressed as the sum of the sum of the wall pressure spectra due to turbulence $\left(\phi_{p p}^{(t)}\right)$, and the wall pressure spectra due to background noise $\left(\phi_{p p}^{(n)}\right)$ as shown in Equation 2.4.

$$
\phi_{p p}^{(a)}=\phi_{p p}^{(t)}+\phi_{p p}^{(n)}
$$

Equation 2.4

When considering the wall pressure cross spectra computed between two points located at $i$ and $j$, one may write the wall pressure spectra as seen in Equation 2.5 since the noise and turbulent fluctuations will be uncorrelated.

$$
\phi_{p_{i} p_{j}}^{(a)}=\phi_{p_{i} p_{j}}^{(t)}+\phi_{p_{i} p_{j}}^{(n)}
$$

If a reference point $r$ is picked such that the turbulent pressure signal there is not correlated with the other points $i$ and $j$, then $\phi_{p_{i} p_{r}}^{(t)}=0$ would hold true. Thus to find the acoustically subtracted wall pressure autospectrum at location $i$, one may subtract the 
measured cross spectra between $i$ and $r$ from the measured autospectrum at location $i$ as seen in Equation 2.6.

$$
\phi_{p_{i} p_{i}}=\phi_{p_{i} p_{i}}^{(a)}-\phi_{p_{i} p_{r}}^{(a)}=\phi_{p_{i} p_{i}}^{(t)}+\phi_{p_{i} p_{i}}^{(n)}-\phi_{p_{i} p_{r}}^{(n)}
$$

As background noise will be the only signal correlated between locations $i$ and $r$, one can say that $\phi_{p_{i} p_{i}}^{(n)}=\phi_{p_{i} p_{r}}^{(n)}$. As such, the last two terms will cancel out and $\phi_{p_{i} p_{i}}$ will only include the wall pressure spectra due to turbulence.

This result, previously found by Horne, was expanded upon for improved acoustic subtraction when considering the cross spectral results found between two points $i$, and $j$. Mathematically, Equation 2.6 could be used when subtracting acoustics from the wall pressure cross spectra $\phi_{p_{i} p_{j}}^{(a)}$. Based on the definitions used, the reference location $r$ is uncorrelated with every other microphone position for all signals excluding the background acoustics. This implies that $\phi_{p_{i} p_{r}}^{(a)}=\phi_{p_{j} p_{r}}^{(a)}$ for all locations. However, to avoid including any possible microphone location bias, Equation 2.7 was used for acoustic subtraction. This cancellation scheme instead subtracts the average of the background acoustic signal taken between the two microphone locations in the cross spectra.

$$
\phi_{p_{i} p_{j}}=\phi_{p_{i} p_{j}}^{(a)}-\frac{1}{2}\left(\phi_{p_{i} p_{r}}^{(a)}+\phi_{p_{j} p_{r}}^{(a)}\right)
$$

Equation 2.7
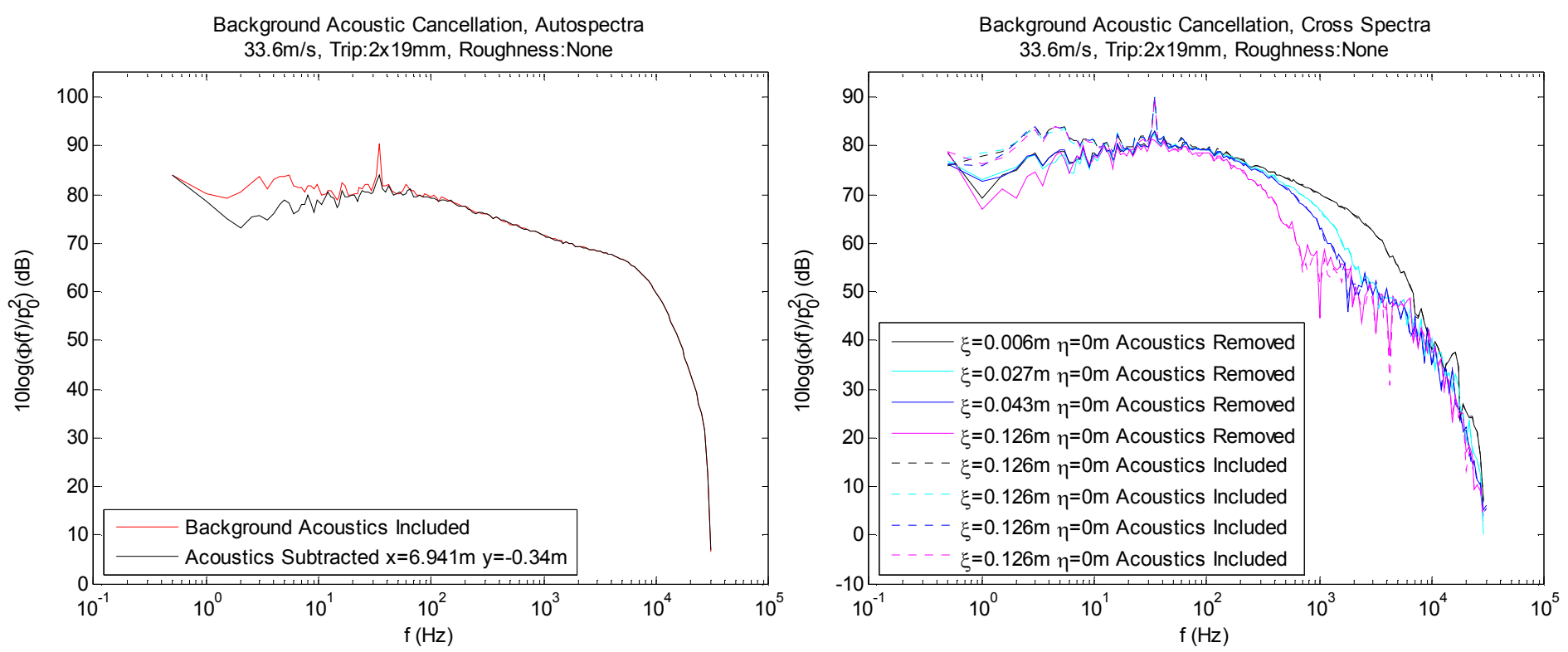

Figure 2.24. Background acoustic cancellation for select auto- and cross spectra.

This acoustic cancellation scheme was applied to all surface pressure fluctuation data as seen in Figure 2.24. Significant acoustic contamination was only found in the autospectra data below $100-\mathrm{Hz}$, with broadband contamination not exceeding $3-\mathrm{dB}$ for frequencies above $12 \mathrm{-Hz}$. The acoustic subtraction scheme was able to reduce most 
acoustic noise spikes in the pressure data, particularly a spike commonly found in the data at $34.75-\mathrm{Hz}$.

\subsubsection{Pressure Field Averaging}

During testing, surface pressure fluctuation measurements for the positions listed in Table 2.2 were repeated 4 times under each flow condition due to microphone position rotation. While combining the data from equivalent flow conditions, the collection of multiple runs at the same positions and flow states were taken advantage of with hopes of reducing uncertainties in the surface pressure fluctuation data.

Table 2.2. Repeated microphone spacing locations.

\begin{tabular}{ccccc}
\multicolumn{2}{c}{ Streamwise Array } & & \multicolumn{2}{c}{ Spanwise Array } \\
$\mathbf{x}(\mathbf{m})$ & $\mathbf{z}(\mathbf{m})$ & & $\mathbf{x}(\mathbf{m})$ & $\mathbf{z}(\mathbf{m})$ \\
\hline 6.9349 & -0.33973 & & 2.133 & -0.21903 \\
6.9414 & -0.33973 & & 2.133 & -0.23553 \\
6.9679 & -0.33973 & & 2.133 & -0.26203 \\
6.9844 & -0.33973 & & 2.133 & -0.26853
\end{tabular}

The duplicate autospectra and cross spectra data sets were combined as an arithmetic mean by frequency with sample results shown in Figure 2.25. For all data sets, it was found that the matching runs produced nearly identical pressure spectra for frequencies above $100-\mathrm{Hz}$, while at lower frequencies some increased variation was observed typically less than $3-\mathrm{dB}$ at the lowest frequencies. The uncertainties for the wall pressure spectra are estimated as \pm 2.7 - $\mathrm{dB}$ for frequencies below $25-\mathrm{Hz}, \pm 1.6-\mathrm{dB}$ for frequencies between $25-\mathrm{Hz}$ and $100-\mathrm{Hz}$, and \pm 0.9 - $\mathrm{dB}$ for frequencies above $100-\mathrm{Hz}$. The influence of the pinhole and its calibration dependence on Reynolds number also adds an increased uncertainty for the pressure spectra at high frequencies. These effects increase the uncertainty estimates to \pm 2 - $\mathrm{dB}$ for frequencies between $10-\mathrm{kHz}$ and $15-\mathrm{kHz}$, and \pm 4 - $\mathrm{dB}$ for frequencies above $15-\mathrm{kHz}$. 

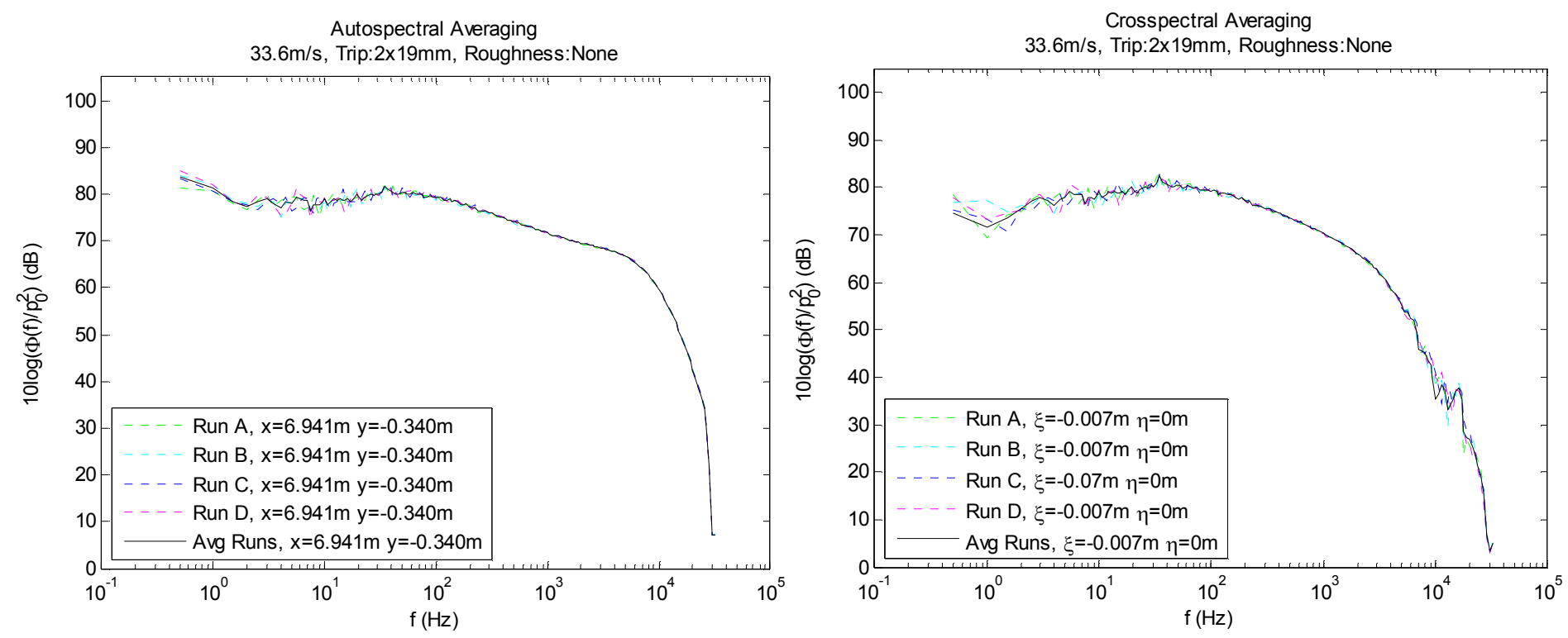

Figure 2.25. Auto- and cross spectral averaging for a select condition and microphone position.

\subsubsection{Signal Noise Filtering}

In order to ensure the quality of the presented data, the microphone pressure spectra were reduced to remove any data points that exhibited low signal to acoustic noise ratios. This was accomplished by finding the coherence between each microphone and the reference microphone. As described in section 2.6.1, the reference microphone location was offset in the spanwise direction and uncorrelated with the other microphone positions for any non-acoustic signals. Any correlation found between the reference microphone and any other microphone position was therefore considered to be due to background noise.

In order to remove frequencies which exhibited low signal to noise ratios, the coherence between each microphone location $i$ and the reference microphone location $r$ was found using the wall pressure spectra with acoustic noise included $\left(\phi_{p p}^{(a)}\right)$ as seen in Equation 2.8.

$$
\Gamma_{\text {noise }}^{2}=\frac{\left|\left(\phi_{p_{i} p_{r}}^{(a)}\right)^{2}\right|}{\left|\phi_{p_{i} p_{i}}^{(a)}\right|\left|\phi_{p_{r} p_{r}}^{(a)}\right|}
$$

For each microphone, pressure spectrum data at frequencies that were found to have $\Gamma_{\text {noise }}^{2}>0.08$ with the reference microphone were removed from the auto and cross spectra, shown in Figure 2.26. Note that in this figure, the pressure spectra has had the background acoustics removed, while the coherence and phase values are presented with respect to the background acoustic noise. 
$22.4 \mathrm{~m} / \mathrm{s}$, Trip:2x19.5mm, Roughness:None Spanwise Array
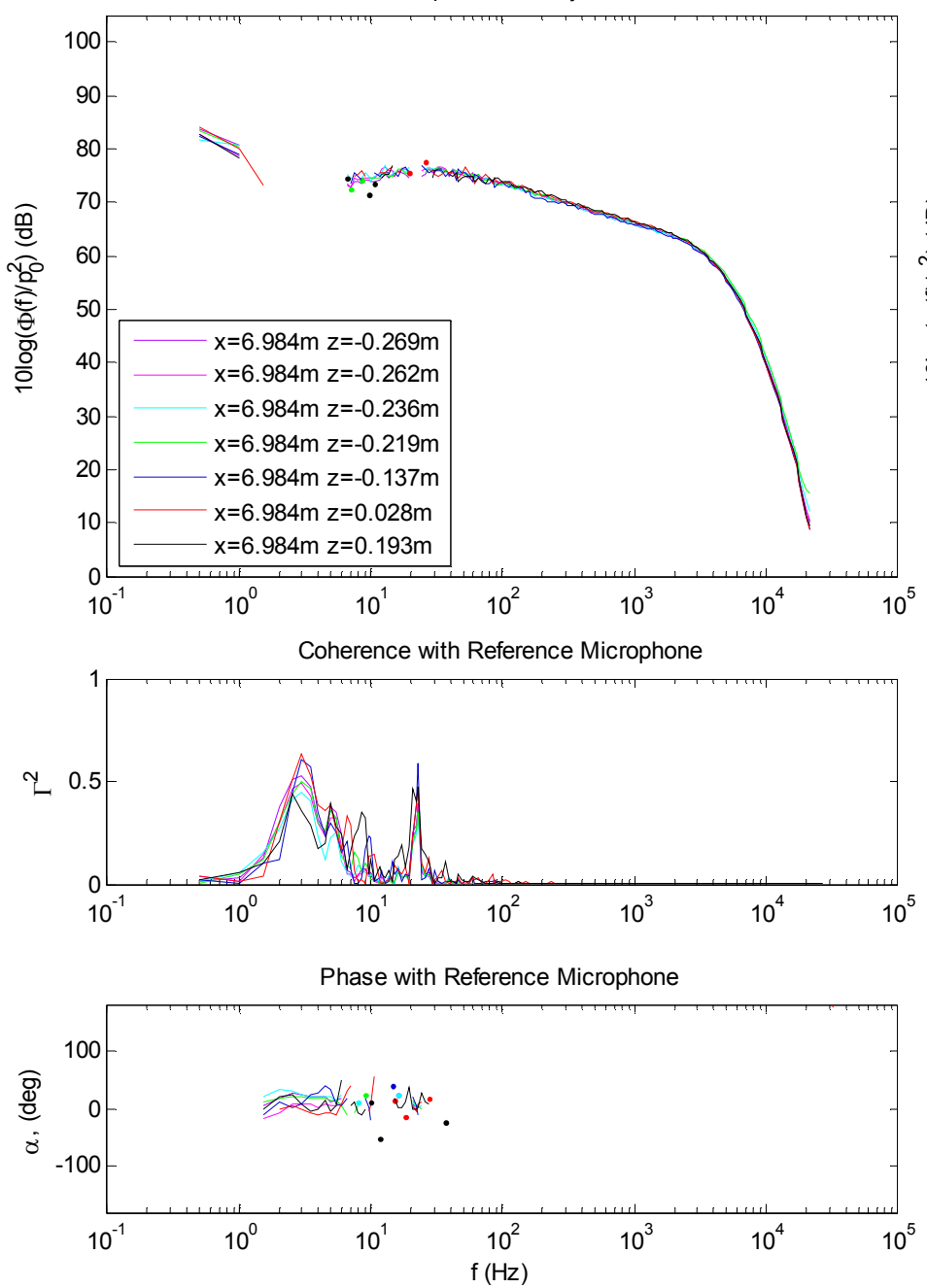

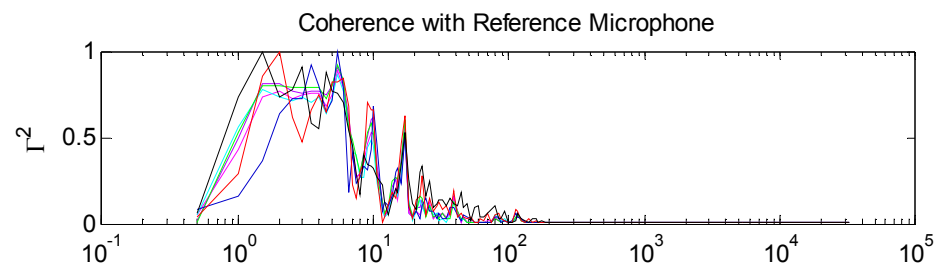

44.8m/s, Trip:2x19.5mm, Roughness:None Spanwise Array
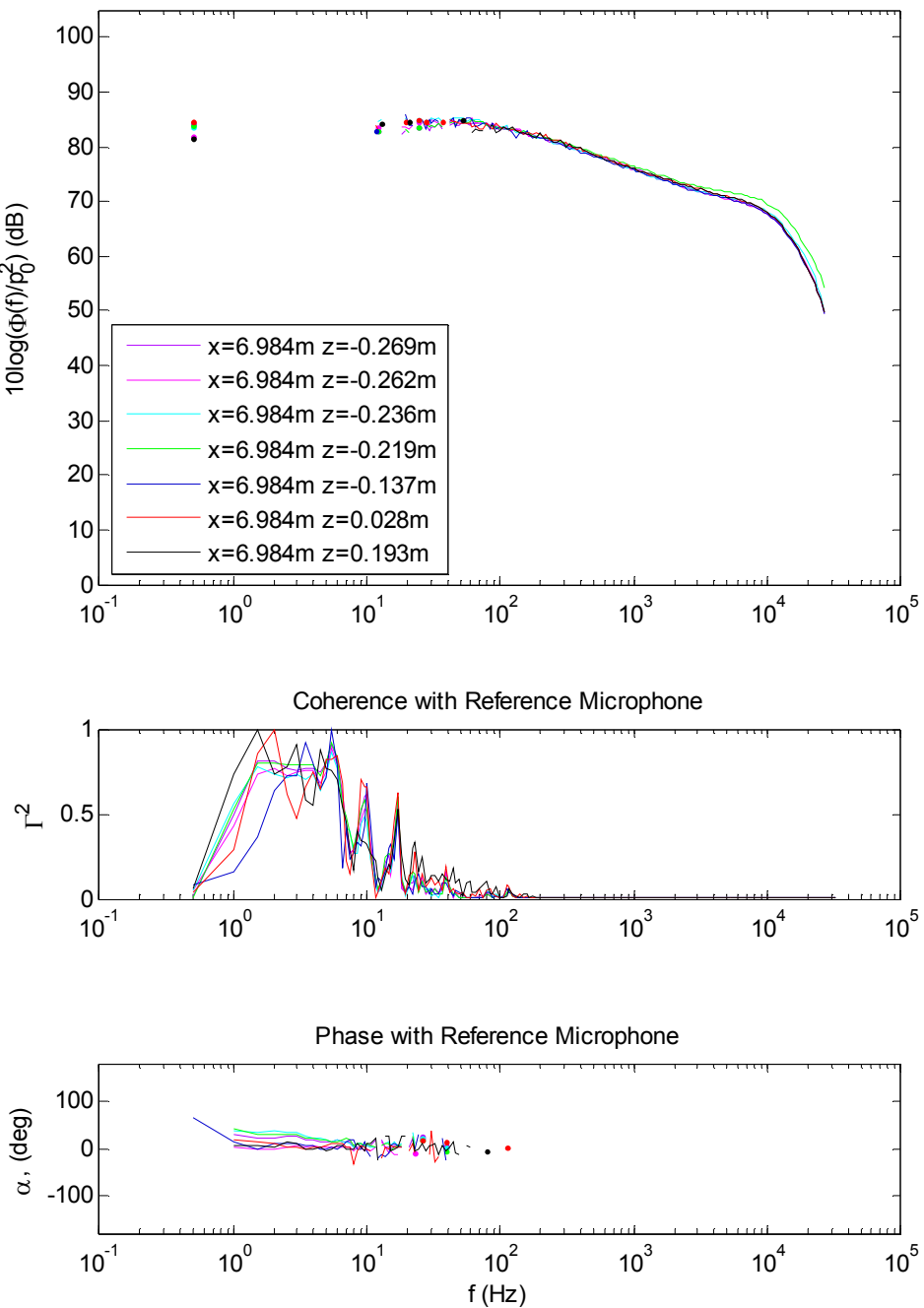

Figure 2.26. Surface pressure data with large signal noise subtracted.

The phase between the data and reference microphones is only presented for frequencies found to have a low signal to acoustic noise ratio. It was discovered that at these frequencies, the calculated phase differences were all relatively low, near $\alpha=0$. This supported the assumption that the coherence with the reference microphone was largely due to acoustics, as any turbulence data affecting the pressure fluctuations would have needed to convect downstream and thus have produced larger phase angles. 


\section{CHAPTER 3. ANALYSIS AND DISCUSSION}

\subsection{Summary of Measurements}

This primary goal of this study is to scale and characterize the wall pressure spectrum of a rough wall, high Reynolds number turbulent boundary layer free of transitional effects. Subsequently, the mean wall pressure was measured along the length of the test surface as shown in section 3.2 in order to help position the false wall and obtain a near zero-pressure gradient flow. The mean and fluctuating boundary layer velocity profiles are presented in section 3.3 in an effort to describe the flow associated with the surface pressure fluctuations. Boundary layer parameters are initially introduced and discussed in section 3.3.1, with results being used to scale the wall pressure spectra in section 3.4. It should be noted that due to testing limitations, the wall pressure fluctuation measurements could not be taken simultaneously with the velocity profiles used to obtain their associated boundary layer parameters. Because of this, some interpolative values as listed in Table 3.2 have been used with the wall pressure fluctuation measurements. The features, characteristics and scaling parameters found for the single-point wall pressure spectra are presented in sections 3.4.1 through 3.4.3, while the progression of different features of the two-point wall pressure spectra are characterized in sections 3.4.4 through 3.4.6.

\subsection{Mean Wall Pressure Gradient}

The mean wall pressure was measured at 24 locations along the floor and ceiling of the anechoic test section to obtain the pressure gradient along the false wall. Initially, these measurements were used to adjust the false wall position to minimize the wall pressure gradient. The pressure gradient for the final wall position is shown in Figure 3.1. Smooth and rough wall mean pressures in flows ranging from $30 \mathrm{~m} / \mathrm{s}$ to $60 \mathrm{~m} / \mathrm{s}$ generally were kept constant to within $\pm 0.03 C_{p}$. The slight favorable pressure gradient over the downstream half of the wall was believed to be due to boundary layer growth on the Kevlar wall on the opposite side of the test section. Physical restrictions prevented the wall adjustment needed to completely cancel this. 


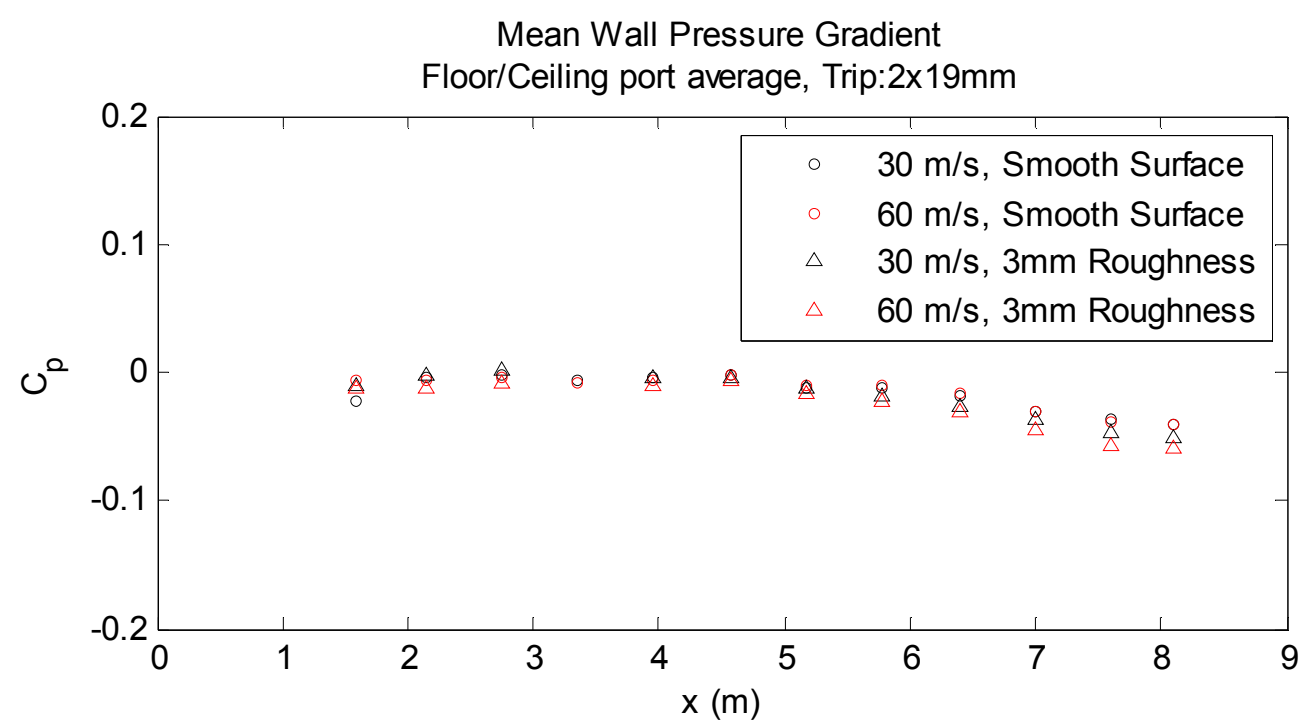

Figure 3.1. Mean surface pressure across hard wall testing surface.

The wall position was not re-adjusted with change in flow speed, or with the addition of roughness. It can be seen in Figure 3.1 that flow velocity had a negligible effect on the mean wall pressure. The addition of the 3-mm hemispherical roughness did have a slight effect on the mean wall pressures at larger distances downstream, though first indications of this do not appear until 5.8-m downstream of the trip. Here the $C_{p}$ of the rough wall differs from that of the smooth by less than 0.008 for all flow speeds. While the mean pressure differential between smooth and rough walls does continue to increase downstream of this location, the difference in pressure coefficients between smooth and rough walls never exceeded 0.019 . For the presented results, the pressure gradients were both treated as negligible with the mean wall pressures for smooth and rough walls being treated as equal.

\subsection{Wall Flow Boundary Layer}

\subsubsection{General Boundary Layer Form}

Boundary layer profiles were measured along the wall using a flat-head Pitot and a 4-component hotwire anemometer in order to determine the boundary layer characteristics. All boundary layers considered in this study were fully developed and turbulent. Near-wall behavior of the smooth and rough wall boundary layers was assumed to be a function of position $y$, friction velocity $u_{t}$, and kinematic viscosity $v$ as defined by the law of the wall in Equation 3.1 (Schetz 1993).

$$
\frac{u}{u_{t}}=f\left(\frac{y u_{t}}{v}\right)
$$

The overlap or logarithmic region of the flow for smooth wall as assumed to follow Equation 3.2 where, according to Coles (1956), the Von Kármán constant $\kappa=0.40$ and $B=$ 5.1. 


$$
\frac{u}{u_{t}}=\frac{1}{\kappa} \ln \left(\frac{y u_{t}}{v}\right)+B
$$

For flow over rough walls, the laminar sublayer disappears and the equivalent sand grain roughness height $k_{s}$ becomes an important scaling factor. The equivalent sand grain roughness can be found using Equation 3.3 where $\Delta u / u_{t}$ is the roughness defect shift away from the smooth wall data due to roughness effects. The constant $B=5.1$ was used as before, with the constant $C$ being dependent on the roughness.

$$
\frac{\Delta u}{u_{t}}=-\frac{1}{\kappa} \ln \left(\frac{k_{s} u_{t}}{v}\right)-B+C
$$

Following Schlichting (1979), the roughness function $C$ can be represented as a function of the equivalent sand grain roughness height Reynolds number $k_{s}{ }^{+}$. While implicit methods may be used to solve Equation 3.3 for the equivalent sand grain roughness, it is also known that the roughness function $C$ becomes a constant for fully rough flows which are predicted for $k_{s}{ }^{+}>70$. In order to obtain an initial estimate for $k_{s}$ and thus $k_{s}{ }^{+}$, Equation 3.4 was used to correlate the equivalent sand grain roughness height $k_{s}$ to the mean roughness height $k_{g}$ (Dirling 1973). In this equation, $d_{e}$ is the mean distance between roughness elements, $A_{p}$ is the projected roughness surface area onto a plane normal to the flow direction, and $A_{s}$ is the wetted surface area of the roughness as seen by the flow.

$$
\begin{aligned}
k_{s} \approx \frac{k_{g}}{\vartheta}, \quad \vartheta & = \begin{cases}60.95 \Lambda^{-3.78} & \text { if } \Lambda<4.915 \\
0.0072 \Lambda^{1.9} & \text { if } \Lambda>4.915\end{cases} \\
\Lambda & =\frac{d_{e}}{k_{g}}\left(\frac{A_{s}}{A_{p}}\right)^{\frac{4}{3}}
\end{aligned}
$$

Using Equation 3.4, one finds that for the roughness used in this study, the theorized equivalent sand grain roughness height $k_{s}=2.82-\mathrm{mm}$. At 30 and $60-\mathrm{m} / \mathrm{s}$ using two 19-mm trips, this equated to theorized equivalent sand grain roughness height Reynolds numbers of $k_{s}{ }^{+}=266$ and $k_{s}{ }^{+}=477$ respectively, both well within the fully rough regime $\left(k_{s}{ }^{+}>70\right)$. Following Schlichting (1979), a roughness function of $C=8.5$ could thus be used to solve for the actual value of $k_{s}$ in Equation 3.3 due to the fully rough regime the flow resided in. Final results for the equivalent sand grain roughness heights can be seen in Table 3.3.

Once the equivalent sand grain roughness height is known, one can define the overlap region of the inner boundary layer flow for rough walls as seen in Equation 3.5 (Schlichting 1979). This is the equivalent law of the wall for rough surfaces when compared to Equation 3.2 for smooth surface flow.

$$
\begin{aligned}
& \frac{u}{u_{t}}=\frac{1}{\kappa} \ln \left(\frac{y u_{t}}{v}\right)+D \\
& D=-\frac{1}{\kappa} \ln \left(\frac{k_{s} u_{t}}{v}\right)+C
\end{aligned}
$$

Equation 3.5 
Note that the rough surface law of the wall from Equation 3.5 still conforms to Equation 3.1 as a function of $y, u_{t}$, and $v$. For the fully rough flows studied, the roughness function constant $C=8.5$ as discussed earlier and the Von Kármán constant $\kappa=0.40$ from Coles (1956) was used. Additionally, Equation 3.5 can be further reduced to Equation 3.6, where the velocity is not as clearly dependent on $u_{t}$ and $v$, though these variables are accounted for when solving for $k_{s}$ from Equation 3.3.

$$
\frac{u}{u_{t}}=\frac{1}{\kappa} \ln \left(\frac{y}{k_{s}}\right)+C
$$

Equation 3.6

One can define law of the wall position and velocity variables $y^{+}$and $u^{+}$respectively as seen in Equation 3.7. By normalizing the collected smooth and rough wall data by these definitions, one can determine the flow friction velocity $u_{t}$ by fitting the theorized overlap regions of Equation 3.2 and Equation 3.6 with the smooth and rough wall experimental data normalized by Equation 3.7.

$$
\begin{gathered}
y^{+} \equiv \frac{y u_{t}}{v} \quad u^{+} \equiv \frac{u}{u_{t}} \\
u_{t}=\sqrt{\frac{\tau_{w}}{\rho}}=\sqrt{\frac{1}{2} C_{f} U_{e}^{2}}
\end{gathered}
$$

The law of the wall results are shown in Figure 3.2, and verified through analyzing the Reynolds stress profiles as discussed section 3.3.2. Skin friction coefficient values $C_{f}$ can be related to friction velocity $u_{t}$ by Equation 3.8. These values are shown in Table 3.1. 


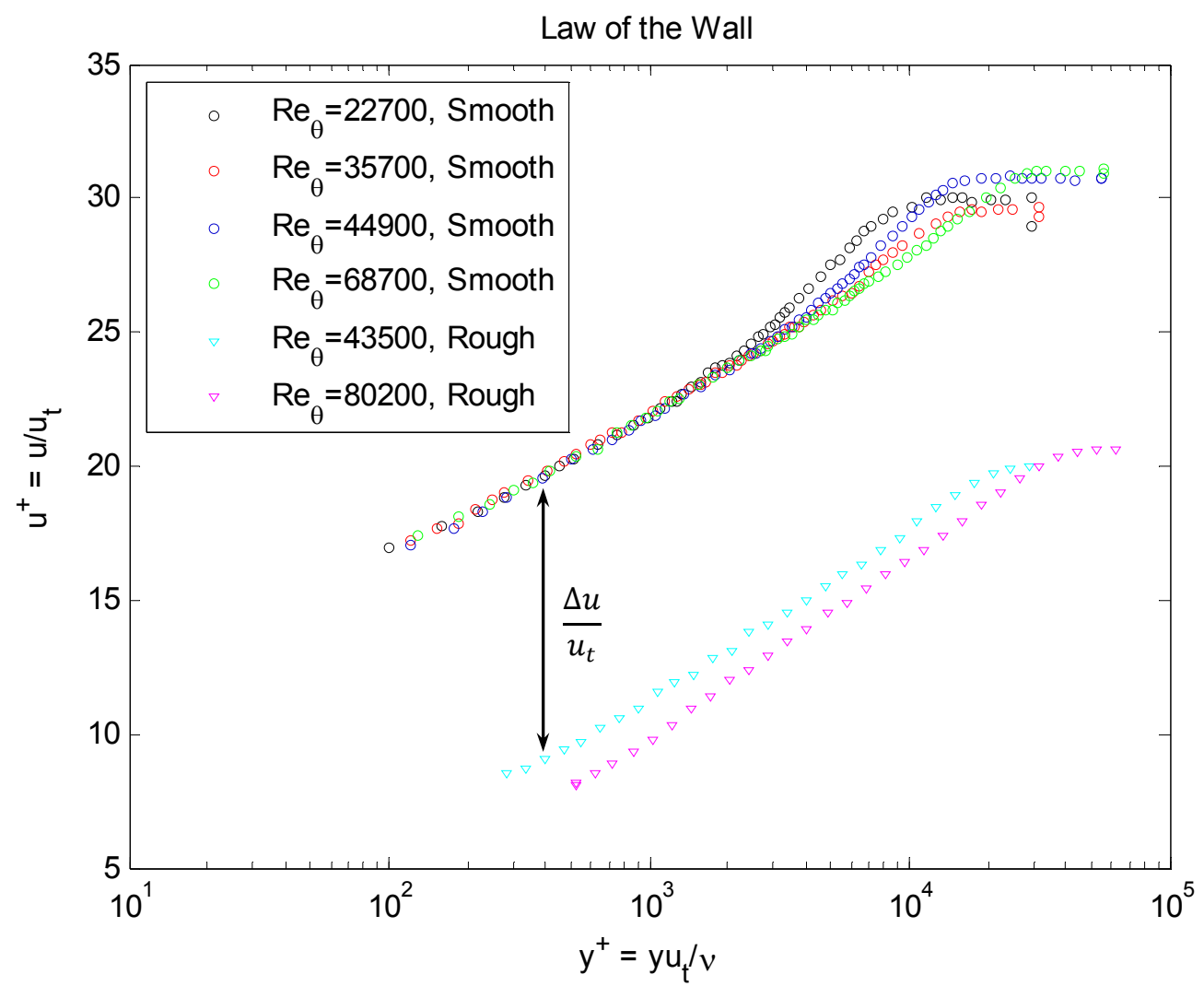

Figure 3.2. Law of the wall for smooth and rough walls.

Table 3.1 lists the major boundary layer parameters for both smooth and rough wall flows at various conditions. The primary flow conditions at which turbulent flow velocity and pressure fluctuations data are based are at 30 and $60-\mathrm{m} / \mathrm{s}, 7.0-\mathrm{m}$ downstream of the two 19-mm trips. These data entries have been bolded in the table. Note that the rough wall data in Table 3.1 was taken at a later date with ambient temperatures averaging $19.4^{\circ}-\mathrm{C}$ lower than the smooth wall conditions. This resulted in lower flow kinematic viscosities and thus comparatively larger Reynolds numbers. This explains why the listed boundary layer thickness $\delta$ is smaller for the same conditions over a rough surface when compared to the smooth wall boundary layer thickness. However, despite the decreased boundary layer thickness for the rough wall flows, $R_{\delta}$ is very comparable between equivalent conditions for both smooth and rough walls. 
Table 3.1. Boundary layer parameters for flow over a zero-pressure gradient wall.

\begin{tabular}{cccccccccccc}
$\begin{array}{c}\text { Speed } \\
(\mathbf{m} / \mathbf{s})\end{array}$ & $\begin{array}{c}\text { Trip } \\
(\mathbf{m m})\end{array}$ & $\mathbf{X}(\mathbf{m})$ & $\boldsymbol{\delta}(\mathbf{m})$ & $\mathbf{C}_{\mathbf{f}}$ & $\mathbf{u}_{\mathbf{t}} / \mathbf{U}_{\mathbf{e}}$ & $\boldsymbol{\delta}^{*}(\mathbf{m})$ & $\boldsymbol{\Theta}(\mathbf{m})$ & $\mathbf{R e}_{\boldsymbol{\delta}}$ & $\mathbf{R e}_{\boldsymbol{\Theta}}$ & $\mathbf{H}$ & Configuration \\
\hline 30 & 9.5 & 4.7 & 0.123 & 0.00254 & 0.0356 & 0.0141 & 0.0111 & 214000 & 19400 & 1.27 & Smooth \\
30 & $\mathbf{2 \times 1 9}$ & 4.7 & 0.210 & 0.00242 & 0.0348 & 0.0220 & 0.0179 & 371000 & 31600 & 1.23 & Smooth \\
30 & 9.5 & 5.0 & 0.133 & 0.00247 & 0.0351 & 0.0146 & 0.0115 & 232000 & 20200 & 1.27 & Smooth \\
30 & 9.5 & 5.4 & 0.131 & 0.00243 & 0.0349 & 0.0151 & 0.0118 & 228000 & 20600 & 1.27 & Smooth \\
30 & 9.5 & 7.0 & 0.138 & 0.00235 & 0.0343 & 0.0176 & 0.0134 & 233000 & 22700 & 1.32 & Smooth \\
30 & 9.5 & 7.0 & 0.165 & 0.00505 & 0.0502 & 0.0329 & 0.0214 & 272000 & 35200 & 1.54 & 3-mm Rough \\
$\mathbf{3 0}$ & $\mathbf{2 \times 1 9}$ & $\mathbf{7 . 0}$ & $\mathbf{0 . 2 3 1}$ & $\mathbf{0 . 0 0 2 3 2}$ & $\mathbf{0 . 0 3 4 1}$ & $\mathbf{0 . 0 2 4 6}$ & $\mathbf{0 . 0 1 9 8}$ & $\mathbf{4 1 9 0 0 0}$ & $\mathbf{3 5 7 0 0}$ & $\mathbf{1 . 2 5}$ & Smooth \\
$\mathbf{3 0}$ & $\mathbf{2 \times 1 9}$ & $\mathbf{7 . 0}$ & $\mathbf{0 . 2 1 9}$ & $\mathbf{0 . 0 0 5 0 2}$ & $\mathbf{0 . 0 5 0 1}$ & $\mathbf{0 . 0 3 2 1}$ & $\mathbf{0 . 0 2 3 1}$ & $\mathbf{4 1 2 0 0 0}$ & $\mathbf{4 3 5 0 0}$ & $\mathbf{1 . 3 9}$ & 3-mm Rough \\
60 & 9.5 & 4.7 & 0.115 & 0.00225 & 0.0335 & 0.0112 & 0.0094 & 397000 & 32500 & 1.19 & Smooth \\
60 & $\mathbf{2 \times 1 9}$ & 4.7 & 0.220 & 0.00217 & 0.0329 & 0.0212 & 0.0177 & $\mathbf{7 5 8 0 0 0}$ & 61000 & 1.20 & Smooth \\
60 & 9.5 & 5.0 & 0.128 & 0.00225 & 0.0335 & 0.0143 & 0.0115 & 440000 & 39600 & 1.25 & Smooth \\
60 & 9.5 & 5.4 & 0.130 & 0.00222 & 0.0333 & 0.0149 & 0.0119 & 452000 & 41400 & 1.25 & Smooth \\
60 & 9.5 & 7.0 & 0.137 & 0.00212 & 0.0326 & 0.0179 & 0.0137 & 451000 & 44900 & 1.31 & Smooth \\
$\mathbf{6 0}$ & $\mathbf{2 \times 1 9}$ & $\mathbf{7 . 0}$ & $\mathbf{0 . 2 3 1}$ & $\mathbf{0 . 0 0 2 0 8}$ & $\mathbf{0 . 0 3 2 2}$ & $\mathbf{0 . 0 2 4 9}$ & $\mathbf{0 . 0 2 0 0}$ & $\mathbf{7 9 3 0 0 0}$ & $\mathbf{6 8 7 0 0}$ & $\mathbf{1 . 2 4}$ & Smooth \\
$\mathbf{6 0}$ & $\mathbf{2 \times 1 9}$ & $\mathbf{7 . 0}$ & $\mathbf{0 . 2 2 8}$ & $\mathbf{0 . 0 0 4 6 8}$ & $\mathbf{0 . 0 4 8 4}$ & $\mathbf{0 . 0 3 1 9}$ & $\mathbf{0 . 0 2 3 0}$ & $\mathbf{7 9 6 0 0 0}$ & $\mathbf{8 0 2 0 0}$ & $\mathbf{1 . 3 9}$ & 3-mm Rough \\
60 & 9.5 & 7.6 & 0.142 & 0.00212 & 0.0326 & 0.0160 & 0.0129 & 509000 & 46400 & 1.24 & Smooth \\
60 & $\mathbf{2 \times 1 9}$ & 7.6 & 0.246 & 0.00204 & 0.0319 & 0.0248 & 0.0204 & 881000 & $\mathbf{7 3 0 0 0}$ & 1.22 & Smooth
\end{tabular}

Testing limitations prevented many of the wall pressure fluctuation measurements from being taken under the same flow conditions as those presented in Table 3.1. As the needed flow conditions were largely bracketed by the measurements shown in this table, the boundary layer parameters used in section 3.4 for were interpolated as seen in Table 3.2. Note that bolded entries in Table 3.2 have not been interpolated, but come directly from Table 3.1.

Table 3.2. Interpolated boundary layer parameters used for wall pressure scaling in section 3.4.

\begin{tabular}{|c|c|c|c|c|c|c|c|c|c|c|c|}
\hline $\begin{array}{c}\text { Speed } \\
(\mathrm{m} / \mathrm{s})\end{array}$ & $\begin{array}{l}\text { Trip } \\
(\mathrm{mm})\end{array}$ & $X(m)$ & $\delta(\mathrm{m})$ & $C_{f}$ & $u_{t} / U_{e}$ & $\delta^{*}(m)$ & $\Theta(m)$ & $\mathbf{R e}_{\delta}$ & $\mathbf{R e}_{\odot}$ & H & Configuration \\
\hline 22.4 & $2 \times 19$ & 7.0 & 0.232 & 0.00238 & 0.03450 & 0.0245 & 0.0197 & 324000 & 27400 & 1.25 & Smooth \\
\hline 33.6 & $2 \times 19$ & 7.0 & 0.231 & 0.00229 & 0.03385 & 0.0246 & 0.0198 & 464000 & 39700 & 1.25 & Smooth \\
\hline 44.8 & $2 \times 19$ & 7.0 & 0.231 & 0.00220 & 0.03318 & 0.0247 & 0.0199 & 603000 & 52000 & 1.25 & Smooth \\
\hline 56 & $2 \times 19$ & 7.0 & 0.231 & 0.00211 & 0.03250 & 0.0248 & 0.0200 & 743000 & 64300 & 1.24 & Smooth \\
\hline 67.2 & $2 \times 19$ & 7.0 & 0.230 & 0.00202 & 0.03180 & 0.0249 & 0.0201 & 883000 & 76700 & 1.24 & Smooth \\
\hline 20 & $2 \times 19$ & 7.0 & 0.215 & 0.00513 & 0.05065 & 0.0322 & 0.0231 & 284000 & 31200 & 1.39 & 3-mm Rough \\
\hline 30 & $2 \times 19$ & 7.0 & 0.219 & 0.00502 & 0.05009 & 0.0321 & 0.0231 & 412000 & 43500 & 1.39 & 3-mm Rough \\
\hline 40 & $2 \times 19$ & 7.0 & 0.222 & 0.00491 & 0.04952 & 0.0320 & 0.0230 & 540000 & 55700 & 1.39 & 3-mm Rough \\
\hline 50 & $2 \times 19$ & 7.0 & 0.225 & 0.00479 & 0.04895 & 0.0319 & 0.0230 & 668000 & 68000 & 1.39 & 3-mm Rough \\
\hline 60 & $2 \times 19$ & 7.0 & 0.228 & 0.00468 & 0.04837 & 0.0319 & 0.0230 & 796000 & 80200 & 1.39 & 3-mm Rough \\
\hline
\end{tabular}

The smooth wall skin friction coefficient values shown in Table 3.1 are compared to previous studies (Blake 1970, Fernholz and Finley 1996, Österlund et al. 2003) over a large range of momentum thickness based Reynolds numbers for zero-pressure gradient smooth surfaces in Figure 3.3. Here one can see the close agreement found with other smooth wall 
data at similar $R e_{\theta}$. Skin friction value for rough wall flows are much more difficult to compare due to the strong dependence on the type of roughness used.

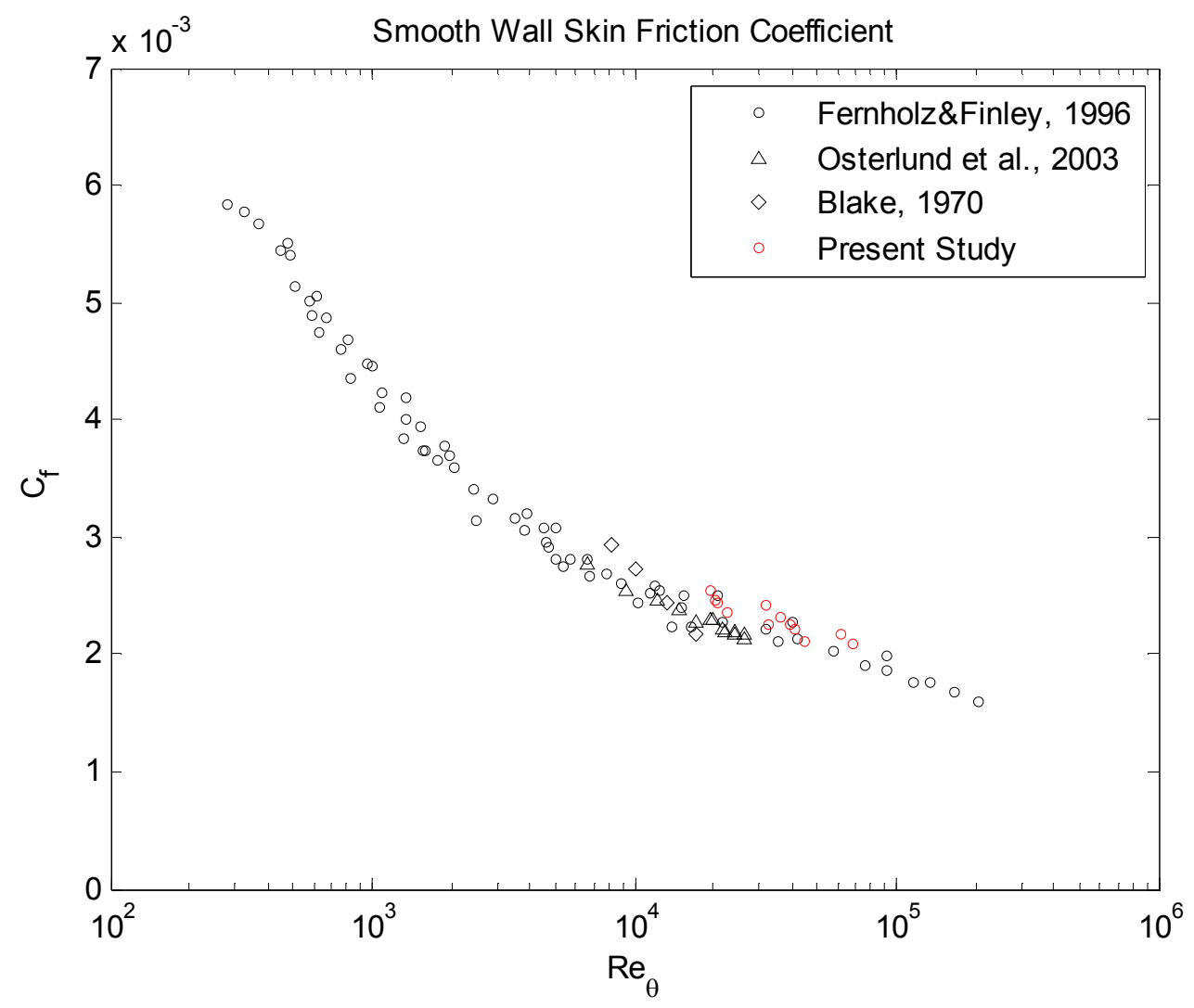

Figure 3.3. Skin friction coefficient comparison to other zero-pressure gradient smooth wall data.
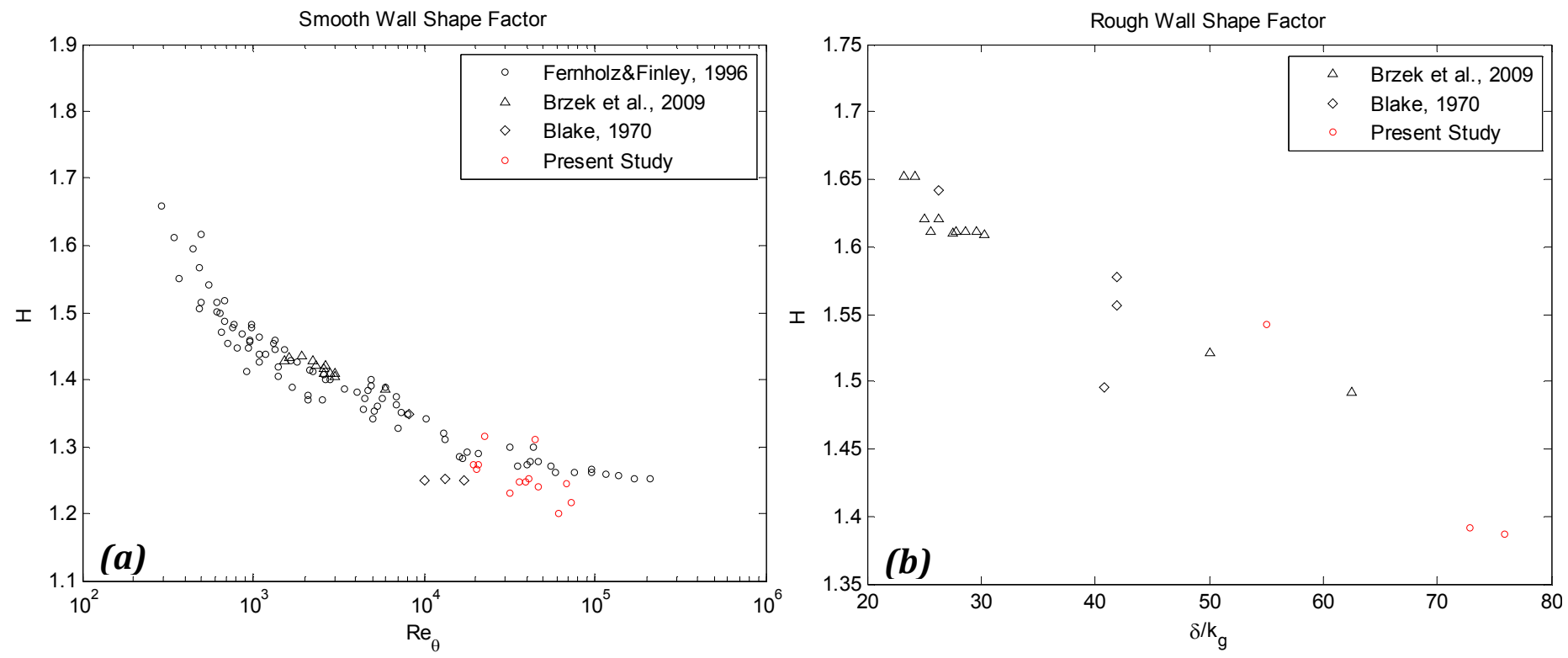

Figure 3.4. Boundary layer shape factor comparisons for (a) smooth and (b) rough wall data. 
The turbulent boundary layer shape factor $H$ in Table 3.1 is compared to other smooth and rough wall zero-pressure gradient data in Figure 3.4. On the left, the smooth wall shape factor data is related to the momentum thickness based Reynolds number. The present study's findings were found to be in general agreement with the smooth wall data from Blake (1970), Fernholz and Finley (1995), and Brzek et al. (2009).

The shape factor comparisons for the rough wall turbulent boundary layer can be seen at the right of Figure 3.4. Boundary layer shape factor is largely dependent on the local skin friction coefficient $C_{f}$, which is why for smooth wall turbulent boundary layers the shape factor at least appears to correlate well with $\operatorname{Re}_{\theta}$. For fully rough flows, the relative roughness height usually contributes more to the skin friction than the flow Reynolds number. Therefore, to make a more accurate comparison, the boundary layer shape factor has been plotted in relationship to the boundary layer thickness to roughness height ratio $\delta / k_{g}$ for rough walls at the right of Figure 3.4. When presented in this way, the present study's findings align with the general trend of rough wall data as found by Blake (1970) and Brzek et al. (2009).

The effects of roughness on the boundary layer shape can clearly be seen in Figure 3.5. The change in shape of the boundary layer as suggested by the roughness's effect on shape factor $H$ is clearly evident, as the increased wall shear due to roughness slows the boundary layer mean flow velocity out until near $y / \delta=0.6$.
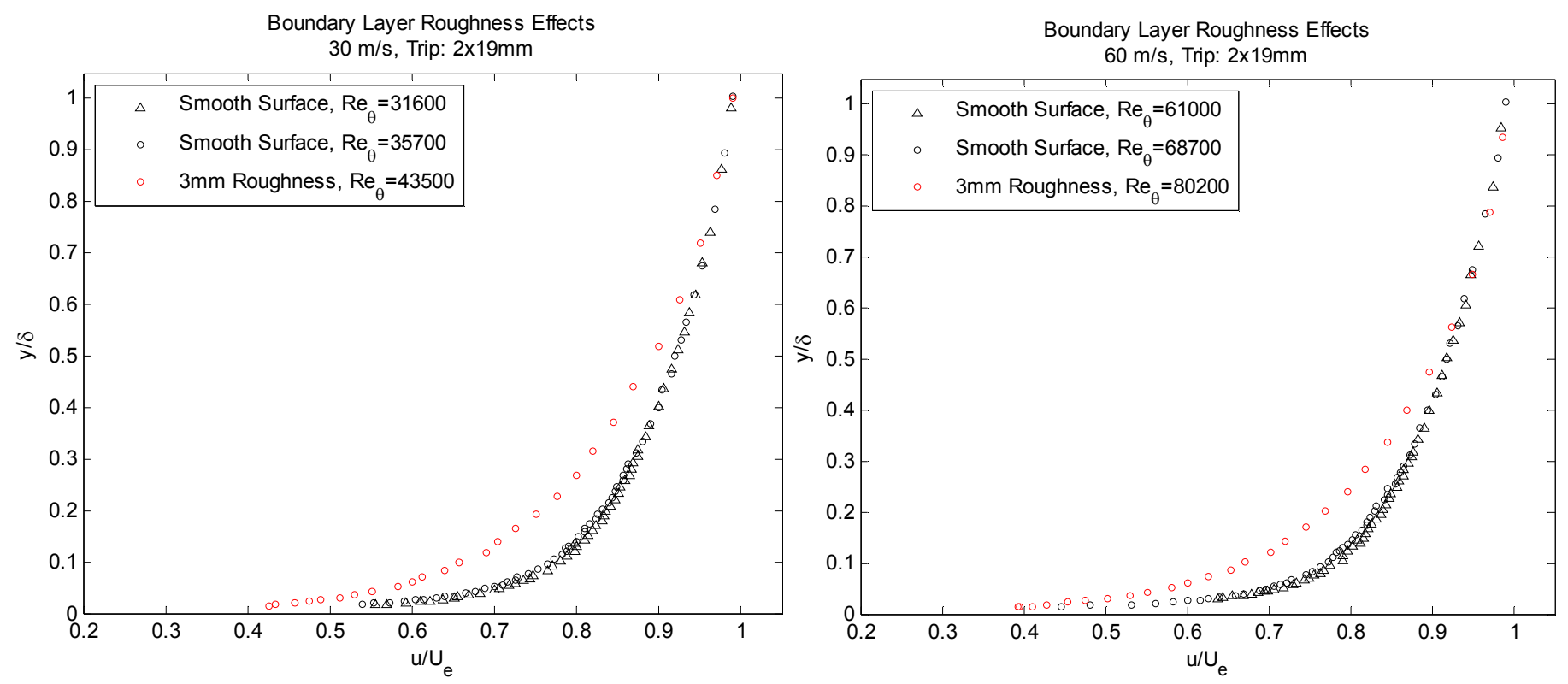

Figure 3.5. 3-mm roughness effects on boundary layer profile shape.

To check the two-dimensionality of the boundary layer flow development, boundary layer profiles were taken on the smooth surface along the centerline of the tunnel and at spanwise locations of $z= \pm 0.46$-m. Figure 3.6 shows these profiles for the double 19 -mm trip configurations at 30 and $60 \mathrm{~m} / \mathrm{s}$ at locations $4.7-\mathrm{m}$ and $7.0-\mathrm{m}$ downstream of the primary. The boundary layer profiles at the different spanwise locations were found to be similar enough for the flow to be treated as two-dimensional. 

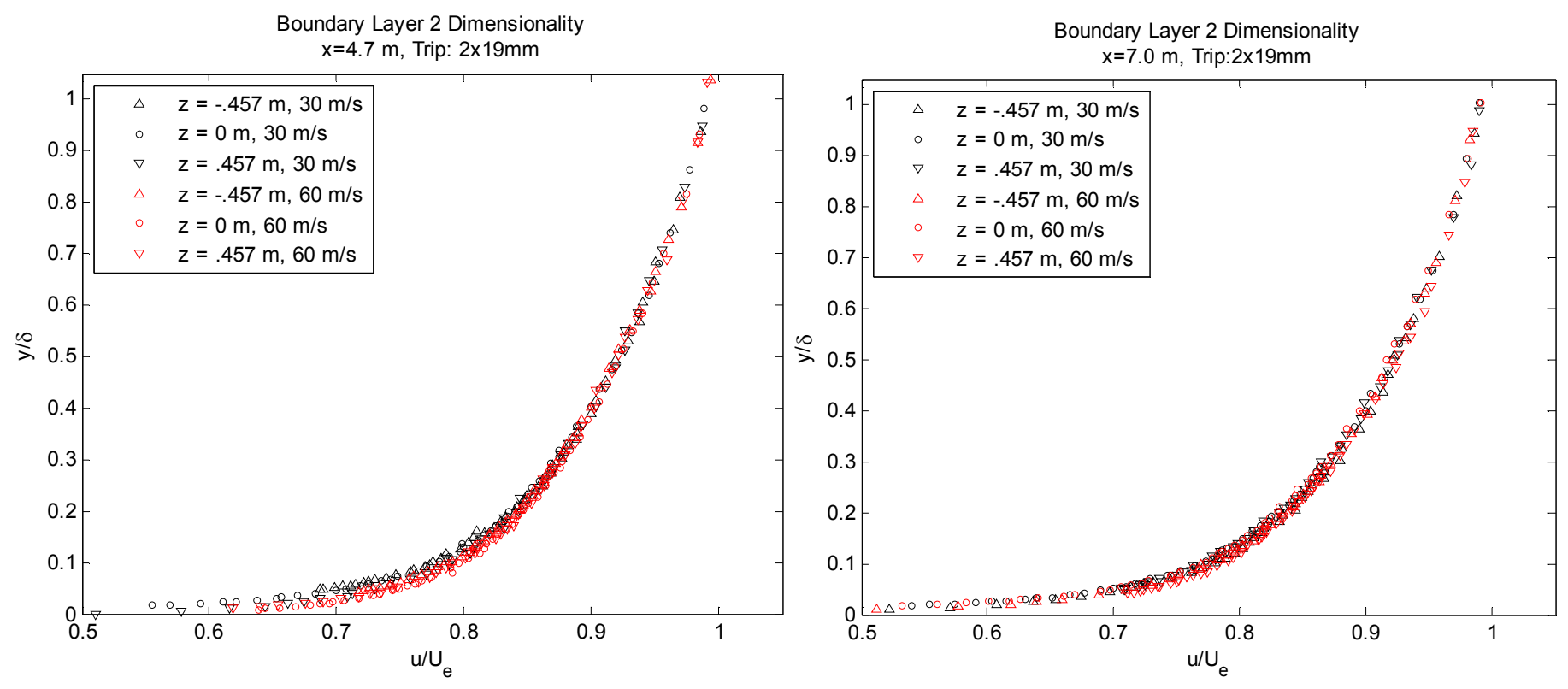

Figure 3.6. Boundary layer two-dimensionality.

Table 3.3 displays various roughness parameters for flows over a zero-pressure gradient rough wall for both the present and comparable studies. Note that the minor variation in the $k_{s}$ values from the present study's single roughness configuration can be attributable to experimental error. Of all the flows found to be fully rough $\left(k_{s}{ }^{+}>70\right.$, and Varano's 2010 study) using Schlichting's (1979) requirements, only the configurations in the present study and a single case as studied by Aupperle and Lambert (1970) meet the criteria set by Jiménez (2004) to be free of transitional effects. These stipulations require a roughness height Reynolds number $k_{g}{ }^{+}>80$, and a blockage ratio of $\delta / k_{g}>50$. 
Table 3.3. Roughness parameters for flow over a zero-pressure gradient rough wall. Data from previous studies is also listed for comparison.

\begin{tabular}{|c|c|c|c|c|c|c|c|c|c|}
\hline & $\begin{array}{c}\text { Speed } \\
(\mathrm{m} / \mathrm{s})\end{array}$ & $\begin{array}{l}\text { Trip } \\
(\mathrm{mm})\end{array}$ & $\mathbf{k}_{\mathbf{g}}$ & $(\mathrm{mm})$ & $k_{s} \quad(m m)$ & $\mathbf{k g}^{+}$ & $\mathbf{k}_{\mathrm{s}}^{+}$ & $\delta / k_{g}$ & $\mathbf{R e}_{\odot}$ \\
\hline & 30 & 9.5 & & 3 & 3.36 & 248 & 278 & 55 & 35200 \\
\hline \multirow[t]{5}{*}{ Present Study } & 30 & $2 \times 19$ & & 3 & 3.33 & 283 & 315 & 73 & 43500 \\
\hline & 60 & $2 \times 19$ & & 3 & 3.35 & 507 & 566 & 76 & 80200 \\
\hline & $\begin{array}{c}\text { Speed } \\
(\mathrm{m} / \mathrm{s})\end{array}$ & Surface & $\mathbf{k}_{\mathrm{g}}$ & $(\mathrm{mm})$ & $k_{s}(m m)$ & $\mathbf{k g}^{+}$ & $\mathbf{k}_{\mathrm{s}}^{+}$ & $\delta / k_{g}$ & $\mathbf{R e}_{\odot}$ \\
\hline & 38 & S-S & & 1.4 & 2.7 & 177 & 333 & 42 & 21200 \\
\hline & 50 & S-S & & 1.4 & 2.7 & 234 & 440 & 42 & 29800 \\
\hline \multirow[t]{4}{*}{ Blake 1970} & 38 & $D-L$ & & 2.3 & 4.9 & 311 & 648 & 26 & 21700 \\
\hline & 50 & D-L & & 2.3 & 4.9 & 412 & 861 & 26 & 28800 \\
\hline & 38 & D-S & & 1.4 & 1.9 & 172 & 224 & 41 & 21200 \\
\hline & 50 & D-S & & 1.4 & 1.9 & 227 & 296 & 41 & 28000 \\
\hline Aupperle \& & 50 & Grade 36 & & 0.5 & 2.5 & 81 & 405 & 56 & - \\
\hline \multirow[t]{6}{*}{ Lambert 1970} & 50 & Grade 12 & & 1.7 & 6.1 & 318 & 1141 & 20 & - \\
\hline & 50 & Grade 4 & & 3.8 & 11.9 & 858 & 2687 & 10 & - \\
\hline & 13 & 20 Grit(B) & & 0.95 & 1.60 & 57 & 95.2 & 63 & 1840 \\
\hline & 13 & 36 Grit (B) & & 0.53 & 1.30 & 31 & 76.5 & 40 & 1620 \\
\hline & 13 & 40 Grit & & 0.42 & 1.00 & 24 & 56.4 & 42 & 1560 \\
\hline & 13 & 60 Grit & & 0.26 & 0.44 & 14 & 23.9 & 43 & 1400 \\
\hline \multirow[t]{2}{*}{ Smith 2008* } & 13 & 60 Grit (B) & & 0.26 & 0.26 & 14 & 13.3 & 61 & 1390 \\
\hline & 13 & 100 Grit & & 0.14 & 0.07 & 7 & 3.0 & 206 & 1200 \\
\hline *Wall jet experiment. Values & 13 & 150 Grit & & 0.09 & 0.06 & 4 & 2.4 & 309 & 1100 \\
\hline presented computed directly & 13 & 180 Grit & & 0.08 & 0.04 & 4 & 1.8 & 296 & 1090 \\
\hline \multirow[t]{5}{*}{ from Smith's raw data } & 13 & 220 Grit & & 0.07 & 0.03 & 3 & 1.2 & 376 & 1040 \\
\hline & $\begin{array}{c}\text { Speed } \\
(\mathrm{m} / \mathrm{s})\end{array}$ & Surface & $\mathbf{k}_{\mathrm{g}}$ & $(\mathrm{mm})$ & d $(\mathrm{mm})$ & $\mathbf{k g}^{+}$ & $\mathbf{k}_{\mathrm{s}}^{+}$ & $\delta / \mathrm{k}_{\mathrm{g}}$ & $\mathbf{R e}_{\odot}$ \\
\hline & 27 & Fetch 1 & & 1 & 4 & - & - & 63.4 & - \\
\hline & 20 & Fetch 1 & & 1 & 4 & - & - & 64 & - \\
\hline & 27 & Fetch 2 & & 1 & 5.5 & - & - & 44.4 & - \\
\hline \multirow[t]{5}{*}{ Varano 2010} & 20 & Fetch 2 & & 1 & 5.5 & - & - & 44.5 & - \\
\hline & 27 & Fetch 3 & & 1 & 8 & - & - & 30.4 & - \\
\hline & 20 & Fetch 3 & & 1 & 8 & - & - & 48.6 & - \\
\hline & 27 & Fetch 4 & & 1 & 11 & - & - & 59.7 & - \\
\hline & 20 & Fetch 4 & & 1 & 11 & - & - & 53.2 & - \\
\hline
\end{tabular}




\subsubsection{Boundary Layer Turbulence}

The root-mean-square (RMS) turbulent velocity fluctuations were measured throughout the boundary layer over both smooth and rough walls using a quad-wire anemometer. The turbulence intensity profiles normalized with the friction velocity $u_{t}$ for the smooth and rough wall boundary layers can be found in Figure 3.7. The smooth wall $u^{\prime} / u_{t}$ turbulence intensities in the streamwise direction were found to closely match the results as found by Blake (1970), though they seem to have a somewhat slower turbulence decay as one moves away from the wall. The same trend appears for the vertical $\left(v^{\prime} / u_{t}\right)$ and spanwise $\left(w^{\prime} / u_{t}\right)$ component turbulent intensities, suggesting a slightly more turbulent freestream flow. Additionally, the present study shows $v^{\prime} / u_{t}$ values peaking at higher intensities near the wall when compared to Blake. The vertical component of the turbulent intensity peak has actually been found to be dependent on $R e_{\theta}$, with larger momentum thickness based Reynolds number flows peaking at higher intensities near the wall (Fernholz and Finley 1996).
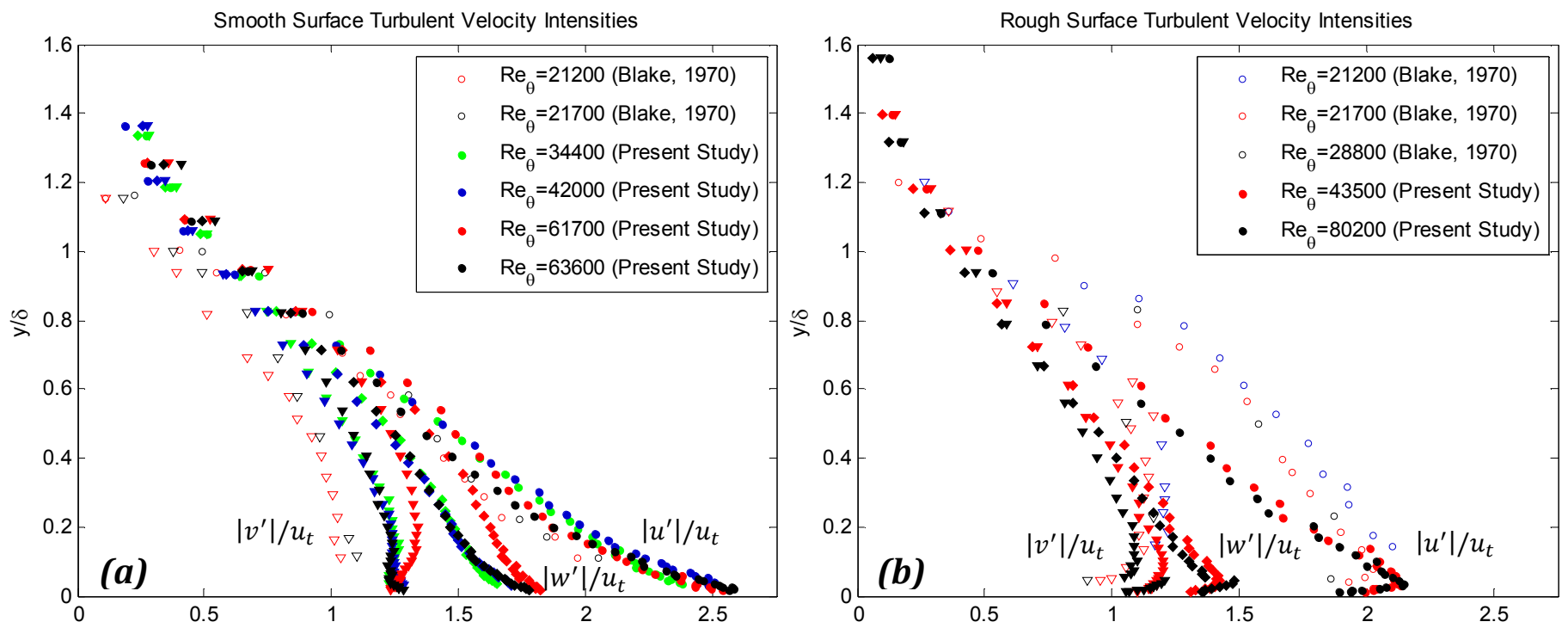

Figure 3.7. Boundary layer turbulence intensity profiles for (a) smooth and (b) rough walls.

The rough wall turbulence intensity profiles in Figure 3.7 show decreased turbulence levels near the surface for $u^{\prime}, v^{\prime}$, and $w^{\prime}$ due to the presence of the roughness elements. These values closely match what was found by Blake (1970) for values below $y / \delta=0.2$, though the streamwise and vertical turbulence levels were found to subsequently decrease more rapidly than Blake's findings. It is believed that the larger $\operatorname{Re}_{\theta}$ and $\delta / \mathrm{kg}_{\mathrm{g}}$ found in this study as listed in Table 3.3 are the primary factor for this more rapid decrease in turbulence intensities. The larger relative size of the boundary layer to the roughness height in this study $\left(\delta / k_{g}=73-76\right)$ as compared to that of Blake's $\left(\delta / k_{g}=26-42\right)$ would intuitively decrease the roughness's effects throughout the upper portions of the boundary layer. Consistent with this, it was found that the rough wall turbulent intensities above $y / \delta$ $=0.3$ had both a shape and slope that was more similar to what was observed over smooth walls. 
The turbulent kinetic energy (TKE) throughout the boundary layer is presented in Figure 3.8. While the normalized TKE at first appears to be proportionally lower over rough walls when compared to smooth walls, it should be noted that the skin friction coefficient, and thus friction velocity $u_{t}$, are both significantly higher over the rough surfaces. The strong effect of the roughness elements on the retardation of the boundary layer, especially near the wall, is evident in the sharp decrease in the TKE below about $0.05 \delta$. It should be restated here that velocity fluctuation profiles were taken at the center point equidistant between four roughness elements.

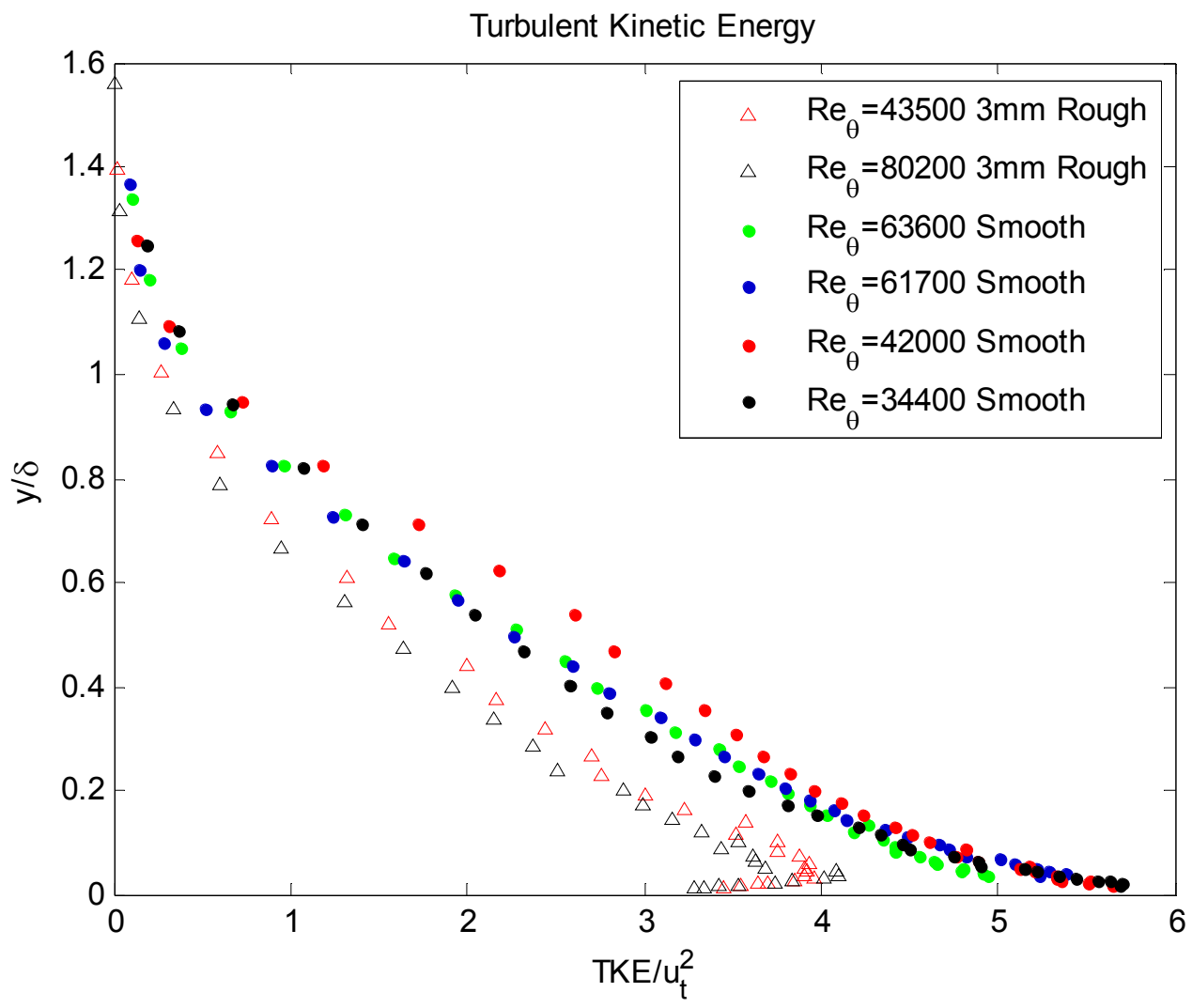

Figure 3.8. Boundary layer turbulent kinetic energy (TKE) comparison for rough and smooth surfaces. 

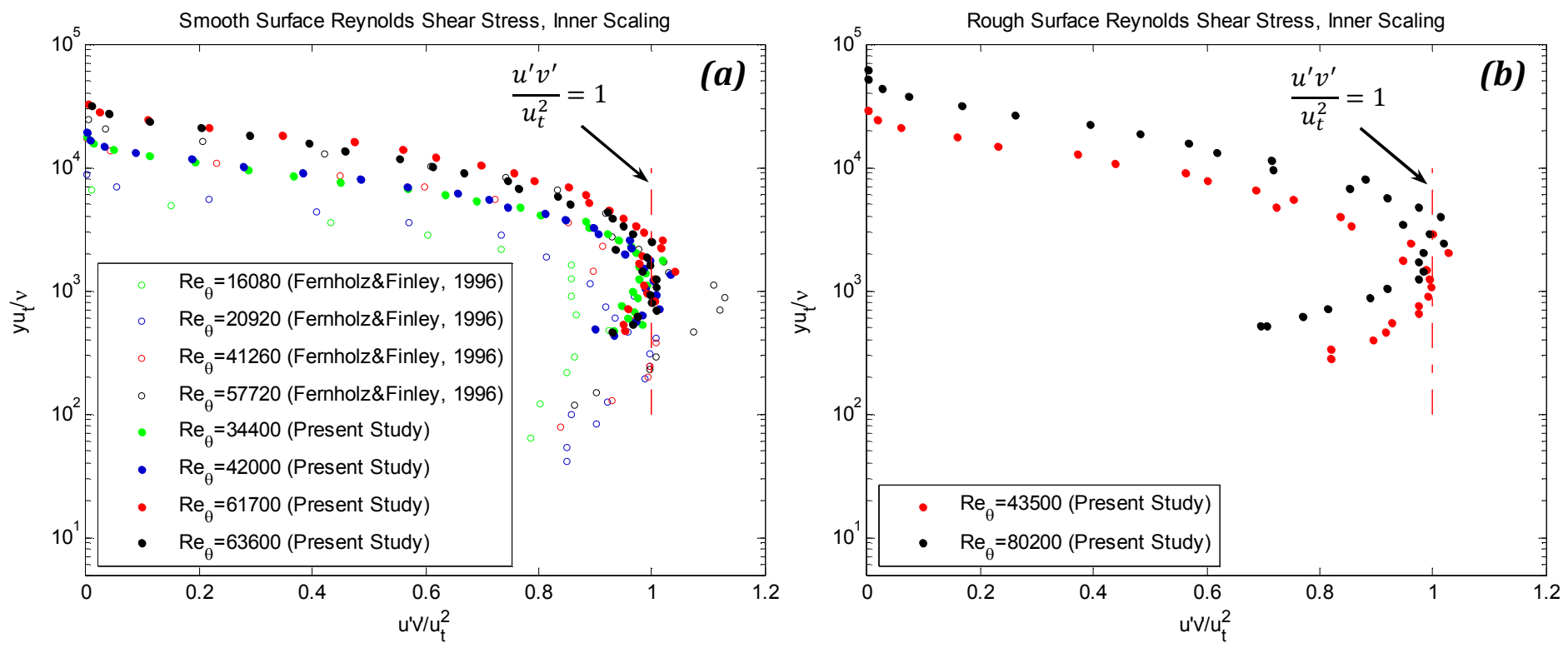

Figure 3.9. Boundary layer Reynolds shear stress profiles for (a) smooth and (b) rough walls.

The turbulent boundary layer Reynolds shear stress component $u^{\prime} v^{\prime}$ is related to wall shear as shown in Equation 3.9 (Schlichting 1979). When combining this with Equation 3.8, it can be shown that $u^{\prime} v^{\prime} / u_{t}^{2}$ should peak near the surface at a value of 1 . The peak of this Reynolds shear stress term has been shown to be an accurate way to determine the skin friction coefficient $C_{f}$ for both smooth and rough wall flows (Varano 2010). For this study, however, this relationship was just used as a verification of the law of the wall fitting method discussed in section 3.3.1.

$$
\tau_{w}=\rho u^{\prime} v^{\prime}
$$

Equation 3.9

In Figure 3.9, the normalized Reynolds shear stress $u^{\prime} v^{\prime}$ components for both smooth and rough surfaces are displayed. The data for the present study shows all $u^{\prime} v^{\prime} / u_{t}{ }^{2}$ components peaking near unity, consistent for correctly determined skin friction coefficient values and verifying the methods used in section 3.3.1. The vertical position of the Reynolds stress peak shows a strong dependence on $R e_{\theta}$ when the position was normalized on inner boundary layer scaling. Consistent with the results of Fernholz and Finley (1996), it was found that the location of the Reynolds stress peak increased with increasing $\operatorname{Re}_{\theta}$ for both smooth and rough walls. The comparison of the present study's smooth wall Reynolds shear stress profile at $R e_{\theta}=42000$ with that of Fernholz and Finley's review at $R e_{\theta}=41260$ shows a remarkably close agreement between the data sets.

\subsection{Surface Pressure Fluctuations}

\subsubsection{Surface Pressure Spectra}

Wall pressure fluctuations were measured along the false wall using six embedded B\&K type 4138 microphones aligned flush with the surface. Microphone wall positions were permuted to obtain a larger variety of separations, with one microphone being permanently offset in the spanwise direction to be used as a reference microphone for 
background acoustic cancellation when measuring the wall pressure spectra. The wall pressure spectral density $\Phi$ can be defined as the wall pressure spectrum $\phi_{p_{i} p_{i}}$ at a single point $i$, or in other words $\Phi(\omega)=\phi_{p_{i} p_{i}}(\omega, s)$ where $s=0$.

The wall pressure spectra measured over smooth walls for flow velocities between 22.4 and $67.2-\mathrm{m} / \mathrm{s}$ are shown in Figure 3.10, and the spectra measured over rough walls for flows between 20 and $60-\mathrm{m} / \mathrm{s}$ are shown in Figure 3.11. The wall pressure spectrum is displayed as a surface pressure level (SPL) normalized about a reference pressure of 20$\mu \mathrm{Pa}$ and shown in decibels for both the streamwise and spanwise microphone array locations. Rough wall spectra from spanwise microphone locations located directly behind roughness elements have been removed from Figure 3.11(b).

Inspection of Figure 3.10 and Figure 3.11 reveals many typical traits found in wall pressure spectra observed in other zero-pressure gradient turbulent boundary layer flows. A low signal to noise ratio limited the data found at very low frequencies, though there is clear evidence of a low frequency rise for each of the spectra before reaching a maximum. In the mid-frequency range after the spectral peak, a very broad and clearly defined linear overlap region is exhibited before the wall pressure spectra begin their sharp roll off at high frequencies. While an overlap region is commonly observed for most smooth wall flows and was expected to exist for rough wall flows, it is believed this is the first time a clearly defined overlap region for fully rough flow has actually been observed in a laboratory environment where outer region interference was not suspected. Also of note in these figures, the flow velocity effect of shifting spectral features towards higher frequencies is compounded with its effect of increasing the flow Reynolds number, which is known to broaden the mid-frequency overlap region (Goody 2004). As such, only a limited amount of the roll off region is shown for flow velocities greater than 33.6 and $30-\mathrm{m} / \mathrm{s}$ respectively for the smooth and rough wall flows.

In Figure 3.10 and Figure 3.11, the wall pressure spectra are shown to be almost invariant with spanwise and streamwise location for a fixed flow condition and Reynolds number. The boundary layer two-dimensionality and limited growth in this region, combined with this condition, strongly suggests a near homogeneous flow in planes parallel to the wall. As such, wall pressure spectral densities in later sections will be presented as the spectral averages across the microphone locations for fixed flow conditions. 

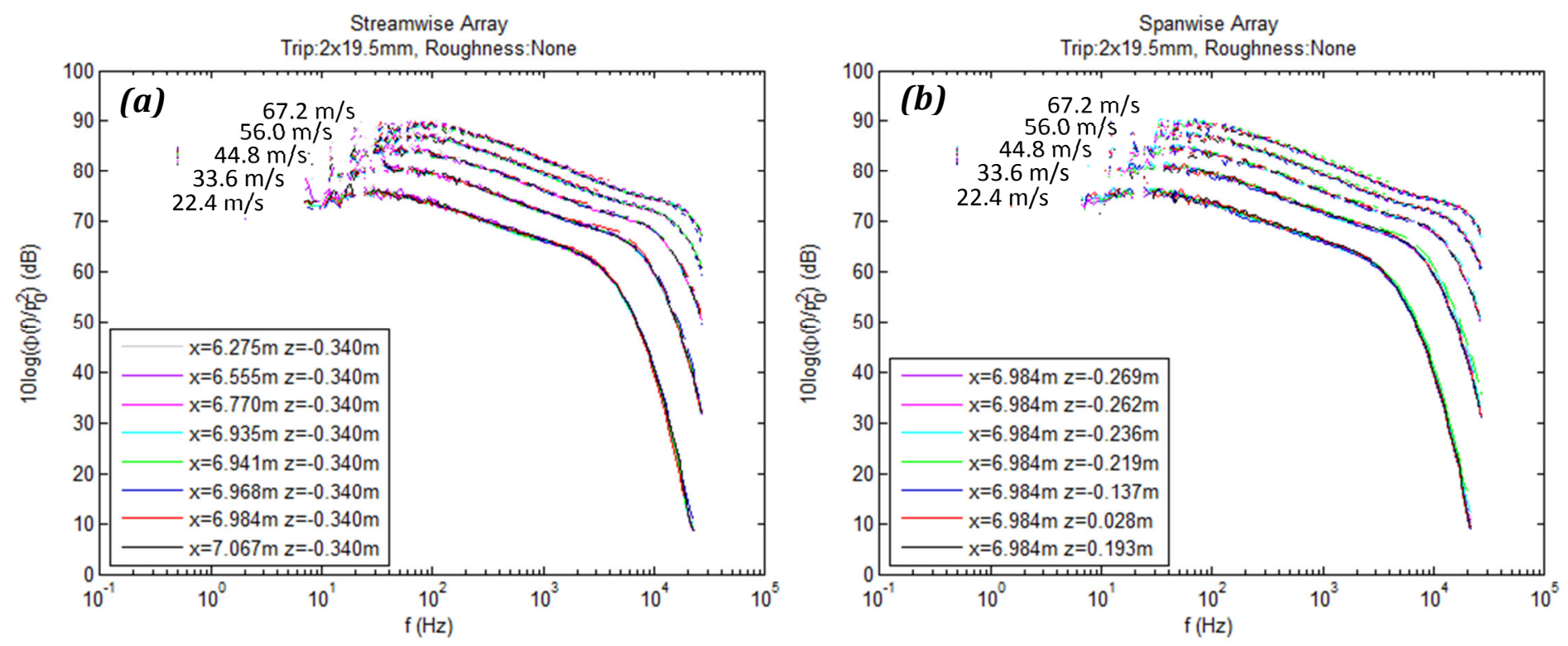

Figure 3.10. Wall pressure spectra for smooth walls using (a) streamwise and (b) spanwise microphone arrays.
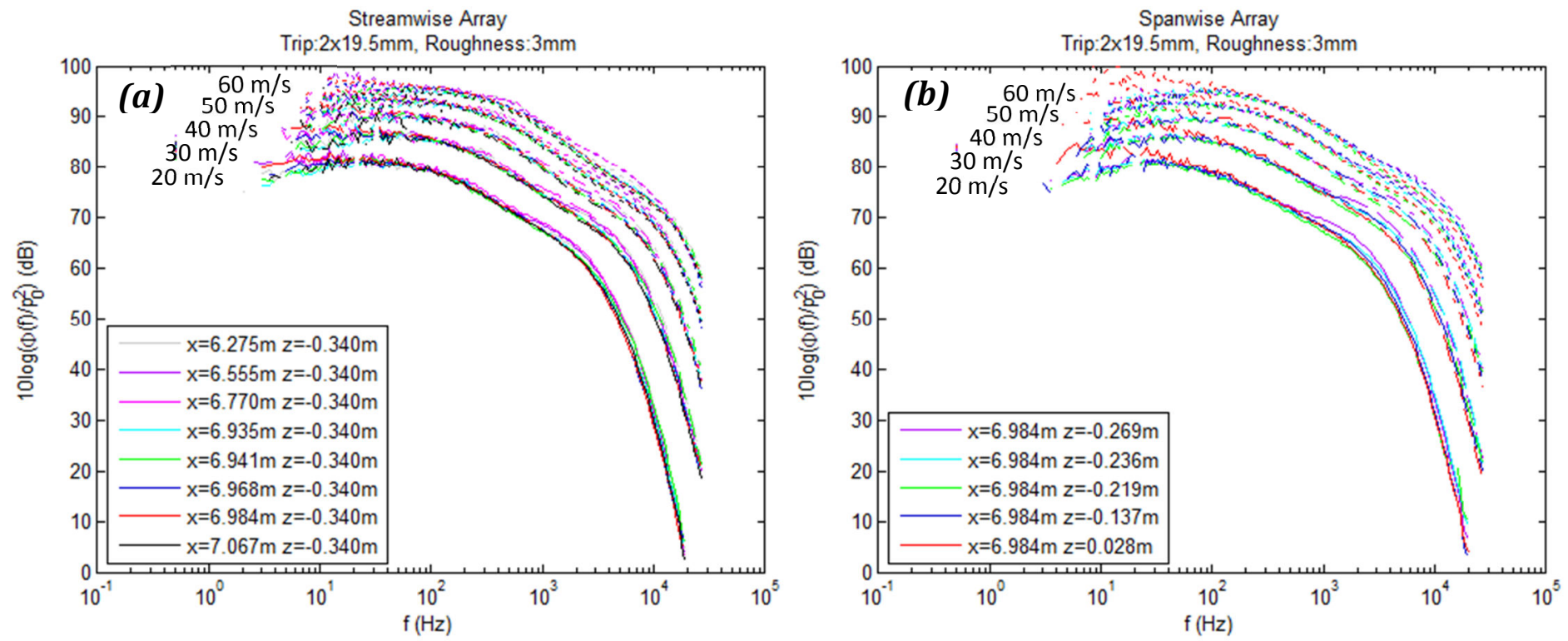

Figure 3.11. Wall pressure spectra for rough walls using (a) streamwise and (b) spanwise microphone arrays.

\subsubsection{Surface Pressure Spectral Scaling and Features over Smooth Walls}

The scaling behavior of the wall pressure spectrum can be used to link spectral features with their respective governing variables. At high frequencies, the roll off region has been associated with the inner portions of the boundary layer. The low frequency spectra, found just before the overlap region, has often been associated with the outer portions of the turbulent boundary layer, while the mid frequency region has been linked to the logarithmic layer in the boundary layer (Farabee and Casarella 1991, Goody 2004, Gravante et al. 1998). 
At high frequencies, it is generally accepted that the spectra collapse on the inner boundary layer variables $\Phi(\omega) u_{t}^{2} /\left(\tau_{w}^{2} v\right) v s . \omega v / u_{t}^{2}$ for smooth walls. The smooth wall pressure spectra scaled on these variables is shown in Figure 3.12 in comparison with the results from Farabee and Casarella (1991). A good collapse is shown along the high frequency roll off and the mid frequency overlap regions. The effect of Reynolds number on the lengthening of the overlap region can clearly be seen for these spectra. For each Reynolds number, the overlap region appears to have an upper limit near $\omega v / u_{t}^{2} \sim 0.4$ at high frequencies. It is found to extend down to $\omega v / u_{t}^{2}=0.002$ for $R e_{\theta}=76700$ in the present study, which is in stark contrast when compared to the length of the overlap region of the lowest Reynolds number found by Farabee and Casarella which ends at $\omega v / u_{t}^{2}=$ 0.05 for $R e_{\theta}=3386$.

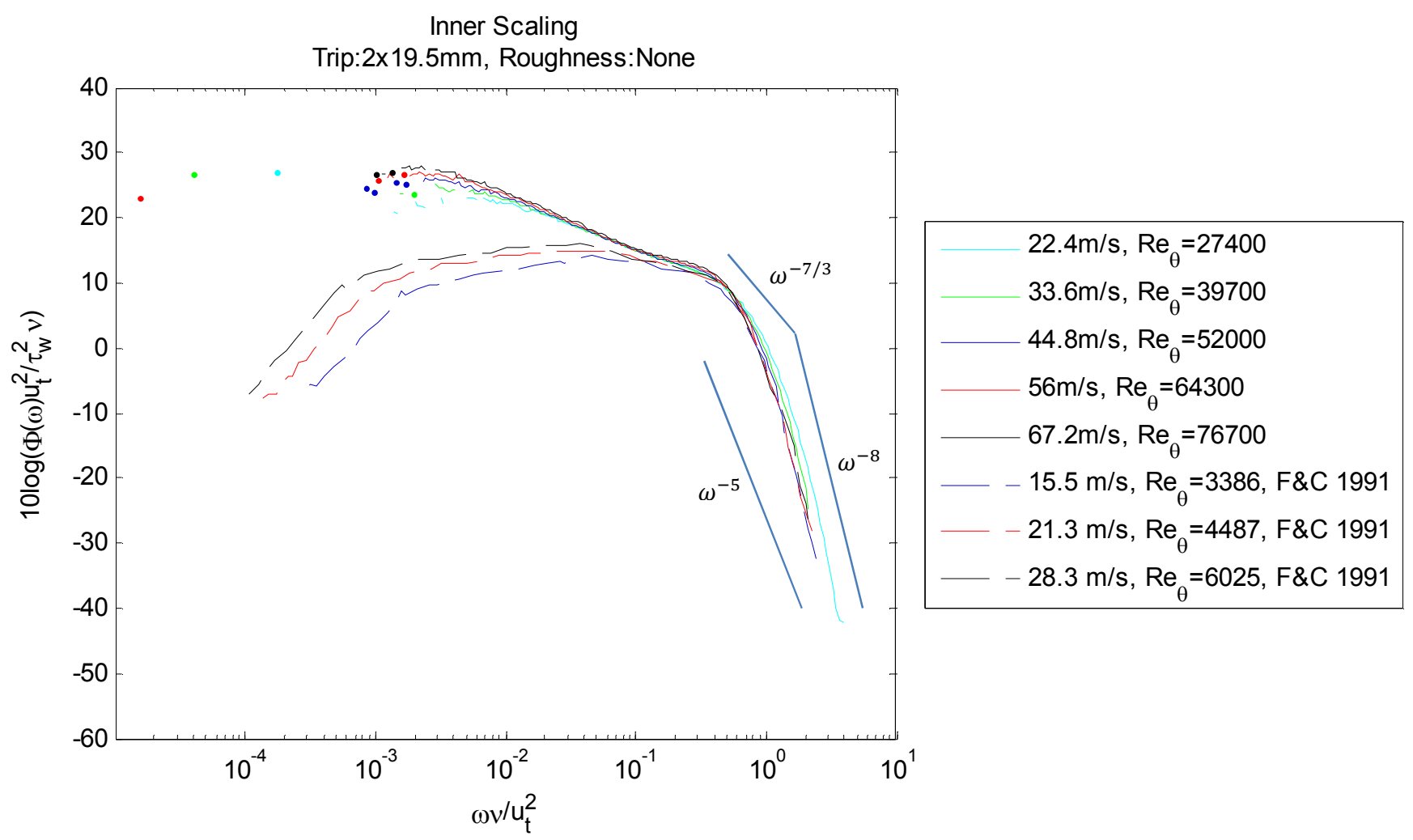

Figure 3.12. Wall pressure spectra for smooth walls normalized on inner variable scaling.

At frequencies just above the overlap region, it has been shown that there should be a small region with a $\omega^{-7 / 3}$ slope (Schewe 1983) which has been linked to pressure sources from the boundary layer buffer region (Gravante, et al. 1998). While somewhat difficult to assess the exact endpoints of this region in Figure 3.12, there is some evidence that this region exists in the present data between $\omega v / u_{t}^{2}=0.4$ and $\omega v / u_{t}^{2}=0.7$, which is in close agreement with Schewe's results showing the buffer region between $\omega v / u_{t}^{2}=0.4$ and $\omega v / u_{t}^{2}=0.8$. Beyond this buffer region at the highest frequencies of the roll off region, a $\omega^{-5}$ slope is typically expected (Goody 2004, Gravante et al. 1998). The present data, however, exhibits a larger slope closer to $\omega^{-8}$. This could be argued to be due to some spatial averaging over the $1 / 2-\mathrm{mm}$ pinhole for frequencies over $20-\mathrm{kHz}$, though this larger 
slope in the roll off region has been seen by other studies as well (Blake 1970, Farabee and Casarella 1991, Klewicki et al. 2008). Gravante et al. did find that insufficiently small microphone pinhole sizes with dimensionless diameters $d^{+}<18$ would show a more rapid high frequency roll off in the case of signal attenuation due to signal averaging. As was explained in section 2.5.2, some minor attenuation was expected to occur at high frequencies as the pinhole diameters $d^{+}$used in this study did not quite meet the requirements set by Gravante et al., though it is largely expected to affect the frequencies near and above $\omega v / u_{t}^{2}=1$. This conclusion is similar to that by Farabee and Casarella regarding their high frequency roll off.

At low to mid spectral frequencies, outer boundary layer variables are typically used to collapse the pressure spectra. The exact scaling factors used to do this, however, have been a topic of some debate. A classical scaling using $\Phi(\omega) u_{t} /\left(\tau_{w}^{2} \delta\right) v s . \omega \delta / u_{t}$ has been used by many researchers (Farabee and Casarella 1991, Goody 2004, Gravante et al. 1998), while a common early scaling of $\Phi(\omega) U_{e} /\left(q^{2} \delta^{*}\right) v s . \omega \delta^{*} / U_{e}$ utilized the dynamic pressure as the primary pressure scale. Additionally, a mixed scaling of $\Phi(\omega) U_{e} /\left(\tau_{w}^{2} \delta^{*}\right) v s . \omega \delta^{*} / U_{e}$ that combines elements of the previous two scales has also been prominently used (Blake 1970, Bradshaw 1967, Panton and Linebarger 1974). Farabee and Casarella (1991) reported that the scaling based on dynamic pressure $q$ only appeared to collapse the very low frequency spectra exhibiting a slope of $\omega^{2}$, a region not examined in the present study. Additionally, Goody (1999) reported a low to mid frequency spectral collapse when using numerous timescales, only as long as $\tau_{w}$ was used to collapse the pressure scale. As such, this study primarily focuses on the use of classical and mixed outer variable scaling.

The smooth wall pressure spectra normalized on classical and mixed outer variables is shown in Figure 3.13 in comparison with the smooth wall data from Blake (1970) and Farabee and Casarella (1991). The present results agree favorably with those of the other studies, showing an excellent collapse along the low frequency and overlap regions. The maximum spectral peak found between the low frequency region and the overlap region was observed for the present data to be near $\omega \delta / u_{t} \cong 58$, which is in general agreement with the other findings when normalized on the classical outer variables. However, when using mixed outer variable scaling, the spectral peak was found to be at a somewhat lower normalized frequency than what was seen by the other results. The spectral peaks seem to show good agreement within their respective studies, with only a small discrepancy being shown between the different experiments. The difference thus could be more of a result from the error associated with the different methods used in obtaining the displacement thickness $\delta^{*}$, though the more reliable nature of the classical variable scaling corresponds well with the claims of Bull (1996) and Farabee and Casarella (1991).

A clear overlap region is shown in the mid frequency range in Figure 3.12 and Figure 3.13, with the region shown to collapse when using both inner and outer variable scaling. This region has been linked with the turbulence activity located in the logarithmic region of the boundary layer (Farabee and Casarella 1991, Gravante et al. 1998), with a frequency width dependent on Reynolds number. It has previously been theorized that this region should exhibit a $\omega^{-1}$ dependency (Bradshaw 1967, Panton and Linebarger 1974) though experimentally this is seldom seen as is the case in the present study where a $\omega^{-0.83}$ slope is observed. Many other experimental results also show the overlap region exhibiting between a $\omega^{-0.7}$ and a $\omega^{-0.83}$ dependency (Blake 1970, Farabee and Casarella 1991, Goody 2004, McGrath and Simpson 1987), with only a few suggesting a $\omega^{-1}$ dependency (Gravante 
et al. 1998, Klewicki et al. 2008). Often experimental studies not seeing a $\omega^{-1}$ slope in the overlap region would attribute it to not achieving large enough Reynolds numbers, though the large Reynolds numbers in the present study and lack of a $\omega^{-1}$ slope would suggest that perhaps this is not the case. Additionally, a closer inspection of some of the results that do suggest a $\omega^{-1}$ dependency presents the possibility that there may be other influencing factors. Reexamination of wall pressure spectra presented by Gravante et al. (1998), suggests that an overlap region slope of $\omega^{-0.83}$ could also be interpreted from the data, though low frequency noise makes the measurement of the slope difficult to define. In Klewicki et al. (2008), the surface pressure spectra were measured over the salt flats of Utah. In this study, the overlap region was also reported to have a $\omega^{-1}$ dependency, an effect at the time believed to be reached due to the extremely large Reynolds numbers achieved in the study. Reexamination of the data does in fact support a larger $\omega^{-1}$ dependence in the overlap region, with indications that a slope as large as $\omega^{-1.08}$ could also be interpreted from the data. However, results from the present study discussed in section 3.4.3 suggest that the larger slope found in the overlap region in Klewicki et al.'s data may be more due to the flow residing in the transitionally rough regime than the large Reynolds numbers achieved.

To fully understand why the theoretical dependence of the overlap region does not correspond with what is often seen experimentally, it is useful to re-derive the dependency. Some of the early reasoning for the existence of an overlap region focused on the relationship between classical outer and inner variable scaling (Panton and Linebarger 1974). Following this reasoning for a zero-pressure gradient flow, one can define the spectrum scaled on outer variables as $P$ and the pressure spectrum scaled on inner variables as $Q$ as shown in Equation 3.10.

$$
P\left(\frac{\omega \delta}{u_{t}}\right)=\frac{\Phi(\omega) u_{t}}{\tau_{w}^{2} \delta} \quad \text { and } \quad Q\left(\frac{\omega v}{u_{t}^{2}}\right)=\frac{\Phi(\omega) u_{t}^{2}}{\tau_{w}^{2} v}
$$

Thus if an overlap region existed over a range of frequencies where both inner and outer variable scaling would work, then Equation 3.11 would hold true over this range, which may also be simplified to Equation 3.12.

$$
\begin{gathered}
\frac{\tau_{w}^{2} \delta}{u_{t}} P\left(\frac{\omega \delta}{u_{t}}\right)=\frac{\tau_{w}^{2} v}{u_{t}^{2}} Q\left(\frac{\omega v}{u_{t}^{2}}\right) \\
\delta P\left(\frac{\omega \delta}{u_{t}}\right)=\frac{v}{u_{t}} Q\left(\frac{\omega v}{u_{t}^{2}}\right)
\end{gathered}
$$

Using this classical scaling, it is typically claimed at this point that the boundary layer thickness $\delta$ is independent of kinematic viscosity $v$ and friction velocity $u_{t}$. Using this argument it could thus be claimed that the pressure spectra should behave as an inverse function of $\omega \delta$ when normalized on outer variables and an inverse function of $v / u_{t}$ when normalized on inner variables. Additionally, knowing that the logarithmic region behaves linearly on a logarithmic plot, one could express the pressure spectra in the overlap region of the form seen in Equation 3.13 where $A$ and $B$ are constants. Note, however, that this 
reasoning breaks down when using mixed outer boundary layer variables, as $\delta^{*}$ is not independent of $u_{t}$.

$$
P\left(\frac{\omega \delta}{u_{t}}\right) \sim A(\omega \delta)^{-a} \quad \text { and } \quad Q\left(\frac{\omega v}{u_{t}^{2}}\right) \sim B\left(\frac{\omega v}{u_{t}}\right)^{-a} \quad \text { Equation } 3.13
$$

Knowing this relationship, substitution of Equation 3.13 into Equation 3.12 results in Equation 3.14. This can be rearranged to solve for the ratio of the constants as seen in Equation 3.15.

$$
\begin{aligned}
& \delta A(\omega \delta)^{-a}=\frac{v}{u_{t}} B\left(\frac{\omega \nu}{u_{t}}\right)^{-a} \\
& \frac{A}{B}=\left(\frac{\delta u_{t}}{v}\right)^{a-1}=\left(R e_{\delta}\right)^{a-1}
\end{aligned}
$$

Only if $a=1$ can Equation 3.15 be consistent with the presumed functional dependence in Equation 3.10. This realization indicates an $\omega^{-1}$ dependency in Equation 3.14, as was the conclusion by Panton and Linebarger (1974). As this is not the case found experimentally, one could contend that some of the assumptions made in this argument are incorrect. Specifically, one could argue that using the outer variable mixed scaling would preclude a $\omega^{-1}$ dependency in the overlap region as $\delta^{*}$ is not independent of $u_{t}$. Bradshaw (1967) proposes that a $\omega^{-0.83}$ dependency found experimentally could actually support a $k^{-1}$ wavenumber dependency ${ }^{2}$ due to the normalized convection velocity $U_{c} / U_{e}$ being less than one and dependent on frequency. However it is argued convincingly by Panton and Linebarger that since the pressure fluctuations are represented as a spectral density then the relationship between $\omega$ and $k$ has no impact on the spectral shape and a -1 slope should be seen regardless.

Another factor that could be preventing the theorized $\omega^{-1}$ dependency regards the structure and makeup of the turbulent boundary layer. Evidence was presented in Morrison et al. (1992) that the turbulence structures in the outer portions of the boundary layer were more freely moving than formerly thought and thus not as strictly segregated to their respective boundary layer heights as previous theories involving attached wall eddies had suggested. In Long and Chen (1981) and Wosnik et al. (2000) evidence of a mesoregion in the boundary layer is shown to exist that would imply a viscous influence much further beyond the viscous sublayer than classical boundary layer theory would suggest. As such, Morrison (2007) proposed that even as the flow Reynolds number approached infinity, large scale eddies would never fully decouple from their viscous effects. This realization would imply that many of the assumptions used in deriving the theoretical $\omega^{-1}$ dependency of the wall pressure spectra overlap region would, of course, be invalid.

\footnotetext{
2 Initial theory by Bradshaw (1967) actually suggested a $k^{-1}$ overlap dependency, not specifically a $\omega^{-1}$ relationship.
} 
Outer Scaling

Trip:2x19.5mm, Roughness:None

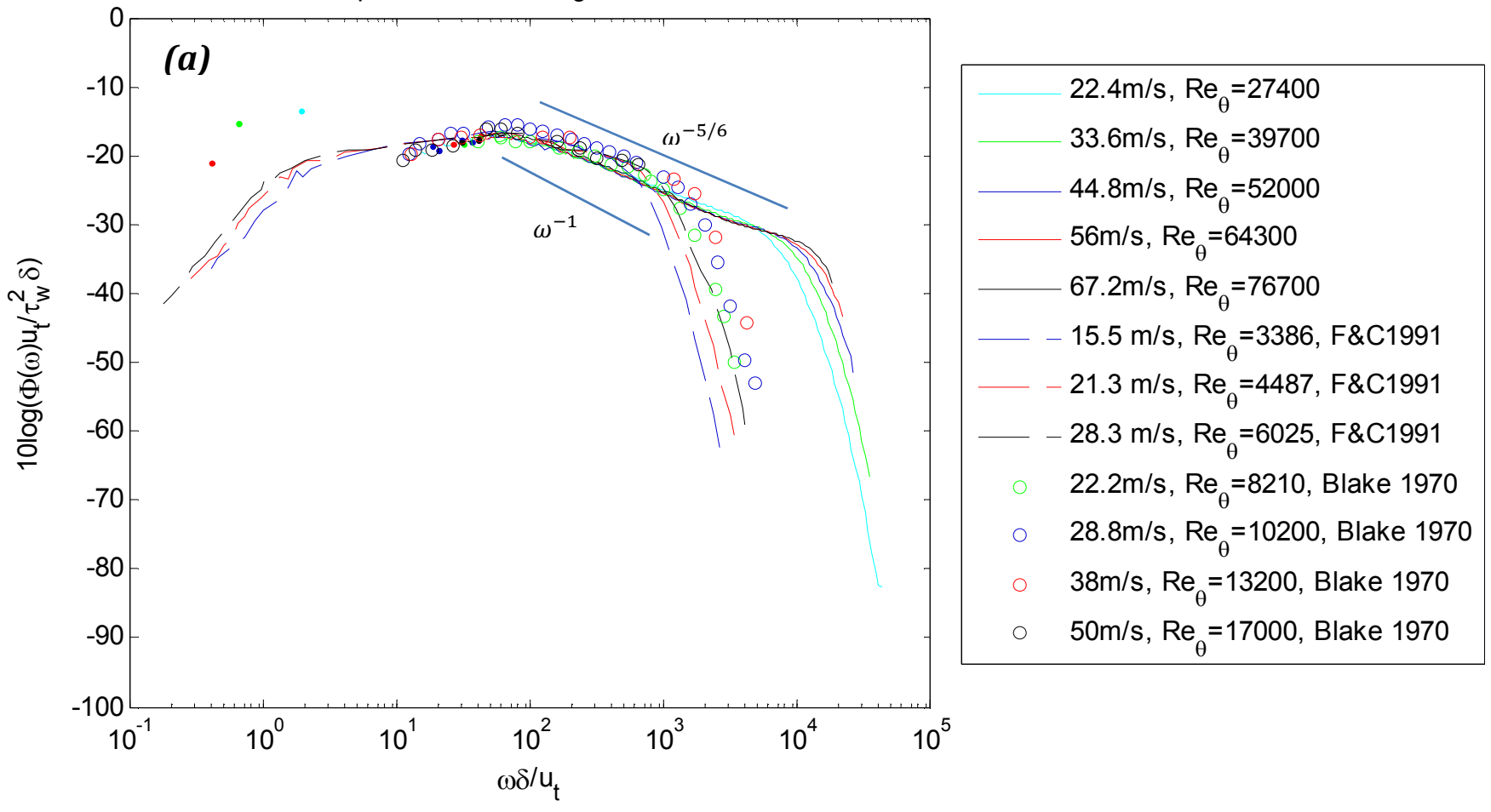

Outer Scaling

Trip:2x19.5mm, Roughness:None

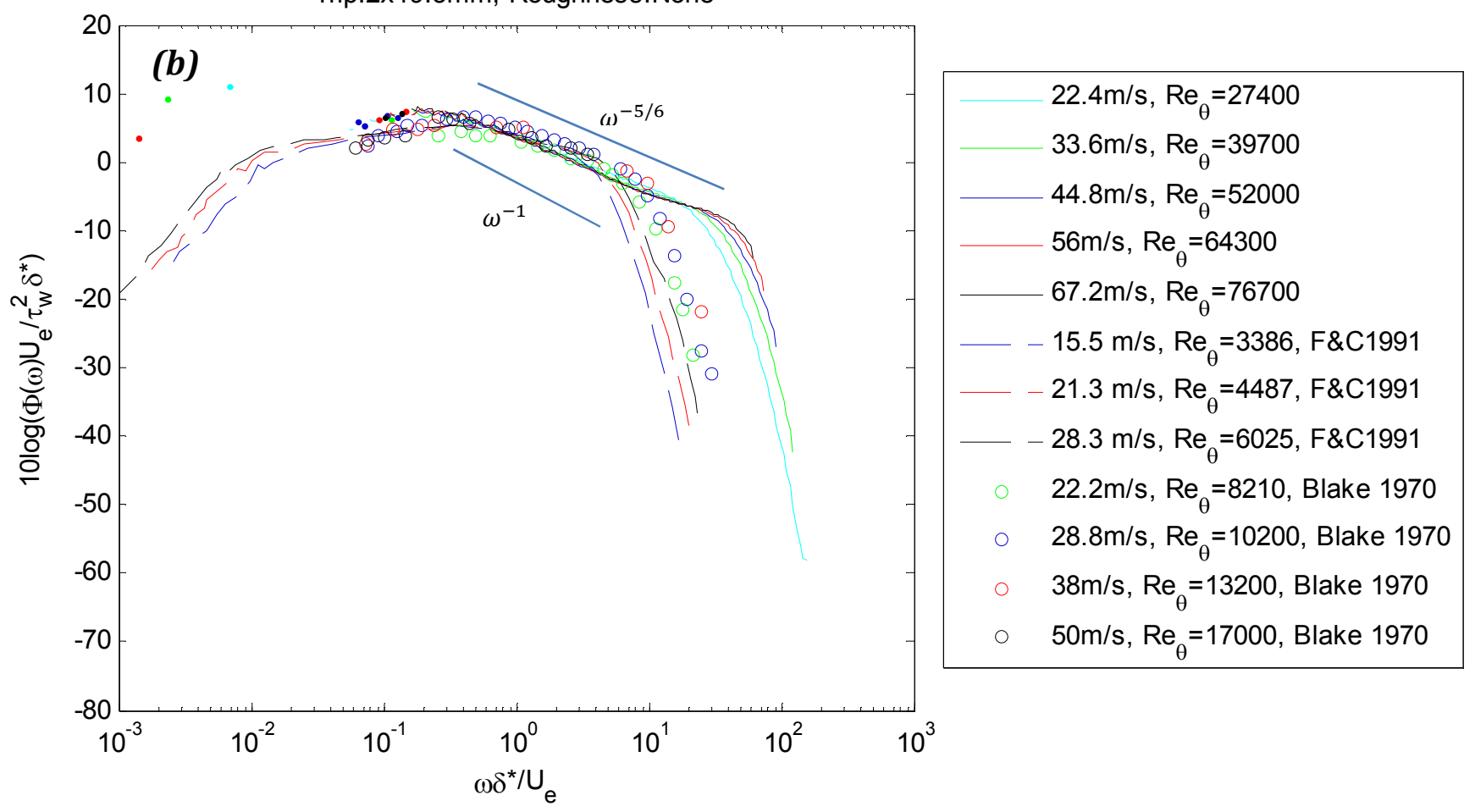

Figure 3.13. Wall pressure spectra for smooth walls normalized on (a) classical outer variable scaling and (b) mixed outer variable scaling. 


\subsubsection{Surface Pressure Spectral Scaling and Features over Rough Walls}

For fully rough flows, roughness has the effect of destroying the laminar sublayer and viscous cycle present in the buffer layer (Jiménez 2004). This has the effect of causing the mean flow to lose its dependence on viscosity, though it is unclear on whether the wall pressure fluctuations also lose their viscous dependence. Due to the turbulent flow and roughness element interaction, smooth wall inner variable scaling will not collapse the rough wall spectra.

Blake (1970) proposed the roughness based inner scaling $\Phi(\omega) u_{t} /\left(\tau_{w}^{2} k_{g}\right) v s . \omega k_{g} /$ $u_{t}$ to be used to collapse the rough wall pressure spectra at high frequencies. It was argued that the difference between the smooth and rough wall pressure spectra normalized on outer variables could be related to the Strouhal number $\omega k_{g} / u_{t}$, which was used as the frequency scale. It was admitted that this roughness based inner scaling did not account for roughness element spacing or sparseness, though was argued later (Blake 1971) to be less empirical than the only other roughness based inner scaling model at the time (Aupperle and Lambert 1970) while still obtaining a better level of collapse at high frequencies. Between the roughness based inner variable scales proposed in Blake (1970), Aupperle and Lambert (1970), and Smith (2008), only Blake's scaling has been shown to consistently collapse the rough wall pressure spectra in multiple studies. Up until this point however, a large range of pressure spectra for fully rough flows has not been compiled while using this scaling. Previous researchers have often just applied Blake's scaling over a narrow range of experimental conditions and Reynolds numbers.

The rough wall pressure spectra for fully rough flows scaled on Blake's (1970) roughness based inner variable scaling is shown in Figure 3.14. Note that Smith's (2008) data was taken in a wall jet tunnel such that only the high frequency spectra should be comparable to the other studies. From this figure, Blake's proposed scaling does not appear to collapse the data when a wide range of fully rough flow conditions are compared. It should be noted that Blake, Smith and Varano all found a moderate collapse for their individual data when using Blake's scaling, which is still apparent in Figure 3.14. The data from each of these individual studies does appear to show a moderate collapse on themselves, though it is believed that this is due to each of these studies only having been taken over a limited range of Reynolds numbers while using at least partly similar roughness configurations. While Blake did notice some difficulties with his scaling when collapsing roughness of both different sparseness and size, it was contended that differences at high frequencies were most likely due to unequal roughness element spacing. The results from the present study, however, do not support this. In the present study all flows utilized the same roughness fetch and element spacing, though they still appear to have some variation. Specifically, this scaling appears to exhibit a Reynolds number dependence.

Recently, Varano (2010) proposed another roughness based inner variable scaling. Based off Blake's roughness scaling, Varano explained that for fully rough flows, friction velocity should be proportional to the edge velocity. If replaced, one obtains the scaling $\Phi(\omega) /\left(\rho^{2} U_{e}^{3} k_{g}\right) v s . \omega k_{g} / U_{e}$. This scaling is applied in Figure 3.15. Note that due to many experiments not reporting flow density, only the present results and Varano's data are shown. A collapse very similar to what was found using Blake's scaling is observed as would be expected. 


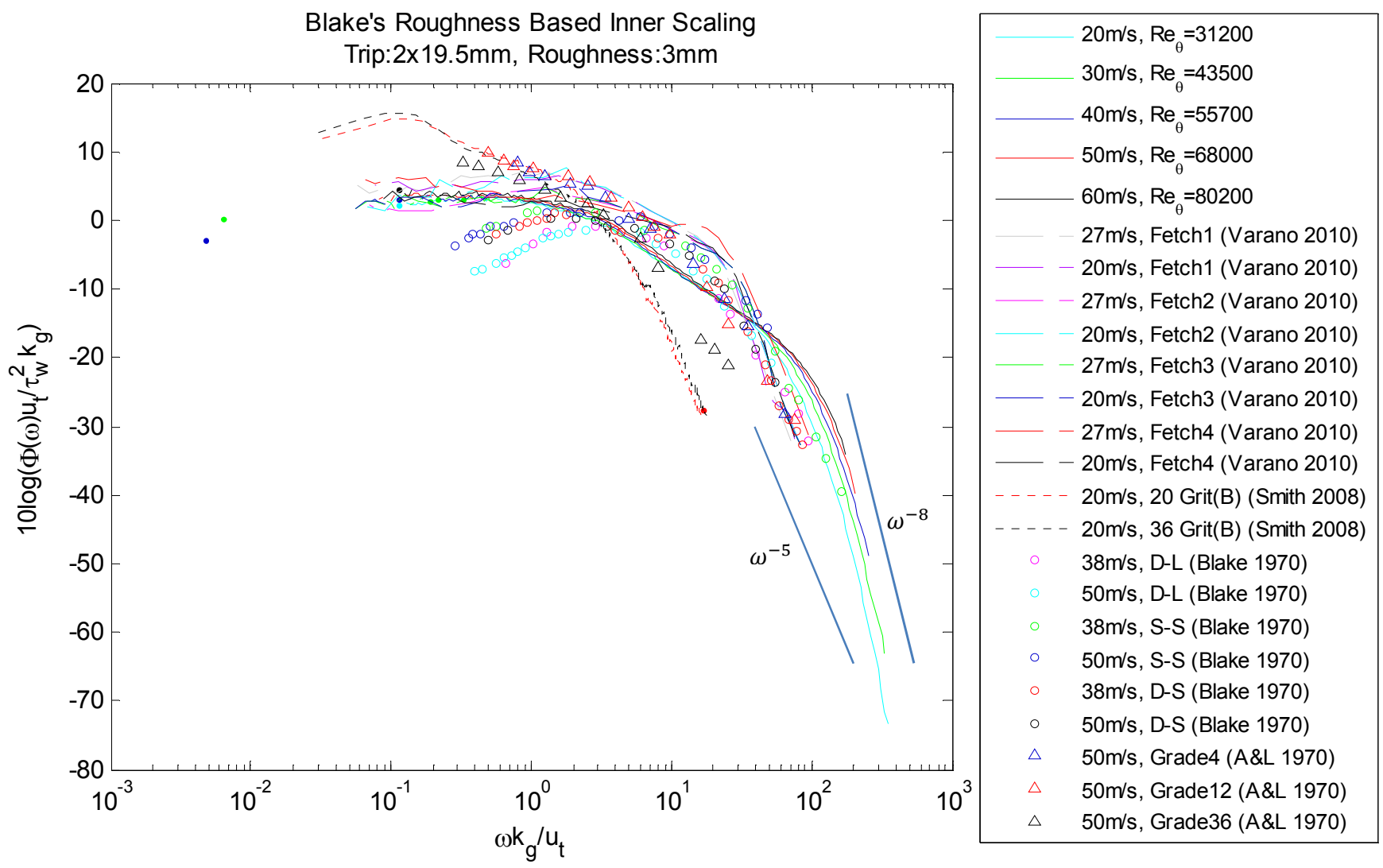

Figure 3.14. Wall pressure spectra for rough walls normalized on Blake's (1970) roughness based inner variable scaling.

To help determine a more universal inner variable roughness based scaling, smooth wall inner variable and a modified version of Blake's variables were used to scale the rough wall pressure spectra. Blake's scaling was modified by replacing the geometric roughness height $k_{g}$ with the equivalent sand grain roughness height $k_{s}$ in an attempt to account for roughness element spacing. The results of both of these scaling as applied to the present study's data are shown in Figure 3.16. It is apparent from this figure that while neither set of scaling variables collapse the data at high frequencies, they do appear to exhibit an inverse relationship with each other for increasing Reynolds number flows.

The difference between the inner scaling variables shown in Figure 3.16 stems solely from the particular timescales used. The timescale for the wall bounded flow is $v / u_{t}^{2}$, while $k_{S} / u_{t}$ is associated with the timescales for the roughness elements. It could be assumed that even for fully rough flows, both timescales may still influence the near wall turbulent structures. A diagram of what this situation would look like is shown in Figure 3.17. Note that the arrows in this figure do not denote distances, but rather where the timescales would be imagined to be located. Specifically, it would be expected that the wall bounded time scales would be associated with the relative spacing between roughness elements, while the roughness timescale would be expected to be associated with the equivalent roughness height. 


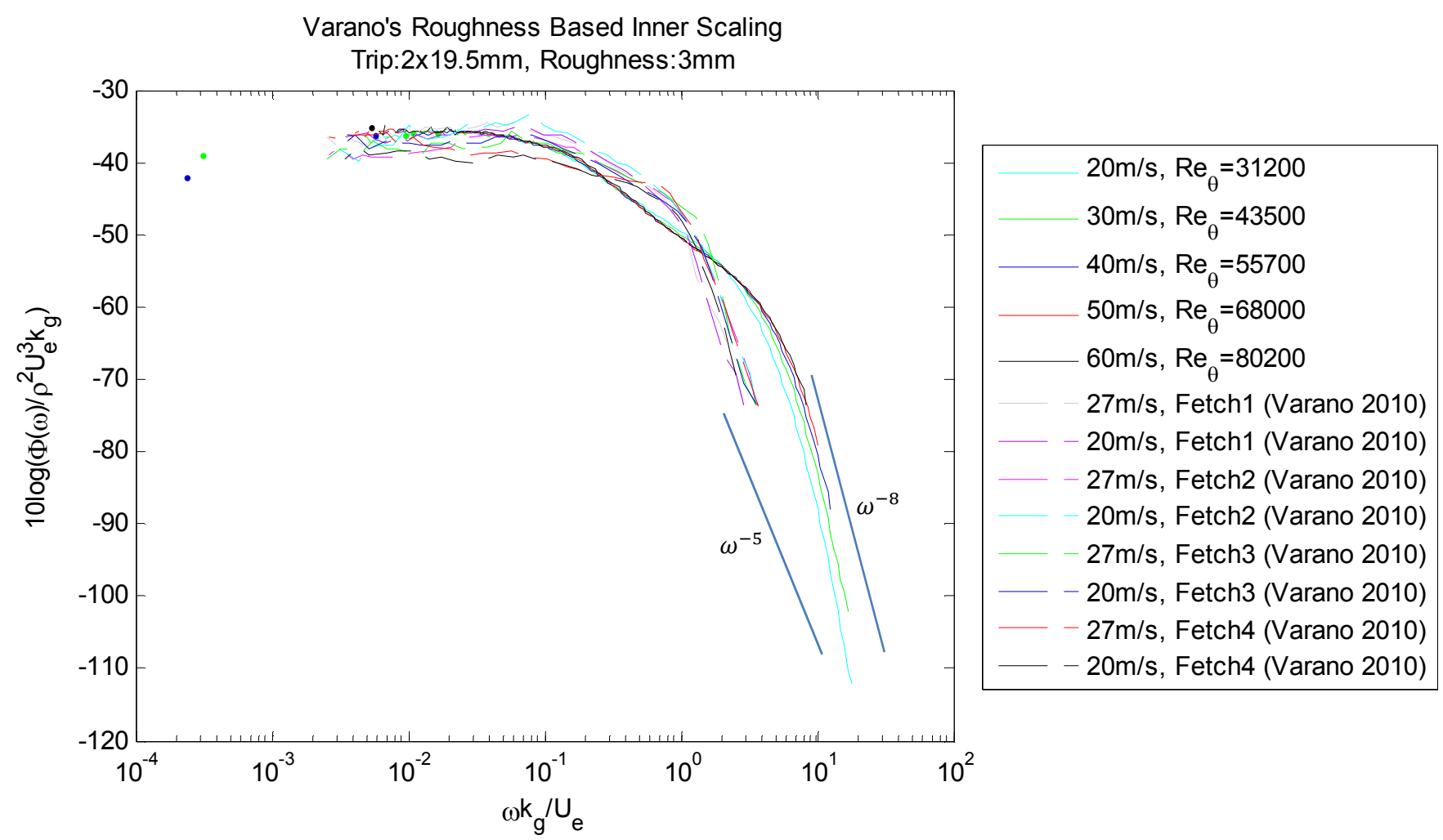

Figure 3.15. Wall pressure spectra for rough walls normalized on Varano's (2010) fully rough flow inner variable scaling.
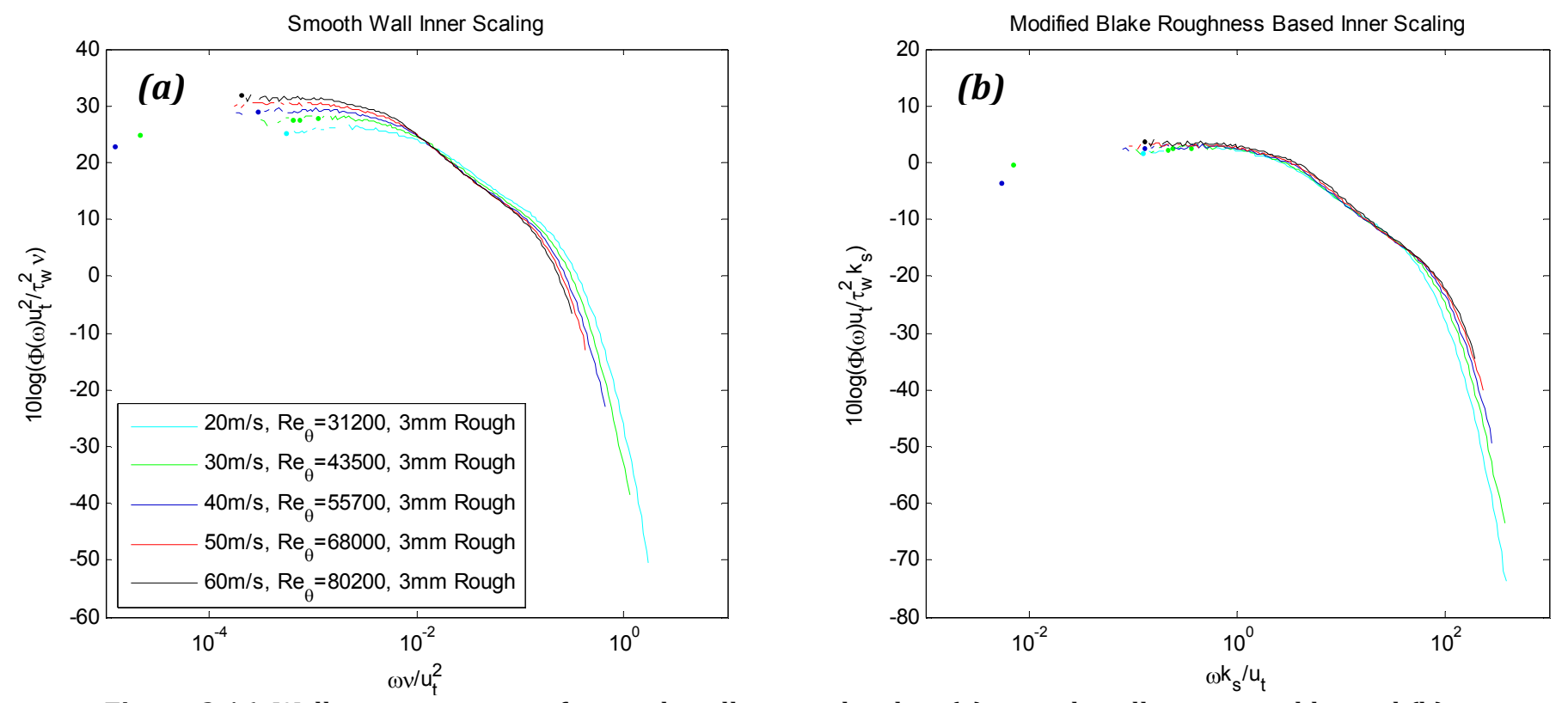

Figure 3.16. Wall pressure spectra for rough walls normalized on (a) smooth wall inner variables and (b) a modified Blake rough wall inner variable scaling.

From Figure 3.17 one can see that with a more sparsely spaced roughness configuration expected to produce a lower value of $k_{s}$, the relative ratio of the wall scale would become larger when compared to the roughness scale. Conversely, this diagram 
would imply that with larger equivalent roughness heights the roughness scaling would become dominant. Additionally, if the flow velocity were to increase, the friction velocity should also proportionally increase. As this occurs, the timescales associated with the roughness elements would become dominant compared to that of the wall scaling, an effect that would be expected.

As these behaviors appear to make physical sense, a meshing of time scales is needed. It is assumed that $k_{s}$ will function as a regulator between the ratio of the wall and roughness timescales, and that both timescales will be simultaneously affecting the wall pressure field. Using these assumptions, a simple geometric mean of the two timescales can be found as seen in Equation 3.16. Application of this timescale would result in the roughness based inner variable scaling $\Phi(\omega) u_{t}^{2} /\left[\tau_{w}^{2} v\left(k_{s}^{+}\right)^{0.5}\right] v s . \omega v\left(k_{s}^{+}\right)^{0.5} / u_{t}^{2}$, or equivalently $\Phi(\omega) u_{t}^{1.5} /\left(\tau_{w}^{2} v^{0.5} k_{s}^{0.5}\right) v s . \omega v^{0.5} k_{s}^{0.5} / u_{t}^{1.5}$.

$$
\sqrt{\frac{v}{u_{t}^{2}} \frac{k_{s}}{u_{t}}}=\frac{\left(k_{s} v\right)^{0.5}}{u_{t}^{1.5}}=\frac{v}{u_{t}^{2}}\left(k_{s}^{+}\right)^{0.5}
$$

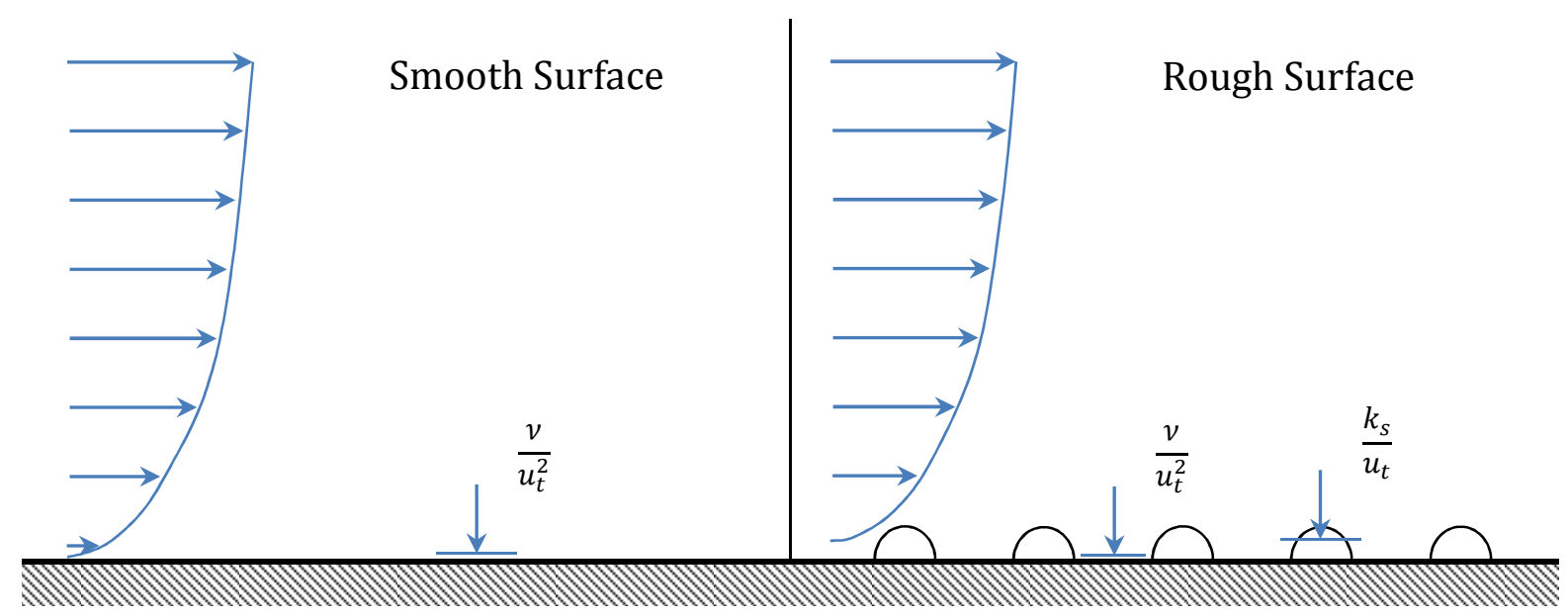

Figure 3.17. Wall pressure inner scaling variable location diagram. Objects are not to scale.

The rough wall pressure spectra scaled on this proposed roughness based inner variable scaling is shown in Figure 3.18(a) for only the present study so that a clearer display of the data collapse can be observed. Figure 3.18(b) shows the proposed scaling along with the scaled results from Blake (1970), Aupperle and Lambert (1970) and Smith (2008). Results from Varano (2010) could not be compared as no $k_{s}$ values were listed. A much more complete collapse is seen by the data in the high frequency roll off region as would be expected for inner variable scaling. While the overlap region appears to scale well between similar roughness configurations, it does not appear to show the same level of collapse as is seen at high frequencies between spectra of different roughness configurations. It is worth noting that this scaling produces an overlap region collapse between different roughness configurations very similar to what was exhibited by Blake's roughness based inner scaling. As is discussed later in this section, a spectral collapse along the overlap region may not even be possible for different roughness types. It is believed that the overlap region slope may not always be a fixed value as it is for the smooth wall 
spectra, but rather a function of the roughness configuration. Despite this, the high frequency collapse shown in Figure 3.18 would suggest that at least in the inner most portions of the boundary layer, both the wall bounded timescales and the roughness timescales influence the turbulence structures contributing to the wall pressure spectra. Interestingly, this scaling implies that flow viscosity still has an effect on the wall pressure field and turbulent structure generation over fully rough flows.

The viscosity dependence that is implied by this scaling is in contrast with the typical inner boundary layer scaling used for mean flow velocities over rough walls where the mean velocity is assumed to be independent of viscosity. When considering this scaling implication however, a few considerations should be noted. Over fully rough walls, viscous independence is generally thought of as being applied to the mean velocity over the rough walls. The rough wall pressure spectrum is a measure of the integrated boundary layer effects, including the flow that can occur between the roughness elements. In the narrow gaps between the roughness elements, especially for discretely spaced roughness configurations, the flow may behave similarly to a smooth wall over very short distances since no roughness elements interfere with the fluid in these narrow gaps. Even over more distributed roughness configurations such as those found using sand grains or sandpaper, smaller valleys can still be found between the larger roughness elements. Even if smaller roughness elements are located in these valleys, it could be argued that the interstitial flow inside of these valleys would be locally transitionally rough, and thus still dependent on viscosity. As one moves into higher roughness regimes where these small transitionally rough valleys would increasingly become relatively small, the $k_{s} / u_{t}$ term would become more dominant resulting in a corresponding decreased emphasis on viscosity dependence. Finally, it can be observed that the wall pressure spectrum is largely dependent on the turbulent velocities, with the mean flow primarily affecting the turbulent convective velocities. This would indicate that while the mean flow itself may as a whole become independent of viscosity, turbulent structure generation near the wall, including the spaces between the roughness element valleys, would remain dependent on viscosity even for fully rough flows.

In Figure 3.18, the high frequency slope along the roll off region was found to be near $\omega^{-8}$, though it is again this is believed to be due to some minor spatial averaging over the $1 / 2-m m$ pinhole due to insufficiently small dimensionless pinhole diameters $d^{+}$. It should be noted, however, that the rough surface's large interference with the inner regions of the boundary layer could suggest that a $\omega^{-5}$ slope in the roll off region may not necessarily be expected. 
Proposed Roughness Based Inner Scaling

Trip:2x19.5mm, Roughness:3mm

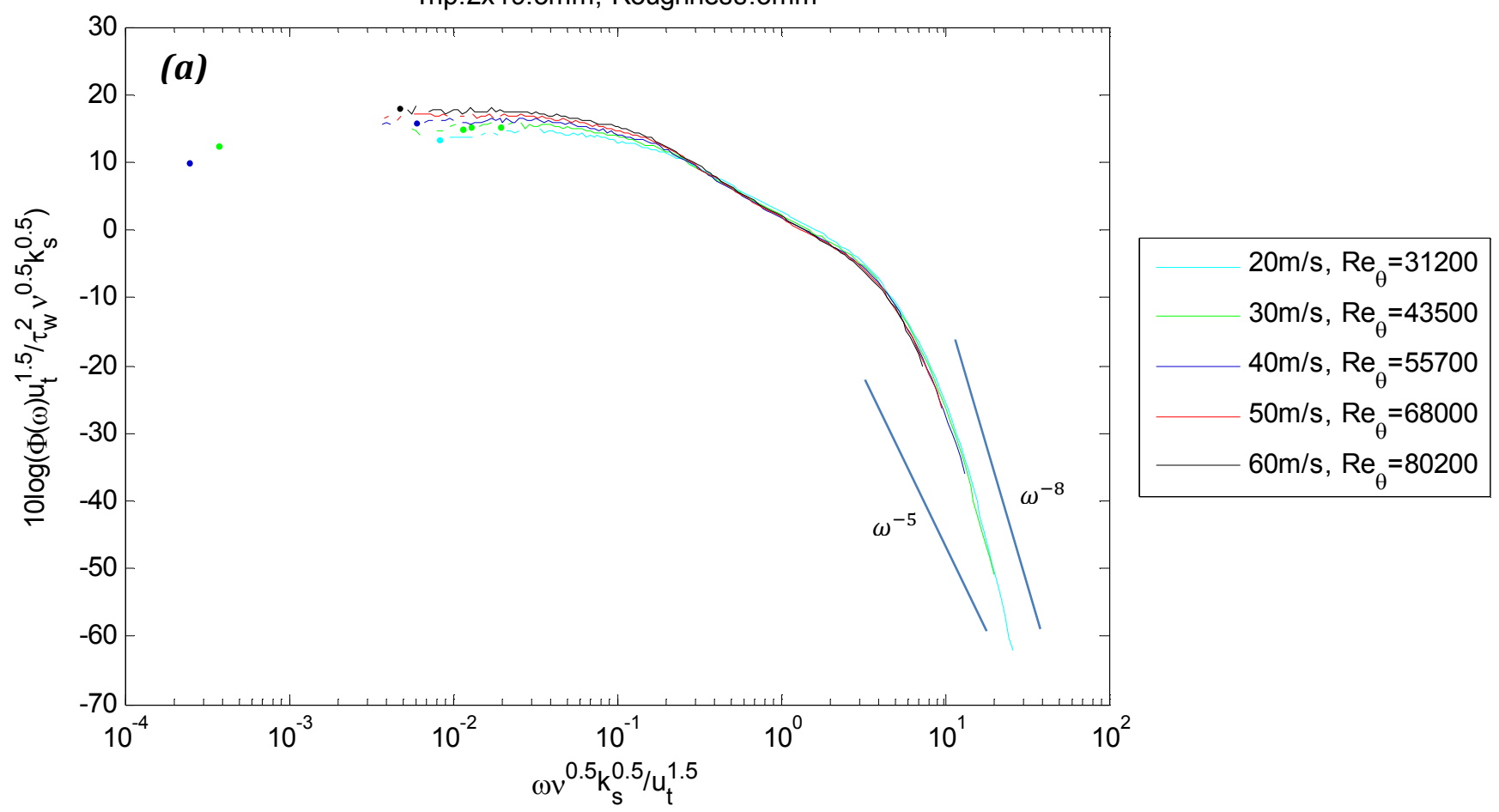

Proposed Roughness Based Inner Scaling

Trip:2x19.5mm, Roughness:3mm

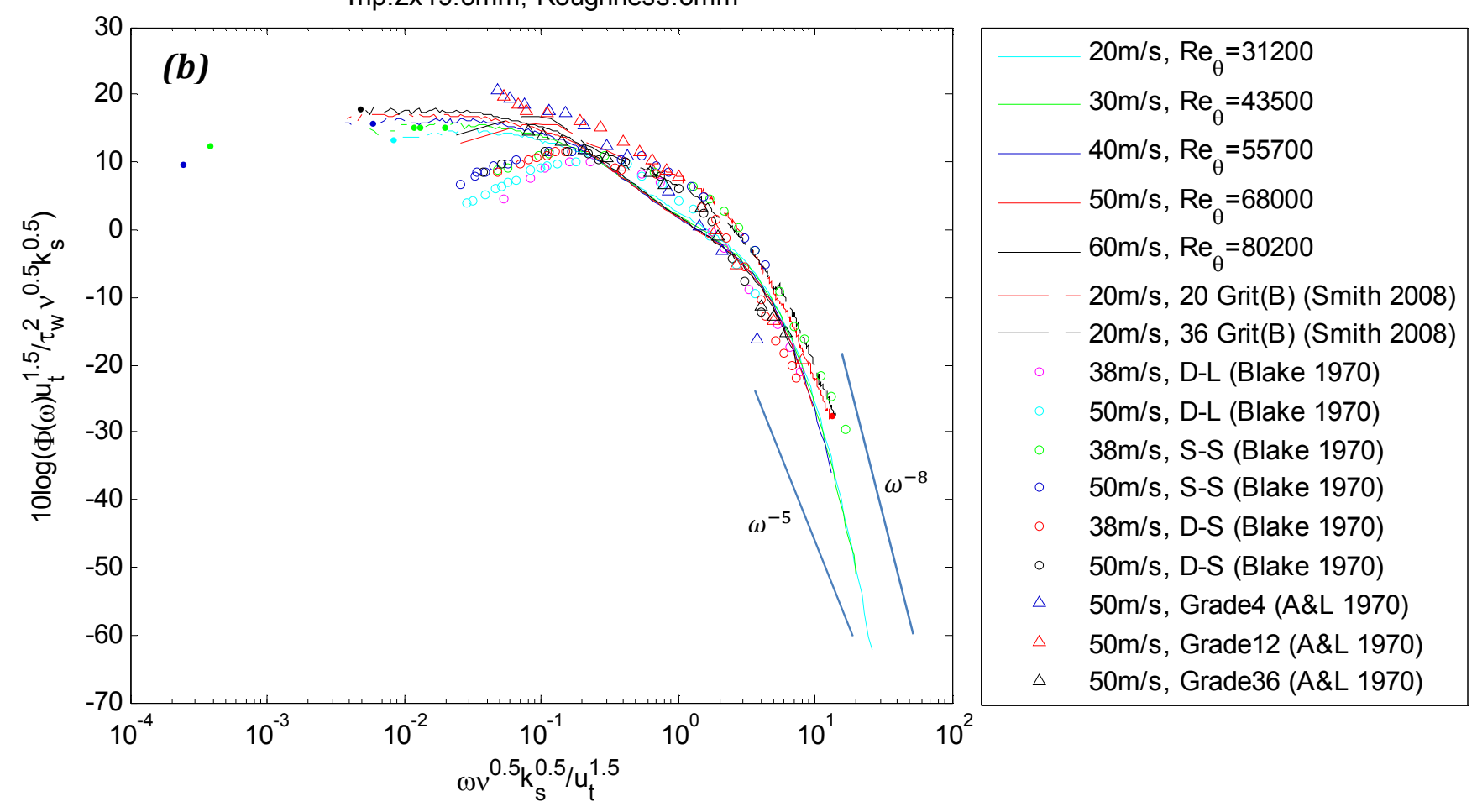

Figure 3.18. Wall pressure spectra for rough walls normalized on proposed roughness based inner variable scaling for (a) the present study only and (b) multiple studies for comparison. 


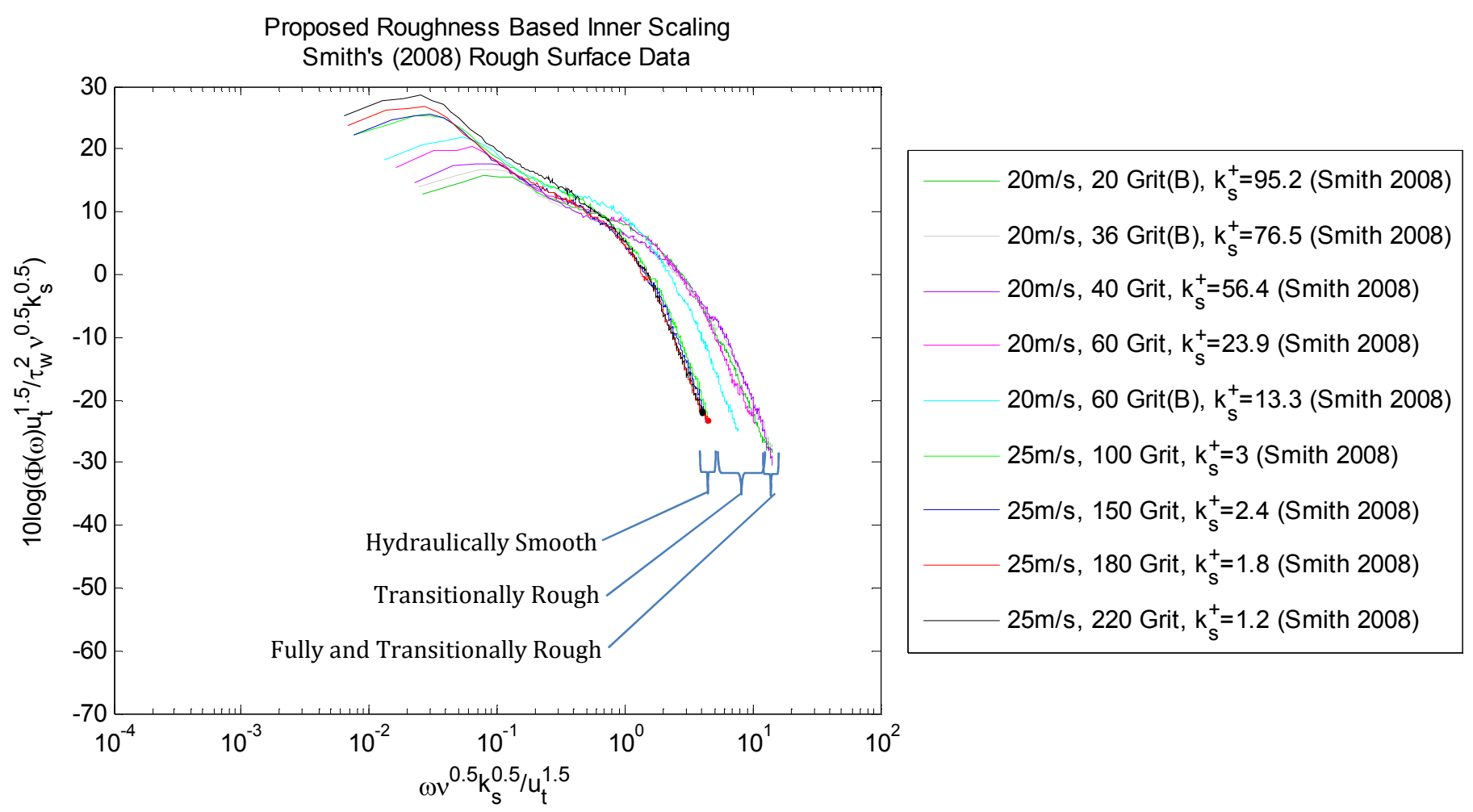

Figure 3.19. Wall pressure spectra from Smith (2008) for a range of roughness flows normalized on proposed roughness based inner variable scaling.

Figure 3.19 shows a wide range of flow roughness configurations taken from Smith (2008) and scaled on the proposed roughness based scaling. Fully rough, transitionally rough and hydraulically smooth flows are represented in this figure. While this scaling does not appear to collapse rough surfaces from the entire range of flow regimes, it is interesting to note that all the flow regimes do collapse between a finite band defined at the upper limit by fully rough flows, and at the lower limit by hydraulically smooth flows. All fully rough flows collapse together, while all hydraulically smooth flows collapse together along a separate line. Transitionally rough flows appear in between these two limits, with a clear roughness Reynolds number dependence influencing their positions. A possible physical explanation for this behavior stems from the inner boundary layer mean flow. From Schlichting (1979), the hydraulically smooth, transitional and fully rough flow regimes are largely defined based on the surface roughness's interference with the laminar sublayer. Specifically, the laminar sublayer is completely destroyed in fully rough flows, and remains largely unaffected for hydraulically smooth flows. Transitional flows see only partial destruction of the laminar sublayer. If it were assumed that the mean flow had some contribution to the turbulent structure generation and convection over rough surfaces very near the wall, then this would imply the presence of a partial or complete laminar sublayer would influence the high frequency wall pressure spectral behavior. As such, the complete lack of a laminar sublayer would prevent the fully rough high frequency spectra from collapsing on another frequency spectra that was influenced by the presence of a laminar sublayer. As what was found in Figure 3.19, this interaction would cause pressure spectra measured under transitionally rough flows with partially intact laminar sublayers to scale somewhere between hydraulically smooth and fully rough flows. 
In Figure 3.20, the rough wall pressure spectra are scaled using an alternate roughness based inner variable scaling. This scaling is based off of the proposed roughness based scaling, though using $k_{g}$ instead of $k_{s}$. This results in the scaling parameters $\Phi(\omega) u_{t}^{1.5} /\left(\tau_{w}^{2} v^{0.5} k_{g}^{0.5}\right) v s . \omega v^{0.5} k_{g}^{0.5} / u_{t}^{1.5}$. An improved collapse is again observed when compared to Blake's (1970) scaling, though this collapse is somewhat looser than what was found when using the other proposed scaling method implementing $k_{s}$. Specifically, almost all of Aupperle and Lambert's (1970) pressure spectra exhibit a roll off at much lower frequencies than a complete collapse would require. It is somewhat unclear on whether this deviation is specific to that study, though the variation is enough to question whether this scaling is more appropriate than the proposed scaling using $k_{s}$. The current set of results thus suggest that the use of $k_{s}$ over $k_{g}$ would be more appropriate, though further experimental investigations could show Aupperle and Lambert's data to be an anomaly. As the present conclusion is that the equivalent sand grain roughness height $k_{s}$ is more appropriate to use than the roughness height $k_{g}$, it would suggest that the entire roughness configuration, not just the geometric height, plays an important role in the production of the turbulent structures.

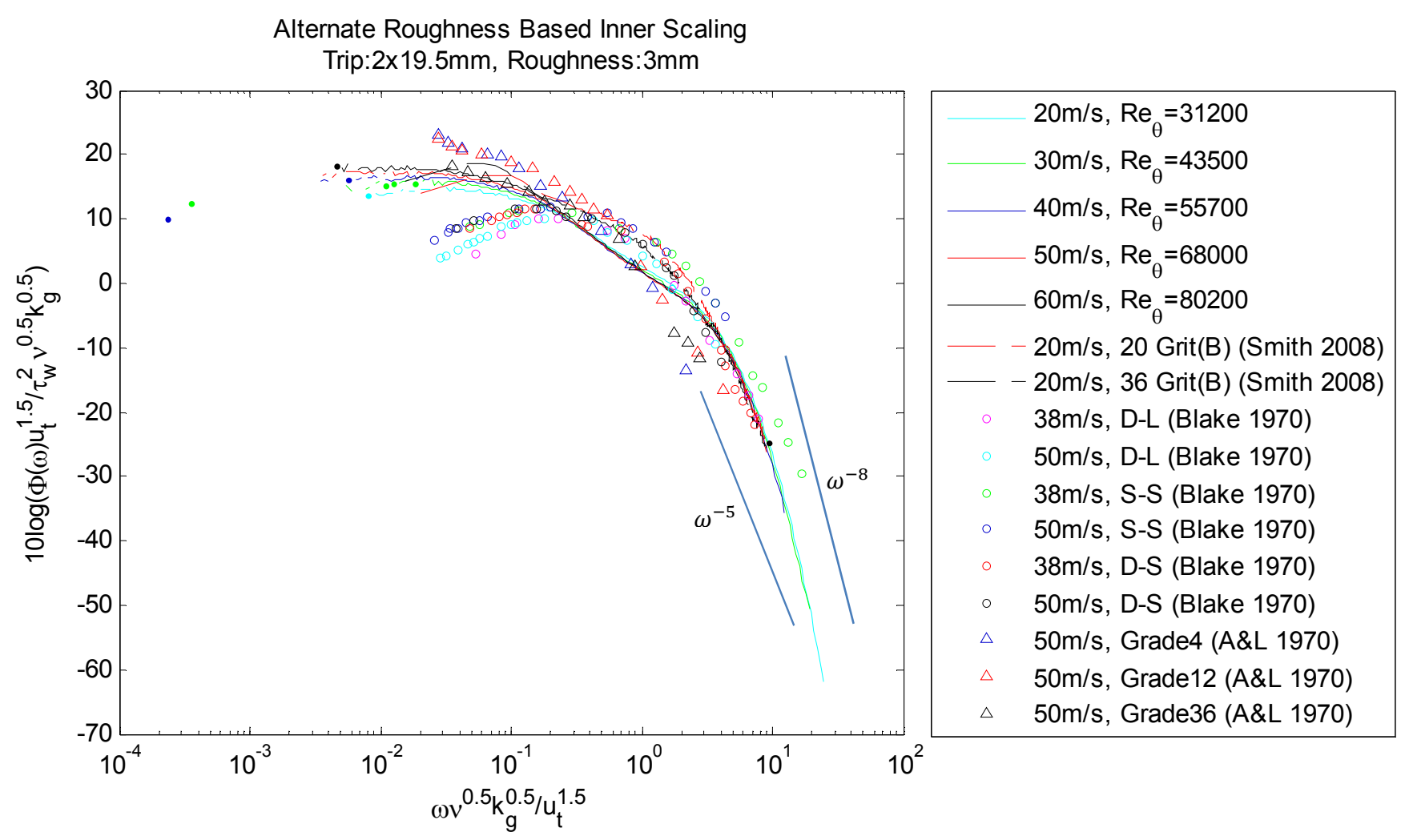

Figure 3.20. Wall pressure spectra for rough walls normalized on alternate roughness based inner variable scaling using $k_{g}$ instead of $k_{s}$.

As was seen for smooth wall flows, outer boundary layer variables are typically used to collapse the pressure spectra from low to mid spectral frequencies. If roughness height is kept to within a relatively small portion of the boundary layer thickness $\left(\delta / k_{g} \gtrsim 40\right)$ the effects of the roughness elements are not expected to extend far into the logarithmic region of the boundary layer such that similarity laws can be expected (Jiménez 2004). Thus for 
rough wall flows meeting these criteria, the same classical and mixed outer variable scales $\Phi(\omega) u_{t} /\left(\tau_{w}^{2} \delta\right) v s . \omega \delta / u_{t}$ and $\Phi(\omega) U_{e} /\left(\tau_{w}^{2} \delta^{*}\right) v s . \omega \delta^{*} / U_{e}$ used for smooth wall spectra should also collapse the rough wall spectra along the low to mid frequency regions.

The rough wall pressure spectra are shown in Figure 3.21 normalized on classical and mixed outer variable scaling along with the rough wall data from Blake (1970). Other previously examined rough wall studies are not shown normalized using these outer variable scales due to either a lack of reported variables required for these normalizations or due to their incompatible outer boundary layer regions. For the present data, an excellent collapse is shown along the low and mid frequency regions as was expected. Blake's data is also shown to collapse at comparable spectral levels in these regions, though the mixed variable scaling appears to form a much tighter collapse between the data sets than what is shown using classical scaling. While this could be an effect of the surface roughness configuration altering the boundary layer shape factor, the effects on the outer region boundary layer turbulence should theoretically be scalable on $u_{t}$ alone as long as the roughness height is kept relatively small to the boundary layer thickness (Jiménez 2004). As such, this comparison makes it unclear on whether this discrepancy could be attributable to the different natures of the outer variable scales, or from experimental error differences found between the studies. Additionally, just before the spectral peak at $/ u_{t} \cong 60\left(\omega \delta^{*} / U_{e} \cong 0.4\right)$, Blake's data is shown to decrease more rapidly at low frequencies than what was found in the present study's data. However, with the present study seeming to follow the low trend exhibited by the other rough wall studies in the previous figures, it could be argued that Blake's data set may have over corrected for the low frequency background acoustics.

It is interesting to note that neither Blake's data, nor Aupperle and Lambert's (1970) data sets from Figure 3.18 to Figure 3.21 appear to show any clear overlap regions. Conversely, the data in the present study and that of Varano (2010) do exhibit clear mid frequency overlap regions. Varano, however, does not discuss these specific findings and cites that there may be some outer region interference present in his experiment due to possible floor and ceiling boundary layer interactions. Thus it is believed that the present study is the first to show a clearly defined pressure spectra overlap region for fully rough flows free of outer region interference. While there is some uncertainty about the amount of outer region interference present in Varano's pressure spectra, the overlap region found in the present study suggest that the rough wall pressure spectral overlap region is a real feature. Additionally, previous studies have shown an overlap region exists for transitionally rough flows and flows just after encountering surface roughness (Klewicki et al. 2008, Morrison 2007). The clearly defined overlap region found in the present study is thought to be a function of the high Reynolds numbers and scale ratios $\left(\delta / \mathrm{kg}_{g}\right)$ achieved when compared to previous studies that did not observe overlap regions over rough walls.

In Morrison (2007), the introduction of surface roughness is shown to shift a large amount of turbulent kinetic energy (TKE) towards the surface. This would equate to higher turbulence levels found along lower portions of the boundary layer, and should present itself as elevated spectral levels found along some of the lower frequencies. There is some evidence of this shown in Figure 3.21, where the low frequency peak is found to be much broader than what was found for the smooth wall spectra. It could therefore be argued that if the roll off region were to occur at the same normalized frequency as it would occur over the equivalent smooth wall spectra, the overlap region slope would have to be a function of 
the amount of TKE that was shifted towards the surface, altering the width of the low frequency spectral peak. This shift in TKE is most likely highly dependent on the roughness configuration, implying that the frequency dependence found in the overlap region would also have to be a function of the roughness configuration.

While further studies investigating various roughness configurations would need to be examined for verification of this theory suggesting an overlap region slope dependent on the surface roughness, some evidence of this is already present. For example, in the transitionally rough flow studied by Klewicki et al. (2008) where the TKE transport towards the surface would be minimal, a frequency dependence in the overlap region was reported as $\omega^{-1}$ and could be interpreted to be as high as $\omega^{-1.08}$. In the present study where a fully rough flow was observed with a larger associated TKE shift expected, a much steeper frequency dependence of $\omega^{-1.33}$ was found. In Varano (2010), while outer region interference may have influenced some of the overlap region results, the frequency dependence did vary by roughness configuration. Following the previous trend, the more sparsely spaced roughness configurations in Varano's study that were expected to have a lower TKE transport exhibited lower slopes in the overlap region when compared to those configurations expected to have a higher TKE transport.

In Bradshaw (1967), it was found that a significant fraction of the streamwise velocity fluctuation spectra correlated strongly with that of the surface pressure fluctuation spectra. It was found that a majority of the energy due to surface pressure fluctuations was produced in the same region as where the maximum turbulent intensities were located for the velocity spectra in the logarithmic region. Bradshaw was able to conclude in this paper that the pressure fluctuation spectrum was proportionally related to the velocity fluctuation spectrum in a boundary layer. One could thus relate the known roughness effect of retarding the boundary layer velocities as one nears the wall with the steeper frequency dependence found in the wall pressure spectrum overlap region.

In Figure 3.22, the rough wall pressure spectra is compared with the smooth wall pressure spectra normalized on classical and mixed outer variable scaling. The mixed outer variable scaling again appears to form a better collapse between the smooth and rough wall pressure spectra, especially along the frequency axis. Using the mixed outer variable scaling, the low frequency spectral peaks and roll off frequencies are shown to align for similar Reynolds number flows over both smooth and rough walls. Once the overlap region is reached, the steeper slope exhibited by the rough wall spectra is clearly evident, resulting in a roll off frequency beginning at lower relative pressure levels. From Bradshaw (1967), this would be expected as the streamwise velocity fluctuations would see some retardation due to the roughness elements. Also apparent in this figure is the wider peak exhibited by the rough wall pressure spectra. This would correspond to a shift in some of the turbulence towards the wall due to roughness. For comparison, the low frequency bound of the overlap region appears to start near $\omega \delta^{*} / U_{e} \cong 0.33$ over smooth walls, and is delayed until approximately $\omega \delta^{*} / U_{e} \cong 2.74$ over the currently configured rough wall flows. 

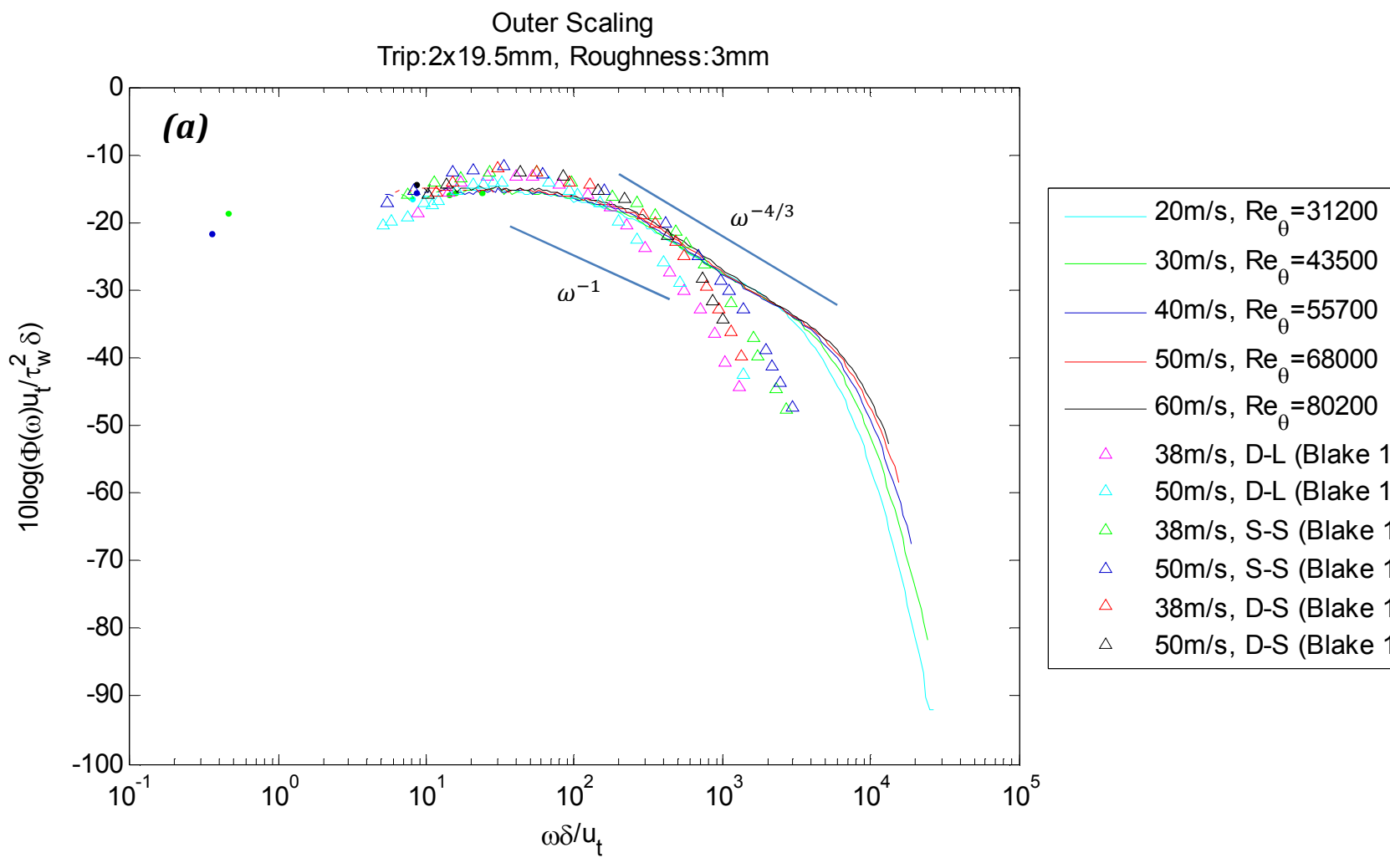

38m/s, D-L (Blake 1970)

$50 \mathrm{~m} / \mathrm{s}, \mathrm{D}-\mathrm{L}$ (Blake 1970)

$38 \mathrm{~m} / \mathrm{s}$, S-S (Blake 1970)

$50 \mathrm{~m} / \mathrm{s}$, S-S (Blake 1970)

$38 \mathrm{~m} / \mathrm{s}$, D-S (Blake 1970)

$\triangle \quad 50 \mathrm{~m} / \mathrm{s}$, D-S (Blake 1970)

Outer Scaling

Trip:2x19.5mm, Roughness:3mm

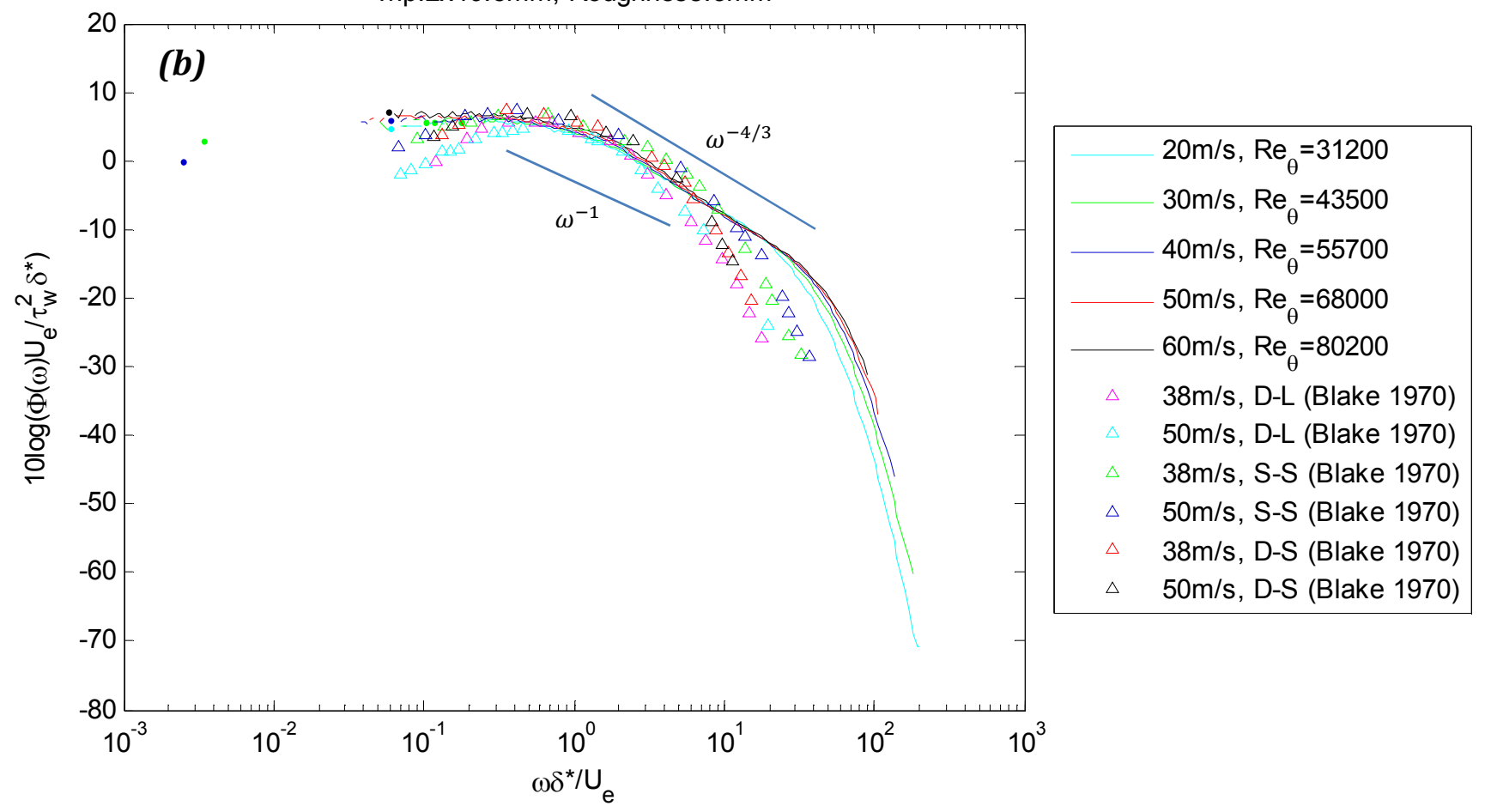

Figure 3.21. Wall pressure spectra for rough walls normalized on (a) classical outer variable scaling and (b) mixed outer variable scaling. 


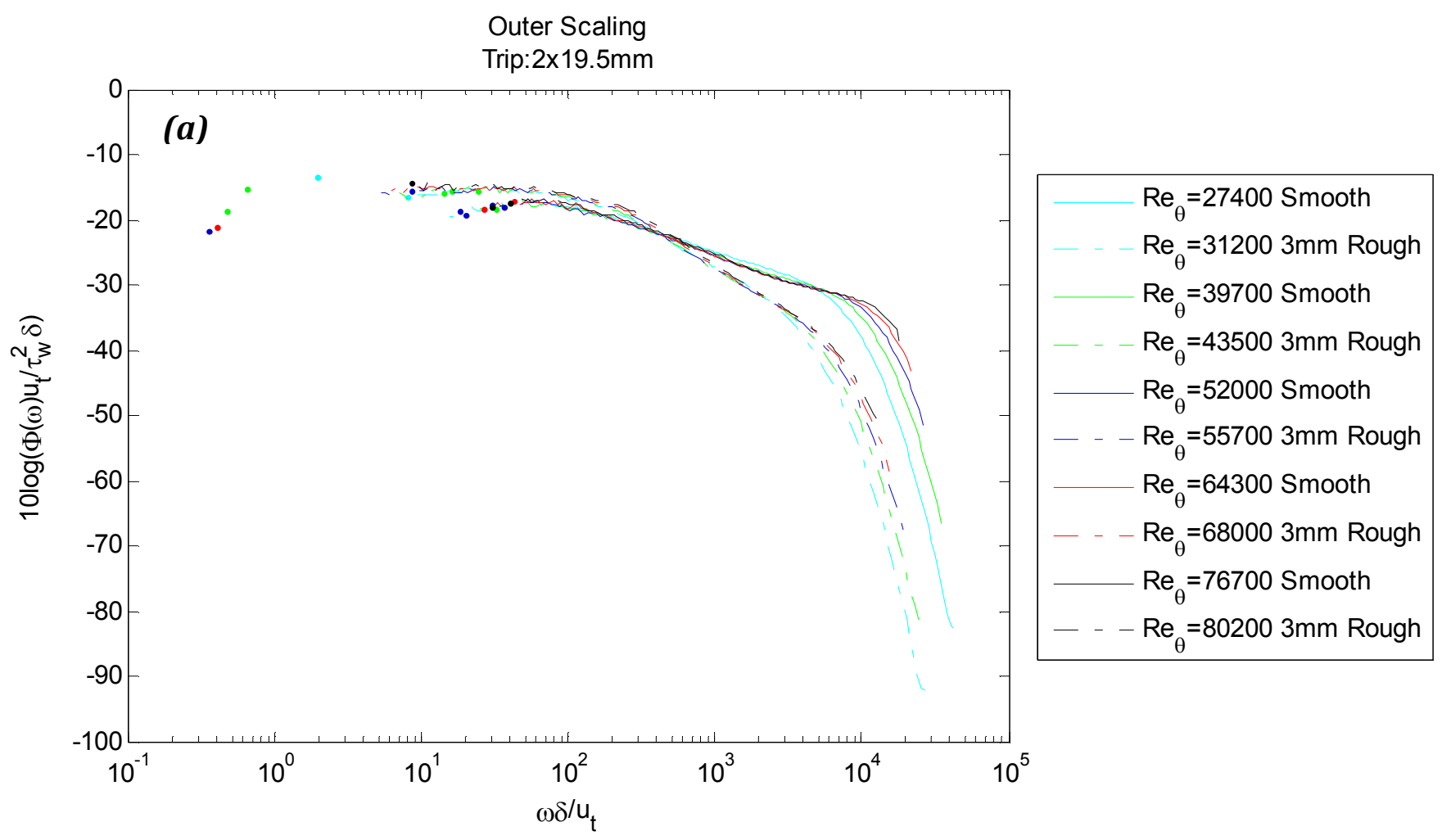

Outer Scaling

Trip:2x19.5mm

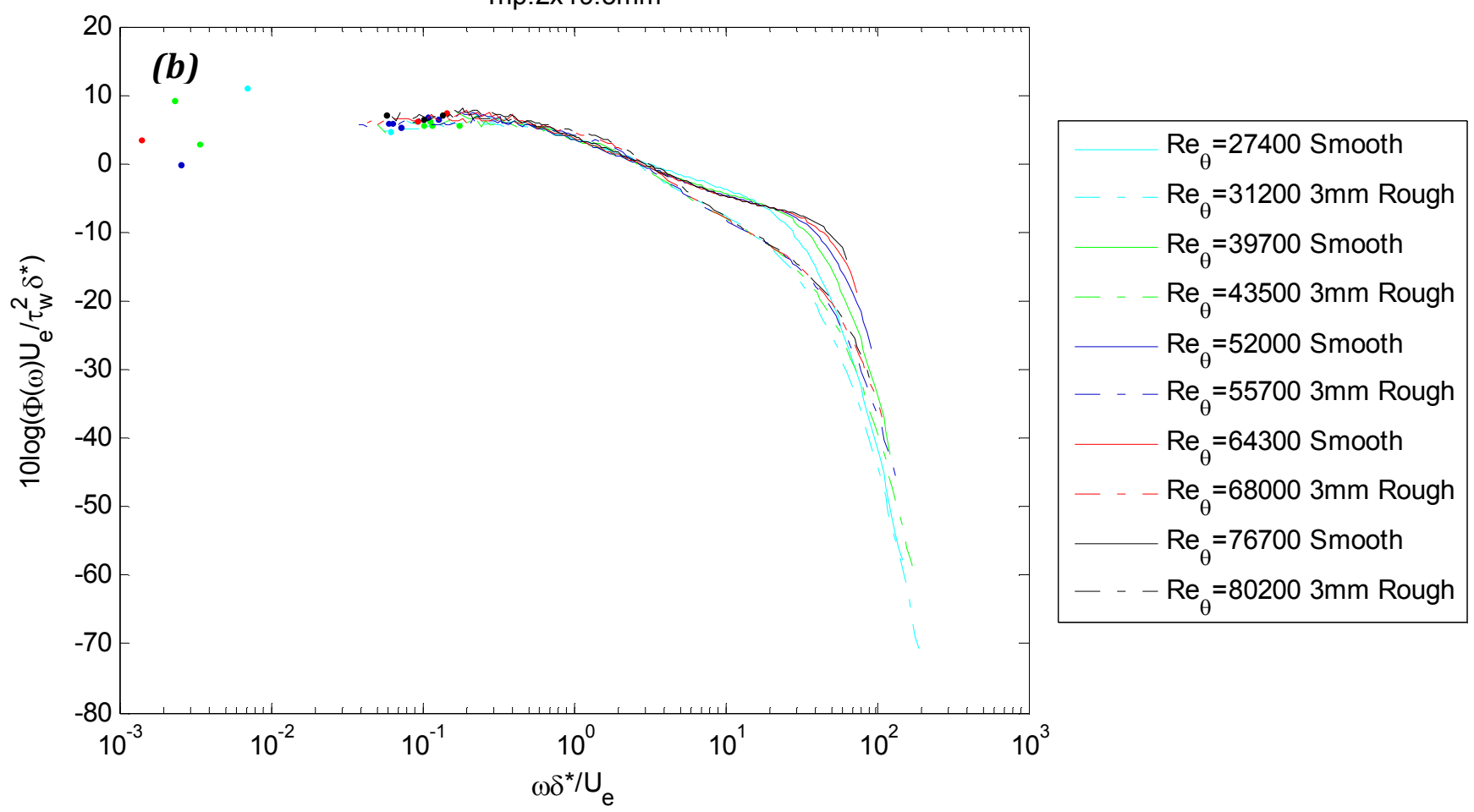

Figure 3.22. Wall pressure spectra for smooth and rough walls overlaid, normalized (a) classical outer variable scaling and (b) mixed outer variable scaling. 


\subsubsection{Coherence}

Wall surface pressure spectra coherence was found using Equation 3.17, where $\Gamma$ is coherence and $\phi_{p_{i} p_{j}}$ is the wall pressure cross-spectrum between microphones locations $i$ and $j$.

$$
\Gamma^{2}=\frac{\left|\phi_{p_{i} p_{j}}^{2}\right|}{\left|\phi_{p_{i} p_{i}}\right|\left|\phi_{p_{j} p_{j}}\right|}
$$

The wall pressure spectra coherence over streamwise separations $\xi$ is mapped for smooth wall flows between 22.4 and $67.2-\mathrm{m} / \mathrm{s}$ in Figure 3.23, and for rough wall flows between 20 and $60-\mathrm{m} / \mathrm{s}$ in Figure 3.24. The frequency $\omega$ is normalized on boundary layer displacement thickness $\delta^{*}$ and edge velocity $U_{e}$, while streamwise separation distances $\xi$ are normalized on boundary layer displacement thickness $\delta^{*}$. The tick-marks on the right hand side of each plot represent the actual microphone separation locations, with data between these locations being interpolated.

The smooth wall pressure spectral coherence in Figure 3.23 is shown to maintain values of $\Gamma^{2}>0.2$ within a streamwise separation of $10 \delta^{*}$ for frequencies below $\omega \delta^{*} / U_{e}=$ 0.73 . At frequencies below $\omega \delta^{*} / U_{e}=0.19$, the pressure spectra is shown to have larger coherence values of $\Gamma^{2}>0.4$ for separations of at least $28 \delta^{*}$. The larger coherence values found at lower frequencies suggests that the larger turbulent eddies responsible for low frequency signals have a longer persistence over greater distances than the smaller, higher frequency eddies, similar to what was found by Bull (1967). When comparing the smooth wall pressure spectra coherence results with the rough wall pressure spectra coherence maps seen in Figure 3.24, one sees the coherence values of $\Gamma^{2}>0.2$ at $\omega \delta^{*} / U_{e}=0.73$ only exist out to $4.1 \delta^{*}$. Additionally, for frequencies below $\omega \delta^{*} / U_{e}=0.19$, the pressure spectra coherence at large separations of at least $21 \delta^{*}$ rarely rises above $\Gamma^{2}>0.15$. The more rapid decrease in coherence of the wall pressure over large streamwise separations suggests an increase in the amount of interference and mixing caused by the roughness element protrusions into the boundary layer. 

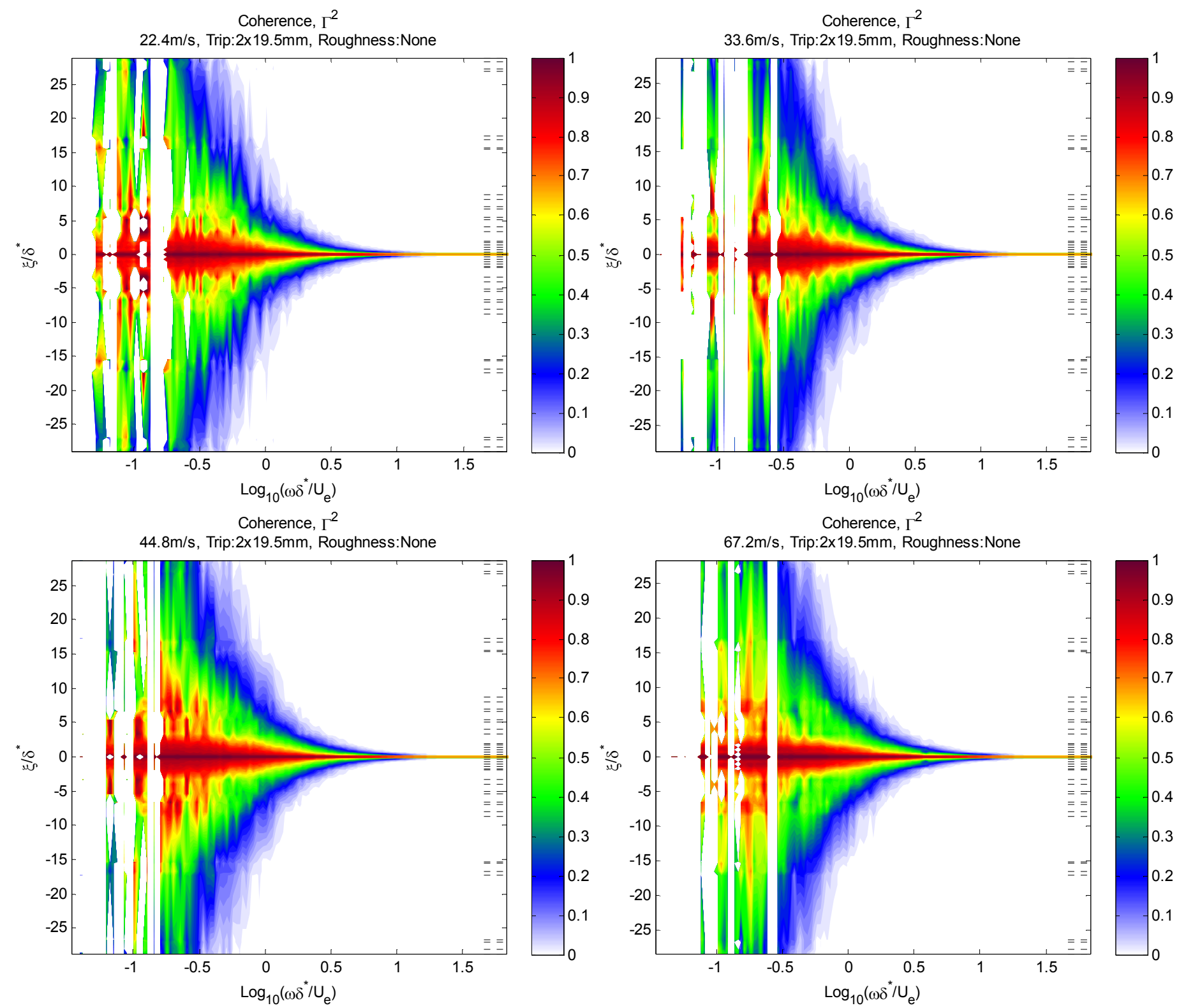

Figure 3.23. Smooth wall pressure spectra coherence for streamwise separations. 

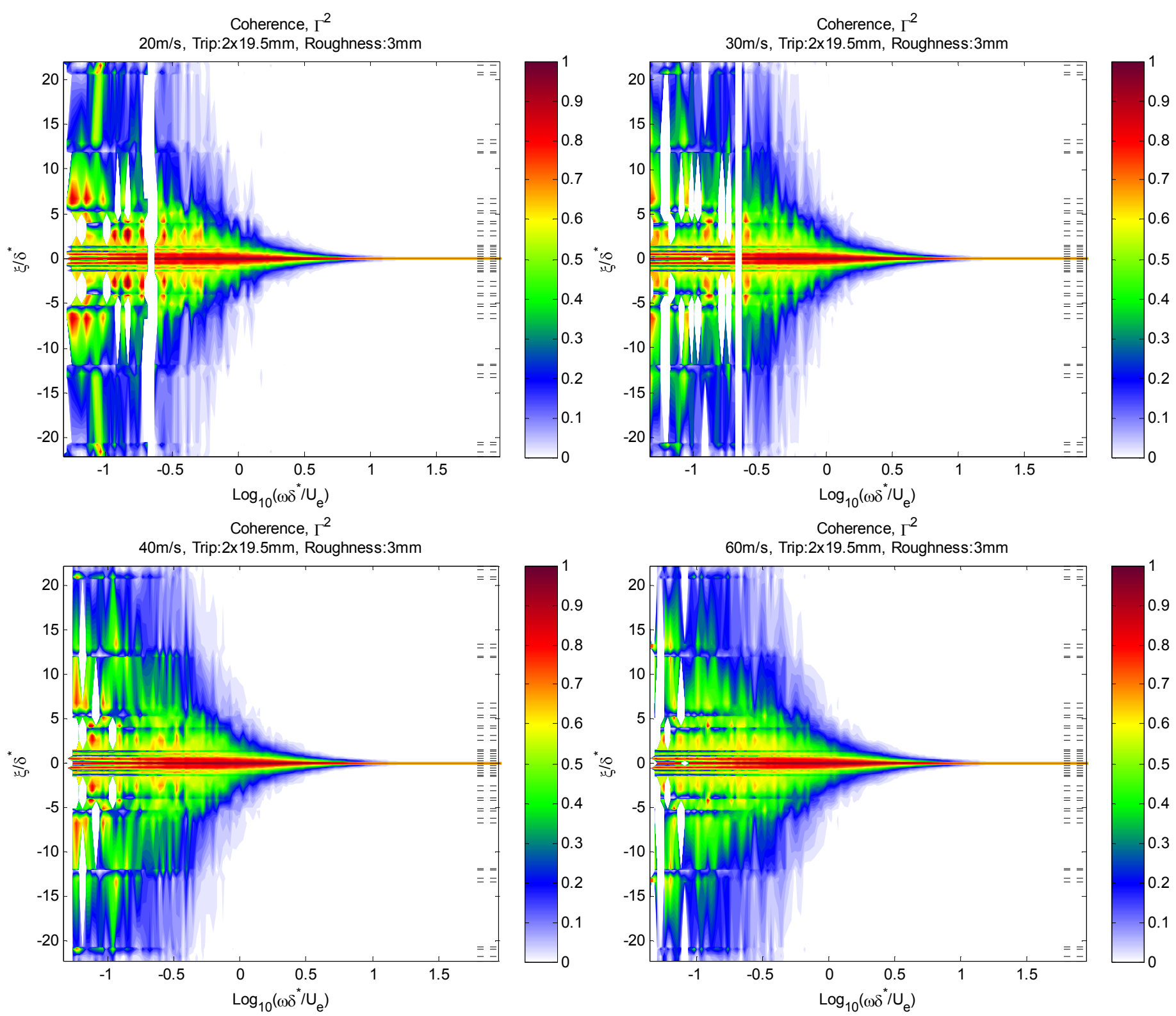

Figure 3.24. Rough wall pressure spectra coherence for streamwise separations.

The smooth and rough wall pressure spectral coherence over spanwise separations for speeds between 22.4 to $67.2-\mathrm{m} / \mathrm{s}$ and 20 to $60-\mathrm{m} / \mathrm{s}$ respectively are shown in Figure 3.25 and Figure 3.26. The expected rapid decrease in coherence due to increasing spanwise separation distances $\eta$ is evident in both smooth and rough wall cases as neither shows any significant coherence values beyond $4 \delta^{*}$. Coherence values at lower frequencies are shown to exhibit larger coherence values at comparable separations to those at higher frequencies as was seen in the streamwise case, though the lack of a freestream flow in the spanwise direction prevents these turbulent structures from being convected over any significant spanwise distances. 


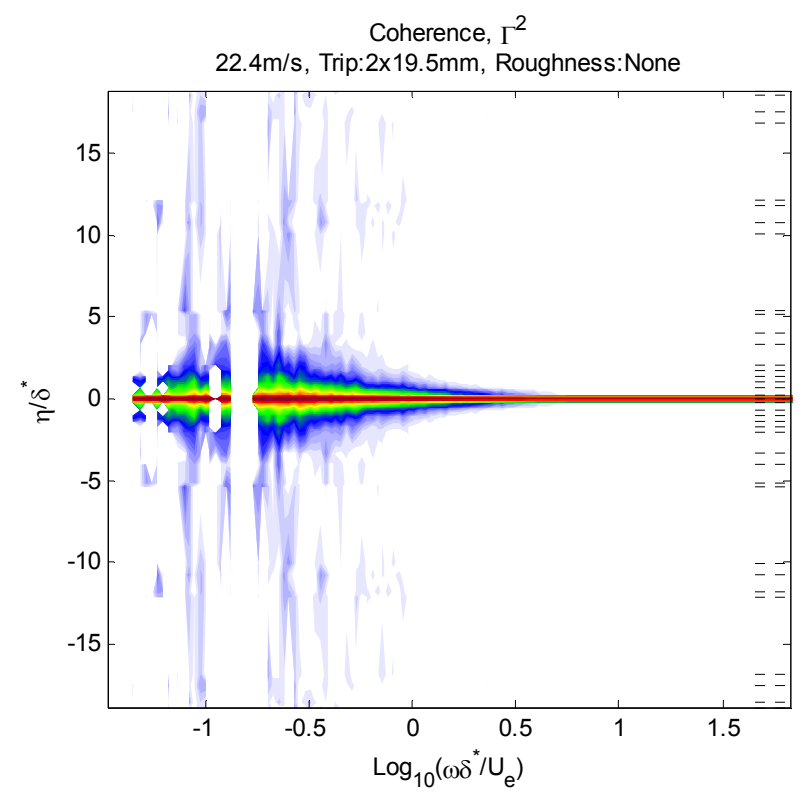

Coherence, $\Gamma^{2}$

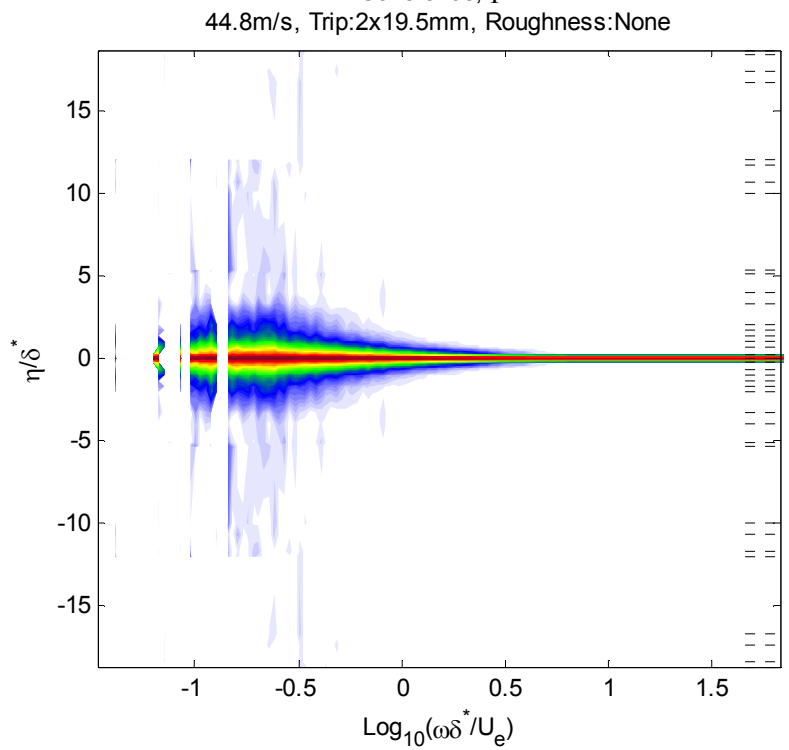

Coherence, $\Gamma^{2}$

$33.6 \mathrm{~m} / \mathrm{s}$, Trip:2x19.5mm, Roughness:None
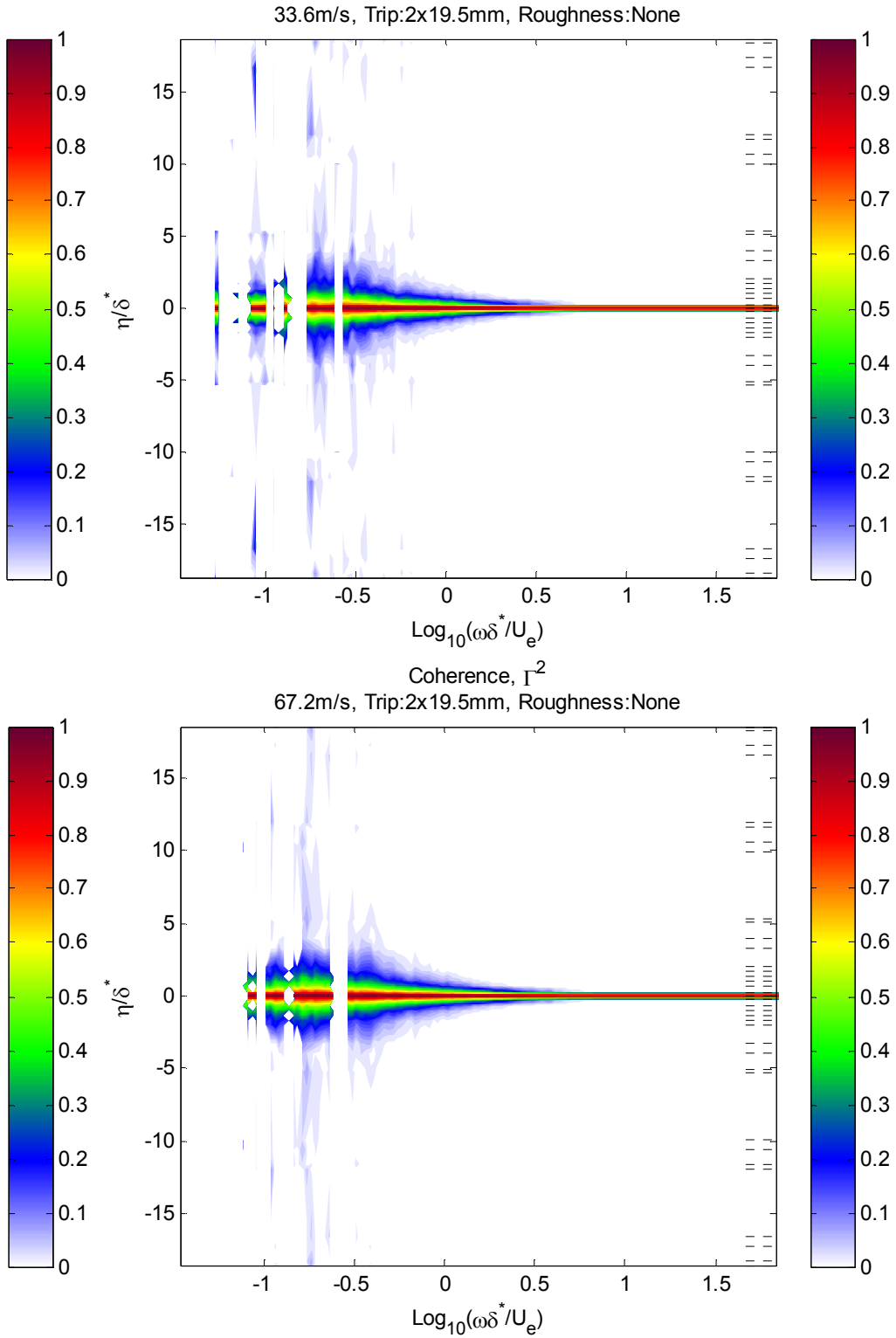

$67.2 \mathrm{~m} / \mathrm{s}$, Trip:2x19.5mm, Roughness:None

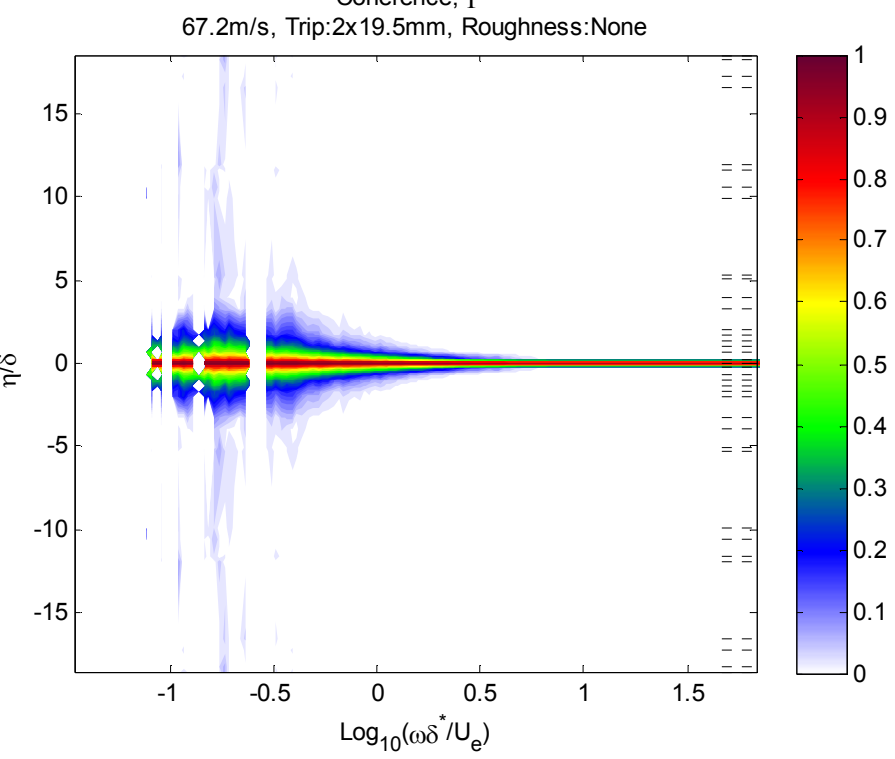

Figure 3.25. Smooth wall pressure spectra coherence for spanwise separations. 

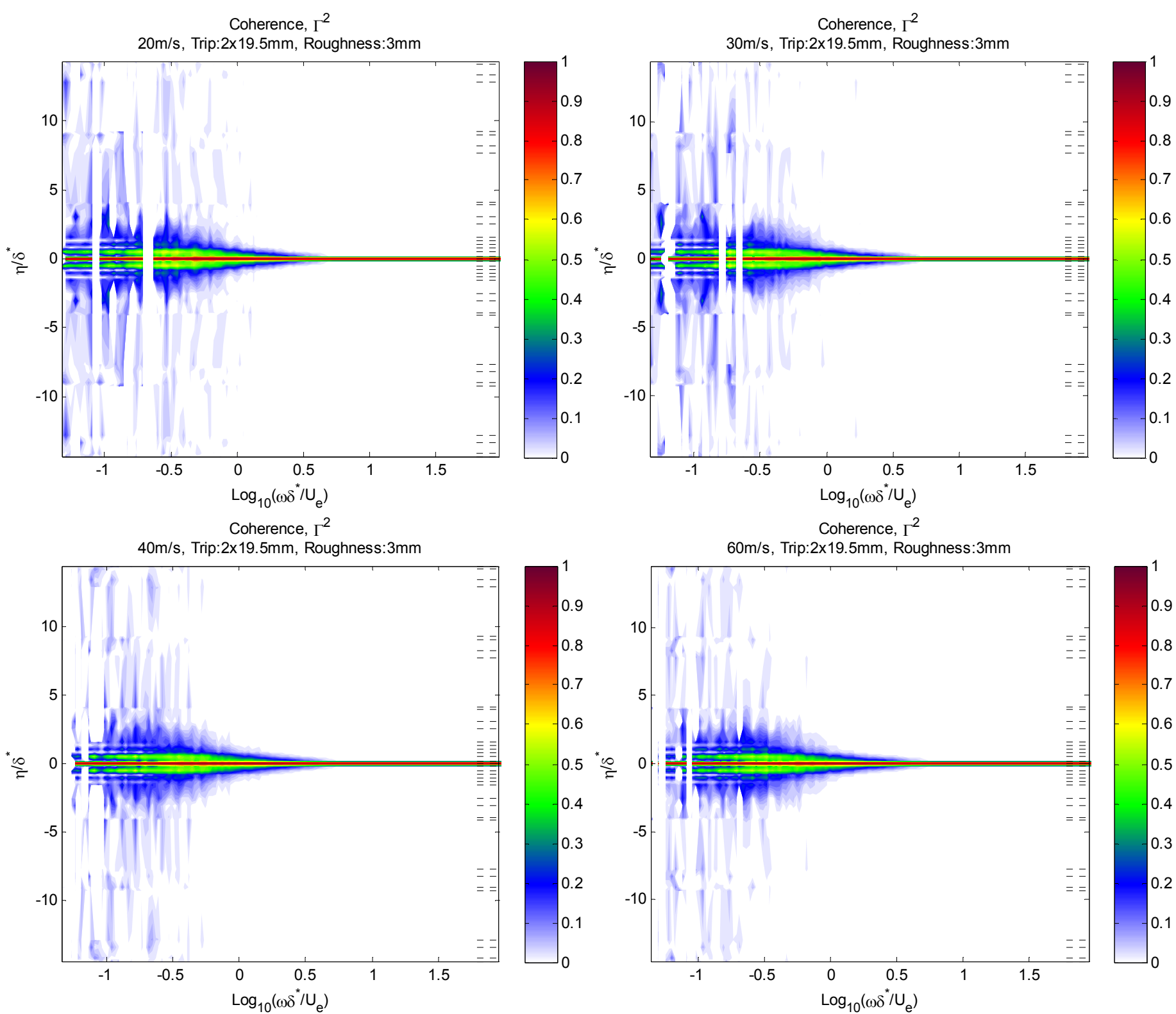

Figure 3.26. Rough wall pressure spectra coherence for spanwise separations.

The wall pressure spectra coherence decay over streamwise separations can be observed in Figure 3.27 and Figure 3.28 for a range of fixed frequencies. Here the separation distances are normalized by the fixed coherence frequency $\omega_{0}$ and the phase convection velocity $U_{c p}$. While a complete collapse is not fully observed, the upper limit of the coherence for the frequencies between the range of separations measured do appear to approach a single limit. As was found and described in Bull (1967) and Farabee and Casarella (1991), this would indicate that the turbulent structures decay based on the ratio of the wavelength the turbulence has been convected. This collapse, however, was shown to break down at low frequencies $\left(\omega \delta / u_{t}<50\right)$ by previous studies including Farabee and Casarella. Those frequencies that did collapse, however, were shown to follow the coherence decay function proposed by Corcos (1963). This coherence decay function is 
shown in Equation 3.18, where the decay rate is based on a constant $C_{1}$ and the phase angle $\theta(\omega, \xi)=\omega \xi / U_{c p}$.

$$
\Gamma(\omega, \xi)=e^{-C_{1}\left|\omega \xi / U_{c p}\right|}
$$

Equation 3.18

Following Farabee and Casarella, the constant $C_{1}$ is typically found to be between 0.10 and 0.19 for smooth walls, though it has been reported to be dependent on the freestream flow velocity. When this relation was fit to the present data as shown in Figure 3.27 and Figure 3.28, Reynolds number dependence was also observed. The calculated decay constant for each flow configuration is shown in Table 3.4. Interestingly, the coherence function still appears to be applicable to rough wall flows, though the decay constant increases significantly over rough walls owing to the more rapid coherence decay rate. As both smooth and rough wall flows appear to obey the same coherence decay function, it can be concluded that the presence of roughness does not significantly disrupt the pressure decay mechanism other than drastically increasing its rate. Additionally, since both surface conditions exhibit a moderate collapse in these figures, it could be concluded that the pressure spectra do in fact decay approximately the same over equal proportions of the wavelength fractions convected for rough surfaces as well as smooth.

Table 3.4. Calculation of coherence function constant $C_{1}$.

\begin{tabular}{ccccccccc}
$\begin{array}{c}\text { Speed } \\
(\mathbf{m} / \mathbf{s})\end{array}$ & $\mathbf{R e}_{\odot}$ & $\mathbf{C}_{\mathbf{1}}$ & Configuration & & $\begin{array}{c}\text { Speed } \\
(\mathbf{m} / \mathbf{s})\end{array}$ & $\mathbf{R e}_{\odot}$ & $\mathbf{C}_{\mathbf{1}}$ & Configuration \\
\hline 22.4 & 27400 & 0.145 & Smooth & & 20 & 31200 & 0.185 & 3-mm Rough \\
33.6 & 39700 & 0.130 & Smooth & & 30 & 43500 & 0.180 & 3-mm Rough \\
44.8 & 52000 & 0.120 & Smooth & & 40 & 55700 & 0.180 & 3-mm Rough \\
56 & 64300 & 0.115 & Smooth & & 50 & 68000 & 0.170 & 3-mm Rough \\
67.2 & 76700 & 0.105 & Smooth & & 60 & 80200 & 0.165 & 3-mm Rough
\end{tabular}


Coherence, $\Gamma$, by Frequency

22.4m/s, Trip:2x19.5mm, Roughness:None

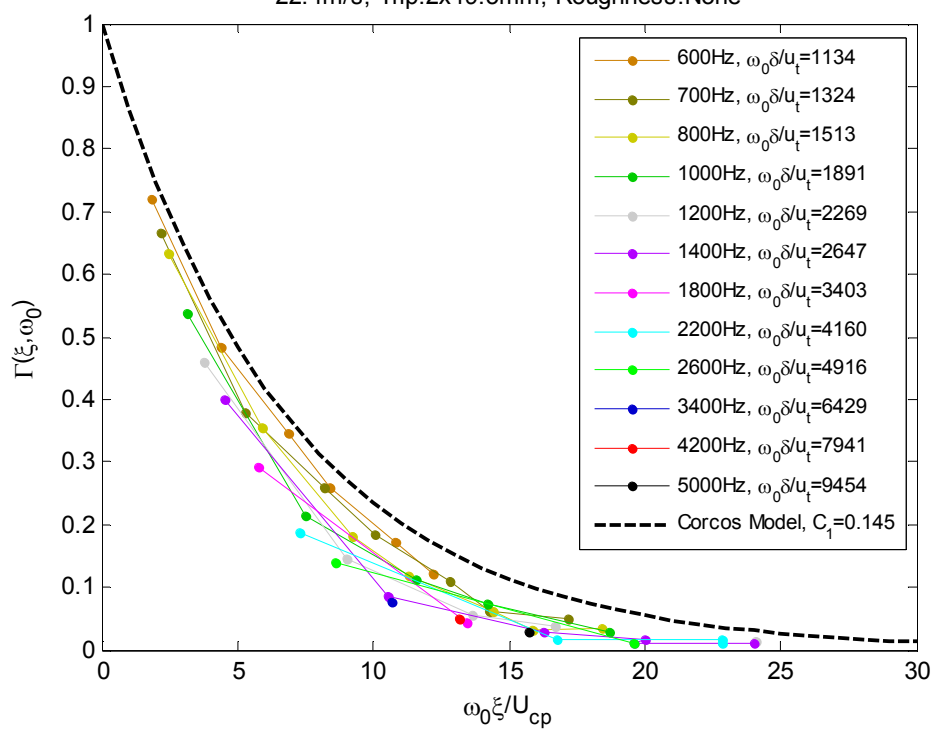

Coherence, $\Gamma$, by Frequency

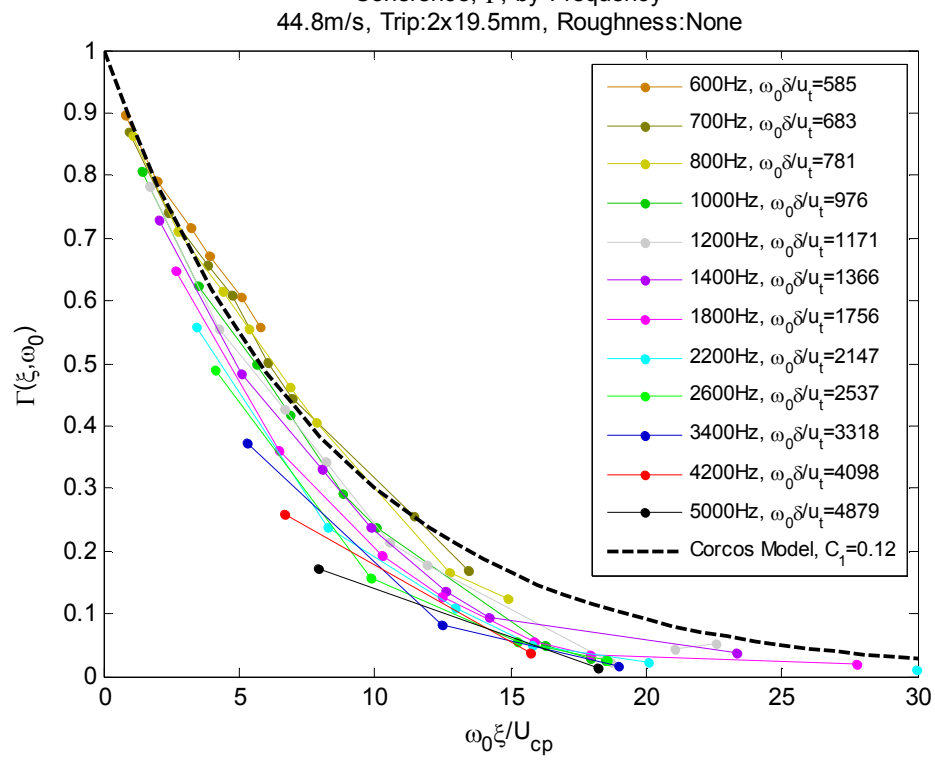

Coherence, $\Gamma$, by Frequency

$33.6 \mathrm{~m} / \mathrm{s}$, Trip:2x19.5mm, Roughness:None

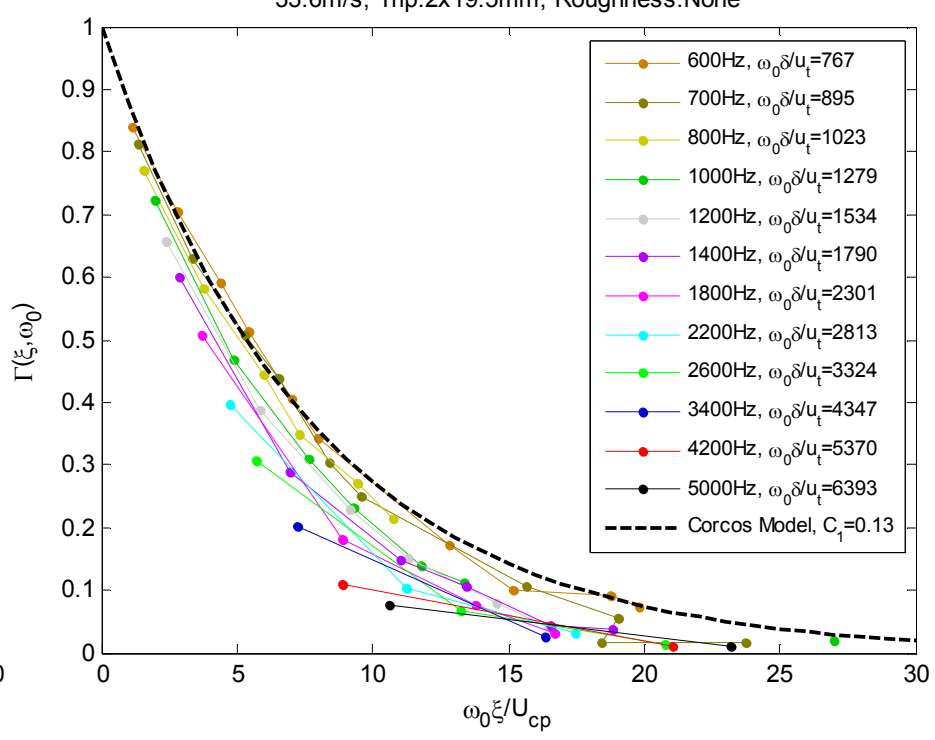

Coherence, $\Gamma$, by Frequency

$67.2 \mathrm{~m} / \mathrm{s}$, Trip:2x19.5mm, Roughness:None

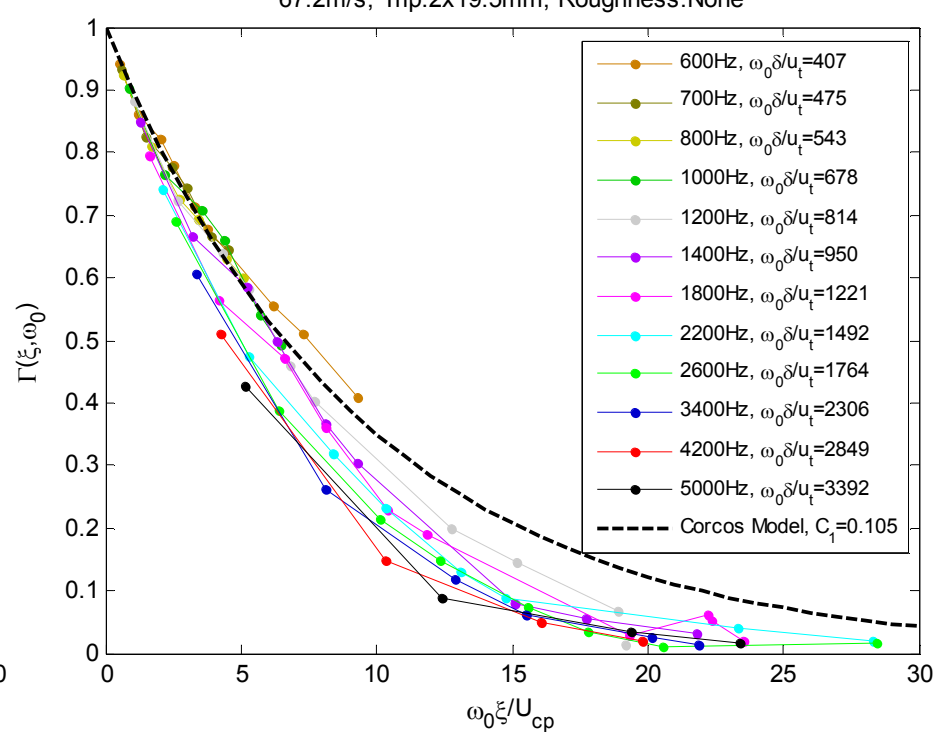

Figure 3.27. Smooth wall pressure spectra coherence for fixed frequencies by phase angle. 
Coherence, $\Gamma$, by Frequency

20m/s, Trip:2x19.5mm, Roughness:3mm

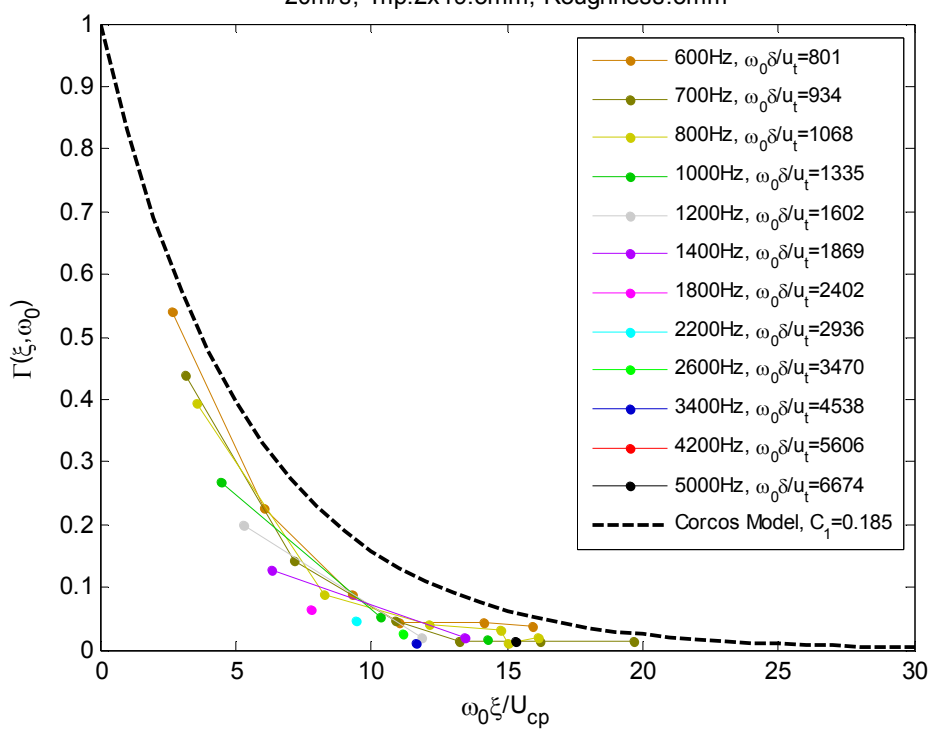

Coherence, $\Gamma$, by Frequency

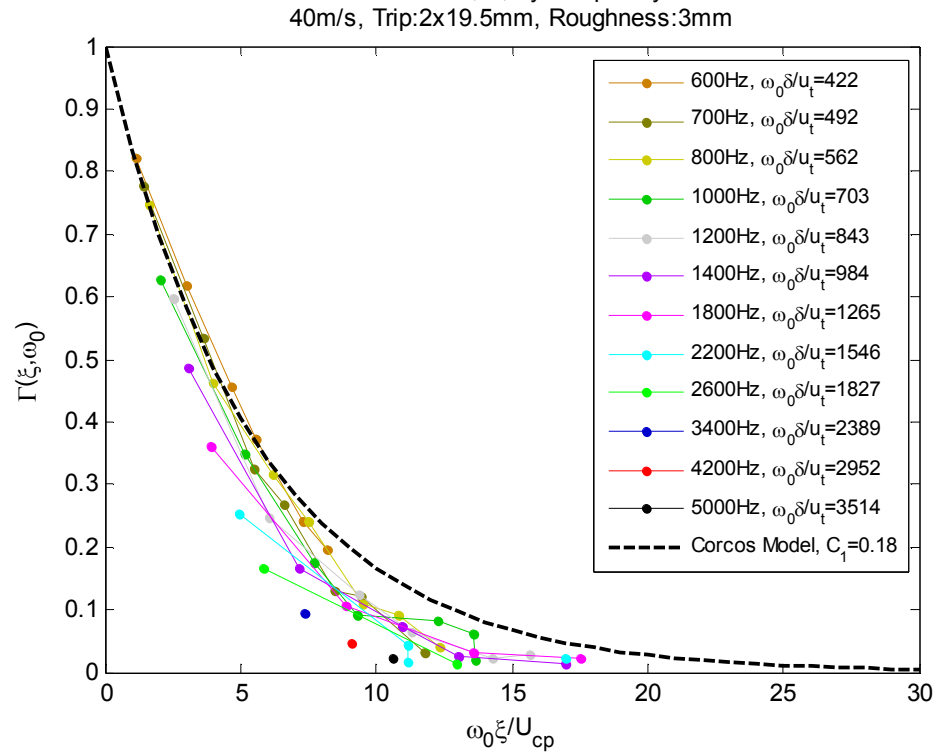

Coherence, $\Gamma$, by Frequency

$30 \mathrm{~m} / \mathrm{s}$, Trip: $2 \times 19.5 \mathrm{~mm}$, Roughness:3mm

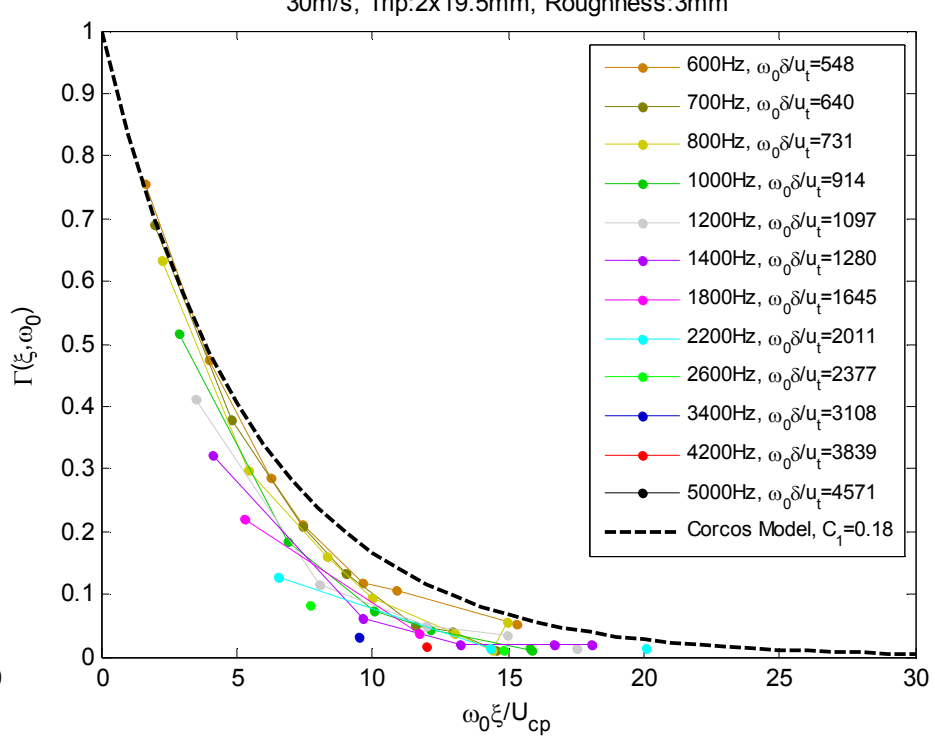

Coherence, $\Gamma$, by Frequency

$60 \mathrm{~m} / \mathrm{s}$, Trip:2x19.5mm, Roughness:3mm

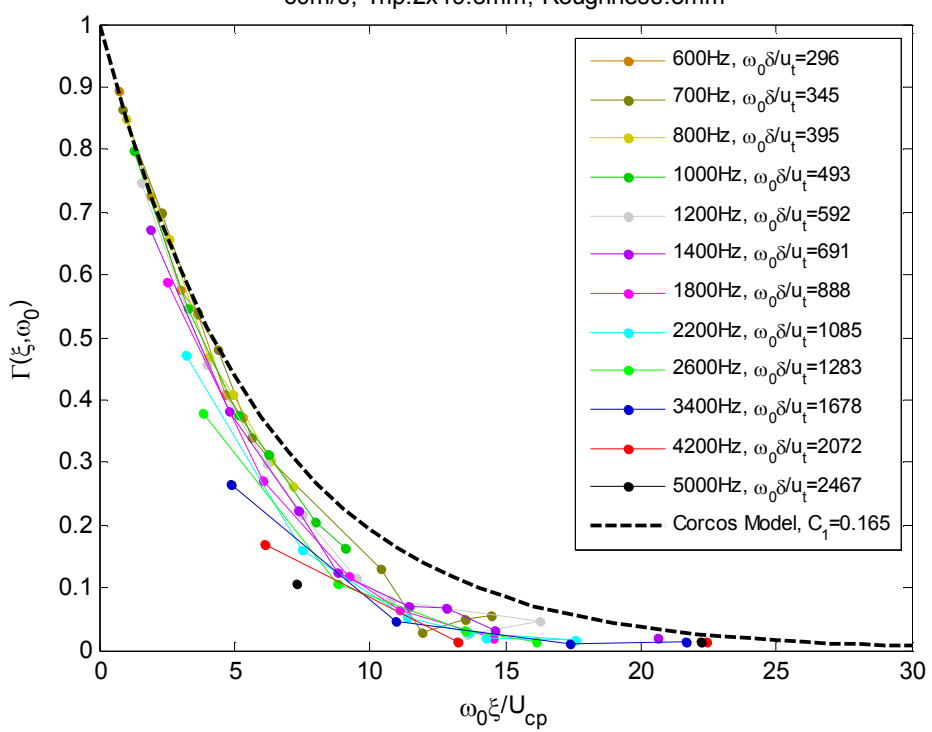

Figure 3.28. Rough wall pressure spectra coherence for fixed frequencies by phase angle.

\subsubsection{Correlation}

The wall pressure space-time correlation $R_{p p}$ is defined as the correlation between the pressure at a location $x$ and time $t$, and the pressure at location $x+s$ and time $t+\tau$ as seen in Equation 3.19. Due to the two-dimensionality and limited boundary layer growth near the microphone array, the boundary layer and associated turbulences were assumed to be homogeneous allowing $R_{p p}$ to be independent of $x$ and $t$.

$$
R_{p p}(s, \tau) \equiv\langle p(x, t) p(x+s, t+\tau)\rangle
$$

Figure 3.29 and Figure 3.30 show the space-time correlations of the wall surface pressure over smooth walls at 33.6 and $67.2-\mathrm{m} / \mathrm{s}$ and rough walls at 20 and $60-\mathrm{m} / \mathrm{s}$ for streamwise separations $\xi$ normalized on boundary layer displacement thickness $\delta^{*}$. The 
time delay $\tau$ has been normalized about edge velocity $U_{e}$ and boundary layer displacement thickness $\delta^{*}$. The tick-marks on the right hand side of each plot represent the actual microphone separations, where data between these locations has been interpolated.

The smooth surface correlation plots in Figure 3.29 show the normalized convective ridge to be nearly identical at both 33.6 and $67.2-\mathrm{m} / \mathrm{s}$. The convective ridge in this figure is shown to have a higher slope for larger $\xi / \delta^{*}$ separations when compared to smaller ones. This results from the smaller turbulent eddy structures dominating the pressure field over short distances as initially described in Bull (1967). Since it is known these smaller structures typically reside in the slower, inner portions of the boundary layer, they are convected at lower velocities than the larger turbulent structures that tend to dominate over larger distances. Thus as the smaller turbulent structures decorrelate, the turbulent structures located in the outer portions of the boundary layer become more prevalent, increasing the mean integrated boundary layer convective velocity. This in turn results in the larger convective ridge slopes seen in Figure 3.29 and Figure 3.30 as well as the increase in convection velocities displayed in Figure 3.43.

The plots in Figure 3.30 show the space-time correlation over rough walls. Here again, the slope of the convective ridge was found to be higher for larger separations as suggested by the rough wall convection velocity from Figure 3.43. When compared to the smooth surface results in Figure 3.29, the rough wall convective ridges are shown to have a much wider ridge, especially over smaller separation distances. While the turbulence structures located in the inner portions of the boundary layer are typically modeled as attached eddies over smooth walls, the wider convective ridge that was found indicates a much wider range of eddy convections can be found over rough walls. It is known that the pressure correlation is dominated by the small eddies near the surface over short distances, and that large eddies in the outer boundary layer regions begin to dominate over larger distances. Thus the trend of wider convective ridges only being found over short distances on rough walls suggests that only the inner boundary layer convection velocities are becoming mixed due to the presence of roughness. Outer region convection velocities may be affected by the surface roughness as will be discussed in section 3.4.6, though this appears to occur without any associated increase in convection velocity variation shown by the similar ridge width that is found over both rough and smooth walls for large separations.

Figure 3.31 and Figure 3.32 show the space-time correlation plots with additional markings for the measured maximum correlation locations corresponding to the convective ridges for smooth and rough walls at select conditions. Note that the measured convective ridge locations found by Bull (1967) and Blake (1970) for smooth walls and by Blake (1970) over two rough wall configurations have also been added to these figures for comparison. Figure 3.31 shows slight discrepancies between the smooth wall data sets, though only for larger separations, while the rough wall data sets in Figure 3.32 appear to show increased variation at smaller separations. The rough wall convective ridge variations are believed to be due to the surface roughness used in each data set. 


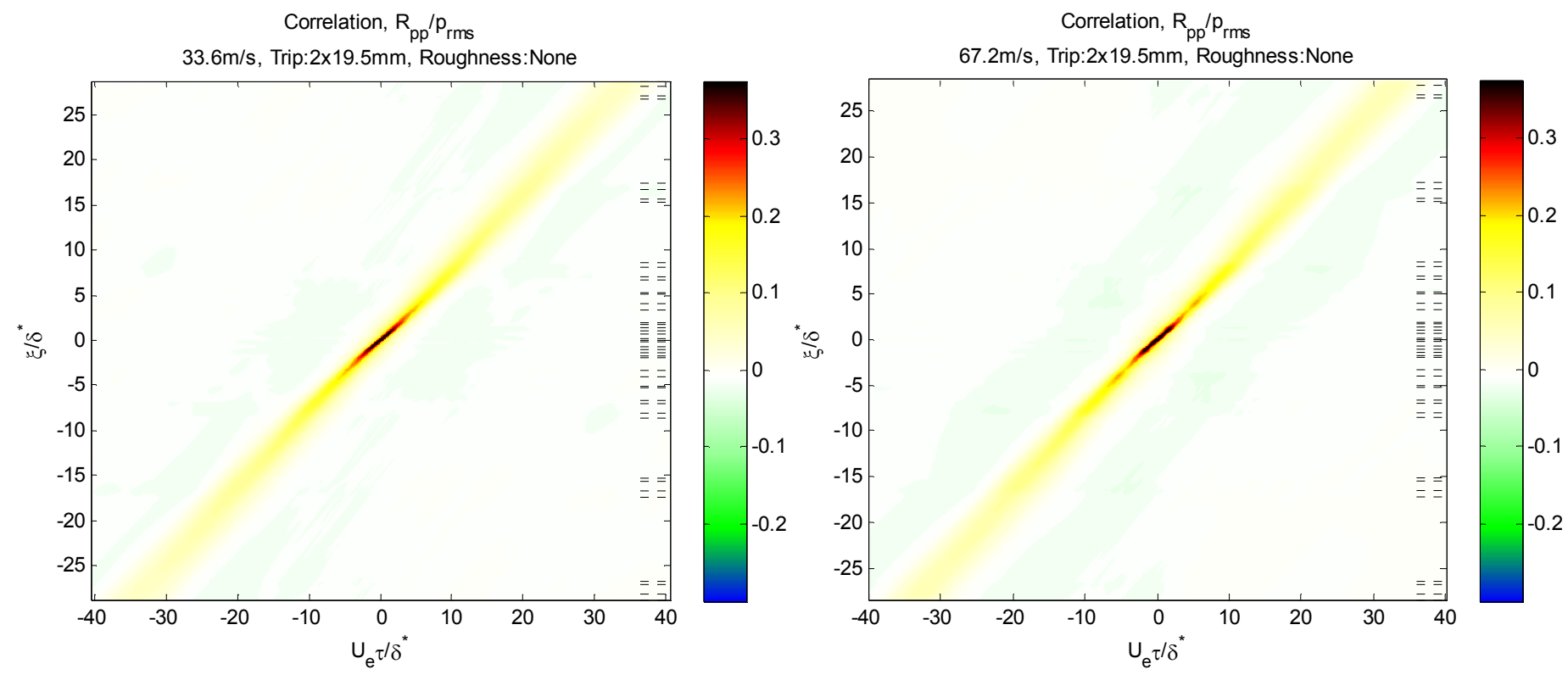

Figure 3.29. Smooth wall pressure spectra correlation for streamwise separations.
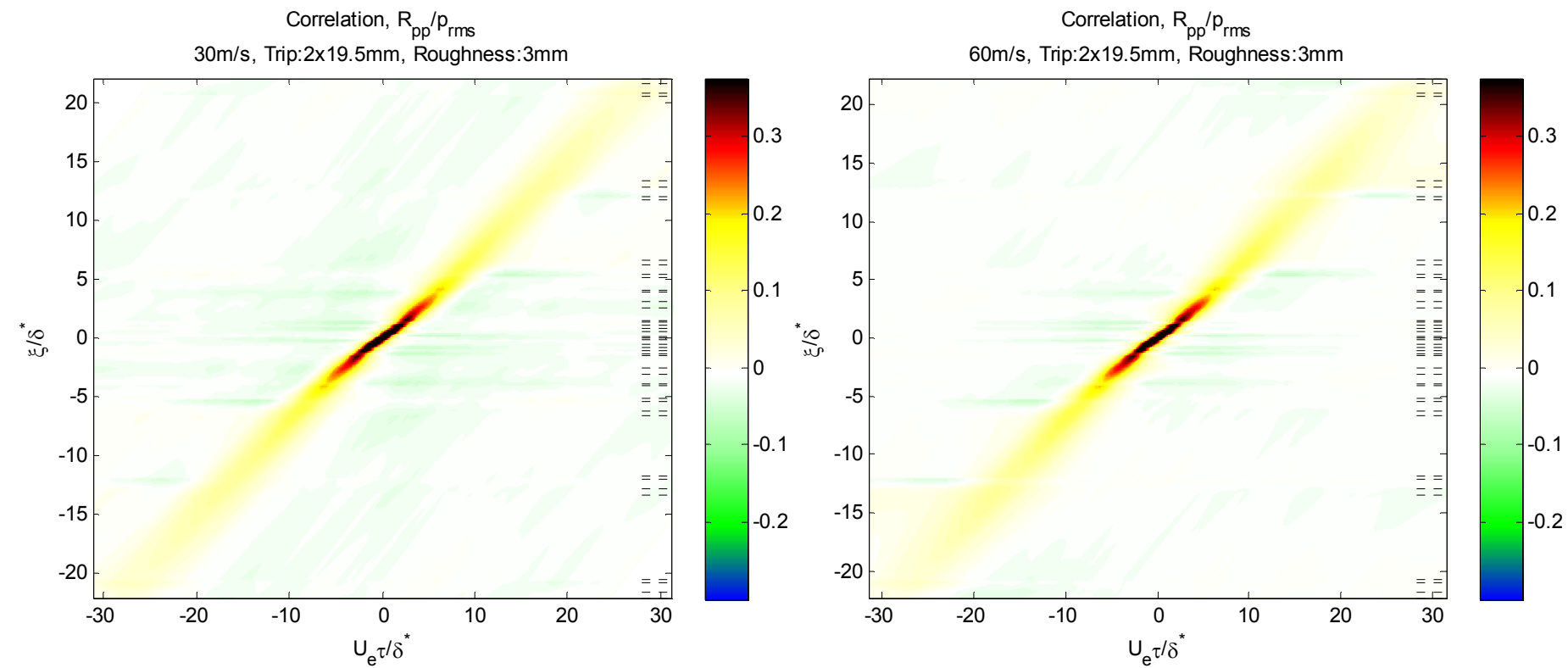

Figure 3.30. Rough wall pressure spectra correlation for streamwise separations. 


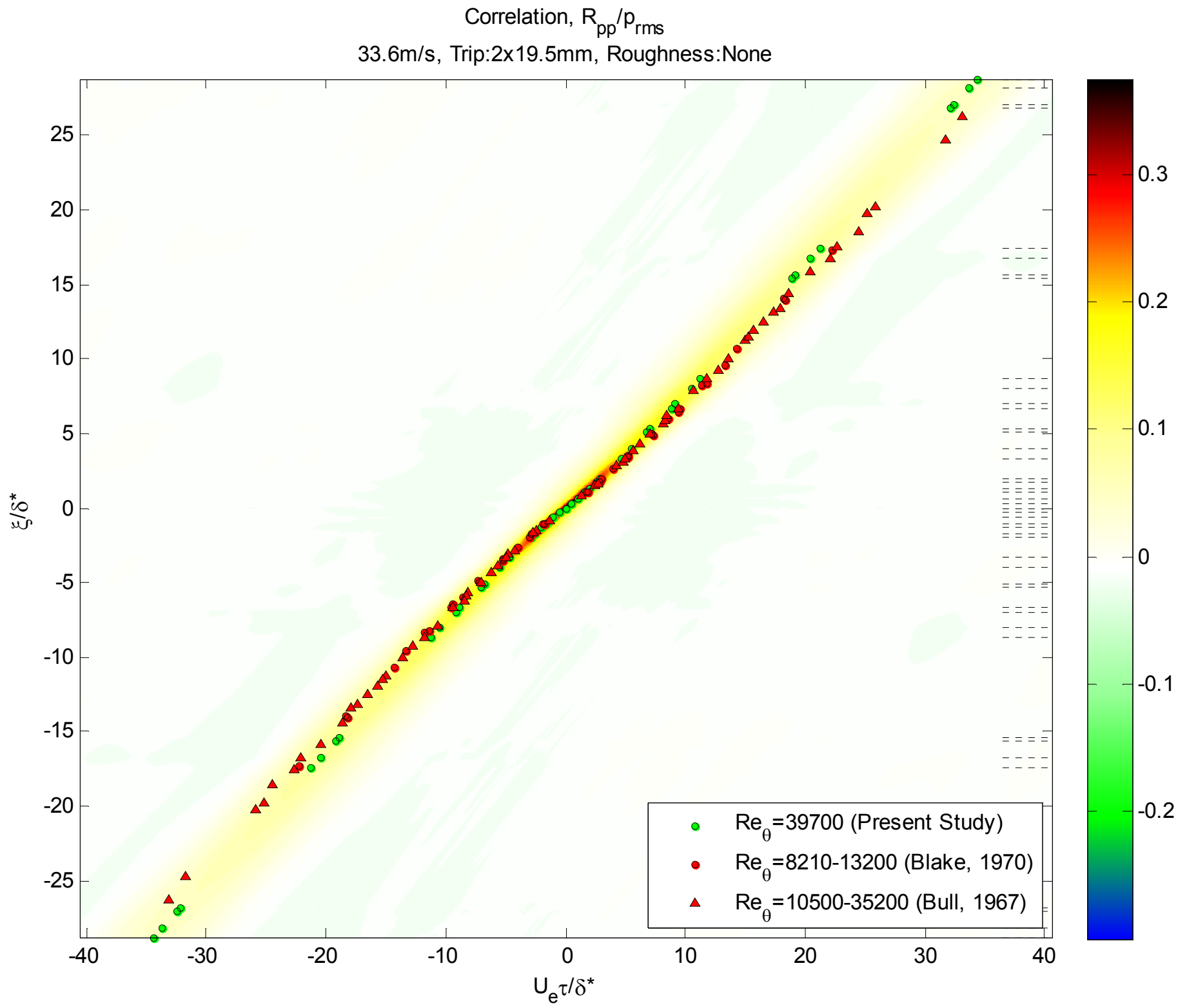

Figure 3.31. Smooth wall pressure spectra correlation and convective ridge for streamwise separations at 33.6-m/s. 
Correlation, $\mathrm{R}_{\mathrm{pp}} / \mathrm{p}_{\mathrm{rms}}$

30m/s, Trip:2x19.5mm, Roughness:3mm

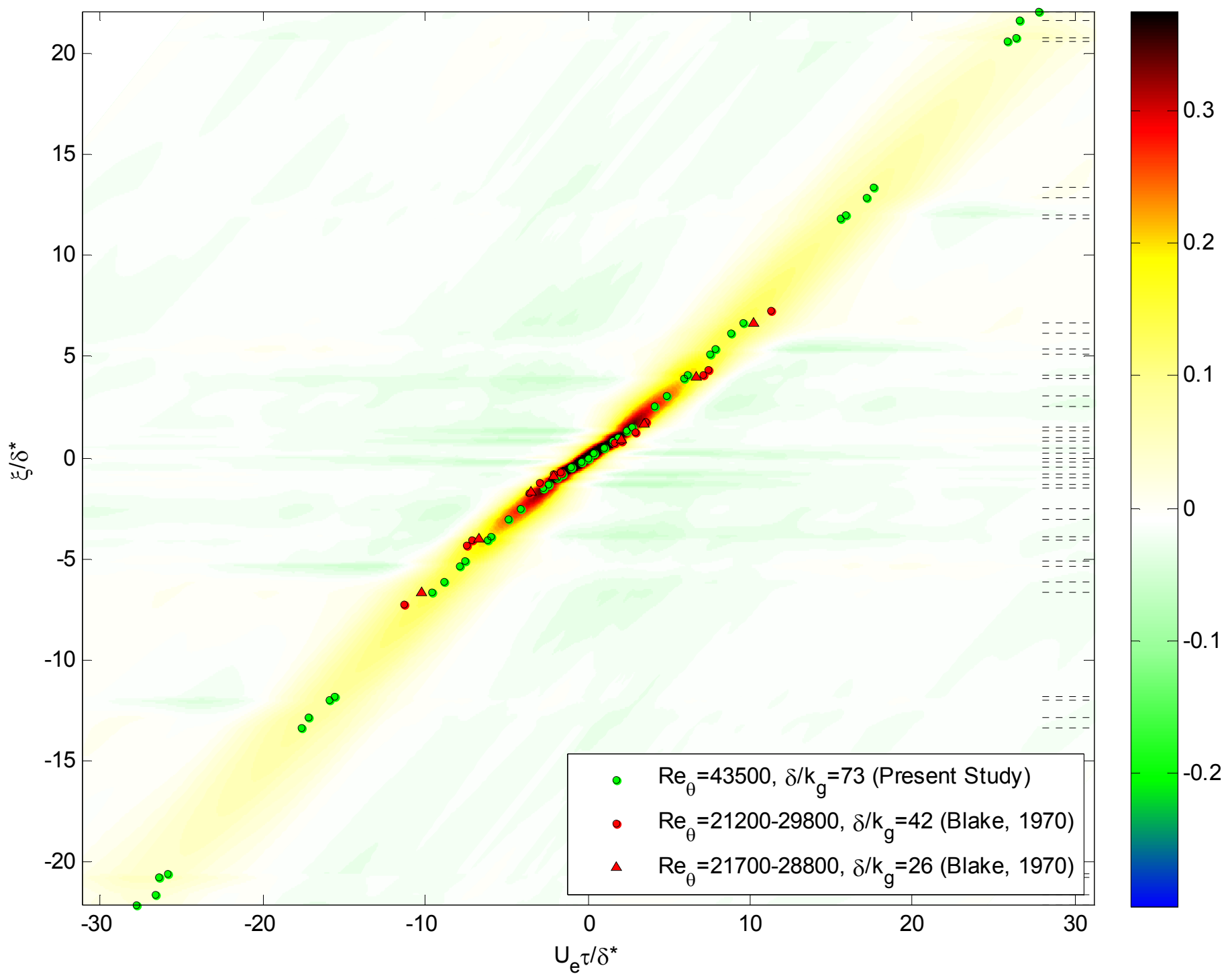

Figure 3.32. Rough wall pressure spectra correlation and convective ridge for streamwise separations at 30$\mathrm{m} / \mathrm{s}$.

After acoustic contamination was removed as described in section 2.6.1, spanwise separations were found to be largely uncorrelated for both smooth and rough walls when compared to streamwise separations as seen in Figure 3.33 and Figure 3.34. There was some small wall pressure correlation $\left(R_{p p} / p_{R M S} \geq 0.05\right)$ found at spanwise separations out to $3.3 \delta^{*}$ and $1.7 \delta^{*}$ along the smooth and rough walls respectively, though these are believed to be due to occasional single large eddies passing by and influencing the wall pressures up to these distances. This reasoning is supported by the fact that the spanwise space-time correlation seems to not be a function of time, with all positive correlations lying near the zero time delay axis $\tau=0$. 


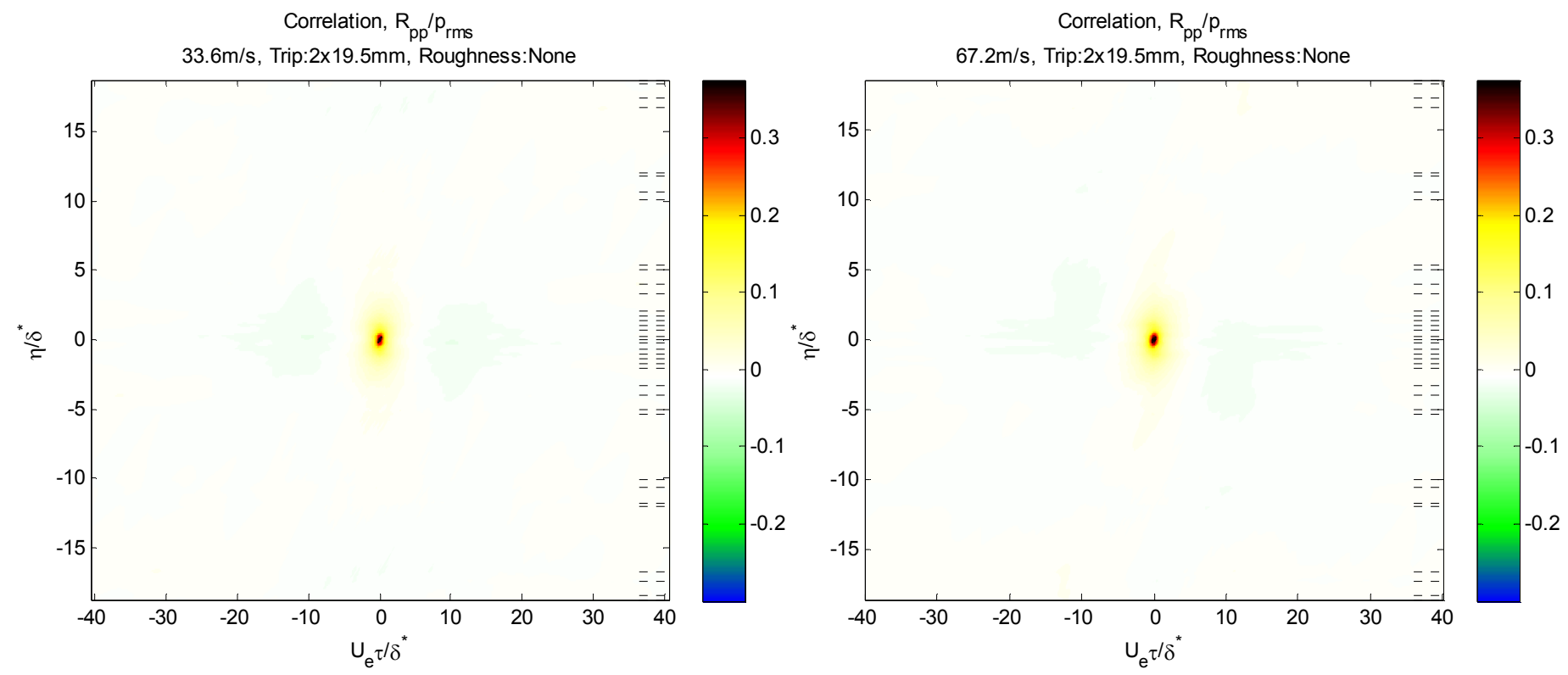

Figure 3.33. Smooth wall pressure spectra correlation for spanwise separations.
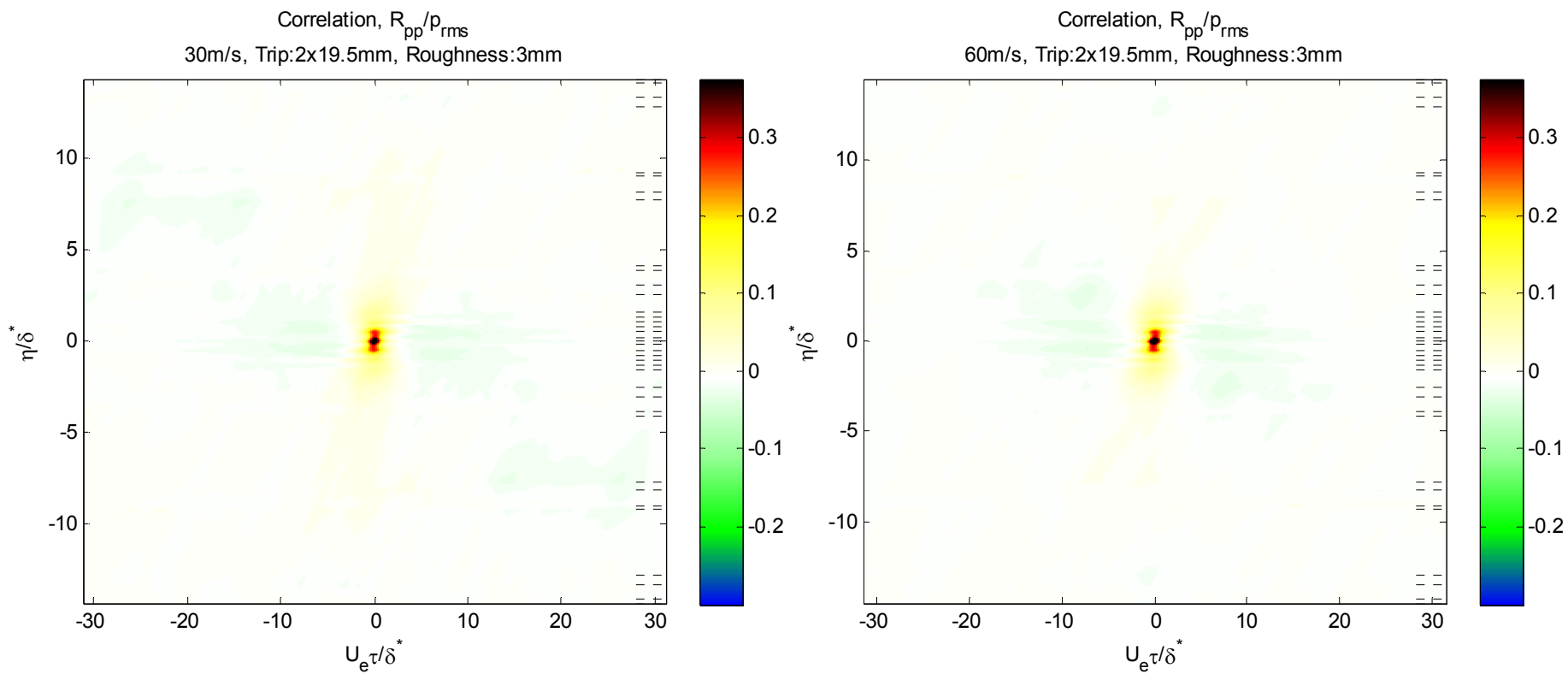

Figure 3.34. Rough wall pressure spectra correlation for spanwise separations.

Figure 3.35 and Figure 3.36 show the decay of the wall pressure space-time correlations along fixed streamwise separation distances for smooth and rough wall flows. The space-time correlation has been normalized about the root mean square (R.M.S.) pressure and is shown as a function of the time delay $\tau$ normalized about edge velocity $U_{e}$ and boundary layer displacement thickness $\delta^{*}$ for various streamwise separations $\xi / \delta^{*}$. The wall pressure correlations for all speeds $(22.4,33.6,44.8,56.0$ and $67.2-\mathrm{m} / \mathrm{s}$ for smooth wall and 20,30, 40, 50 and 60-m/s for rough wall) have been combined on the respective smooth and rough wall plots with matching separation distances grouped together. Boundary layer displacement thickness did change by $<5.5 \%$ for each flow speed, though 
the associated $\xi / \delta^{*}$ values have been grouped together for comparison with the average $\xi / \delta^{*}$ between the flow speeds being shown in the legend.

The smooth wall pressure space-time correlation decay shown in Figure 3.35 clearly illustrates how the convection pattern is not constant. In this figure it can be seen that as separation distances increase, the correlation is found to broaden and see decreased peak values. This would correspond with the notion that the turbulent structures are dispersed throughout the boundary layer and thus convected at varying speeds. When compared to the findings of Blake (1970), the present study is shown to see higher correlation values at larger separations possibly due to increased instrument resolution found in the present study. When comparing the space-time correlation decay envelopes from the different studies, one can see the different results in terms of the rate at which the pressure spectra decay over time. Integrating this curve with respect to time delay $\tau$ yields the Lagrangian timescale $T_{L}$. For the present data set, this was found to be near $T_{L}=5.8 \delta^{*} / U_{e}$, while Lagrangian timescale was found to be only $T_{L}=5.1 \delta^{*} / U_{e}$ for Blake's data set, due mostly to its more rapid correlation decay at large separation values.

Figure 3.36 shows the rough wall space-time correlation decay. Similar to the smooth wall data, the correlation is shown to broaden and see decreased peak values with increasing separation distances. However, the smooth wall data is also shown to decay at a faster rate than what was found over the smooth wall, indicative of the interference caused by the roughness elements as they promote increased mixing and decorrelation of the turbulent structures inside the boundary layer. Indicative of this increased decay is the shorter Lagrangian timescale of about $T_{L}=4.6 \delta^{*} / U_{e}$ found for the rough wall flows. Note that when compared to Blake's rough wall flows, the correlation decay was again found to be slower in the present data at larger separations. However, the present study is also shown to have higher peak correlations even at small separations. This is believed to be a function of the larger relative boundary layer to roughness height ratio $\left(\delta / \mathrm{kg}_{g}=72-76\right)$ found in this study as compared to the S-S $\left(\delta / k_{g}=42\right)$ and D-L $\left(\delta / k_{g}=26\right)$ roughness investigated by Blake. 


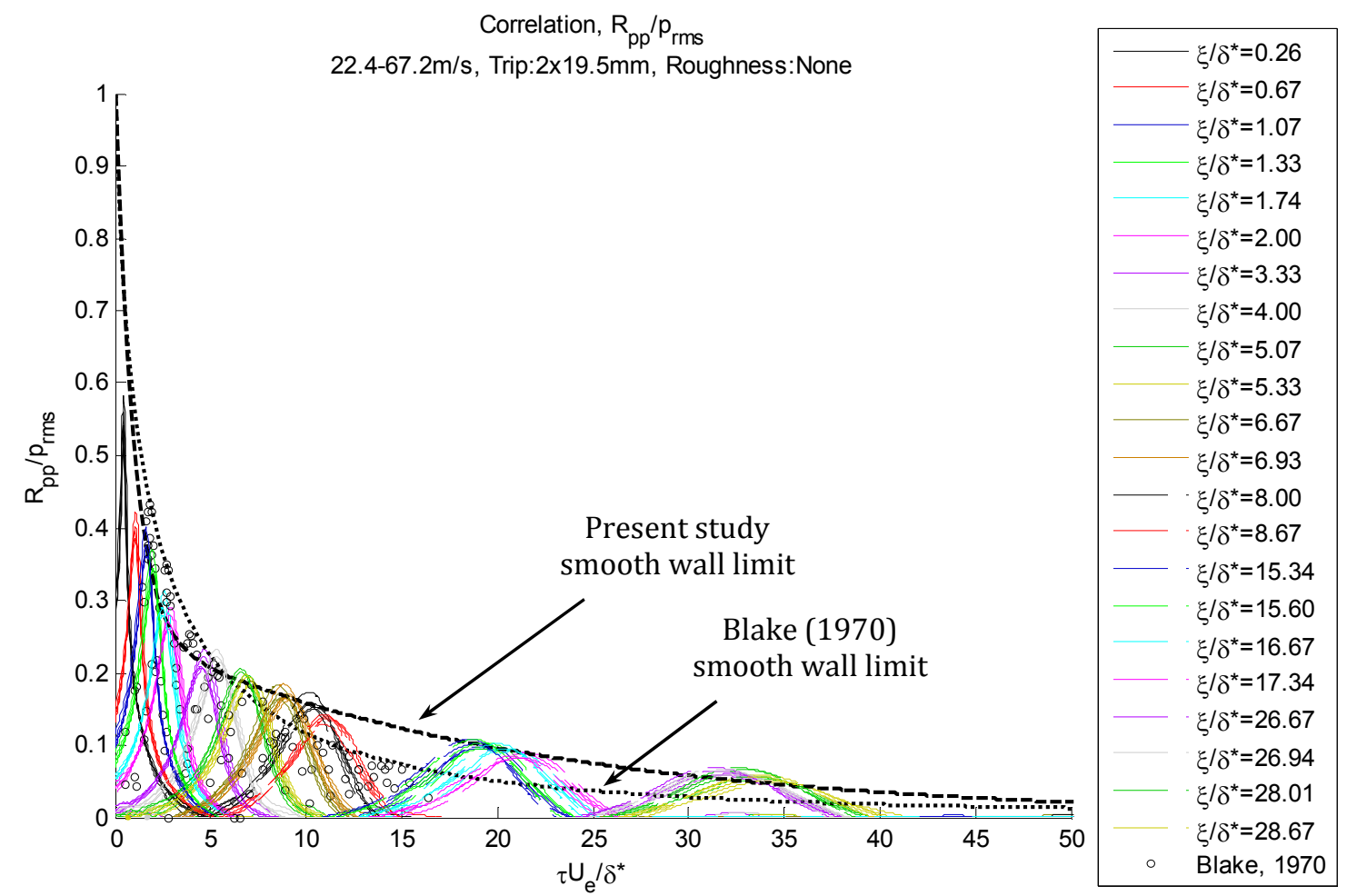

Figure 3.35. Smooth wall pressure spectra correlation decay for streamwise separations at 22.4, 33.6, 44.8, 56.0 and $67.2-\mathrm{m} / \mathrm{s}$.

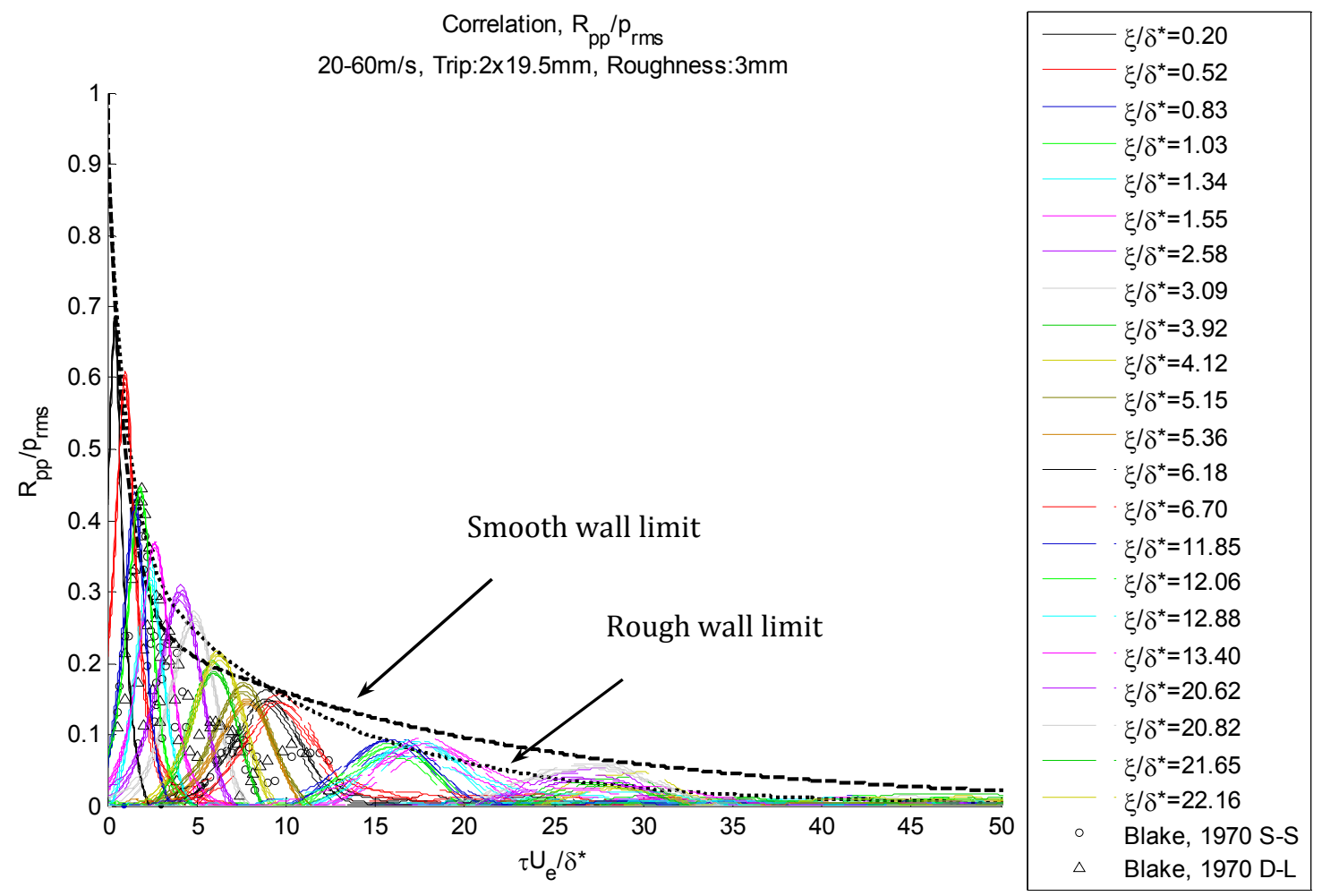

Figure 3.36. Rough wall pressure spectra correlation decay for streamwise separations at 20,30, 40, 50 and $60-\mathrm{m} / \mathrm{s}$. 


\subsubsection{Phase, Phase Convection Velocity and Convection Velocity}

The wall pressure spectra phase angle between distinct microphone locations was determined as the cross-spectral angle between measurement locations. Figure 3.37 and Figure 3.38 show the phase angles as a function of angular frequency $\omega$ normalized about boundary layer displacement thickness $\delta^{*}$ and edge velocity $U_{e}$ for streamwise separations, while Figure 3.39 and Figure 3.40 show the spanwise separation phase angles.

The smooth wall phase in Figure 3.37 shows increasing phase with increasing streamwise separation $\xi / \delta^{*}$, as well as the phase dependence on frequency along fixed separation lines. The frequency dependence was again known to be due to the segregation of the turbulent eddy structures by size throughout the boundary layer of gradient velocity. A coherence loss across all frequencies with increasing separations was previously observed in section 3.4.4, with high frequencies seeing the most rapid decline. When comparing the phase angle fixed-separation lines with one another, one sees a more rapid increase in phase with increasing frequency. These observations would suggest that for similarly sized turbulent eddies, those moving faster and tending towards the outer portions of the boundary layer would be the first to decorrelate, while similarly sized eddies moving slower would tend to be more stable.

Comparing the rough wall phase angle in Figure 3.38 to that of the smooth wall, one sees many of the same trends. The phase angle dependence on frequency as well as the increased phase slope due to increasing separation distances are both still present. The rough wall pressure spectra phase does, however, exhibit slightly negative phase angles at low frequencies for the smallest separation distance $0.20 \delta^{*}$. This feature is believed to be due to some sort of vortex shedding off of local roughness elements as it was not observed for separations larger than the element spacing $\left(\xi / \delta^{*} \cong 0.5\right)$.

The phase angles for spanwise separations $\eta$ between $0.2 \delta^{*}$ and $22.3 \delta^{*}$ for smooth and rough walls are shown in Figure 3.39 and Figure 3.40. Large phase angle are not present in these figures as would be expected in the absence of a spanwise convective mechanism for the turbulent eddies. As was stated in previous sections, it could be argued that over the smooth wall, phase angles between any two points would largely be due to turbulent eddy size, allowing a turbulent structure to simultaneously affect multiple spanwise locations. This assertion is somewhat supported by only small separation lines which are represented at higher frequencies, with essentially no phase data or meaningful coherence (section 3.4.4) present for separations larger than $5.09 \delta^{*}$. The rough wall data shows a similar behavior, though with slightly elevated phase values at low frequencies. It is believed that this is due to the more chaotic nature of the rough wall flow, as the increased boundary layer mixing due to surface roughness could cause some spanwise turbulent eddy drift across different spanwise locations. 
Phase $\alpha$

33.6m/s, Trip:2x19.5mm, Roughness:None

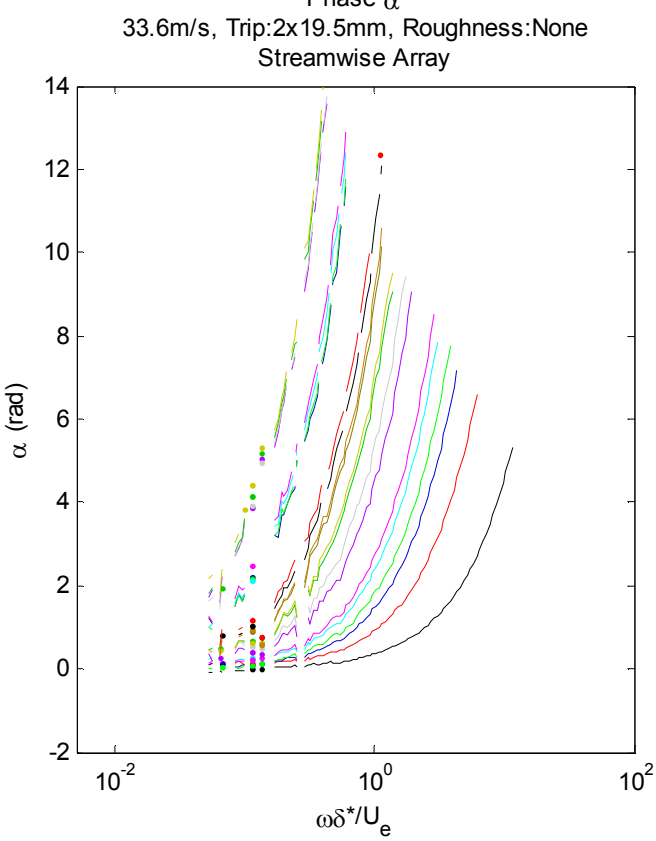

Phase $\alpha$

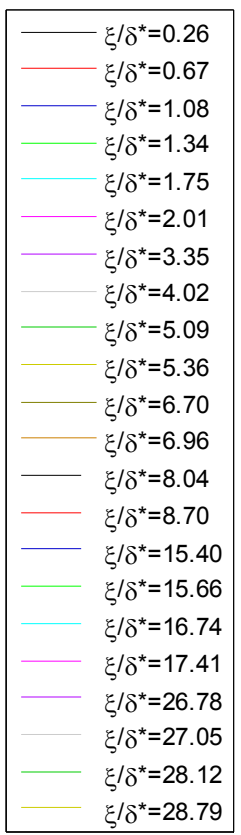

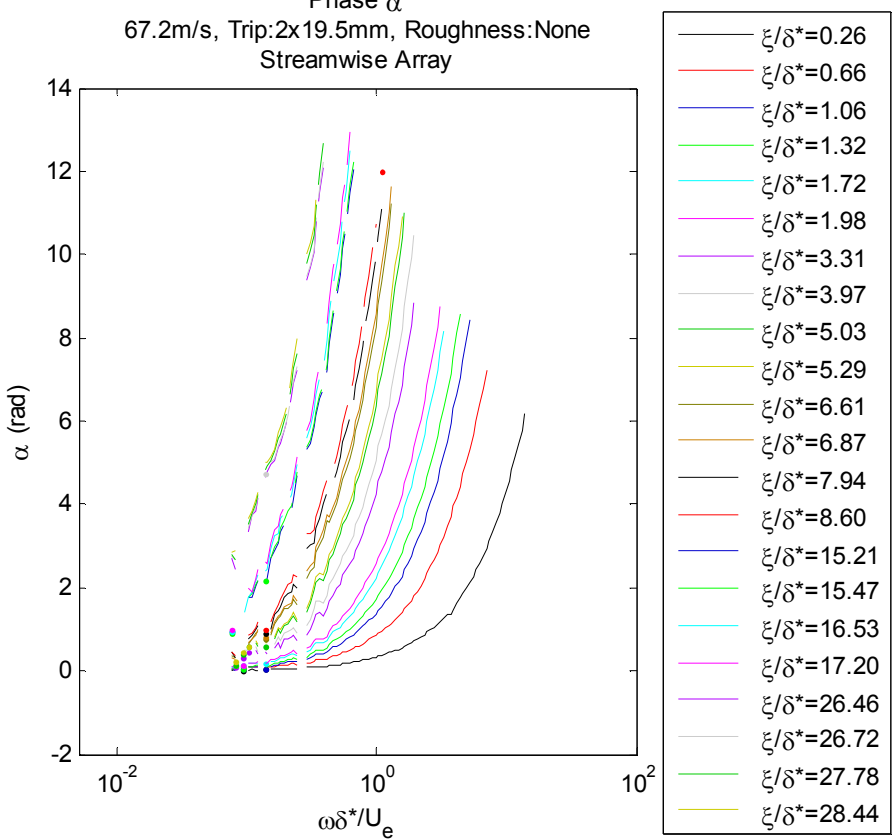

Figure 3.37. Smooth wall pressure spectra phase angle for streamwise separations.
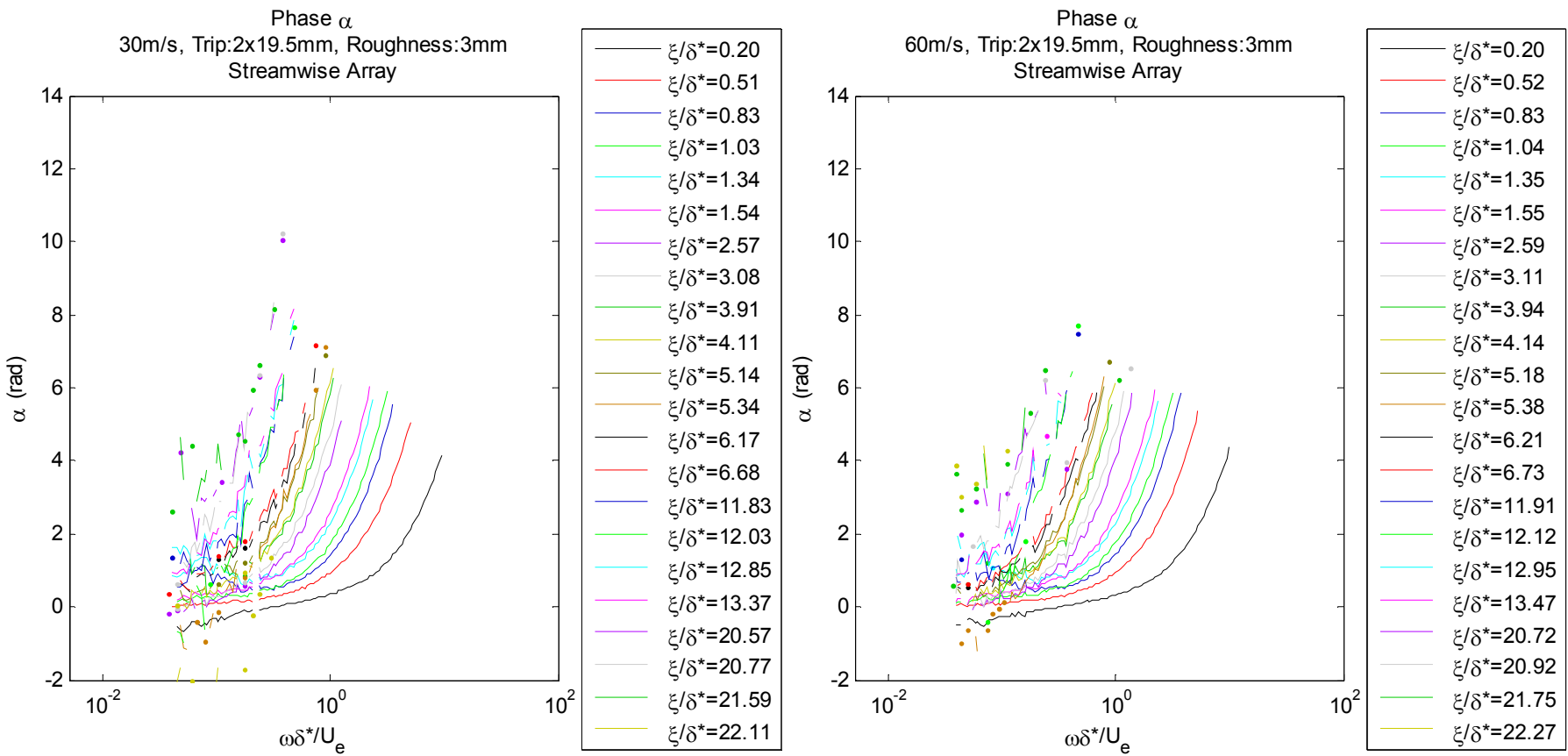

Figure 3.38. Rough wall pressure spectra phase angle for streamwise separations. 
Phase $\alpha$

33.6m/s, Trip:2x19.5mm, Roughness:None Spanwise Array

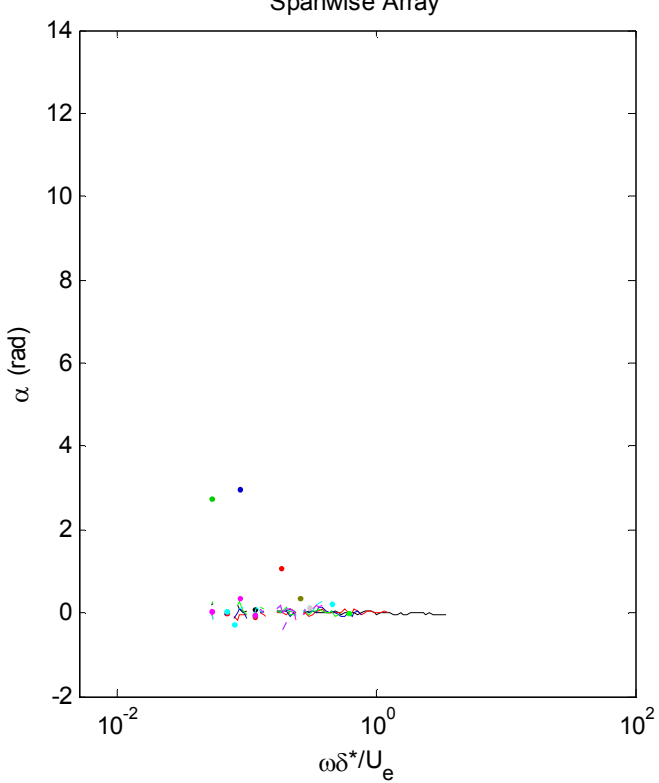

Phase $\alpha$

$67.2 \mathrm{~m} / \mathrm{s}$, Trip:2x19.5mm, Roughness:None Spanwise Array

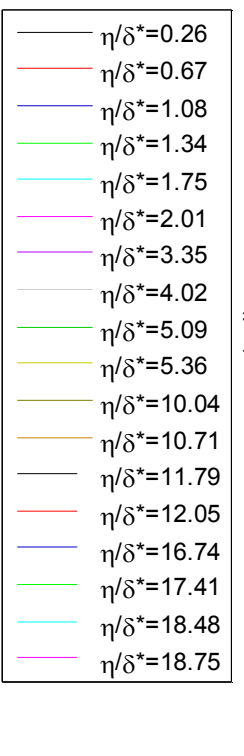

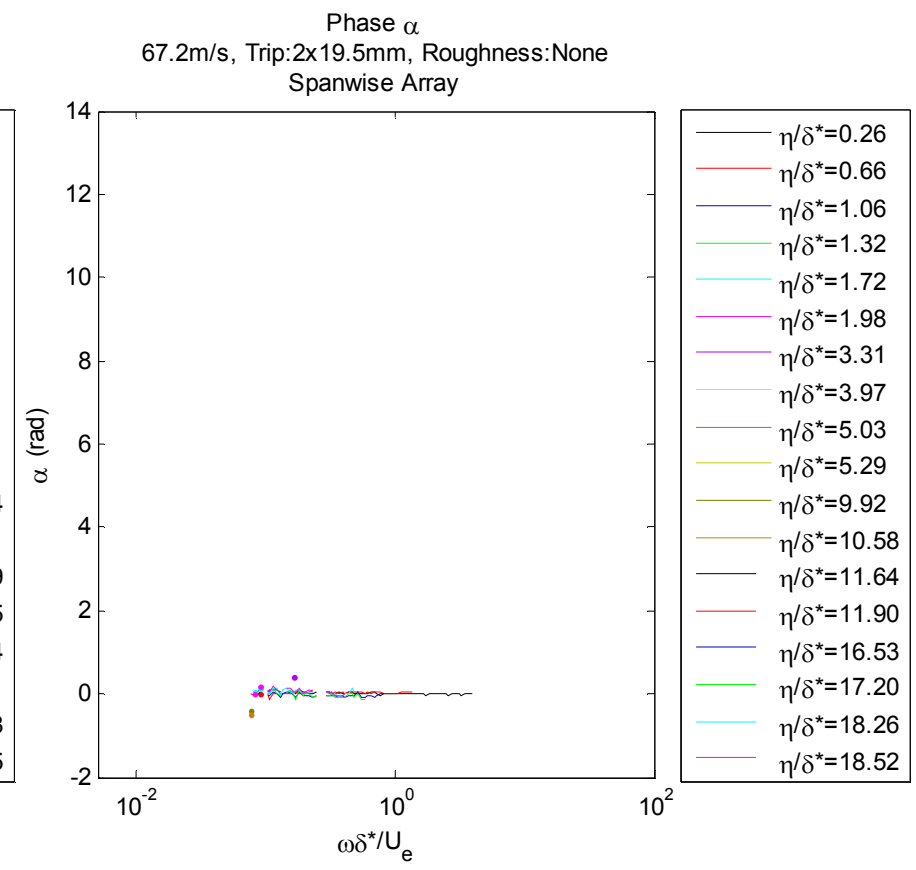

Figure 3.39. Smooth wall pressure spectra phase angle for spanwise separations.

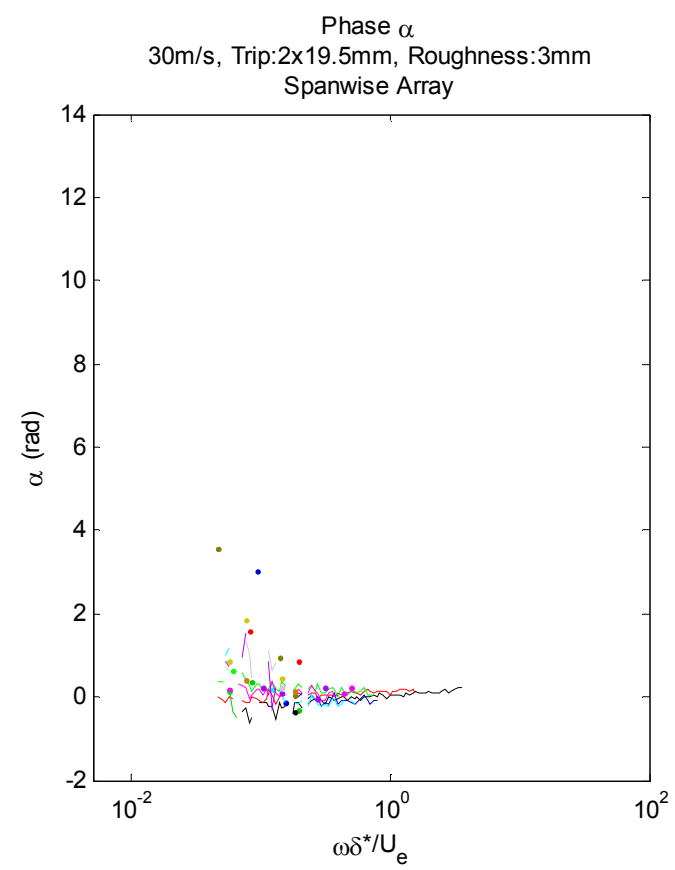

Phase $\alpha$

60m/s, Trip:2x19.5mm, Roughness:3mm Spanwise Array

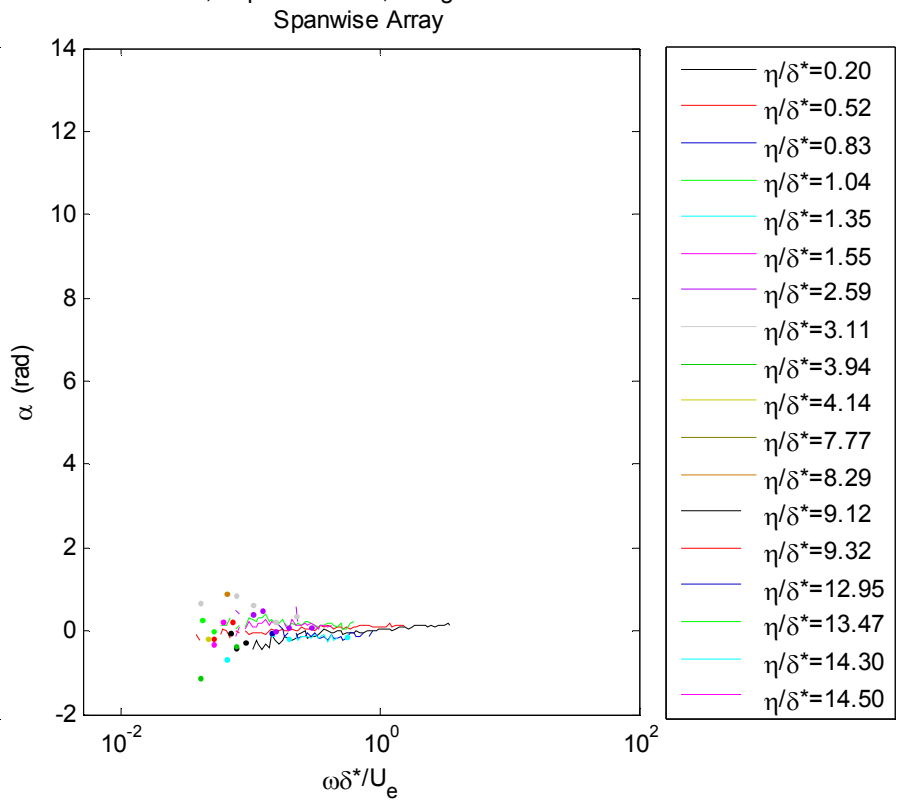

Figure 3.40. Rough wall pressure spectra phase angle for spanwise separations.

Following Blake (1970), the phase convection velocity $U_{c p}$ was found using Equation 3.20. In this equation, the wall convection velocity is the product of the separation distance $s$ and angular frequency $\omega$ divided by the phase angle $\alpha$.

$$
U_{c p}(s, \omega)=\frac{s \omega}{\alpha(s, \omega)}
$$


In Figure 3.41, the phase convection velocities are plotted for flows over a smooth surface. In this figure, the frequency $\omega$ is normalized on displacement thickness $\delta^{*}$ and edge velocity $U_{e}$, while streamwise separation distances $\xi$ are normalized on boundary layer displacement thickness $\delta^{*}$ for smooth surfaces between 22.4 and $67.2-\mathrm{m} / \mathrm{s}$. It is shown that the phase convection velocities over smooth walls were found to be between $0.03 U_{e}-$ $0.08 U_{e}$ higher than results previously found by Blake (1970), though a similar behavior and shape in response to the normalized frequency was observed. It is believed that the differences in phase convection velocities are somewhat dependent on Reynolds number. This supposition is supported by the slow yet steady increase in phase convection velocities observed by the present data with flow conditions at $22.4-\mathrm{m} / \mathrm{s}, R e_{\theta}=27400$ and the $67.2-\mathrm{m} / \mathrm{s}, R e_{\theta}=76700$ flow. For comparison, the maximum momentum thickness based Reynolds number reached by Blake was $R_{\theta}=17000$ for smooth surfaces.

The data in Figure 3.41 shows the dependence of phase convection velocity on frequency with a discernible peak between $\omega \delta^{*} / U_{e}=0.1-0.4$. The peak of the maximum phase convection velocity is shown to shift backwards in the frequency domain and up in normalized phase convection velocity with an increase in streamwise separations $\xi / \delta^{*}$. Following the results of Morrison et al. (1992) and assuming the outer turbulent structures are not fully attached eddies and may have some drift in their boundary layer position, the shifting convection velocity peak is suggestive that the turbulent eddies forming in the outer portions of boundary layer are slowly moving away from the wall and being carried by the faster portions of the boundary layer as they progress downstream. It is interesting to note that even at the lowest separations, this peak would be located just outside the overlap region of the wall pressure spectral plots shown in section 3.4.2. 

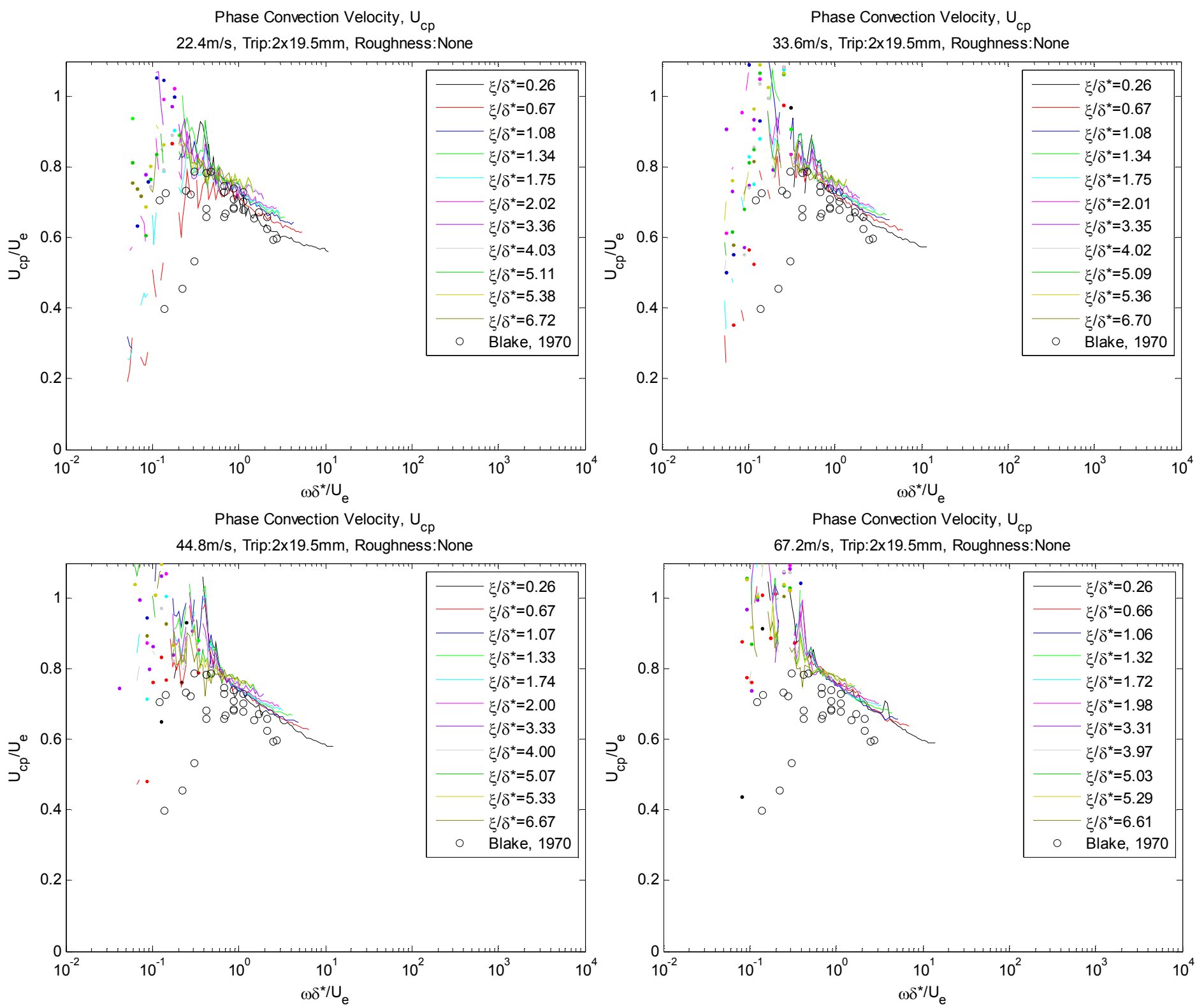

Figure 3.41. Smooth wall phase convection velocities.

Figure 3.42 shows the wall pressure spectra phase convection velocities over rough walls for flows between 20 and $60-\mathrm{m} / \mathrm{s}$, along with the normalized boundary layer thickness to roughness height ratio $\delta / \mathrm{kg}_{\mathrm{g}}$ for each data set. The phase convection velocities in the present study were again found to be between $0.02 U_{e}-0.08 U_{e}$ higher than the results found by Blake (1970), similar to what was seen over smooth walls. The phase convection velocity's dependence on Reynolds number still appears for the rough wall flows, though the effects are noticeably decreased. For the rough wall flows, the maximum Reynolds numbers reached in Blake's study were $R e_{\theta}=29800$ for a $\delta / k_{g}=42$, and $R e_{\theta}=$ 28800 for $\delta / k_{g}=26$. By comparison, the present study saw a momentum thickness based Reynolds number of $R e_{\theta}=28400$ for a $\delta / k_{g}=72$ at $20-\mathrm{m} / \mathrm{s}$, and $R e_{\theta}=80200$ for a $\delta / k_{g}=76$ at $60-\mathrm{m} / \mathrm{s}$. The relative roughness height $\delta / \mathrm{kg}_{g}$ also appears to have an effect on the phase 
convection velocity amplitudes. For the present study at $20-\mathrm{m} / \mathrm{s}$, the Reynolds number $R_{\theta}$ $=28400$ is very comparable to both sets of Blake's rough wall data $\left(R e_{\theta}=29800\right.$ and $R e_{\theta}=$ 28800). However, one can see that with increasing $\delta / \mathrm{kg}_{\mathrm{g}}$ and thus decreasing relative roughness sizes, the phase convection velocities also increase more towards the smooth wall levels as would be expected.
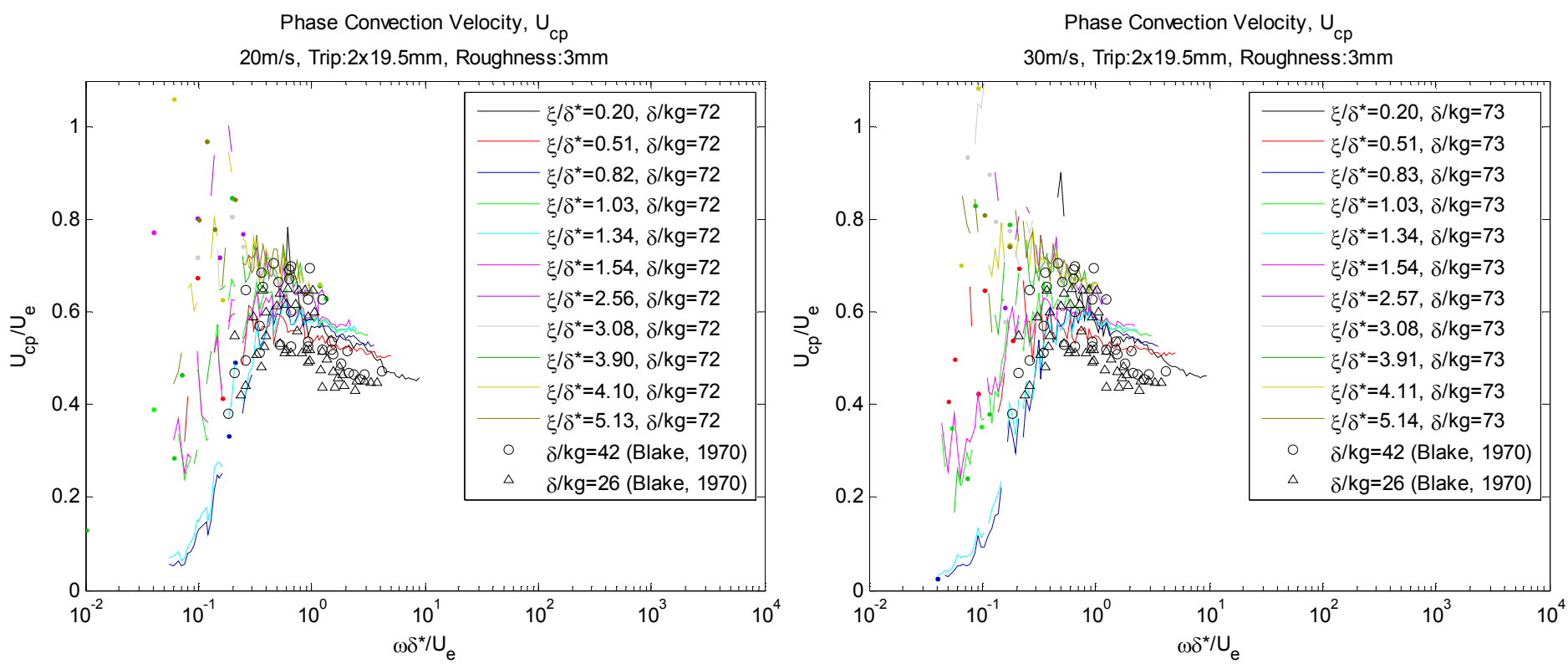

Phase Convection Velocity, $U_{c p}$
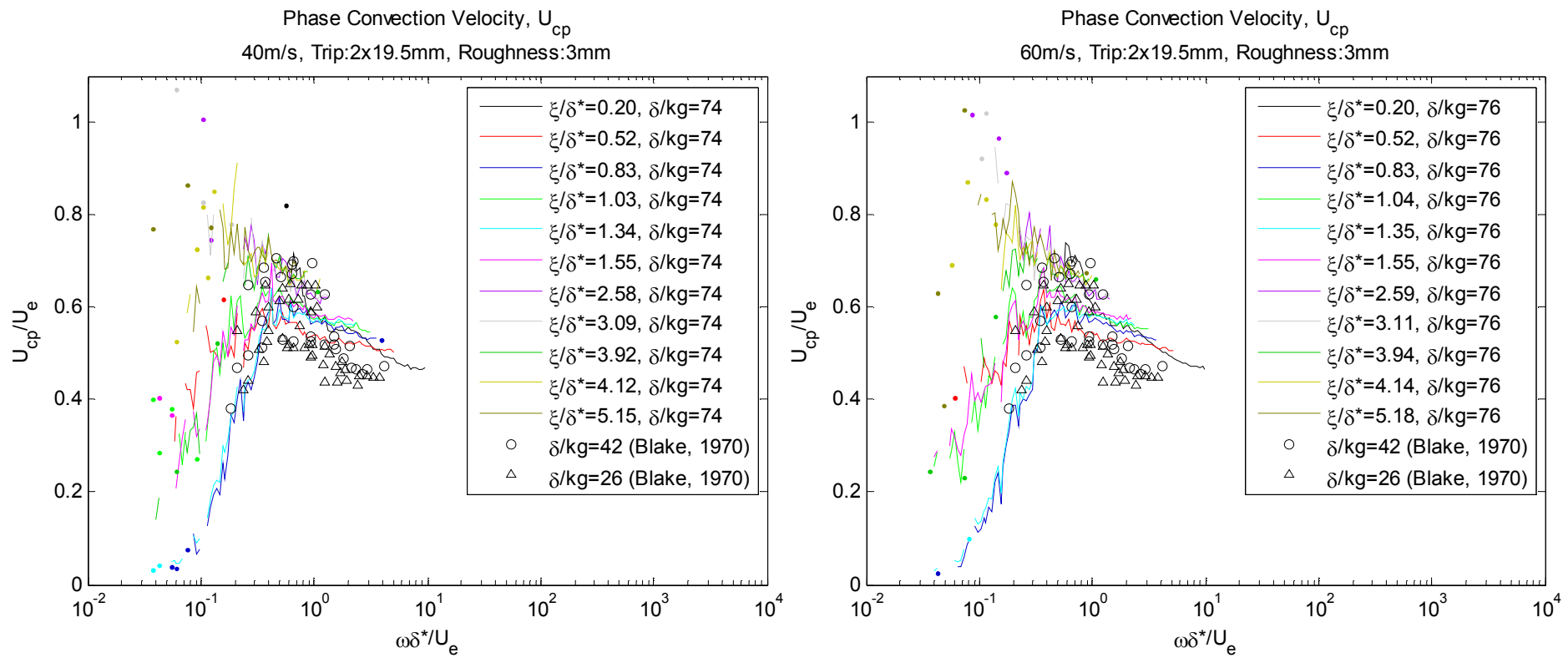

Figure 3.42. Rough wall phase convection velocities.

The phase convection velocity peak dependent on normalized streamwise separation distances $\xi / \delta^{*}$ found in the smooth wall conditions also appears over the rough walls in Figure 3.42. Over rough walls this peak appears to have shifted forward to be between $\omega \delta^{*} / U_{e}=0.2-1$, again showing a shift backwards in the frequency domain and up in normalized phase convection velocity with an increase in streamwise separations $\xi / \delta^{*}$. This 
suggests that the turbulent eddies forming in the boundary layer are still slowly moving away from the wall towards the outer, faster portions of the boundary layer, though the higher frequency shift reflects the delayed onset of the overlap region of the wall pressure spectral plots shown in section 3.4.3 due to the roughness turbulent kinetic energy shift towards the wall.

The wall pressure spectra convection velocity $U_{c}$ is defined as the slope of the convective ridge of the space-time correlation $R_{p p}$ and can be defined as the derivative of the separation distance with respect to the time delay as seen in Equation 3.21. In order to obtain this calculation, a regression line was fit along the convective ridge peaks (shown in Figure 3.31 and Figure 3.32) to be differentiated.

$$
U_{c}(s)=\frac{d s}{d \tau}
$$

The mean convection velocity could therefore be defined using Equation 3.22 as the separation distance $s$ instead divided by the time delay $\tau$. It should be noted that while providing somewhat more coarse results, not having to differentiate the data reduces the propagation of uncertainties when compared to the instantaneous convection velocity results.

$$
\overline{U_{c}}(s)=\frac{s}{\tau}
$$

The normalized convection velocities over smooth and rough surfaces for flow speeds between $22.4-67.4-\mathrm{m} / \mathrm{s}$ and $20-60-\mathrm{m} / \mathrm{s}$ respectively can be seen in Figure 3.43. The mean and instantaneous convection velocities $\overline{U_{c}}$ and $U_{c}$ are normalized about the edge velocity $U_{e}$, while the streamwise separation $\xi$ has been normalized about the displacement thickness $\delta^{*}$. In both smooth and rough wall cases, the mean convection velocity rapidly increases over short streamwise separations before leveling and approaching the asymptotic maximum of the instantaneous convection velocity.

When compared to the smooth wall results of Bull (1967) and Blake (1970) at the left of Figure 3.43, one notices the higher values of both mean and instantaneous convection velocities, with the previous studies reaching and asymptote of $0.83 U_{e}$ and the current study approaching $0.86 U_{e}-0.87 U_{e}$. While Reynolds number effects do not appear to be a contributing factor, it is unclear whether or not some uncertainty in the data is the cause for the convection velocity differences. As is shown in Figure 3.31, this moderate difference in the convection velocity is caused by only a minor difference in the space-time correlation convective ridge. While using a different method to obtain the convection velocities grouped by frequency, Farabee and Casarella also found convective velocities to approach $0.86 U_{e}$ for larger separations over smooth walls. Another feature found in the present study's instantaneous convection velocity not shown by Bull and Blake is a small bump between $2-3 \delta^{*}$. In these other data sets, a mean line was simply drawn through the combined convective ridges and differentiated to obtain the convection velocity, possibly suppressing this feature. On closer inspection it appears that the mean convection velocity data points from these other studies also seem to have a similar ridge as is suggested by the present data. 
The rough wall convection velocities in Figure 3.43 show the mean convection velocities approaching the instantaneous asymptote as before, though the roughness has decreased the convection velocities to between $0.78 U_{e}-0.84 U_{e}$. It is believed that the blockage ratio $\delta / k_{g}$ has a strong effect on the maximum convection velocity as the faster moving turbulent eddies associated with these larger length scales are typically located in the outer portions of the boundary layer. Jiménez (2004) and others have linked the blockage ratio directly to a wall roughness element's effect on the logarithmic and outer portions of a boundary layer. This would therefore suggest that larger values of $\delta / \mathrm{kg}$, representing a lesser amount of boundary layer blockage, would thus behave more similarly to smooth wall results, while data sets with lower $\delta / \mathrm{kg}_{g}$ would experience increased boundary layer blockage and see a drop in maximum convection velocities.

Blake concluded in his paper that the convection velocity defect should be proportional to the mean velocity defect due to roughness. While some evidence of this exists, the large variation in the present study's convection velocity results make any observations less conclusive, with a larger range of blockage ratio results being needed. The maximum convection velocity variations seen within the present data set, especially that of the $\operatorname{Re}_{\theta}=43500$ case, are instead believed to be due to experimental uncertainty, brought on by the widening of the convective ridge by the wall roughness as discussed in section 3.4.5.
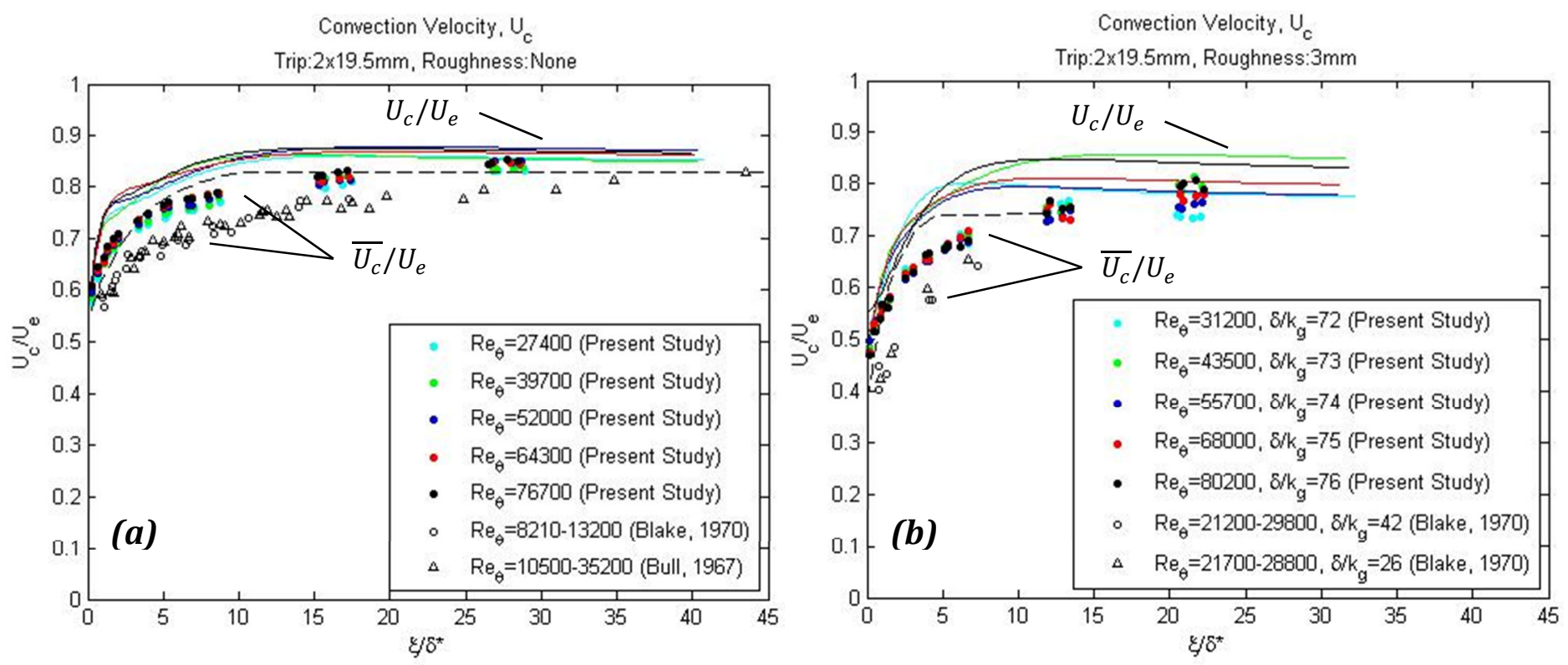

Figure 3.43. Convection velocities for (a) smooth and (b) rough walls. 


\section{CHAPTER 4. CONCLUSIONS}

The Virginia Tech Stability Wind Tunnel was used to measure the wall pressure fluctuations over a smooth and fully rough surface subjected to high Reynolds number turbulent boundary layer flows. Rough wall measurements utilized a rough surface composed of 3-mm hemispherical elements arranged in a $16.5-\mathrm{mm}$ square array. The wall pressure spectra was recorded using streamwise and spanwise microphone arrays for multiple flow conditions over both smooth and rough walls. Boundary layer velocity profiles and the mean pressure along the wall were also recorded. The conclusions drawn from this study follow.

\section{Smooth Surface Pressure Fluctuations}

- The inner variable scaling $\Phi(\omega) u_{t}^{2} /\left(\tau_{w}^{2} v\right) v s . \omega v / u_{t}^{2}$ was found to collapse the high Reynolds number smooth wall pressure spectra along both the overlap and roll off regions.

- Both classical $\left(\Phi(\omega) u_{t} /\left(\tau_{w}^{2} \delta\right) v s . \omega \delta / u_{t}\right)$ and mixed $\left(\Phi(\omega) U_{e} /\left(\tau_{w}^{2} \delta^{*}\right) v s . \omega \delta^{*} / U_{e}\right)$ outer variable scaling collapsed the pressure spectra along the low frequency and overlap regions within the present study. Classical scaling, however, appeared to be more consistent in obtaining a full collapse with other smooth wall studies.

- The pressure spectra overlap region was found to have a frequency dependence of $\omega^{-0.83}$, consistent with several other experimental results, but inconsistent with the theorized dependence at high Reynolds numbers. It was shown that some of the assumptions made in theorizing an $\omega^{-1}$ dependence may be flawed.

- The maximum coherence of the pressure spectra was generally found to decay following the form $\Gamma(\omega, \xi)=e^{-C_{1}\left|\omega \xi / U_{c p}\right|}$, where the constant $C_{1}$ was found to have a flow velocity dependence.

- The wall pressure convection velocity was found to asymptotically approach $0.86 U_{e}$ at large separations.

\section{Rough Surface Pressure Fluctuations}

- The roughness based inner variable pressure spectra scaling proposed by Blake in $1970\left(\Phi(\omega) u_{t} /\left(\tau_{w}^{2} k_{g}\right) v s . \omega k_{g} / u_{t}\right)$ was found to exhibit a Reynolds number dependence in the high frequency roll off region for the present data. A poor collapse was also shown between the different roughness configurations from a range of studies.

- Varano's (2010) proposed fully rough, inner variable pressure spectra scaling $\Phi(\omega) /\left(\rho^{2} U_{e}^{3} k_{g}\right) v s . \omega k_{g} / U_{e}$ performed similarly to Blake's (1970) and failed to collapse the high frequency roll off region of the rough wall spectra. 
- A new inner variable roughness based scaling was introduced as $\Phi(\omega) u_{t}^{1.5}$ / $\left(\tau_{w}^{2} v^{0.5} k_{s}^{0.5}\right) v s . \omega v^{0.5} k_{s}^{0.5} / u_{t}^{1.5}$ and shown to provide a collapse along the high frequency roll off region for a wide range of flows and roughness configurations.

- The proposed inner variable roughness based scaling suggests there are two timescales associated with the near wall turbulence structure generation: the timescale associated with the roughness height $k_{s} / u_{t}$, and the timescale associated with the wall bounded flow $v / u_{t}^{2}$.

- When applied to a range of hydraulically smooth, transitionally rough and fully rough flows, the proposed scaling collapsed the pressure spectra between a band defined at the upper frequency limit by the fully rough flows, and by the hydraulically smooth flows at the lower limit. Transitionally rough flows were contained between these limits thought to be influenced by the presence of the mean velocity laminar sublayer.

- An alternate inner variable roughness based scaling was proposed as $\Phi(\omega) u_{t}^{1.5}$ / $\left(\tau_{w}^{2} v^{0.5} k_{g}^{0.5}\right) v s . \omega v^{0.5} k_{g}^{0.5} / u_{t}^{1.5}$ which showed an improved high frequency collapse when compared to Blake's (1970) roughness based inner scaling, but not as universal when applied to other studies as the originally proposed scaling implementing $k_{s}$. This implied that the complete roughness configuration, not just the geometric height, influences the near wall turbulent structures.

- Both classical $\left(\Phi(\omega) u_{t} /\left(\tau_{w}^{2} \delta\right) v s . \omega \delta / u_{t}\right)$ and mixed $\left(\Phi(\omega) U_{e} /\left(\tau_{w}^{2} \delta^{*}\right) v s . \omega \delta^{*} / U_{e}\right)$ outer variable scaling collapsed the pressure spectra at low frequencies. Only mixed variable scaling was shown to provide some measure of collapse between smooth and rough wall spectra under similar flow conditions.

- A clearly defined overlap region was found at high Reynolds numbers for the rough wall pressure spectra. Evidence is presented that the frequency dependence of the rough wall overlap region is dependent on the roughness configuration and its transport of turbulent energy towards the wall.

- The maximum rough wall coherence of the pressure spectra was shown to decay following the same coherence function $\Gamma(\omega, \xi)=e^{-C_{1}\left|\omega \xi / U_{c p}\right|}$ as was used over smooth walls, though at an increased decay rate. This suggested the presence of roughness does not significantly disrupt the pressure decay mechanism other than increasing its rate.

- Surface roughness was found to broaden the convective ridge of the wall pressure space-time correlation, indicative of roughness interference of the convective velocities in the inner portions of the boundary layer.

- The rough wall pressure convection velocity was shown to approach between $0.78 U_{e}$ and $0.84 U_{e}$. There is evidence the blockage ratio $\delta / k_{g}$ due to roughness has a strong effect on the rough wall convection velocity. 


\section{REFERENCES}

Alexander, William Nathan. Normalization of Roughness Noise on the Near-Field Wall Pressure Spectrum. M.S. Thesis, Blacksburg, Virginia: Aerospace and Ocean Engineering Department, Virginia Tech, 2009.

Aupperle, F. A., and R. F. Lambert. "Effects of Roughness on Measured Wall-Pressure Fluctuations beneath a Turbulent Boundary Layer." The Journal of the Acoustical Society of America, 1970: Vol. 47, pp. 359-370.

Blake, William K. "Turbulent Boundary-Layer Wall-Pressure Fluctuations on Smooth and Rough Walls." Journal of Fluid Mechanics, 1970: Vol. 44, pp. 637-660.

Blake, William. "Turbulent Velocity and Pressure Fields in Boundary-Layer Flows Over Rough Surfaces." Turbulence in Liquids Symposium. University of Missouri-Rolla, October 5, 1971.

Borgoltz, Aurelien. Modifications of COherent Structures in Fan Blade Wakes for Broadband Noise Reduction. Ph.D Dissertation, Blacksburg, VA: Aerospace and Ocean Engineering Department, Virginia Tech, 2007.

Bradshaw, P. "'Inactive' Motion and Pressure Fluctuations in Turbulent Boundary Layers." Journal of Fluid Mechanics, 1967: Vol. 30, pp. 241-258.

Brzek, Brian, Sheilla Torres-Nieves, José Lebrón, Raúl Cal, Charles Meneveau, and Luciano Castillo. "Effects of Free-stream Turbulence on Rough Surface Turbulent Boundary Layers." Journal of Fluid Mechanics, 2009: Vol. 635, pp. 207-243.

Bull, M. K. "Wall-Pressure Fluctuations Associated with Subsonic Turbulent Boundary Layer Flow." Journal of Fluid Mechanics, 1967: Vol. 28, pp. 719-754.

Coles, Donald. "The Law of the Wake in the Turbulent Boundary Layer." Journal of Fluid Mechanics, 1956: Vol. 1, Iss. 2, pp. 191-226.

Corcos, G. M. "Resolution of Turbulence in Pressure." The Journal of the Acoustical Society of America, 1963: Vol. 35, No. 2, pp. 192-199.

Crede, Erin Dawne. Aerodynamics and Acoustics of the Virginia Tech Stability Tunnel Anechoic System. M.S. Thesis, Blacksburg, VA: Aerospace and Ocean Engineering Department, Virginia Tech, 2008.

Devenport, W. J., R. Burdisso, A. Borgoltz, R. Patricio, and M. Barone. "Aerodynamic and Acoustic Corrections for a Kevlar-Walled Anechoic Wind Tunnel." 16th AIAA/CEAS Aeroacoustics Conference. Stockholm, Sweden, June 7-9, 2010. AIAA-2010-3749.

Devenport, William J., Dustin L. Grissom, W. Nathan. Alexander, Benjamin S. Smith, and Stewart A.L. Glegg. "Measurements of Roughness Noise." Journal of Sound and Vibration, 2011: doi:10.1016/j.jsv.2011.03.017.

Dirling, R. B., Jr. "A Method for Computing Roughwall Heat Transfer Rates on Reentry Nosetips." 8th AIAA Thermophysics Conference. Palm Springs, California, July 16-18, 1973. AIAA-1973-763.

Farabee, Theodore M., and Mario J. Casarella. "Spectral Features of Wall Pressure Fluctuations beneath Turbulent Boundary Layers." Physics of Fluids, 1991: Vol. A3, No. 10, pp. 2410-2420.

Fernholz, H., and P. Finley. "The Incompressible Zero-Pressure Gradient Turbulent Boundary Layer: An Assessment of the Data." Progress in Aerospace Science, 1996: Vol. 32, pp. 245-311. 
Gloerfelt, Xavier. "The Link Between Wall Pressure Spectra and Radiated Sound From Turbulent Boundary Layers." 16th AIAA/CEAS Aeroacoustics Conference. Stockholm, Sweden, June 7-9, 2010. AIAA-2010-3904.

Goody, Michael. An Experimental Investigation of Pressure Fluctuations in ThreeDimensional Turbulent Boundary Layers. Ph.D Dissertation, Blacksburg, VA: Aerospace and Ocean Engineering Department, Virginia Tech, 1999.

Goody, Michael. "Emperical Spectral Model of Surface Pressure Fluctuations." AIAA Journal, 2004: Vol. 24, No. 9.

Gravante, S. P., A. M. Naguib, C. E. Wark, and H. M. Nagib. "Characterization of the Pressure Fluctuations Under a Fully Developed Turbulent Boundary Layer." AIAA Journal, 1998: Vol. 36, No. 10, pp. 1808-1816.

Horne, Michael P. Physical and Computational Investigation of the Wall Pressure Fluctuations in a Channel Flow. NRL Memorandum Report 6628, Washington, DC: Laboratory for Computational Physics and Fluid Dynamics, 1990.

Jiménez, Javier. "Turbulent Flows over Rough Walls." Annual Review of Fluid Mechanics, 2004: Vol. 36 pp. 173-196.

Klewicki, J. C., P. J. A. Priyadarshana, and M. M. Metzger. "Statistical Structure of the Fluctuation Wall Pressure and its In-Plane Gradients at High Reynolds Number." Journal of Fluid Mechanics, 2008: Vol. 480, pp. 195-220.

Long, Robert R., and Tien-Chay Chen. "Experimental Evidence for the Existence of the 'Mesolayer' in Turbulent Systems." Journal of Fluid Mechanics, 1981: Vol. 105, pp. 19-59.

McGrath, B., and R. Simpson. Some Features of Surface Pressure Fluctuations in Turbulent Boundary Layers with Zero and Favorable Pressure Gradients. NASA Contract Rep. 4051, Blacksburg, Va: Aerospace and Ocean Engineering Department, Virginia Tech, 1987.

Morrison, J. F., C. S. Subramanian, and P. Bradshaw. "Bursts and the Law of the Wall in Turbulent Boundary Layers." Journal of Fluid Mechanics, 1992: Vol. 241, pp. 75-108.

Morrison, Jonathan F. "The Interaction Between Inner and Outer Regions of Turbulent Wall-Bounded Flow." Philosophical Transactions of the Royal Society, 2007: Vol. 365, pp. 683-698.

Morton, Michael, and William J. Devenport. Understanding the Inflow to a Rotor Embedded in a Turbulent Boundary Layer. Yearly Progress Report, Contract N00014-10-0908, Blacksburg, Va: Aerospace and Ocean Engineering Department, Virginia Tech, 2011.

Österlund, J., B. Lindgren, and A. Johansson. "Flow Structure in Zero Pressure-Gradient Turbulent Boundary Layers at High Reynolds Numbers." European Journal of Mechanics B/Fluids, 2003: Vol. 22, pp. 379-390.

Panton, Ronald L., and John H. Linebarger. "Wall Pressure Spectra Calculations for Equilibrium Boundary Layers." Journal of Fluid Mechanics, 1974: Vol. 65 pt. 2, pp. 261-287.

Remillieux, M. C., et al. "Calibration and Demonstration of the New Virginia Tech Anechoic Wind Tunnel." 14th AIAA/CEAS Aeroacoustics Conference. Vancouver, British Columbia, May 5-7, 2008. AIAA-2008-2911.

Schetz, Joseph. Boundary Layer Analysis. Upper Saddle River, NJ: Prentice-Hall Inc., 1993. 
Schewe, Günter. "On the Structure and Resolution of Wall-Pressure Fluctuations Associated with Turbulent Boundary Layer Flow." Journal of Fluid Mechanics, 1983: Vol. 134, pp. 311-328.

Schlichting, Herman. Boundary-Layer Theory. McGraw-Hill, Inc, 1979.

Smith, B. S., H. E. Camargo, R. A. Burdisso, and W. J. Devenport. "Development and Testing of a Novel Acoustic Wind Tunnel Concept." 11th AIAA/CEAS Aeroacoustics Conference. Monterey, CA, May 23-25, 2005. AIAA-2005-3053.

Smith, Benjamin S. Wall Jet Boundary Layer Flows Over Smooth and Rough Surfaces. Ph.D Dissertation, Blacksburg, VA: Aerospace and Ocean Engineering Department, Virginia Tech, 2008.

Varano, Nathaniel D. Fluid Dynamics and Surface Pressure Fluctuations of Turbulent Boundary Layers Over Sparse Roughness. Ph.D Dissertation, Blacksburg, VA: Aerospace and Ocean Engineering Department, Virginia Tech, 2010.

Wittmer, K. S., W. J. Devenport, and J. S. Zsoldos. "A Four Sensor Hot Wire Probe System for Three Component Velocity Measurements." Experiments in Fluids, 1998: Vol. 24, pp. 416-423.

Wosnik, Martin, Luciano Castillo, and William K. George. "A Theory for Turbulent Pipe and Channel Flows." Journal of Fluid Mechanics, 2000: Vol. 421, pp. 115-145. 